\title{
Index theory and groupoids for filtered manifolds
}

\author{
Dissertation \\ for the award of the degree \\ "Doctor rerum naturalium" \\ at the Georg-August-Universität Göttingen
}

within the doctoral programme "Mathematical Sciences"

of the Georg-August University School of Science (GAUSS)

submitted by

Eske Ewert

from Munich

Göttingen, September 3, 2020 



\section{Thesis advisory committee}

Prof. Dr. Ralf Meyer,

Mathematisches Institut, Georg-August-Universität Göttingen

Professor Ryszard Nest,

Department of Mathematical Sciences, University of Copenhagen

\section{Members of the examination board}

\section{Referees}

Prof. Dr. Thomas Schick,

Mathematisches Institut, Georg-August-Universität Göttingen

Prof. Dr. Elmar Schrohe,

Institut für Analysis, Leibniz Universität Hannover

Associate Professor Niels Martin Møller,

Department of Mathematical Sciences, University of Copenhagen

\section{Other members of the examination board}

Prof. Dr. Dorothea Bahns,

Mathematisches Institut, Georg-August-Universität Göttingen

Jun.-Prof. Dr. Madeleine Jotz Lean,

Mathematisches Institut, Georg-August-Universität Göttingen

Prof. Dr. Max Wardetzky,

Institut für Numerische und Angewandte Mathematik, Georg-August-Universität Göttingen

Date of the oral examination: October 26, 2020. 



\section{Preface}

\section{Acknowledgements}

I would like to express my gratitude to all the people that accompanied me during the time of my $\mathrm{PhD}$ studies and contributed to it in one way or another.

First of all, I thank my supervisor Prof. Ralf Meyer for his guidance and advice in the last years. I am thankful to him for taking the time to answer my questions and sharing his knowledge. His suggestions helped to improve the presentation of this thesis. I also thank Prof. Ryszard Nest for his supervision and many useful discussions. Often, they helped me to get a new perspective on things when I was stuck. Moreover, I am very thankful to him for the opportunity to spend a part of my $\mathrm{PhD}$ at the University of Copenhagen and his hospitality.

I am grateful to Prof. Thomas Schick for many interesting lectures and for many helpful questions and comments concerning my work.

I thank Prof. Elmar Schrohe for agreeing to be a referee for this thesis. Furthermore, I would like to thank him and his group in Hannover for their warm welcome.

I would like to express my gratitude to Prof. Niels Martin Møller for refereeing this thesis. I thank Prof. Dorothea Bahns, Jun.-Prof. Madeleine Jotz Lean and Prof. Max Wardetzky for becoming part of the thesis committee. Moreover, I would like to thank Prof. Dorothea Bahns for the interesting lecture cycle on Mathematical Physics.

I thank Dr. Véronique Fischer for discussions during her stay in Göttingen and Prof. Raphaël Ponge for recommendations concerning the literature.

I am also thankful to all members of the RTG 2491 "Fourier analysis and spectral theory". It is very inspiring to be part of this group and I am looking forward to participating more actively again after handing in this thesis. I thank all members of the mathematical institute in Göttingen for many illuminating lectures, talks and discussions, which made it a pleasure to study here.

I would like to thank my fellow PhD students Ana, Celso and Dev for many mathematical and non-mathematical conversations. Especially, I was happy to meet them regularly online during the lockdown. Many thanks also go to Miriam for her friendship, I miss being able to just knock on the door of her office. Thanks to Jarl for the help with the Danish abstract. I thank all other friends in Göttingen and Copenhagen, the last years would not have been the same without them.

Finally, I thank my parents Petra and Hans-Joachim for their love and always believing in me and supporting me on my path. I am thankful to my sister Iva for constantly being there for me. I am deeply grateful to Eike for always cheering me up and without whom this would not have been possible. 


\begin{abstract}
In this thesis, we propose to use generalized fixed point algebras as an approach to the pseudodifferential calculus on filtered manifolds.

A filtered manifold is a manifold $M$ with a filtration of its tangent bundle which is compatible with the Lie bracket. This filtration allows to define a new notion of order for the differential operators on $M$. As a result, the highest order part of a differential operator is a family of right-invariant model operators acting on certain nilpotent Lie groups. These groups form the bundle of osculating groups of the filtered manifold. The new order can be encoded by a dilation action of $\mathbb{R}_{>0}$ on this bundle.

The tangent groupoid $\mathbb{T}_{H} M$ of the filtered manifold $M$ describes the relation between the operators acting on $M$ and their model operators on the osculating groups. It is equipped with a "zoom" action of $\mathbb{R}_{>0}$ that extends the dilations. In this thesis, we build the generalized fixed point algebra for the zoom action on a certain ideal $\rrbracket$ in the groupoid $\mathrm{C}^{*}$-algebra of $\mathbb{T}_{H} M$. This generalized fixed point algebra $\operatorname{Fix}^{\mathbb{R}_{>0}}(\mathbb{J})$ is a $\mathrm{C}^{*}$-subalgebra of the bounded operators on $L^{2}(M)$. Moreover, there is a "principal symbol map" $S_{H}$ which induces a short exact sequence
\end{abstract}

$$
\mathbb{K}\left(L^{2} M\right) \longleftrightarrow \operatorname{Fix}^{\mathbb{R}>0}(\mathbb{J}) \stackrel{S_{H}}{\longrightarrow} \operatorname{Fix}^{\mathbb{R}}>0\left(\mathbb{J}_{0}\right) .
$$

Here, the principal symbol map takes values in another generalized fixed point algebra $\operatorname{Fix}^{\mathbb{R}>0}\left(\mathbb{J}_{0}\right)$, where $\mathbb{J}_{0}$ is an ideal in the $\mathrm{C}^{*}$-algebra of the bundle of osculating groups. This symbol algebra is, in general, noncommutative. It is unital if $M$ is compact. In this case, call $P \in \mathrm{Fix}^{\mathbb{R}^{>0}}(\mathbb{J})$ elliptic if its principal symbol is invertible. We show that $P$ is elliptic if and only if all model operators satisfy the Rockland condition.

Furthermore, it is shown that the sequence above coincides with the $\mathrm{C}^{*}$-completion of the order zero pseudodifferential extension by van Erp and Yuncken vEY19. When viewing a graded Lie group as a filtered manifold, we show that the same holds for the calculus by Fischer, Ruzhansky and Fermanian-Kammerer developed in FR16 FFK17.

We prove that $\operatorname{Fix}^{\mathbb{R}>0}\left(\mathbb{J}_{0}\right)$ is KK-equivalent to the usual principal symbol algebra of functions on the cosphere bundle of $M$. Lastly, we present an index theorem, up to inverting the Connes-Thom isomorphism, for order zero pseudodifferential operators on a compact filtered manifold that are elliptic in this calculus.

In this thesis, we propose to use generalized fixed point algebras as an approach to the pseudodifferential calculus on filtered manifolds.

A filtered manifold is a manifold $M$ with a filtration of its tangent bundle which is compatible with the Lie bracket. This filtration allows to define a new notion of order for the differential operators on $M$. As a result, the highest order part of a differential operator is a family of right-invariant model operators acting on certain nilpotent Lie groups. These groups form the bundle of osculating groups of the filtered manifold. The new order can be encoded by a dilation action of $\mathbb{R}_{>0}$ on this bundle.

The tangent groupoid of a filtered manifold $M$ describes the relation between the operators acting on $M$ and their model operators on the osculating groups. It is equipped with a "zoom" action of $\mathbb{R}_{>0}$ that extends the dilations. In this thesis, we build the generalized fixed point algebra for the zoom action on a certain ideal $J$ in the groupoid $C^{*}$-algebra of the tangent groupoid. This generalized fixed point algebra $\operatorname{Fix}^{\mathbb{R}_{>0}}(J)$ is a $C^{*}$-subalgebra of the bounded operators on $L^{2}(M)$. Moreover, there is a "principal symbol map" which takes values in another generalized fixed point algebra $\operatorname{Fix}^{\mathbb{R}>0}\left(J_{0}\right)$, where $J_{0}$ is an ideal in the $C^{*}$-algebra of the bundle of 
osculating groups. The kernel of the symbol map consists of the compact operators. The symbol algebra is, in general, noncommutative. It is unital if $M$ is compact. In this case, call $P \in \operatorname{Fix}^{\mathbb{R}_{>0}}(J)$ elliptic if its principal symbol is invertible. We show that $P$ is elliptic if and only if all model operators satisfy the Rockland condition.

Furthermore, it is shown that the sequence above coincides with the $C^{*}$-completion of the order zero pseudodifferential extension by van Erp and Yuncken. When viewing a graded Lie group as a filtered manifold, we show that the same holds for the calculus by Fischer, Ruzhansky and Fermanian-Kammerer.

We prove that $\operatorname{Fix}^{\mathbb{R}_{>0}}\left(J_{0}\right)$ is $K K$-equivalent to the usual principal symbol algebra of functions on the cosphere bundle of $M$. Lastly, we present an index theorem, up to inverting the Connes-Thom isomorphism, for order zero pseudodifferential operators on a compact filtered manifold that are elliptic in this calculus. 


\section{Zusammenfassung}

In dieser Dissertation schlagen wir verallgemeinerte Fixpunktalgebren als Zugang zu Pseudodifferentialkalkülen auf filtrierten Mannigfaltigkeiten vor.

Eine filtrierte Mannigfaltigkeit ist eine Mannigfaltigkeit mit einer Filtrierung des Tangentialbündels, die kompatibel mit der Lieklammer ist. Diese Filtrierung ermöglicht es, einen neuen Begriff von Ordnung für die Differentialoperatoren auf $M$ zu definieren. Daraus resultiert, dass der Teil mit der höchsten Ordnung als eine Familie von rechts-invarianten Modelloperatoren auf gewissen nilpotenten Liegruppen aufgefasst werden kann. Diese Gruppen bilden das Bündel der oskulierenden Gruppen der filtrierten Mannigfaltigkeit. Die neue Ordnung kann durch eine Streckungswirkung von $\mathbb{R}_{>0}$ auf diesem Bündel beschrieben werden.

Der Tangentialgruppoid $\mathbb{T}_{H} M$ der filtrierten Mannigfaltigkeit $M$ beschreibt die Beziehung zwischen den Operatoren, die auf $M$ wirken, und ihren Modelloperatoren auf den oskulierenden Gruppen. Die Streckungen können zu einer Zoomwirkung auf $\mathbb{T}_{H} M$ erweitert werden. In dieser Arbeit konstruieren wir die verallgemeinerte Fixpunktalgebra für die Zoomwirkung auf einem Ideal $\mathbb{I}$ in der Gruppoid$\mathrm{C}^{*}$-Algebra von $\mathbb{T}_{H} M$. Diese verallgemeinerte Fixpunktalgebra $\operatorname{Fix}^{\mathbb{R}>0}(\mathbb{J})$ ist eine C*-Unteralgebra der beschränkten Operatoren auf $L^{2}(M)$. Außerdem gibt es eine Hauptsymbolabbildung $S_{H}$, die eine kurze exakte Folge induziert

$$
\mathbb{K}\left(L^{2} M\right) \longleftrightarrow \operatorname{Fix}^{\mathbb{R}>0}(\mathbb{J}) \stackrel{S_{H}}{\longrightarrow} \operatorname{Fix}^{\mathbb{R}>0}\left(\mathbb{J}_{0}\right) .
$$

Hierbei nimmt die Hauptsymbolabbildung Werte in einer weiteren verallgemeinerten Fixpunktalgebra $\operatorname{Fix}^{\mathbb{R}>0}\left(\mathbb{J}_{0}\right)$ an, wobei $\mathbb{J}_{0}$ ein Ideal in der $\mathrm{C}^{*}$-Algebra des Gruppenbündels ist. Diese Symbolalgebra ist im Allgemeinen nicht kommutativ. Sie ist unital, wenn $M$ kompakt ist. In diesem Fall nennen wir $P \in \mathrm{Fix}^{\mathbb{R}_{>0}}$ (』) elliptisch, wenn das Hauptsymbol invertierbar ist. Wir zeigen, dass $P$ genau dann elliptisch ist, wenn alle Modelloperatoren die Rocklandbedingung erfüllen.

Zudem zeigen wir, dass die obige Folge die $\mathrm{C}^{*}$-Vervollständigung der Pseudodifferentialerweiterung nullter Ordnung von van Erp und Yuncken vEY19 ist. Wenn eine graduiert nilpotente Liegruppe als filtrierte Mannigfaltigkeit aufgefasst wird, erhalten wir das gleiche Ergebnis für den Kalkül von Fischer, Ruzhansky und Fermanian-Kammerer, der in [FR16 FFK17] entwickelt wurde.

Es wird gezeigt, dass $\operatorname{Fix}^{\mathbb{R}>0}\left(\mathbb{J}_{0}\right)$ KK-äquivalent zu der gewöhnlichen Hauptsymbolalgebra von Funktionen auf dem Cosphärenbündel ist. Zuletzt beweisen wir, bis auf Invertieren des Connes-Thom-Isomorphismus, einen Indexsatz für Pseudodifferentialoperatoren nullter Ordnung auf kompakten filtrierten Mannigfaltigkeit, die elliptisch in diesem Kalkül sind. 


\section{Resumé}

I denne afhandling foreslår vi at bruge generaliserede fikspunkt-algebraer som en tilgang til pseudodifferentielle kalkyler på filtrerede mangfoldigheder.

En filtreret mangfoldighed er en mangfoldighed $M$ med en filtrering på dens tangentbundt, der er kompatibel med dens Lie-parentes. En sådan filtrering tillader os at definere et nyt ordensbegreb for differentialoperatorer på $M$, hvor højesteordensdelen er en familie af højre-invariante modeloperatorer. Disse virker på særlige nilpotente Liegrupper, som udgør bundtet af oskulerende grupper på den filtrerede mangfoldighed. Denne nye orden kan udstyres med en $\mathbb{R}_{>0}$-virkning på bundtet.

Tangentgruppoiden $\mathbb{T}_{H} M$ af en filtreret mangfoldighed $M$ beskriver relationen mellem operatorer virkende på $M$ og deres modeloperatorer på de oskulerende grupper. Denne er udstyret med en "zoom"-virkning fra $\mathbb{R}_{>0}$, som udvider dilatationerne. I denne afhandling, konstruerer vi den generaliserede fikspunkt-algebra til zoom-virkningen ud fra et bestemt ideal $\sqrt{ } \mathrm{i} \mathrm{C}^{*}$-algebra gruppoiden af $\mathbb{T}_{H} M$. Denne generaliserede fikspunkt-algebra $\operatorname{Fix}^{\mathbb{R}>0}(\mathbb{J})$ er en $\mathrm{C}^{*}$-subalgebra af de begrænsede operatorer på $L^{2}(M)$. Ydermere er der en "ledende symbol afbildning" $S_{H}$, som inducerer en kort eksakt følge

$$
\mathbb{K}\left(L^{2} M\right) \longleftrightarrow \operatorname{Fix}^{\mathbb{R}>0}(\mathbb{J}) \stackrel{S_{H}}{\longrightarrow} \operatorname{Fix}^{\mathbb{R}>0}\left(\mathbb{J}_{0}\right) .
$$

Den ledende symbol afbildning tager værdier $\mathrm{i}$ en anden generaliseret fikspunktalgebra $\operatorname{Fix}^{\mathbb{R}>0}\left(\rrbracket_{0}\right)$, hvor $\rrbracket_{0}$ er et ideal i $\mathrm{C}^{*}$-algebraen af bundtet af oskulerende grupper. Generelt vil denne symbolalgebra være ikke-kommutativ. Den har enhed såfremt $M$ er kompakt. I dette tilfælde, siger vi at $P \in \mathrm{Fix}^{\mathbb{R}>0}$ (J) er elliptisk hvis dens ledende symbol er invertibelt. Vi viser at $P$ er elliptisk hvis og kun hvis alle modeloperatorerne opfylder Rockland-betingelserne.

Det vises derudover at ovenstående følge er sammenfaldende med $\mathrm{C}^{*}$-fuldstændiggørelsen af nulteordens pseudodifferential-udvidelsen fra van Erp og Yuncken vEY19. Såfremt vi anskuer en gradueret nilpotent Liegruppe som en filtreret mangfoldighed, viser vi at det samme gør sig gældende for den kalkyle der er udviklet af Fischer, Ruzhansky og Fermanian-Kammerer [FR16 FFK17.

Vi beviser desuden at $\operatorname{Fix}^{\mathbb{R}>0}\left(\mathbb{J}_{0}\right)$ er KK-ækvivalent med den almindelige ledende symbolalgebra af funktioner på kosfærebundtet af $M$. Slutteligt præsenterer vi en indekssætning, op til inversion af Connes-Thom isomorfien, for nulteordens pseudodifferentialoperatorer på en kompakt filtreret mangfoldighed der er elliptiske i denne kalkyle. 




\section{Contents}

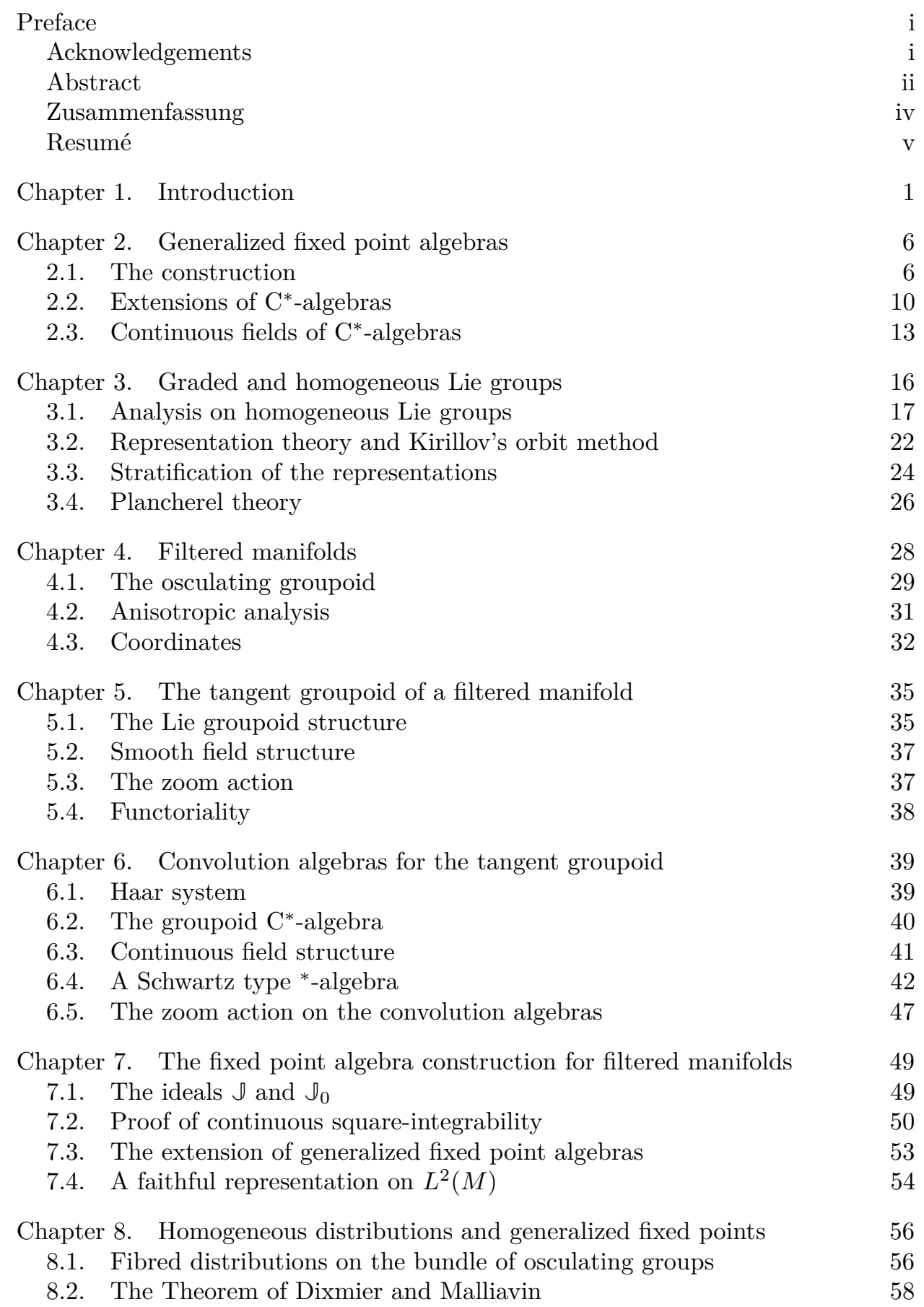


8.3. $\quad$ Operators of type 0 as generalized fixed points 60

$\begin{array}{lll}\text { Chapter 9. Comparison to calculi on filtered manifold } & 64\end{array}$

9.1. Van Erp and Yuncken's calculus 64

9.2. The calculus of Fischer, Ruzhansky and Fermanian-Kammerer $\quad 72$

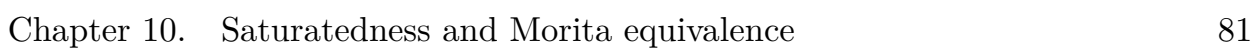

$\begin{array}{lll}\text { 10.1. Graded Lie groups } & 81\end{array}$

10.2. Filtered manifolds 83

Chapter 11. K-Theory and index theory 85

11.1. H-Ellipticity and the Rockland condition 86

11.2. Deformation to the step 1 case 88

$\begin{array}{ll}\text { 11.3. Connections to index theory } & 90\end{array}$

Chapter 12. Conclusion and outlook 93

\begin{tabular}{ll}
\hline Bibliography & 95
\end{tabular} 



\section{CHAPTER 1}

\section{Introduction}

If the kernel and cokernel of a bounded operator $P: \mathcal{H}_{1} \rightarrow \mathcal{H}_{2}$ between two Hilbert spaces are finite-dimensional, it is called a Fredholm operator. Its Fredholm index is

$$
\text { ind } P=\operatorname{dim}(\operatorname{ker} P)-\operatorname{dim}(\operatorname{coker} P) \in \mathbb{Z} \text {. }
$$

The dimension of the cokernel describes how many constraints $f \in \mathcal{H}_{2}$ has to fulfil to have a solution $x \in \mathcal{H}_{1}$ of the equation $P x=f$. The uniqueness of this solution is described by the dimension of the kernel. The difference of these integers turns out to be a useful invariant as it is, for example, stable under compact perturbations of the operator.

An elliptic differential operator on a closed manifold is Fredholm. This follows from the properties of the classical pseudodifferential calculus. The famous AtiyahSinger Index Theorem [AS68 states that its Fredholm index, which is also called the analytical index in this context, equals the more easily computed topological index.

However, there are differential operators that are Fredholm but not elliptic (see BvE14 2.3]). Consider the 3 -dimensional Heisenberg group $\mathbb{H}$ whose Lie algebra $\mathfrak{h}$ is generated by $X, Y, Z$ with the relations $[X, Y]=Z$ and $[X, Z]=[Y, Z]=0$. Let $M$ be the quotient of the Heisenberg group by the integer lattice $\Gamma$ in $\mathbb{H}$. Then the right-invariant differential operator

$$
P=-X^{2}-Y^{2}+i \mu Z
$$

is Fredholm on $M$ if and only if $\mu \in \mathbb{C} \backslash 2 \mathbb{Z}+1$. It is not elliptic, because the $Z$-part is not considered in the principal symbol as it does not belong to the highest order part. This changes if we attach different orders to the differential operators that generate $\mathfrak{h}$. If $Z$ has order 2 and $X, Y$ have order 1, all parts of $P$ contribute to the highest order part. This highest order part can be understood as a right-invariant differential operator on $\mathbb{H}$ and satisfies the Rockland condition. To formulate it, recall that each unitary, irreducible representation $\pi: \mathbb{H} \rightarrow \mathcal{U}\left(\mathcal{H}_{\pi}\right)$ of the Heisenberg group induces an infinitesimal representation $\mathrm{d} \pi$ of $\mathfrak{h}$ on the smooth vectors $\mathcal{H}_{\pi}^{\infty} \subset$ $\mathcal{H}_{\pi}$. It extends to a representation $\mathrm{d} \pi$ of the universal enveloping algebra of $\mathfrak{h}$, which can be identified with right-invariant differential operators on $\mathbb{H}$.

Definition 1.1. A right-invariant differential operator $P$ on the Heisenberg group $\mathbb{H}$ satisfies the Rockland condition if $\mathrm{d} \pi(P)$ is injective on $\mathcal{H}_{\pi}^{\infty}$ for all unitary, irreducible representations $\pi \neq \pi_{\text {triv }}$ of $\mathbb{H}$. Here, $\pi_{\text {triv }}$ denotes the trivial representation $\pi_{\text {triv }}(g)=1$ for all $g \in \mathbb{H}$.

The representation theory of the Heisenberg group is well-known. One can show that the operator above satisfies the Rockland condition, see Eps99. From this one can deduce that $P$ is Fredholm.

The idea that certain operators can be better understood when using a highest order part that acts on a nilpotent Lie group, like the Heisenberg group, goes back to Folland, Rothschild and Stein $\left[\begin{array}{l|l|l|l|}\hline \text { FS74 } & \text { RS76 } & \text { Fol77] } & \text {. It is the underlying concept of }\end{array}\right.$ 
several calculi like the ones developed in Mel82 Tay84 BG88 CGGP92 FR16 vEY19.

We may encode the different orders of the generators of $\mathfrak{h}$ by an $\mathbb{R}_{>0}$-action on $\mathfrak{h}$ that scales the generators by different powers of $r$, say $r \cdot X=r X, r \cdot Y=r Y$ and $r \cdot Z=r^{2} Z$. Then the operator above is homogeneous of degree 2. This thesis proposes to use generalized fixed point algebras of such $\mathbb{R}_{>0}$-actions to build pseudodifferential calculi.

Generalized fixed point algebras were defined by Rieffel as a noncommutative analogue of the orbit space for a proper group action $H \curvearrowright X$ on a locally compact Hausdorff space $X$. In this case, the orbit space is again locally compact and Hausdorff. Following a well-known paradigm in noncommutative geometry, one attaches to a space its $\mathrm{C}^{*}$-algebra of functions. In this case, consider $\mathrm{C}_{0}(H \backslash X)$, the continuous $\mathbb{C}$-valued functions on the orbit space $H \backslash X$ which vanish at infinity.

For example, if $H=\mathbb{R}_{>0}$ acts on $\mathbb{R}^{n} \backslash\{0\}$ by scaling, the $\mathrm{C}^{*}$-algebra of functions on the orbit space is $\mathrm{C}\left(S^{n-1}\right)$ where $S^{n-1}$ denotes the unit sphere. Equivalently, one could understand these as continuous functions that are constant along rays. Except for the zero function, they do not vanish at infinity. So they are not fixed points of the action on $\mathrm{C}_{0}\left(\mathbb{R}^{n} \backslash\{0\}\right)$. However, one could understand them as generalized fixed points as they are invariant under $\mathbb{R}_{>0}$ and still act on $\mathrm{C}_{0}\left(\mathbb{R}^{n} \backslash\{0\}\right)$ by multiplication.

To generalize this to an $H$-action on a noncommutative $\mathrm{C}^{*}$-algebra $A$, note that elements of $F \in \mathrm{C}_{0}(H \backslash X)$ can be obtained by averaging functions $f \in \mathrm{C}_{c}(X)$ over the group action by setting

$$
F(H x)=\int_{H} f\left(h^{-1} \cdot x\right) \mathrm{d} h \quad \text { for } x \in X .
$$

Therefore, one seeks a subset $\mathcal{R} \subset A$ such that averaging elements $a \in \mathcal{R}$ as above yields well-defined multipliers of $A$. These generate the generalized fixed point algebra $\operatorname{Fix}^{H}(A)$ inside the multiplier algebra of $A$. The precise construction will be recalled in this thesis. At this point, we remark that $\mathcal{R}$ can fail to exist or to be unique. Furthermore, there is a built-in Morita equivalence between $\operatorname{Fix}^{H}(A)$ and an ideal in the reduced crossed product $\mathrm{C}^{*}$-algebra $\mathrm{C}_{\mathrm{r}}^{*}(H, A)$.

For classical pseudodifferential operators on a manifold $M$, the $\mathrm{C}^{*}$-algebra of principal symbols is $\mathrm{C}_{0}\left(S^{*} M\right)$. Here, $S^{*} M$ denotes the cosphere bundle. Extending the example above, this is the $\mathrm{C}^{*}$-algebra corresponding to the orbit space of the $\mathbb{R}_{>0}$-action $\lambda \cdot(x, \xi)=(x, \lambda \xi)$ on $T^{*} M \backslash(M \times\{0\})$. Thus, it is a generalized fixed point algebra. Moreover, Debord and Skandalis observed in DS14 that the pseudodifferential operators themselves can be obtained as averages of certain elements of the $\mathrm{C}^{*}$-algebra of the tangent groupoid of $M$.

Connes' tangent groupoid 『M of a manifold $M$ (see Con94) is a continuous field of groupoids over $[0, \infty)$ given by

$$
\mathbb{} \mathbb{} \mathbb{}=(T M \times\{0\}) \cup(M \times M \times(0, \infty)) .
$$

Its groupoid structure is given by addition of tangent vectors in the fibres of $T M$, whereas the pair groupoid structure is used for $t>0$. These two components are glued together in a continuous, even smooth, way. For $M=\mathbb{R}^{n}$ the topology is such that $\left(x_{n}, y_{n}, t_{n}\right)$ converges to $(x, X, 0)$ if and only if $x_{n}, y_{n} \rightarrow x, t_{n} \rightarrow 0$ and the "difference quotient" satisfies $\left(x_{n}-y_{n}\right) / t_{n} \rightarrow X$.

As for groups, one can attach to a groupoid $\mathcal{G}$ a $\mathrm{C}^{*}$-algebra $\mathrm{C}^{*}(\mathcal{G})$ if $\mathcal{G}$ admits a Haar system. The starting point is a convolution algebra structure on $\mathrm{C}_{c}(\mathcal{G})$. For the tangent groupoid, the $\mathrm{C}^{*}$-algebra of $\mathbb{\mathbb { T }} M$ is a continuous field of $\mathrm{C}^{*}$-algebras that deforms the commutative $\mathrm{C}^{*}$-algebra $\mathrm{C}^{*}(T M)$ at $t=0$ to $\mathrm{C}^{*}(M \times M)=\mathbb{K}\left(L^{2} M\right)$ at $t=1$. 
Note that $\mathrm{C}^{*}(T M)$ is isomorphic to $\mathrm{C}_{0}\left(T^{*} M\right)$ via fibrewise Fourier transform. This allows to extend $\mathrm{C}_{0}\left(T^{*} M \backslash(M \times 0)\right)$ to an ideal $\mathbb{J}$ in $\mathrm{C}^{*}(\mathbb{T} M)$, namely the one generated by $f \in \mathrm{C}_{c}(\mathbb{T} M)$ such that $\widehat{f}_{0}(x, 0)=0$ for all $x \in M$. Here $\widehat{f}_{0}$ denotes the Fourier transform of $f_{0}$. One can extend the scaling action on $T^{*} M$ to the "zoom" action on $\mathbb{\square} M$ by setting for $\lambda>0$

$$
\begin{aligned}
\lambda \cdot(x, X, 0) & =(x, \lambda X, 0) & & \text { for } x \in M, X \in T_{x} M, \\
\lambda \cdot(x, y, t) & =\left(x, y, \lambda^{-1} t\right) & & \text { for } x, y \in M, t>0 .
\end{aligned}
$$

The $\mathrm{C}^{*}$-algebra generated by the pseudodifferential operators of order zero is isomorphic to $\mathrm{Fix}^{\mathbb{R}_{>0}}(\mathbb{J})$ for the zoom action of $\mathbb{R}_{>0}$.

In this thesis, we extend this generalized fixed point algebra construction to the situation when different orders are attached to vector fields. Filtered manifolds constitute a general framework where this is possible.

Definition 1.2. Let $M$ be a smooth manifold with a filtration of its tangent bundle $0=H^{0} \subseteq H^{1} \subseteq H^{2} \subseteq \ldots \subseteq H^{r}=T M$ by smooth subbundles $H^{i}$. The manifold is called a filtered manifold if the Lie bracket of vector fields satisfies

$$
\left[\Gamma^{\infty}\left(H^{i}\right), \Gamma^{\infty}\left(H^{j}\right)\right] \subseteq \Gamma^{\infty}\left(H^{i+j}\right) \text { for all } i, j .
$$

Here, we set $H^{i}=T M$ for all $i \geq r$.

Examples of filtered manifolds are graded Lie groups, foliations or Heisenberg manifolds, in particular, also contact manifolds. There is an associated graded vector bundle

$$
\mathfrak{t}_{H} M=\bigoplus_{i=1}^{\infty} H^{i} / H^{i-1}
$$

The condition on the Lie bracket above allows to equip each fibre with the structure of a nilpotent Lie algebra. Using the Baker-Campbell-Hausdorff formula, they integrate to nilpotent Lie groups, called the osculating groups. The graded vector bundle with this group structure in the fibres is denoted by $T_{H} M$. Following the conventions in the literature, we call it the bundle of osculating groups, even though it cannot be understood as a fibre bundle of groups in the usual sense. The reason is that the isomorphism type of the osculating groups may vary from point to point.

In the example $M=\mathbb{H} \Gamma$ from the beginning, one obtains a filtration where $H^{1}$ is generated by $X, Y$ and $H^{2}=T M$. In this case, all osculating groups are isomorphic to the Heisenberg group $\mathbb{H}$.

Extending the given example, there is a well-defined dilation action of $\mathbb{R}_{>0}$ on $\mathfrak{t}_{H} M$ given by $\lambda \cdot X=\lambda^{i} X$ for $X \in\left(H^{i} / H^{i-1}\right)_{x}$ and $x \in M$. The part of a differential operator which is homogeneous of the highest degree with respect to these dilations is called the highest order part. It can be understood as a family of right-invariant operators on the osculating groups. In the classical pseudodifferential calculus, these correspond to the model operators obtained by "freezing coefficients" at $x \in M$. These can be understood as right-invariant operators on $T_{x} M \cong \mathbb{R}^{n}$. Therefore, the bundle of osculating groups $T_{H} M$ is the right replacement for $T M$.

As in the case without a filtration, $T_{H} M$ fits into a tangent groupoid, which is adapted to the filtered structure. This tangent groupoid was constructed using different approaches in $\mathbf{v E Y 1 9}, \mathbf{C P 1 9 b}, \mathbf{S H 1 8}, \mathbf{M o h 1 8}$. It is a continuous field of groupoids over $[0, \infty)$ given by

$$
\mathbb{T}_{H} M=\left(T_{H} M \times\{0\}\right) \cup(M \times M \times(0, \infty)) .
$$

Here, the groupoid structure is such that group multiplication is used in the fibres of $T_{H} M$. Again, there is a zoom action of $\mathbb{R}_{>0}$ where we use the dilations at $t=0$. 
To define the generalized fixed point algebra one needs an appropriate ideal $\mathbb{J}$ in $\mathrm{C}^{*}\left(\mathbb{T}_{H} M\right)$. Observe that in the case without a filtration,

$$
\widehat{f}(0)=0 \Longleftrightarrow \int f(x) \mathrm{d} x=0 \Longleftrightarrow f \in \operatorname{ker}\left(\pi_{\text {triv }}: \mathrm{C}^{*}\left(\mathbb{R}^{n}\right) \rightarrow \mathbb{C}\right)
$$

for $f \in \mathcal{S}\left(\mathbb{R}^{n}\right)$. Here, $\pi_{\text {triv }}: \mathbb{R}^{n} \rightarrow \mathbb{C}$ is the trivial representation $\pi_{\text {triv }}(x)=1$ for all $x \in \mathbb{R}^{n}$. It induces a representation of $\mathrm{C}^{*}\left(\mathbb{R}^{n}\right)$ given by $f \mapsto \int_{\mathbb{R}^{n}} f(x) \mathrm{d} x$ for $f \in \mathcal{S}\left(\mathbb{R}^{n}\right)$.

Therefore, we define $\rrbracket_{0}$ to be the ideal in $\mathrm{C}^{*}\left(T_{H} M\right)$ consisting of elements that restrict at all $x \in M$ to elements that lie in the kernel of the trivial representation of the osculating group at $x$. This will replace $\mathrm{C}_{0}\left(T^{*} M \backslash(M \times 0)\right)$. The ideal $\mathbb{I}$ in $\mathrm{C}^{*}\left(\mathbb{T}_{H} M\right)$ consists of all elements whose restriction to $t=0$ lies in $\mathbb{J}_{0}$.

In this thesis, it is shown that the generalized fixed point algebra construction can be applied to the zoom action on $\mathbb{l}$. That is, we prove that a subset $\mathcal{R} \subset \mathbb{}$ with the necessary properties exists. In order to do so, we define a Schwartz type algebra $\mathcal{A}\left(\mathbb{T}_{H} M\right)$, similar to the one in $\mathbf{C R 0 8}$, by adapting the construction to filtered manifolds. It is a subalgebra of $\mathrm{C}^{*}\left(\mathbb{T}_{H} M\right)$ which consists of $f=\left(f_{t}\right) \in \mathrm{C}^{\infty}\left(\mathbb{T}_{H} M\right)$ such that $f_{t}$ are compactly supported in $M \times M$ for $t>0$ and $f_{0}$ has rapid decay in the fibres of $T_{H} M$. For the generalized fixed point algebra construction, we could also work with $\mathcal{R}=\mathrm{C}_{c}^{\infty}\left(\mathbb{T}_{H} M\right) \cap \mathbb{J}$. However, it will become apparent in later proofs that it is better to use the larger subset $\mathcal{R}=\mathcal{A}\left(\mathbb{T}_{H} M\right) \cap \mathbb{J}$.

The generalized fixed point algebra approach yields a short exact sequence

$$
\mathbb{K}\left(L^{2} M\right) \longleftrightarrow \operatorname{Fix}^{\mathbb{R}>0}(\mathbb{J}) \stackrel{S_{H}}{\longrightarrow} \operatorname{Fix}^{\mathbb{R}>0}\left(\mathbb{J}_{0}\right) .
$$

Moreover, $\operatorname{Fix}^{\mathbb{R}>0}(\mathbb{d})$ can be faithfully represented as bounded operators on $L^{2}(M)$. The sequence above can be understood as an abstract order zero pseudodifferential extension. The compact operators are the $\mathrm{C}^{*}$-completion of the operators of negative order. The algebra $\mathrm{Fix}^{\mathbb{R}>0}(\mathbb{J})$ is the completion of the order zero operators. There is a principal symbol map $S_{H}$ taking values in the $\mathrm{C}^{*}$-algebra $\operatorname{Fix}^{\mathbb{R}>0}\left(\mathbb{J}_{0}\right)$. In general, this algebra is noncommutative. It is unital if $M$ is compact. An operator $P \in \operatorname{Fix}^{\mathbb{R}_{>0}}(\mathbb{J})$ is called elliptic, if its principal symbol is invertible. In this case, $P$ is Fredholm.

We show that $\operatorname{Fix}^{\mathbb{R}>0}\left(\mathbb{J}_{0}\right)$ is a continuous field of $\mathrm{C}^{*}$-algebras over $M$. Its fibres are the generalized fixed point algebras $\operatorname{Fix}^{\mathbb{R}>0}\left(J_{x}\right)$. Here, $J_{x}$ is the kernel of the trivial representation of the osculating group $G(x)$ at $x \in M$. The elements of $\operatorname{Fix}^{\mathbb{R}_{>0}}\left(J_{x}\right)$ act as right-invariant operators on $L^{2}(G(x))$. We prove that $\operatorname{Fix}^{\mathbb{R}_{>0}}\left(J_{x}\right)$ is the $\mathrm{C}^{*}$-algebra generated by the convolution operators with kernels of type 0 that were studied in the context of homogeneous groups. For this proof, it is essential that $\mathcal{R}$ consists of Schwartz functions at $t=0$. It will be used that rapid decay is preserved by the Euclidean Fourier transform and its inverse. The generalized fixed point algebra approach yields a new proof of the result by Knapp and Stein KS71 that these operators extend to bounded operators on $L^{2}(G(x))$.

It is shown in this thesis that the sequence in (2) is the $\mathrm{C}^{*}$-closure of the order zero extension of van Erp and Yuncken $\mathbf{v E Y 1 9}$, when restricting to compactly supported kernels. Their calculus is already very close in spirit as it is also built on the tangent groupoid and the zoom action. They define a notion of $H$-ellipticity for their calculus. For a compact manifold, we show that an order zero operator $P$ in their calculus is $H$-elliptic if and only if $S_{H}(P)$ is invertible in $\operatorname{Fix}^{\mathbb{R}>0}\left(\mathbb{J}_{0}\right)$. Moreover, this is equivalent to $S_{H}(P)(x)$ and $S_{H}\left(P^{*}\right)(x)$ satisfying the Rockland condition at each $x \in M$. The generalized fixed point algebra construction allows to understand the Rockland condition in a natural way by describing the spectrum of $\operatorname{Fix}^{\mathbb{R}>0}\left(J_{x}\right)$. 
Fischer and Ruzhansky developed in FR16 a pseudodifferential calculus for graded Lie groups $G$. It is a symbolic calculus, which uses the operator-valued Fourier transform defined in terms of the representations of $G$. In FFK17 homogeneous expansions for their symbols were defined. We show that the $\mathrm{C}^{*}$-completion of their order zero pseudodifferential extension is the sequence $(2)$ for $M=G$.

By the generalized fixed point algebra construction, the algebras of pseudodifferential operators and principal symbols, $\operatorname{Fix}^{\mathbb{R}>0}(\mathbb{J})$ and $\operatorname{Fix}^{\mathbb{R}>0}\left(\mathbb{J}_{0}\right)$, are Morita equivalent to ideals in $\mathrm{C}_{\mathrm{r}}^{*}\left(\mathbb{R}_{>0}, \mathbb{J}\right)$ and $\mathrm{C}_{\mathrm{r}}^{*}\left(\mathbb{R}_{>0}, \mathbb{J}_{0}\right)$, respectively. Using the representation theory of nilpotent Lie groups, in particular, Kirillov theory Kir62 and Pukánszky's stratification Puk67, we show that they are, in fact, Morita equivalent to the whole reduced crossed products. This allows us to prove, using the Connes-Thom isomorphism, that $\operatorname{Fix}^{\mathbb{R}>0}\left(\mathbb{J}_{0}\right)$ has the same K-theory as the usual $\mathrm{C}^{*}$-algebra of symbols $\mathrm{C}_{0}\left(S^{*} M\right)$.

An index theorem for contact manifolds was accomplished by van Erp and Baum in BvE14, extending work in vE10a vE10b. Recently, Mohsen proved an index theorem for filtered manifolds in $|\mathbf{M o h 2 0}|$. In this thesis, we prove a theorem for $H$-elliptic pseudodifferential operators of order 0 on a compact filtered manifold, which reduces the index problem to inverting the Connes-Thom isomorphism.

Note that parts of this thesis, in particular the results for graded Lie groups, are contained in Ewe20.

This thesis is organized as follows. Chapter 2 gives an overview on generalized fixed point algebras. Some new results regarding their behaviour under $\mathrm{C}^{*}$-algebra extensions are proved. In Chapter 3 graded Lie groups are defined. Moreover, analysis on these groups and their representation theory is discussed. Chapter 4 is concerned with filtered manifolds. In particular, the bundle of osculating groups is defined. The construction of the tangent groupoid of a filtered manifold is recalled in Chapter 5 In Chapter 6, the $\mathrm{C}^{*}$-algebra of the tangent groupoid and the Schwartz type algebra are defined. We prove in Chapter 7 that the generalized fixed point algebra construction applies to the zoom action of $\mathbb{R}_{>0}$ on the ideal $\mathbb{I}$ in $\mathrm{C}^{*}\left(\mathbb{T}_{H} M\right)$. We obtain the sequence in (2). The relation between generalized fixed point algebras and kernels of type 0 is examined in Chapter 8 . In Chapter 9, we compare the generalized fixed point algebra construction to the calculus of van Erp and Yuncken and the calculus of Fischer, Ruzhansky and Fermanian-Kammerer. The Morita equivalence to the reduced crossed products is proved in Chapter 10 In Chapter 11. $H$-ellipticity is discussed, and we describe the results towards index theory for filtered manifolds. Chapter 12 consists of a short conclusion and an outlook. 


\section{CHAPTER 2}

\section{Generalized fixed point algebras}

Let $H$ be a locally compact group and $X$ a locally compact Hausdorff space. If $H$ acts properly on $X$, the orbit space $H \backslash X$ is again locally compact and Hausdorff. Hence, one can consider the corresponding algebra of functions $\mathrm{C}_{0}(H \backslash X)$, which consists of continuous functions $f: H \backslash X \rightarrow \mathbb{C}$ vanishing at infinity.

These are not necessarily fixed points of the induced $H$-action on $\mathrm{C}_{0}(X)$, as functions $f: X \rightarrow \mathbb{C}$ that are invariant under $H$ might not vanish at infinity. However, one can regard functions in $\mathrm{C}_{0}(H \backslash X)$ as "generalized" fixed points as they act as $H$-invariant multipliers on $\mathrm{C}_{0}(X)$. Moreover, $\mathrm{C}_{0}(H \backslash X)$ is Morita-Rieffel equivalent to an ideal in the reduced crossed product $\mathrm{C}_{\mathrm{r}}^{*}\left(H, \mathrm{C}_{0}(X)\right)$ of the corresponding $\mathrm{C}^{*}$-dynamical system. This interesting property was observed in Gre77.

To generalize this to noncommutative situations, Rieffel proposed a notion of proper group actions on $\mathrm{C}^{*}$-algebras in Rie04 Rie90. For these, it is possible to build a generalized fixed point algebra as an analogue to the functions on the orbit space. If $H$ acts properly on a $\mathrm{C}^{*}$-algebra $A$, the generalized fixed point algebra Fix $^{H}(A)$ is a subalgebra of the $H$-invariant multipliers of $A$. Moreover, there is a built-in Morita-Rieffel equivalence between $\operatorname{Fix}^{H}(A)$ and an ideal in $\mathrm{C}_{\mathrm{r}}^{*}(H, A)$.

We follow the approach to generalized fixed point algebras of [Mey01]. In the first section of this chapter, we recall the notation used there and explain the construction. The following sections are concerned with some results regarding extensions of $\mathrm{C}^{*}$-algebras and continuous fields. These will be convenient in the later chapters, where we consider actions of $H=\mathbb{R}_{>0}$ on certain $\mathrm{C}^{*}$-algebras arising from groupoids.

\subsection{The construction}

For this chapter, let $H$ be a locally compact group and $A$ a $\mathrm{C}^{*}$-algebra with a strongly continuous action $\alpha: H \rightarrow \operatorname{Aut}(A)$. The following definition and results are taken from $\mathbf{M e y 0 1}$.

Denote by $\overline{\mathrm{C}_{b}(H, A)}$ and $\mathrm{C}_{c}(H, A)$ the continuous and bounded, respectively continuous and compactly supported, $A$-valued functions on $H$. The group $H$ acts diagonally on both spaces via $(h \cdot f)(x)=\alpha_{h}\left(f\left(h^{-1} x\right)\right)$ for $h, x \in H$.

Definition 2.1. For $a \in A$ define the following bra and ket operators

$$
\begin{aligned}
\left\langle\langle a|: A \rightarrow \mathrm{C}_{b}(H, A),\right. & (\langle|a| b)(x): & =\alpha_{x}(a)^{*} b, \\
|a\rangle\rangle: \mathrm{C}_{c}(H, A) \rightarrow A, & |a\rangle\rangle f: & :=\int_{H} \alpha_{x}(a) f(x) \mathrm{d} x,
\end{aligned}
$$

where $\mathrm{d} x$ denotes a fixed Haar measure on $H$.

Both operators are $H$-equivariant and adjoint to each other with respect to the pairings $\langle a \mid b\rangle=a^{*} b$ for $a, b \in A$ and $\langle f \mid g\rangle=\int_{H} f(x)^{*} g(x) \mathrm{d} x$ for $f \in \mathrm{C}_{b}(H, A)$ and $g \in \mathrm{C}_{c}(H, A)$.

The underlying idea of the following is to restrict to a subset $\mathcal{R} \subset A$, such that for $a, b \in \mathcal{R}$ the operators $\langle\langle a|\circ| b\rangle\rangle$ and $|a\rangle\rangle \circ\langle\langle b|$ yield well-defined operators in $\mathrm{C}_{\mathrm{r}}^{*}(H, A)$ and the multiplier algebra of $A$, respectively. Then $\mathcal{R}$ is completed into 
a Morita equivalence bimodule between an ideal in $\mathrm{C}_{\mathrm{r}}^{*}(H, A)$ and the $\mathrm{C}^{*}$-algebra generated by $|a\rangle\rangle\langle\langle b|$ for $a, b \in \mathcal{R}$ inside the multiplier algebra. The latter will be defined to be the generalized fixed point algebra.

To make this precise, first recall the definition of the crossed product $\mathrm{C}_{\mathrm{r}}^{*}(H, A)$. There is a covariant representation $\left(\rho^{A}, \rho^{H}\right)$ of the $\mathrm{C}^{*}$-dynamical system $(A, H, \alpha)$ on the right Hilbert $A$-module $L^{2}(H, A)$ given by

$$
\begin{array}{ll}
\left(\rho_{a}^{A} \psi\right)(x)=\alpha_{x}(a) \psi(x) & \text { for } a \in A, x \in H, \\
\left(\rho_{y}^{H} \psi\right)(x)=\psi(x y) & \text { for } x, y \in H,
\end{array}
$$

for $\psi \in \mathrm{C}_{c}(H, A)$. Equip $\mathrm{C}_{c}(H, A)$ with the convolution and involution

$$
\begin{aligned}
(f * g)(x) & =\int_{H} f(y) \alpha_{y}\left(g\left(y^{-1} x\right)\right) \mathrm{d} y, \\
f^{*}(x) & =\alpha_{x}\left(f\left(x^{-1}\right)\right)^{*}
\end{aligned}
$$

for $x \in H$. The $I$-norm is defined by

$$
\|f\|_{I}=\max \left\{\int_{H}\|f(x)\| \mathrm{d} x, \int_{H}\left\|f^{*}(x)\right\| \mathrm{d} x\right\} .
$$

The representation $\left(\rho^{A}, \rho^{H}\right)$ integrates to a ${ }^{*}$-representation $\rho$ of $\mathrm{C}_{c}(H, A)$ with

$$
\left(\rho_{f} \psi\right)(x)=\int_{H} \alpha_{x}\left(f\left(x^{-1} y\right)\right) \psi(y) \mathrm{d} y \quad \text { for } f, \psi \in \mathrm{C}_{c}(H, A),
$$

which satisfies $\left\|\rho_{f}\right\| \leq\|f\|_{I}$ for all $f \in \mathrm{C}_{c}(H, A)$.

Definition 2.2. The reduced crossed product $\mathrm{C}_{\mathrm{r}}^{*}(H, A)$ is the norm closure of $\rho\left(\mathrm{C}_{c}(H, A)\right)$ inside $\mathbb{B}\left(L^{2}(H, A)\right)$.

Lemma 2.3. The representation $\rho^{A}$ maps to the multiplier algebra of $\mathrm{C}_{\mathrm{r}}^{*}(H, A)$. If $\left(u_{\lambda}\right)$ is an approximate identity for $A$, then $\left\|F-\rho_{u_{\lambda}}^{A} \circ F\right\| \rightarrow 0$ for each $F \in$ $\mathrm{C}_{\mathrm{r}}^{*}(H, A)$.

Proof. The first claim follows from the identity $\rho_{a}^{A} \circ \rho_{f}=\rho_{a f}$ for all $a \in A$ and $f \in \mathrm{C}_{c}(H, A)$. For the second claim note that

$$
\left\|\rho_{f}-\rho_{u_{\lambda}}^{A} \circ \rho_{f}\right\|=\left\|\rho_{f-u_{\lambda} f}\right\| \leq\left\|f-u_{\lambda} f\right\|_{I},
$$

which converges to zero for compactly supported $f$. As $\mathrm{C}_{c}(H, A)$ is dense, the same holds for arbitrary elements of $\mathrm{C}_{\mathrm{r}}^{*}(H, A)$ by continuity.

To understand $\left\langle\langle a|: A \rightarrow \mathrm{C}_{b}(H, A)\right.$ as an adjointable operator $A \rightarrow L^{2}(H, A)$ for suitable $a \in A$, we use the following definition.

Definition 2.4. Let $\left\{\chi_{i}: H \rightarrow[0,1]\right\}_{i \in I}$ be a net of continuous, compactly supported functions with $\chi_{i} \rightarrow 1$ uniformly on compact subsets. Call $f \in \mathrm{C}_{b}(H, A)$ square-integrable if and only if $\left(\chi_{i} f\right)$ converges in $L^{2}(H, A)$.

Suppose $f \in \mathrm{C}_{b}(H, A)$ is square-integrable and $\left\{\tilde{\chi}_{j}: H \rightarrow[0,1]\right\}_{j \in J}$ is another net of continuous, compactly supported functions with $\tilde{\chi}_{j} \rightarrow 1$. It is shown in Mil17, 1.13, 1.15] that $\left(\tilde{\chi}_{j} f\right)$ converges in $L^{2}(H, A)$ as well and that $\lim \left(\chi_{i} f\right)=$ $\lim \left(\tilde{\chi}_{j} f\right)$. Consequently, being square-integrable does not depend on the chosen net.

Definition 2.5. An element $a \in A$ is called square-integrable if $\left\langle\langle a| b \in \mathrm{C}_{b}(H, A)\right.$ is square-integrable for all $b \in A$. 
In this case, we understand $\left\langle\langle a|\right.$ as an operator $A \rightarrow L^{2}(H, A)$. By Mey01, $a \in A$ is square-integrable if and only if $|a\rangle\rangle$ extends to an adjointable operator $L^{2}(H, A) \rightarrow A$. We also denote it by $\left.|a\rangle\right\rangle$. Its adjoint is $\left\langle\langle a|\right.$. Let $A_{\text {si }}$ be the vector space of all square-integrable elements in $A$. It becomes a Banach space with respect to the norm

$$
\left.\|a\|_{\mathrm{si}}:=\|a\|+\|\langle\langle a|\circ| a\rangle\rangle\|^{1 / 2}=\|a\|+\||a\rangle\right\rangle \| .
$$

Definition 2.6. A subset $\mathcal{R} \subset A_{\mathrm{si}}$ is called relatively continuous if for all $a, b \in \mathcal{R}$ the operator $\langle\langle a \mid b\rangle\rangle:=\langle\langle a|\circ| b\rangle\rangle \in \mathbb{B}\left(L^{2}(H, A)\right)$ is contained in the reduced crossed product $\mathrm{C}_{\mathrm{r}}^{*}(H, A) \subset \mathbb{B}\left(L^{2}(H, A)\right)$. It is called complete if $\mathcal{R}$ is a closed linear subspace of $A_{\mathrm{si}}$ with respect to $\|\cdot\|_{\mathrm{si}}$ and satisfies $\left.|a\rangle\right\rangle\left(\mathrm{C}_{c}(H, A)\right) \subset \mathcal{R}$ for all $a \in \mathcal{R}$.

Definition 2.7. A continuously square-integrable $H-\mathrm{C}^{*}$-algebra is a $\mathrm{C}^{*}$-algebra $A$ with a strongly continuous action of a locally compact group $H$ and a dense subset $\mathcal{R} \subset A$ which is relatively continuous and complete.

Example 2.8. If $H$ acts properly on a locally compact Hausdorff space $X$, the subset $\mathrm{C}_{c}(X)$ of $\mathrm{C}_{0}(X)$ consists of square-integrable elements and is relatively continuous. Defining $\mathcal{R}$ to be the closure of $\mathrm{C}_{c}(X)$ with respect to the $\|\cdot\|_{\mathrm{si}}$-norm yields a continuously square-integrable $H$ - $\mathrm{C}^{*}$-algebra.

For an arbitrary $\mathrm{C}^{*}$-algebra $\mathrm{A}$, it can happen that there is no $\mathcal{R} \subset A$ satisfying the requirements above or that there are several ones [Mey01]. However, there is a sufficient condition that guarantees that there is a unique subset $\mathcal{R}$.

Definition 2.9. Equip the primitive ideal space of $A$ with the Jacobson topology. There is a continuous $H$-action on $\operatorname{Prim}(A)$ defined by $x \cdot P=\alpha_{x}(P)$ for $x \in H$ and $P \in \operatorname{Prim}(A)$. The $H$-C $\mathrm{C}^{*}$-algebra $A$ is called spectrally proper, if this action on the primitive ideal space is proper.

TheOrem 2.10 (Mey01, 9.4]). Let $A$ be a spectrally proper $H$-C ${ }^{*}$-algebra. Then there is a unique dense, relatively continuous and complete subset.

Definition 2.11. Let $(A, \mathcal{R})$ be a continuously square-integrable $H$-C ${ }^{*}$-algebra. Let $\mathcal{F}^{H}(A, \mathcal{R})$ be the closure of $\left.|\mathcal{R}\rangle\right\rangle \subset \mathbb{B}\left(L^{2}(H, A), A\right)$. The generalized fixed point algebra $\operatorname{Fix}^{H}(A, \mathcal{R})$ is defined as the closed linear span of $\left.|\mathcal{R}\rangle\right\rangle\langle\langle\mathcal{R}|$ in the $H$-invariant multiplier algebra $\mathcal{M}^{H}(A)$.

Since $\mathcal{R}$ is complete, there is a right $\mathrm{C}_{c}(H, A)$-module structure on $\mathcal{R}$ defined by $a * f=|a\rangle\rangle(\breve{f})$ for $a \in \mathcal{R}$ and $f \in \mathrm{C}_{c}(H, A)$, where ${ }^{\triangleleft}: \mathrm{C}_{c}(H, A) \rightarrow \mathrm{C}_{c}(H, A)$ is given by $\breve{f}(h):=\alpha_{h}\left(f\left(h^{-1}\right)\right)$ for $h \in H$. Because of the identity $\left.\left.|a\rangle\right\rangle \circ \rho_{f}=|a * f\rangle\right\rangle$ for $a \in \mathcal{R}$ and $f \in \mathrm{C}_{c}(H, A)$, this can be extended continuously to a right Hilbert $\mathrm{C}_{\mathrm{r}}^{*}(H, A)$-module structure on $\mathcal{F}^{H}(A, \mathcal{R})$.

For $a, b, c, d \in \mathcal{R}$ the operator $\langle\langle b \mid c\rangle\rangle \in \mathrm{C}_{\mathrm{r}}^{*}(H, A)$ can be approximated by a sequence $\left(\rho_{f_{n}}\right)$ with $f_{n} \in \mathrm{C}_{c}(H, A)$. Therefore, the product

$$
\left.(|a\rangle\rangle\langle\langle b|)(|c\rangle\rangle\langle\langle d|)=\lim _{n \rightarrow \infty}|a\rangle\right\rangle \circ \rho_{f_{n}} \circ\left\langle\left\langle d\left|=\lim _{n \rightarrow \infty}\right| a * f_{n}\right\rangle\right\rangle\langle\langle d|
$$

lies again in the generalized fixed point algebra. As $\left.(|a\rangle\rangle\langle\langle b|)^{*}=|b\rangle\right\rangle\langle\langle a|$, this shows that $\operatorname{Fix}^{H}(A, \mathcal{R})$ is a $\mathrm{C}^{*}$-subalgebra of $\mathcal{M}^{H}(A)$.

Now, we describe the elements of $\operatorname{Fix}^{H}(A, \mathcal{R})$ more explicitly. In the commutative case $H \curvearrowright X$, functions on the orbit space can be obtained by averaging functions in $\mathrm{C}_{c}(X)$ over the action:

Example 2.12. For a proper action $H \curvearrowright X$ and $f \in \mathrm{C}_{c}(X)$ there is a function $F \in \mathrm{C}_{0}(H \backslash X)$ defined by

$$
F(H x):=\int_{H} f\left(h^{-1} \cdot x\right) \mathrm{d} h \quad \text { for } H x \in H \backslash X .
$$


The following lemma suggests to also think of elements of $\operatorname{Fix}^{H}(A, \mathcal{R})$ for a noncommutative $A$ as averages over the group action of certain elements of $A$.

Lemma 2.13 (Mey01 (19)]). Let $\left(\chi_{i}\right)_{i \in I}$ be a net of continuous, compactly supported functions on $H$ that converges uniformly to 1 on compact subsets as above. Let $a, b \in \mathcal{R}$. The net

$$
\int_{H} \chi_{i}(x) \alpha_{x}\left(a^{*} b\right) \mathrm{d} x
$$

converges to $|a\rangle\rangle\langle\langle b|$ with respect to the strict topology as multipliers of $A$.

Returning to the construction, $\mathcal{F}^{H}(A, \mathcal{R})$ is a full left Hilbert $\operatorname{Fix}^{H}(A, \mathcal{R})$ module. Let $J^{H}(A, \mathcal{R})$ denote the closed linear span of $\langle\langle\mathcal{R} \mid \mathcal{R}\rangle\rangle \subset \mathrm{C}_{\mathrm{r}}^{*}(H, A)$, which is an ideal. Then $\mathcal{F}^{H}(A, \mathcal{R})$ is a $\operatorname{Fix}^{H}(A, \mathcal{R})-J^{H}(A, \mathcal{R})$ imprimitivity bimodule.

The ideal $J^{H}(A, \mathcal{R})$ need not be the whole reduced crossed product. The following definition is due to Rieffel [Rie90.

Definition 2.14. Let $(A, \mathcal{R})$ be a continuously square-integrable $H$-C $\mathrm{C}^{*}$-algebra. Call $(A, \mathcal{R})$ saturated if $J^{H}(A, \mathcal{R})=\mathrm{C}_{\mathrm{r}}^{*}(H, A)$.

Example 2.15. For a proper action $H \curvearrowright X$, Rieffel observed in Rie82 that $\left(\mathrm{C}_{0}(X), \overline{\mathrm{C}_{c}(X)}\right)$ is saturated if the action of $H$ on $X$ is free. We will argue in Lemma 2.23 that the converse is true as well.

The next lemma, proved already in [Mil17], gives a criterion when a set $\mathcal{R} \subset$ $A_{\text {si }}$ can be completed to a dense, relatively continuous and complete subset of $A$.

Lemma 2.16. Let $\mathcal{R} \subset A$ be a dense subalgebra. Suppose $\mathcal{R}$ consists of squareintegrable elements, is relatively continuous and $H$-invariant. Denote by $\overline{\mathcal{R}}$ the closure of $\mathcal{R} \subset A_{\mathrm{si}}$ with respect to the $\|\cdot\|_{\mathrm{si}}$-norm.

Then $(A, \overline{\mathcal{R}})$ is a continuously square-integrable $H$-C $\mathrm{C}^{*}$-algebra and $\operatorname{Fix}^{H}(A, \overline{\mathcal{R}})$ is the closed linear span of $|\mathcal{R}\rangle\rangle\langle\langle\mathcal{R}|$.

Proof. The inclusion $A_{\mathrm{si}} \hookrightarrow A$ is continuous. Since $\mathcal{R}$ is dense in $A$, also $\overline{\mathcal{R}}$ is a dense subspace of $A$. As $\|\langle\langle a|\|=\|| a\rangle\rangle\| \leq\|a\|_{\text {si }}$ for all $a \in A_{\text {si }}$, elements of $\langle\langle\overline{\mathcal{R}} \mid \overline{\mathcal{R}}\rangle\rangle$ can be approximated with respect to the operator norm on $L^{2}(H, A)$ by elements of $\langle\langle\mathcal{R} \mid \mathcal{R}\rangle\rangle$. This shows that $\overline{\mathcal{R}}$ is relatively continuous as well.

It remains to verify that $\overline{\mathcal{R}}$ is complete. First, we show that $\overline{\mathcal{R}} \cdot A \subset \overline{\mathcal{R}}$ holds. Let $r \in \overline{\mathcal{R}}$ and $a \in A$ and choose sequences $\left(r_{n}\right),\left(a_{n}\right)$ in $\mathcal{R}$ such that $\left\|r-r_{n}\right\|_{\mathrm{si}} \rightarrow 0$ and $\left\|a-a_{n}\right\| \rightarrow 0$. Then $r a \in A_{\mathrm{si}}$ because $\left.\left.|r a\rangle\right\rangle=|r\rangle\right\rangle \circ \rho_{a}^{A}$ and $r$ is square-integrable. By assumption, $r_{n} a_{n} \in \mathcal{R}$ holds for all $n \in \mathbb{N}$. We estimate using [Mey01, (17)] that

$$
\left\|r a-r_{n} a_{n}\right\|_{\mathrm{si}} \leq\|r\|_{\mathrm{si}}\left\|a_{n}-a\right\|+\left\|r-r_{n}\right\|_{\mathrm{si}}\left\|a_{n}\right\| .
$$

This converges to zero. Furthermore, $\overline{\mathcal{R}}$ is also $H$-invariant, which follows from the invariance of $\mathcal{R}$ and $\mid$ Mey01, (18)]. This implies that $|\overline{\mathcal{R}}\rangle\rangle\left(\mathrm{C}_{c}(H, A)\right) \subset \overline{\mathcal{R}}$.

Using similar arguments as for the relative continuity of $\overline{\mathcal{R}}$, one obtains that any $|a\rangle\rangle\langle\langle b|$ with $a, b \in \overline{\mathcal{R}}$ is a norm limit of elements in $\mid \mathcal{R}\rangle\rangle\langle\langle\mathcal{R}|$.

Remark 2.17. Suppose $\mathcal{R} \subset A$ is a dense, $H$-invariant ${ }^{*}$-subalgebra such that $\langle\langle a| b$ is bounded with respect to the $I$-norm for all $a, b \in \mathcal{R}$ as required in the original definition in Rie90. Then by Mey01, 6.8] $\mathcal{R}$ is relatively continuous and square-integrable. Therefore, Lemma 2.16 shows that $(A, \overline{\mathcal{R}})$ is a continuously square-integrable $H$-C ${ }^{*}$-algebra. 


\subsection{Extensions of $\mathrm{C}^{*}$-algebras}

Let $I$ be an $H$-invariant ideal in $A$ such that the sequence

$$
\mathrm{C}_{\mathrm{r}}^{*}(H, I) \longleftrightarrow \mathrm{C}_{\mathrm{r}}^{*}(H, A) \longrightarrow \mathrm{C}_{\mathrm{r}}^{*}(H, A / I)
$$

is exact. If $H$ is an exact group, this is true for all $H$-invariant ideals $I \triangleleft A$. For example, this holds in our applications in the later chapters where $H=\mathbb{R}_{>0} \cong \mathbb{R}$.

Let $\mathcal{R} \subset A$ be a subset such that $(A, \mathcal{R})$ is a continuously square-integrable $H$-C ${ }^{*}$-algebra. Consider $\mathcal{R} \cap I \subset I$ and the image of $\mathcal{R}$ under the projection $q: A \rightarrow$ $A / I$. We are going to show that the generalized fixed point algebra construction can be applied to $(I, \mathcal{R} \cap I)$ and $(A / I, \overline{q(\mathcal{R})})$, and to relate the respective generalized fixed point algebras to each other.

In particular, we are interested in what can be said about saturatedness in this case. This is inspired by the simple observation that if an $H$-space $X$ can be partitioned into two $H$-invariant subsets $X=X_{1} \sqcup X_{2}$, then the action on $X$ is free if and only if it is free on $X_{1}$ and $X_{2}$.

Lemma 2.18 ( $(\mathbf{M i l 1 7}])$. Let $\mathcal{R} \subseteq A$ be a relatively continuous, complete subspace of $A$. If $I \triangleleft A$ is an $H$-invariant ideal such that (3) is exact, then $\mathcal{R} \cap I=\mathcal{R} \cdot I$ holds.

Proof. Because $I$ is an ideal in $A$ and $\mathcal{R} \cdot A=\mathcal{R}$ by Mey01, Cor. 6.7], $\mathcal{R} \cdot I \subseteq \mathcal{R} \cap I$ follows. The other inclusion uses exactness in $(3)$. Let $r \in \mathcal{R} \cap I$. As

$$
\langle\langle r \mid r\rangle\rangle\left(L^{2}(H, A)\right) \subseteq L^{2}(H, I)
$$

and (3) is exact, we have $\langle\langle r \mid r\rangle\rangle \in \mathrm{C}_{\mathrm{r}}^{*}(H, I)$. Now, let $\left(u_{\lambda}\right)_{\lambda \in \Lambda}$ be an approximate unit for $I$, satisfying $u_{\lambda}^{*}=u_{\lambda}$ and $\left\|u_{\lambda}\right\| \leq 1$ for all $\lambda \in \Lambda$. One computes

$$
\begin{aligned}
\left.\||r\rangle\rangle-\left|r u_{\lambda}\right\rangle\right\rangle \|^{2} & =\left\|\left\langle\left\langle r-r u_{\lambda} \mid r-r u_{\lambda}\right\rangle\right\rangle\right\| \\
& \leq\left\|\langle\langle r \mid r\rangle\rangle-\rho_{u_{\lambda}^{*}}^{I} \circ\langle\langle r \mid r\rangle\rangle\right\|+\left\|\langle\langle r \mid r\rangle\rangle \circ \rho_{u_{\lambda}}^{I}-\rho_{u_{\lambda}^{*}}^{I} \circ\langle\langle r \mid r\rangle\rangle \circ \rho_{u_{\lambda}}^{I}\right\| \\
& \leq 2 \cdot\left\|\langle\langle r \mid r\rangle\rangle-\rho_{u_{\lambda}}^{I} \circ\langle\langle r \mid r\rangle\rangle\right\| .
\end{aligned}
$$

By Lemma 2.3 this converges to zero. Furthermore, $\left\|r-r u_{\lambda}\right\| \rightarrow 0$ holds. Hence, $r \in \mathcal{R} \cdot I$ follows from Cohen's Factorization Theorem applied to $\left(\mathcal{R},\|\cdot\|_{\mathrm{si}}\right)$ as a right $I$-module.

Lemma 2.19. Let $(A, \mathcal{R})$ be a continuously square-integrable $H$ - $\mathrm{C}^{*}$-algebra and let $I \triangleleft A$ be an $H$-invariant ideal such that the sequence of the reduced crossed products in (3) is exact. Let $q: A \rightarrow A / I$ be the quotient map. Then the following holds:

(i) $(I, \mathcal{R} \cap I)$ is a continuously square-integrable $H-\mathrm{C}^{*}$-algebra.

(ii) $(A / I, \overline{q(\mathcal{R})})$ is a continuously square-integrable $H-\mathrm{C}^{*}$-algebra. Here, $\overline{q(\mathcal{R})}$ denotes the closure of $q(\mathcal{R}) \subset(A / I)_{\mathrm{si}}$ in the $\|\cdot\|_{\mathrm{si}}-$ norm.

Proof. We prove(i) The linear subspace $\mathcal{R} \cap I=\mathcal{R} \cdot I$ is dense in $I$ because any element $i \in I$ can be factorized as $i=a \cdot j$ for some $a \in A$ and $j \in I$. Since $\mathcal{R}$ is dense in $A$, there is a net $\left(r_{\lambda}\right)_{\lambda \in \Lambda} \subset \mathcal{R}$ with $r_{\lambda} \rightarrow a$ and hence $i=$ $\lim _{\lambda} r_{\lambda} \cdot j$. The square-integrability of elements in $\mathcal{R} \cap I$ is inherited from $\mathcal{R}$, and $|\mathcal{R} \cap I\rangle\rangle\left(\mathrm{C}_{c}(H, I)\right) \subseteq \mathcal{R} \cap I$ holds. Then $\langle\langle\mathcal{R} \cap I \mid \mathcal{R} \cap I\rangle\rangle \subset \mathrm{C}_{\mathrm{r}}^{*}(H, I)$ follows from the same argument as in the proof of Lemma 2.18 using that (3) is exact. Note that $\|\langle\langle i \mid i\rangle\rangle\|_{\mathrm{C}_{\mathrm{r}}^{*}(H, I)}=\|\langle\langle i \mid i\rangle\rangle\|_{\mathrm{C}_{\mathrm{r}}^{*}(H, A)}$ for $i \in \mathcal{R} \cap I$. Because $I \triangleleft A$ is closed and $\mathcal{R}$ is closed with respect to $\|\cdot\|_{\mathrm{si}, A}$, this means that $\mathcal{R} \cap I$ is closed with respect to $\|\cdot\|_{\mathrm{si}, I}$. Hence, $(I, \mathcal{R} \cap I)$ is a continuously square-integrable $H$-C $\mathrm{C}^{*}$-algebra.

To prove (ii) we show that Lemma 2.16 can be applied to $q(\mathcal{R}) \subset A / I$. As $\mathcal{R} \subset A$ is a dense linear subspace, the same holds for $q(\mathcal{R}) \subset A / I$. For $a \in \mathcal{R}$ and $i \in I$ their product $a i \in \mathcal{R} \cdot I=\mathcal{R} \cap I$ lies in $\mathcal{R}$. All elements $q(a)$ for $a \in \mathcal{R}$ are 
square-integrable because the quotient map $L^{2}(H, A) \rightarrow L^{2}(H, A / I)$ is continuous. Let $Q: \mathbb{B}\left(L^{2}(H, A)\right) \rightarrow \mathbb{B}\left(L^{2}(H, A / I)\right)$ be the canonical map. We have

$$
\langle\langle q(a) \mid q(b)\rangle\rangle=Q(\langle\langle a \mid b\rangle\rangle) \quad \text { for } a, b \in \mathcal{R} .
$$

The relative continuity of $q(\mathcal{R})$ follows as $Q$ maps $\mathrm{C}_{\mathrm{r}}^{*}(H, A)$ to $\mathrm{C}_{\mathrm{r}}^{*}(H, A / I)$. By Mey01, 6.7], $\mathcal{R}$ is $H$-invariant and an essential right $A$-module, that is, $\mathcal{R} \cdot A=$ $\mathcal{R}$. This implies that $q(\mathcal{R})$ is also $H$-invariant and satisfies $q(\mathcal{R}) \cdot q(\mathcal{R}) \subset q(\mathcal{R})$. Therefore, the claim follows from Lemma 2.16

Remark 2.20. The restricted map $q: A_{\mathrm{si}} \rightarrow(A / I)_{\mathrm{si}}$ is continuous with respect to the respective $\|\cdot\|_{\mathrm{si}}$-norms as for $a \in A_{\mathrm{si}}$

$$
\|q(a)\|+\|\langle\langle q(a) \mid q(a)\rangle\rangle\|^{1 / 2}=\|q(a)\|+\|Q(\langle\langle a \mid a\rangle\rangle)\|^{1 / 2} \leq\|a\|+\|\langle\langle a \mid a\rangle\rangle\| .
$$

If $\mathcal{R} \subset A$ is the closure of some $\mathcal{R}_{0} \subset A$ with respect to the $\|\cdot\|_{\mathrm{si}}$-norm, then $\overline{q(\mathcal{R})}=\overline{q\left(\overline{\mathcal{R}_{0}}\right)}=\overline{q\left(\overline{\mathcal{R}_{0}}\right)}$ because $q$ is continuous with respect to the $\|\cdot\|_{\mathrm{si}}$-norms.

In the situation of Lemma 2.19 $\mathcal{F}^{H}(I, \mathcal{R} \cap I)$ is a closed $\operatorname{Fix}^{H}(A, \mathcal{R})-J^{H}(A, \mathcal{R})$ submodule of $\mathcal{F}^{H}(A, \mathcal{R})$. Under the Rieffel correspondence (see for example RW98, 3.22]), $\mathcal{F}^{H}(I, \mathcal{R} \cap I)$ corresponds to the ideals $\operatorname{Fix}^{H}(I, \mathcal{R} \cap I)$ in $\operatorname{Fix}^{H}(A, \mathcal{R})$ and $J^{H}(I, \mathcal{R} \cap I)$ in $J^{H}(A, \mathcal{R})$.

To study saturatedness, we relate the corresponding ideals in the reduced crossed products for $I, A$ and $A / I$.

Lemma 2.21. Let $(A, \mathcal{R})$ be a continuously square-integrable $H$-C*-algebra and $I \triangleleft A$ an $H$-invariant ideal such that $(3)$ is exact.

The restrictions of $\mathrm{C}_{\mathrm{r}}^{*}(H, I) \rightarrow \mathrm{C}_{\mathrm{r}}^{*}(H, A)$ and $Q: \mathrm{C}_{\mathrm{r}}^{*}(H, A) \rightarrow \mathrm{C}_{\mathrm{r}}^{*}(H, A / I)$ to $J^{H}(I, \mathcal{R} \cap I)$ and $J^{H}(A, \mathcal{R})$, respectively, yield a commutative diagram with exact rows

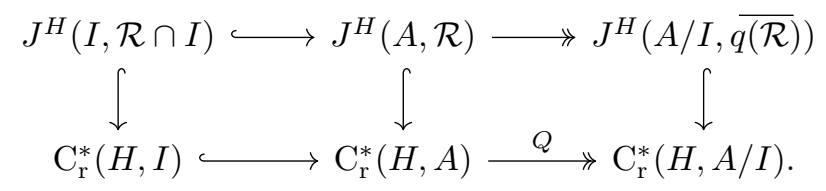

Proof. The ideal $J^{H}(I, \mathcal{R} \cap I)$ is mapped into $J^{H}(A, \mathcal{R})$ under the inclusion. As $Q(\langle\langle a \mid b\rangle\rangle)=\langle\langle q(a) \mid q(b)\rangle\rangle$ for $a, b \in \mathcal{R}$, it follows that $J^{H}(A, \mathcal{R})$ maps to $J^{H}(A / I, \overline{q(\mathcal{R})})$. Moreover, the linear span of elements of this form is dense in $J^{H}(A / I, \overline{q(\mathcal{R})})$ so that the restriction is onto. Hence, the claim follows from exactness of the bottom row in (4) once we show that $J^{H}(I, \mathcal{R} \cap I)=J^{H}(A, \mathcal{R}) \cap \mathrm{C}_{\mathrm{r}}^{*}(H, I)$.

As $J^{H}(A, \mathcal{R})$ and $\mathrm{C}_{\mathrm{r}}^{*}(H, I)$ are both closed ideals in $\mathrm{C}_{\mathrm{r}}^{*}(H, A)$,

$$
J^{H}(A, \mathcal{R}) \cap \mathrm{C}_{\mathrm{r}}^{*}(H, I)=J^{H}(A, \mathcal{R}) \cdot \mathrm{C}_{\mathrm{r}}^{*}(H, I)
$$

holds. Consequently, the linear span of $\langle\langle a \mid b\rangle\rangle \circ \rho_{f}=\langle\langle a \mid b * f\rangle\rangle$ for $a, b \in \mathcal{R}$ and $f \in \mathrm{C}_{c}(H, I)$ is dense in $J^{H}(A, \mathcal{R}) \cap \mathrm{C}_{\mathrm{r}}^{*}(H, I)$. Let $\left(u_{\lambda}\right)_{\lambda \in \Lambda}$ be a approximate unit for $I$ consisting of self-adjoint $u_{\lambda}$. Lemma 2.3 implies that $\langle\langle a \mid b * f\rangle\rangle$ is the limit of $\rho_{u_{\lambda}} \circ\langle\langle a \mid b * f\rangle\rangle=\left\langle\left\langle a u_{\lambda} \mid b * f\right\rangle\right\rangle$. This net lies in $J^{H}(I, \mathcal{R} \cap I)$ as $a u_{\lambda} \in \mathcal{R} \cdot I=\mathcal{R} \cap I$ and $b * f \in \mathcal{R} \cap I$. Thus, the inclusion $J^{H}(A, \mathcal{R}) \cap \mathrm{C}_{\mathrm{r}}^{*}(H, I) \subseteq J^{H}(I, \mathcal{R} \cap I)$ follows. The converse inclusion is clear.

Corollary 2.22. Let $(A, \mathcal{R})$ be a continuously square-integrable $H-\mathrm{C}^{*}$-algebra and $I \triangleleft A$ an $H$-invariant ideal such that (3) is exact. Then $(A, \mathcal{R})$ is saturated if and only if $(I, \mathcal{R} \cap I)$ and $(A / I, \overline{q(\mathcal{R})})$ are saturated.

Proof. Suppose first that $(A, \mathcal{R})$ is saturated. In the proof of Lemma 2.21 we showed that $J^{H}(I, \mathcal{R} \cap I)=J^{H}(A, \mathcal{R}) \cap \mathrm{C}_{\mathrm{r}}^{*}(H, I)$. Hence $(I, \mathcal{R} \cap I)$ is saturated. Because (4) has exact rows, this implies that $(A / I, \overline{q(\mathcal{R})})$ is saturated as well. If $(I, \mathcal{R} \cap I)$ and $(A / I, \overline{q(\mathcal{R})})$ are saturated, $(A, \mathcal{R})$ is saturated because $(4)$ is exact. 
As an application we show the following result for actions on spaces.

Lemma 2.23. Let $H$ act properly on a locally compact Hausdorff space $X$ and assume $\left(\mathrm{C}_{0}(X), \overline{\mathrm{C}_{c}(X)}\right)$ is saturated. Then the action $H \curvearrowright X$ is free.

Proof. Let $x \in X$ and let $H x \subseteq X$ be its orbit. Since the action is proper, $H x$ is $H$-equivariantly homeomorphic to $H / H_{x}$. Here $H_{x}$ is the stabilizer of $x$, which is a compact subgroup of $H$. Hence, $\mathrm{C}_{0}(H x)$ is a quotient of $\mathrm{C}_{0}(X)$ by an $H$-invariant ideal. Because $\mathrm{C}_{0}(H x)$ is spectrally proper, $\left.\overline{\mathrm{C}_{c}(H x}\right)$ is the unique relatively continuous, complete and dense subset by Theorem 2.10 By Corollary 2.22, $\left.\left(\mathrm{C}_{0}(H x), \overline{\mathrm{C}_{c}(H x}\right)\right)$ is saturated. Hence, Fix $^{H}\left(\mathrm{C}_{0}(H x), \overline{\mathrm{C}_{c}(H x)}\right)$ is Morita-Rieffel equivalent to $\mathrm{C}_{\mathrm{r}}^{*}\left(H, \mathrm{C}_{0}(H x)\right)$. By the Imprimitivity Theorem, $\mathrm{C}_{\mathrm{r}}^{*}\left(H, \mathrm{C}_{0}\left(H / H_{x}\right)\right)$ is Morita-Rieffel equivalent to $\mathrm{C}^{*}\left(H_{x}\right)$.

On the other hand, $\left.\operatorname{Fix}^{H}\left(\mathrm{C}_{0}(H x), \overline{\mathrm{C}_{c}(H x}\right)\right)$ is isomorphic to the functions on the orbit space. As $H x$ consists of a single $H$-orbit, this generalized fixed point algebra is isomorphic to $\mathbb{C}$. Hence, $\mathbb{C}$ and $\mathrm{C}^{*}\left(H_{x}\right)$ are Morita-Rieffel equivalent. This can only be true if $H_{x}=\{e\}$. Therefore, the $H$-action on $X$ is free.

Not only the ideals in the crossed product algebras fit into an exact sequence. The same is true for the corresponding generalized fixed point algebras. The surjective homomorphism $q: A \rightarrow A / I$ has a unique strictly continuous extension $\mathcal{M}(A) \rightarrow \mathcal{M}(A / I)$. Let $\widetilde{q}$ be its restriction to $\operatorname{Fix}^{H}(A, \mathcal{R})$.

Proposition 2.24. Let $(A, \mathcal{R})$ be a continuously square-integrable $H$-C ${ }^{*}$-algebra and $I \triangleleft A$ an $H$-invariant ideal such that (3) is exact. There is an extension of generalized fixed point algebras

$$
\operatorname{Fix}^{H}(I, \mathcal{R} \cap I) \longleftrightarrow \operatorname{Fix}^{H}(A, \mathcal{R}) \stackrel{\widetilde{q}}{\longrightarrow} \operatorname{Fix}^{H}(A / I, \overline{q(\mathcal{R})}) .
$$

Proof. For $a, b \in \mathcal{R} \cap I$, we can view $|a\rangle\rangle\langle\langle b|$ as a multiplier of $I$ or $A$. As $|a\rangle\rangle\langle\langle b|(A) \subset I$ it follows that $\| \mid a\rangle\rangle\left\langle\left\langle b\left|\left\|_{I}=\right\|\right| a\right\rangle\right\rangle\left\langle\langle b| \|_{A}\right.$. Hence, by extending continuously we obtain an injective *-homomorphism $\operatorname{Fix}^{H}(I, \mathcal{R} \cap I) \rightarrow \operatorname{Fix}^{H}(A, \mathcal{R})$. imply

Denote by $\beta$ the induced $H$-action on $A / I$. Strict continuity of $\widetilde{q}$ and Lemma 2.13

$$
\left.\widetilde{q}(|a\rangle\rangle\langle\langle b|)=\lim _{s} \int_{H} q\left(\alpha_{x}\left(a^{*} b\right)\right) \mathrm{d} x=\lim _{s} \int_{H} \beta_{x}\left(q\left(a^{*} b\right)\right) \mathrm{d} x=|q(a)\rangle\right\rangle\langle\langle q(b)|
$$

for $a, b \in \mathcal{R}$. This shows that the image of $\widetilde{q}$ is contained in $\operatorname{Fix}^{H}(A, \overline{q(\mathcal{R})})$. Moreover, the linear span of elements of this form is dense in $\operatorname{Fix}^{H}(A, \overline{q(\mathcal{R})})$. So $\widetilde{q}$ is onto.

It remains to show that the kernel of $\widetilde{q}$ is $\operatorname{Fix}^{H}(I, \mathcal{R} \cap I)$. The computation above yields $\widetilde{q}(|a\rangle\rangle\langle\langle b|)=|q(a)\rangle\rangle\left\langle\langle q(b)|=0\right.$ for $a, b \in \mathcal{R} \cap I$. Thus, $\operatorname{Fix}^{H}(I, \mathcal{R} \cap I)$ is contained in $\operatorname{ker}(\widetilde{q})$. Let $T \in \operatorname{Fix}^{H}(A, \mathcal{R})$ be such that $\widetilde{q}(T)=0$. By the $\mathrm{C}^{*}$-identity in $\operatorname{Fix}^{H}(A, \mathcal{R}) / \operatorname{Fix}^{H}(I, \mathcal{R} \cap I)$ it will suffice to show that $T^{*} T \in \operatorname{Fix}^{H}(I, \mathcal{R} \cap I)$. By Mey01, (13)], $\left.T^{*}|a\rangle\right\rangle\left\langle\left\langle b|=| T^{*} a\right\rangle\right\rangle\left\langle\langle b|\right.$ holds for $a, b \in \mathcal{R}$. As $T^{*} a$ is square-integrable and $\left.\left.\left|T^{*} a\right\rangle\right\rangle=T^{*}|a\rangle\right\rangle \in \operatorname{Fix}^{H}(A, \mathcal{R}) \cdot \mathcal{F}^{H}(A, \mathcal{R}) \subseteq \mathcal{F}^{H}(A, \mathcal{R})$, Mey01, 6.5] implies $T^{*} a \in \mathcal{R}$. Moreover, $q\left(T^{*} a\right)=\widetilde{q}\left(T^{*}\right) q(a)=0$ shows that $T^{*} a \in \mathcal{R} \cap I$. The equalities $\mathcal{R} \cap I=\mathcal{R} \cdot I$ and $I=I^{2}$ imply that there are $c \in \mathcal{R}$ and $i, j \in I$ with $T^{*} a=c i j$. The computation

$$
\left.|c i j\rangle\rangle\left\langle\left\langle b \mid=(|c i\rangle\rangle \circ \rho_{j}\right) \circ\langle\langle b|=| c i\rangle\rangle(|b\rangle\rangle \circ \rho_{j^{*}}\right)^{*}=|c i\rangle\right\rangle\left\langle\left\langle b j^{*}\right|\right.
$$

shows that $\left.T^{*}|a\rangle\right\rangle\left\langle\langle b| \in \operatorname{Fix}^{H}(I, \mathcal{R} \cap I)\right.$. By definition of the generalized fixed point algebra, $T$ is the limit of a sequence in the linear span of $|\mathcal{R}\rangle\rangle\langle\langle\mathcal{R}|$. Hence, it follows that $T^{*} T \in \operatorname{Fix}^{H}(I, \mathcal{R} \cap I)$. 


\subsection{Continuous fields of $\mathrm{C}^{*}$-algebras}

We end this chapter with some results on generalized fixed point algebras of continuous fields of $\mathrm{C}^{*}$-algebras. Recall the definition of continuous fields of $\mathrm{C}^{*}$-algebras and $\mathrm{C}_{0}(X)$-algebras.

Definition 2.25. Let $X$ be a locally compact Hausdorff space. A $\mathrm{C}_{0}(X)$-algebra is a $\mathrm{C}^{*}$-algebra $A$ with a non-degenerate injective homomorphism $\theta: \mathrm{C}_{0}(X) \rightarrow$ $Z \mathcal{M}(A)$ into the centre of the multiplier algebra of $A$.

Definition 2.26. Let $X$ be a locally compact Hausdorff space and $\left(A_{x}\right)_{x \in X}$ a family of non-zero $\mathrm{C}^{*}$-algebras. A family $a=\left(a_{x}\right)_{x \in X}$ of $a_{x} \in A_{x}$ is called a section. An upper semi-continuous of field of $\mathrm{C}^{*}$-algebras is a triple $\left(X,\left(A_{x}\right)_{x \in X}, A\right)$, where $A$ consists of sections and satisfies

(i) $A$ is a $\mathrm{C}^{*}$-algebra with respect to the pointwise operations and the norm $\|a\|=\sup _{x \in X}\left\|a_{x}\right\|$

(ii) $A_{x}=\left\{a_{x} \mid a \in A\right\}$ for all $x \in X$,

(iii) for each $a \in A$ the map $x \mapsto\left\|a_{x}\right\|$ vanishes at infinity and is upper semicontinuous,

(iv) for each $f \in \mathrm{C}_{0}(X)$, the section $f \cdot a$ defined by $(f \cdot a)_{x}=f(x) a_{x}$ is in $A$. It is called a continuous field of $\mathrm{C}^{*}$-algebras if the maps $x \mapsto\left\|a_{x}\right\|$ in (iii) belong to $\mathrm{C}_{0}(X)$ for all $a \in A$.

It is well-known that every upper semi-continuous field of $\mathrm{C}^{*}$-algebras over $X$ is a $\mathrm{C}_{0}(X)$-algebra using (iv) Conversely each $\mathrm{C}_{0}(X)$-algebra defines an upper semi-continuous field of $\mathrm{C}^{*}$-algebras with fibres $A_{x}=A / \overline{I_{x} \cdot A}$, where $I_{x}$ is the ideal of functions in $\mathrm{C}_{0}(X)$ that vanish at $x \in X$ (see $\left.\mathbf{N i l 9 6}, 2.3\right]$ ).

Definition 2.27 ( $\mathbf{K a s 8 8}]$ ). Let $H$ act on a locally compact Hausdorff space $X$. Denote by $\tau_{h}(f)(x)=f\left(h^{-1} \cdot x\right)$ for $h \in H$ and $x \in X$ the induced action on $\mathrm{C}_{0}(X)$.

A $\mathrm{C}_{0}(X)$-algebra $A$ with $\theta: \mathrm{C}_{0}(X) \hookrightarrow Z \mathcal{M}(A)$ and an $H$-action $\alpha: H \curvearrowright A$ is called an $H-\mathrm{C}_{0}(X)$-algebra if the actions are compatible in the following sense

$$
\alpha_{h}(\theta(\varphi) a)=\theta\left(\tau_{h}(\varphi)\right) \alpha_{h}(a) \text { for all } h \in H, \varphi \in \mathrm{C}_{0}(X) \text { and } a \in A \text {. }
$$

Recall that the spectrum $\widehat{A}$ of a $\mathrm{C}^{*}$-algebra $A$ is the set of unitary equivalence classes of irreducible representations $\pi: A \rightarrow \mathbb{B}(\mathcal{H})$. It becomes a topological space when pulling back the Jacobson topology on $\operatorname{Prim}(A)$ to $\widehat{A}$ using the map $[\pi] \mapsto$ $\operatorname{ker}(\pi)$. If $H \curvearrowright A$ is a strongly continuous action, there is a continuous action $H \curvearrowright \widehat{A}$ given by $(x \cdot \pi)(a)=\pi\left(\alpha_{x^{-1}}(a)\right)$ for $[\pi] \in \widehat{A}, x \in H$ and $a \in A$.

Let $(A, \mathcal{R})$ be a continuously square-integrable $H$-C ${ }^{*}$-algebra. Every nondegenerate representation $\pi$ of $A$ can be extended to $\mathcal{M}(A)$. Denote its restriction to $\operatorname{Fix}^{H}(A, \mathcal{R})$ by $\tilde{\pi}$.

In the commutative case, this procedure allows to completely describe the representation theory of the generalized fixed point algebra. By Gelfand duality, the spectrum of the commutative $\mathrm{C}^{*}$-algebra $\mathrm{C}_{0}(X)$ is $X$. If $H \curvearrowright X$ is a proper action and $(A, \mathcal{R})=\left(\mathrm{C}_{0}(X), \overline{\mathrm{C}_{c}(X)}\right)$, the map $\pi \mapsto \tilde{\pi}$ induces a homeomorphism between $H \backslash \widehat{A}=H \backslash X$ and $\operatorname{Fix}^{H}(A, \mathcal{R})$.

This generalizes to $H$ - $\mathrm{C}_{0}(X)$-algebras as follows. We summarize some results concerning $H-\mathrm{C}_{0}(X)$-algebras proved in Mey01, aHRW00, Rie04, aHRW02 .

Proposition 2.28. Let $H \curvearrowright X$ be a proper action on a locally compact Hausdorff space $X$ and $A$ an $H-\mathrm{C}_{0}(X)$-algebra with $\theta: \mathrm{C}_{0}(X) \rightarrow Z \mathcal{M}(A)$.

(i) The subset $\mathcal{R}:=\overline{\theta\left(\mathrm{C}_{c}(X)\right) A}$ is dense, relatively continuous and complete. Moreover, it is the unique such subset. 
(ii) If the action of $H$ on $X$ is free, the map $\pi \mapsto \widetilde{\pi}$ induces a homeomorphism $H \backslash \widehat{A} \rightarrow \operatorname{Fix}^{H}(A, \mathcal{R})$ and $(A, \mathcal{R})$ is saturated.

Proof. An $H-\mathrm{C}_{0}(X)$-algebra where the action $H \curvearrowright X$ is proper is spectrally proper as argued in Mey01, Sec. 9]. The $\mathcal{R}$ above is the unique, dense, relatively continuous and complete subset constructed in Mey01, 9.4]. The first claim in (ii) is proved in aHRW00 3.9], the second in the preprint version of [Rie04 and in aHRW02, 4.1].

The following result applies to trivial continuous fields $\mathrm{C}_{0}(X, A)$, where the action is taking place on the base space $X$.

Lemma 2.29 ( Rie90, 2.6], [RW85, 3.2]). Let $H \curvearrowright X$ be a proper action and A a $\mathrm{C}^{*}$-algebra. Let $H$ act on $\mathrm{C}_{0}(X, A)$ by $\left(\tau_{h} f\right)(x)=f\left(h^{-1} \cdot x\right)$ for $h \in H$, $f \in \mathrm{C}_{0}(X, A)$ and $x \in X$. There is an isomorphism

$$
\begin{gathered}
\left.\Psi: \operatorname{Fix}^{H}\left(\mathrm{C}_{0}(X, A), \overline{\mathrm{C}_{c}(X, A}\right)\right) \rightarrow \mathrm{C}_{0}(H \backslash X, A), \\
\Psi(|f\rangle\rangle\langle\langle g|)(H x)=\int_{H}\left(f^{*} \cdot g\right)\left(h^{-1} \cdot x\right) \mathrm{d} h \quad \text { for } H x \in H \backslash X \text { and } f, g \in \mathrm{C}_{c}(H, A) .
\end{gathered}
$$

In particular, for $A=\mathbb{C}$ the generalized fixed point algebra is isomorphic to $\mathrm{C}_{0}(H \backslash X)$ as claimed before.

In contrast to the situation above, consider now what happens if the $H$-action only takes place in the fibres of a field of $\mathrm{C}^{*}$-algebras.

TheOREM 2.30 ( $[\mathbf{R i e 9 0}, 3.2])$. Let $A$ be a continuous field of $\mathrm{C}^{*}$-algebras over $X$ with fibre projections $p_{x}: A \rightarrow A_{x}$ for $x \in X$. Suppose that $(A, \mathcal{R})$ is a continuously square-integrable $H-\mathrm{C}^{*}$-algebra such that $\operatorname{ker}\left(p_{x}\right)$ is $H$-invariant for all $x \in X$. Furthermore, assume that $H$ is a $\sigma$-compact, exact group. Then $\operatorname{Fix}^{H}(A, \mathcal{R})$ is a continuous field of $\mathrm{C}^{*}$-algebras over $X$ with fibre projections

$$
\tilde{p}_{x}: \operatorname{Fix}^{H}(A, \mathcal{R}) \rightarrow \operatorname{Fix}^{H}\left(A_{x}, \overline{p_{x}(\mathcal{R})}\right) .
$$

Remark 2.31. The above theorem in Rie90 requires that the field $A$ is Hilbert continuous and that $\mathrm{C}_{\mathrm{r}}^{*}\left(H, A_{x}\right)=\mathrm{C}^{*}\left(\bar{H}, A_{x}\right)$ for all $x \in M$. These assumptions are only needed to show that $\mathrm{C}_{\mathrm{r}}^{*}(H, A)$ defines a continuous field of $\mathrm{C}^{*}$-algebras over $X$ with fibres $\mathrm{C}_{\mathrm{r}}^{*}\left(H, A_{x}\right)$. But this is true for any $\sigma$-compact, exact group $H$ by KW99, 4.2].

Now we study what can be said about saturatedness in this case.

Lemma 2.32. Let $A$ be an upper semi-continuous field of $\mathrm{C}^{*}$-algebras over $X$ with fibre projections $p_{x}: A \rightarrow A_{x}$. If $I \unlhd A$ is a proper ideal, then there is $x \in X$ such that $p_{x}(I) \triangleleft A_{x}$ is a proper ideal.

Proof. By Lee's Theorem (see [Lee76 or Nil96, 3.3]) there is a continuous $\operatorname{map} \psi: \operatorname{Prim}(A) \rightarrow X$ satisfying

$$
\psi(P)=x \Leftrightarrow P \subseteq K_{x}=\left\{a \in A \mid p_{x}(a)=0\right\}
$$

and $A_{x} \cong A / K_{x}$ for all $x \in X$. As $I$ can be written as the intersection of primitive ideals, it follows that there is a primitive ideal $P \in \operatorname{Prim}(A)$ with $I \subseteq P \subsetneq A$. Let $x=\psi(P)$. The homeomorphism $\left\{Q \in \operatorname{Prim}(A) \mid K_{x} \subseteq P\right\} \rightarrow \operatorname{Prim}\left(A / K_{x}\right)=$ $\operatorname{Prim}\left(A_{x}\right)$ maps $P$ to $p_{x}(P)$. Then $p_{x}(I) \subseteq p_{x}(P) \subseteq A_{x}$, and $p_{x}(P) \neq A_{x}$ as otherwise $p_{x}(P)$ would correspond to $A$ under this homeomorphism.

Corollary 2.33. In the situation of Theorem 2.30, $(A, \mathcal{R})$ is saturated if and only if $\left(A_{x}, \overline{p_{x}(\mathcal{R})}\right)$ is saturated for all $x \in X$. 
Proof. Suppose first that $(A, \mathcal{R})$ is saturated. By Corollary $\left.2.22\left(A_{x}, \overline{p_{x}(\mathcal{R}}\right)\right)$ is saturated for all $x \in X$.

Assume now that all $\left(A_{x}, \overline{p_{x}(\mathcal{R})}\right)$ are saturated. By assumption on $H, \mathrm{C}_{\mathrm{r}}^{*}(H, A)$ is a continuous field of $\mathrm{C}^{*}$-algebras over $X$ with fibre projections $\left(p_{x}\right)_{*}: \mathrm{C}_{\mathrm{r}}^{*}(H, A) \rightarrow$ $\mathrm{C}_{\mathrm{r}}^{*}\left(H, A_{x}\right)$ for $x \in X$. Because

$$
\left(p_{x}\right)_{*}(\langle\langle a \mid b\rangle\rangle)=\left\langle\left\langle p_{x}(a) \mid p_{x}(b)\right\rangle\right\rangle \quad \text { for } a, b \in \mathcal{R},
$$

it follows that $\left(p_{x}\right)_{*}\left(J^{H}(A, \mathcal{R})\right)=\mathrm{C}_{\mathrm{r}}^{*}\left(H, A_{x}\right)$ for all $x \in X$. Now Lemma 2.32 implies that $J^{H}(A, \mathcal{R})=\mathrm{C}_{\mathrm{r}}^{*}(H, A)$. 


\section{CHAPTER 3}

\section{Graded and homogeneous Lie groups}

In this chapter, we discuss graded and homogeneous Lie groups. These are Lie groups which are equipped with a dilation action of $\mathbb{R}_{>0}$ and, therefore, allow to define homogeneity with respect to the dilations. Detailed studies of these groups can be found in FS82 or FR16.

Definition 3.1. A graded Lie group is a simply connected Lie group whose Lie algebra $\mathfrak{g}$ admits a grading

$$
\mathfrak{g}=\bigoplus_{j=1}^{\infty} \mathfrak{g}_{\mathfrak{j}} \quad \text { with only finitely many } \mathfrak{g}_{j} \neq\{0\}
$$

which is compatible with the Lie bracket, that is, $\left[\mathfrak{g}_{j}, \mathfrak{g}_{k}\right] \subseteq \mathfrak{g}_{j+k}$ for all $j, k \in \mathbb{N}$. The graded Lie group is of step $r$ if $\mathfrak{g}_{r} \neq 0$ and $\mathfrak{g}_{j}=0$ for all $j>r$.

Note that the condition on the bracket forces the Lie algebra to be nilpotent. Consequently, also the Lie group is nilpotent.

Example 3.2. The simplest example of a non-Abelian graded Lie group is the $(2 n+1)$-dimensional Heisenberg group $\mathfrak{H}_{n}$. Its Lie algebra $\mathfrak{h}$ is generated by $\left\{X_{1}, \ldots, X_{n}, Y_{1}, \ldots, Y_{n}, Z\right\}$ satisfying $\left[X_{i}, Y_{i}\right]=Z$ and $\left[X_{i}, Z\right]=\left[Y_{i}, Z\right]=0$ for $i=1, \ldots, n$. Hence, $\mathfrak{h}_{1}=\operatorname{span}\left\{X_{1}, \ldots, X_{n}, Y_{1}, \ldots, Y_{n}\right\}, \mathfrak{h}_{2}=\operatorname{span}\{Z\}$ and $\mathfrak{h}_{j}=0$ for $j>2$ defines a grading of step 2 on $\mathfrak{h}$.

Example 3.3. For $n \geq 2$, the group of unitriangular $(n \times n)$-matrices is a graded Lie group of step $n-1$. The strictly upper triangular matrices $\mathfrak{n}$ are its Lie algebra. Let $E_{k l}$ be the matrix with entry 1 at $(k, l)$ and 0 otherwise. Then a grading is defined by $\mathfrak{n}_{j}=\operatorname{span}\left\{E_{k l} \mid l-k=j\right\}$ for $j=1, \ldots, n-1$ and $\mathfrak{n}_{j}=0$ for $j \geq n$.

Graded Lie algebras can be equipped with an action of $\mathbb{R}_{>0}$. Set $\lambda \cdot X=\lambda^{j} X$ for $X \in \mathfrak{g}_{j}$ and $\lambda>0$. This extends to an $\mathbb{R}_{>0}$-action by Lie algebra automorphisms.

Instead of starting with a grading, one can consider certain $\mathbb{R}_{>0}$-actions on Lie algebras. This yields the slightly more general class of homogeneous Lie groups.

Definition 3.4. For a Lie algebra $\mathfrak{g}$ and a diagonalizable, linear map $A: \mathfrak{g} \rightarrow \mathfrak{g}$ with positive eigenvalues $q_{1} \leq q_{2} \leq \ldots \leq q_{n}$, set $D_{\lambda}:=\operatorname{Exp}(A \ln (\lambda))$. Here, $\operatorname{Exp}$ denotes the matrix exponential. If all $D_{\lambda}$ are Lie algebra homomorphisms, $\left\{D_{\lambda}\right\}_{\lambda>0}$ is called a family of dilations.

A homogeneous Lie group is a simply connected Lie group $G$ whose Lie algebra $\mathfrak{g}$ is equipped with a family of dilations $\left\{D_{\lambda}\right\}_{\lambda>0}$. The eigenvalues $\left\{q_{1}, \ldots, q_{n}\right\}$ are called weights.

Folland and Stein assume in FS82 that $q_{1}=1$. This can be achieved by scaling appropriately. We shall also assume this in the following. In particular, all weights satisfy $q_{j} \geq 1$.

We fix a basis of eigenvectors $\left\{X_{1}, \ldots, X_{n}\right\}$ corresponding to the weights. Then $D_{\lambda}\left(X_{j}\right)=\lambda^{q_{j}} X_{j}$ holds for $1 \leq j \leq n$. 
Example 3.5. A Lie algebra may admit different dilations. For example, let $\left\{X_{1}, \ldots, X_{n}\right\}$ denote a basis for the Lie algebra of the Abelian group $G=\mathbb{R}^{n}$. Then for all $\left(q_{1}, \ldots, q_{n}\right) \in \mathbb{R}_{>0}^{n}$ there is a dilation defined by $D_{\lambda} X_{j}=\lambda^{q_{j}} X_{j}$. The standard dilation on $\mathbb{R}^{n}$ is given by scalar multiplication, that is, $q_{j}=1$ for all $j=1, \ldots, n$.

Lemma 3.6 ([FS82 1.3]). Homogeneous Lie groups are nilpotent.

Proof. Let $G$ be a homogeneous Lie group with dilations $D_{\lambda}$ and weights $1=$ $q_{1} \leq q_{2} \leq \ldots \leq q_{n}$. Suppose $X, Y$ are eigenvectors corresponding to the eigenvalues $q_{i}, q_{j}>0$ of $A$, respectively. Then $D_{\lambda}[X, Y]=\left[D_{\lambda}(X), D_{\lambda}(Y)\right]=\lambda^{q_{i}+q_{j}}[X, Y]$. Therefore, either $[X, Y]=0$ holds or $[X, Y]$ is an eigenvector of $A$ corresponding to the eigenvalue $q_{i}+q_{j}$. Using that $A$ has only finitely many eigenvalues, one can deduce that $\mathfrak{g}$ - and therefore $G$ - is nilpotent.

Consequently, the exponential map exp: $\mathfrak{g} \rightarrow G$ is a diffeomorphism. In the following, we often identify $\left(x_{1}, \ldots, x_{n}\right) \in \mathbb{R}^{n}$ with its image $\exp \left(x_{1} X_{1}+\cdots+\right.$ $\left.x_{n} X_{n}\right) \in G$ under this global coordinate chart. In particular, $0 \in G$ denotes the neutral element in a homogeneous Lie group and $x^{-1}=-x$ is the inverse. Moreover, the multiplication in $G$ is determined by the Lie bracket on $\mathfrak{g}$ by the Baker-Campbell-Hausdorff Formula (see, for example, [CG90, 1.2.1]).

Proposition 3.7 (Baker-Campbell-Hausdorff Formula). Let $G$ be a nilpotent Lie group and $X, Y \in \mathfrak{g}$. Then there is a unique $Z \in \mathfrak{g}$ be such that $\exp X \cdot \exp Y=$ $\exp Z$. It is given by

$$
\begin{array}{r}
Z=X+Y+\frac{1}{2}[X, Y]+\frac{1}{12}([X,[X, Y]]-[Y,[X, Y]]) \\
-\frac{1}{48}([Y,[X,[X, Y]]]-[X,[Y,[X, Y]]]) \\
+(\text { commutators of order } \geq 4) .
\end{array}
$$

As the Lie algebra is nilpotent only finitely many commutators up to a certain order appear in the formula above.

Because $D_{\lambda} \circ D_{\mu}=D_{\lambda \mu}$ for $\lambda, \mu>0$, the dilations define an action $D: \mathbb{R}_{>0} \curvearrowright \mathfrak{g}$ by Lie algebra automorphisms. Denote by $\delta: \mathbb{R}_{>0} \curvearrowright G$ the corresponding action by Lie group automorphisms. We shall also use the notation $\delta_{\lambda}(x)=\lambda \cdot x$ for $x \in G$ and $\lambda>0$.

Remark 3.8. If all weights of a homogeneous Lie group are rational numbers, it is a (scaled) graded Lie group (see [FR16, 3.1.9]). Hence, one can construct examples of homogeneous Lie groups that are not scaled graded Lie groups as in FR16 3.1.11]. Also note that there are nilpotent Lie groups that do not admit a family of dilations (see Dye70.

\subsection{Analysis on homogeneous Lie groups}

In this section we recall some definitions like homogeneous quasi-norms that proved to be useful to do analysis on homogeneous groups. The dilations induce new notions of homogeneity for functions and of the order for differential operators. We also consider Schwartz functions and (tempered) distributions on homogeneous groups.

Definition 3.9. The homogeneous dimension of a homogeneous Lie group $G$ with weights $1=q_{1} \leq q_{2} \leq \ldots \leq q_{n}$ is defined as $Q=q_{1}+q_{2}+\ldots+q_{n}$. A function $f$ on $G \backslash\{0\}$ is called $w$-homogeneous for $w \in \mathbb{C}$ if $f\left(\delta_{\lambda}(x)\right)=\lambda^{w} f(x)$ for all $x \neq 0$.

Lemma 3.10. Let $G$ be a homogeneous Lie group of homogeneous dimension $Q$. The pullback of the Lebesgue measure under the exponential map defines a Haar 
measure on $G$. The group $G$ is unimodular and the Haar measure is $Q$-homogeneous, that is,

$$
\int_{G} f\left(\delta_{\lambda}(x)\right) \mathrm{d} x=\lambda^{-Q} \int_{G} f(x) \mathrm{d} x
$$

for each $\lambda>0$ and $f \in L^{1}(G)$.

For simply connected nilpotent Lie groups the pullback of the Lebesgue measure defines a left and right Haar measure [FS82 1.2]. The $Q$-homogeneity follows from the behaviour of the Lebesgue measure under scaling.

Because the Euclidean norm does not behave well with respect to the dilations in general, we replace it by a homogeneous quasi-norm.

Definition 3.11 ([FR 3.1.33]). A homogeneous quasi-norm on a homogeneous Lie group $G$ is a continuous function $\|\cdot\|: G \rightarrow[0, \infty)$ with

(i) $\|x\|=0$ if and only if $x=0$,

(ii) $\|-x\|=\|x\|$ for all $x \in G$,

(iii) $\left\|\delta_{\lambda}(x)\right\|=\lambda\|x\|$ for all $x \in G$ and $\lambda \in \mathbb{R}_{>0}$.

In the following, we fix a homogeneous quasi-norm on $G$, for instance,

$$
\|x\|:=\sum_{j=1}^{n}\left|x_{j}\right|^{1 / q_{j}} \quad \text { for } x \in G .
$$

In fact, by [FR16, 3.1.35] all homogeneous quasi-norms on a given homogeneous Lie group are equivalent. There is an analogue of the triangle inequality for a homogeneous quasi-norm:

Lemma 3.12 ([FS82 1.8, 1.10]). Let $G$ be a homogeneous Lie group. There is a constant $\gamma \geq 1$ such that $\|x y\| \leq \gamma(\|x\|+\|y\|)$ for all $x, y \in G$.

For $R>0$ we define $R$-balls around $x \in G$ with respect to the quasi-norm by

$$
B(x, R)=\left\{y \in G \mid\left\|x y^{-1}\right\|<R\right\} .
$$

Using the dilations and the continuity of the quasi-norm one checks that the closure of $B(x, R)$ in the Euclidean topology is $\left\{y \in G \mid\left\|x y^{-1}\right\| \leq R\right\}$. Furthermore, the $B(x, R)$ are bounded as the Euclidean 1-norm $\|\cdot\|_{1}$ satisfies $\|x\|_{1} \leq n+\|x\|^{q_{n}}$ for $x \in G$ and the quasi-norm in (6). Hence, closed balls with respect to a homogeneous quasi-norm are compact and, in particular, have finite Haar measure.

Remark 3.13. Let $G$ be a graded Lie group and let $q$ be a common multiple of the weights $q_{1}, \ldots, q_{n} \in \mathbb{N}$. For the Euclidean norm $\|\cdot\|_{2}$, we have that $x \mapsto\|x\|_{2}^{2}=$ $\sum_{j=1}^{n} x_{j}^{2}$ is smooth. As this is not necessarily true for homogeneous quasi-norms, it is sometimes convenient to use

$$
\Phi(x):=\sum_{j=1}^{n} x_{j}^{2 q / q_{j}}
$$

instead. It is a smooth $(2 q)$-homogeneous function $G \rightarrow[0, \infty)$. Moreover, one can estimate $\|x\| \leq \Phi(x) \leq n\|x\|^{2 q}$ for all $x \in G$ and the homogeneous quasi-norm above.

We will use the following integrability criterion for functions on a homogeneous Lie group later on.

Lemma 3.14 ([FS82, 1.17]). Let $a \in \mathbb{R}$ and let $f$ be a measurable function on a homogeneous Lie group $G$ of homogeneous dimension $Q$. Suppose $|f(x)|=$ $O\left(\|x\|^{a-Q}\right)$. If $a>0$ then $f$ is integrable near 0 . If $a<0$, then $f$ is integrable near infinity. 
Definition 3.15. For a multi-index $\alpha \in \mathbb{N}_{0}^{n}$ its homogeneous degree is defined as $[\alpha]:=\alpha_{1} q_{1}+\cdots+\alpha_{n} q_{n}$, while its Euclidean degree is $|\alpha|:=\alpha_{1}+\cdots+\alpha_{n}$. A function $P$ on $G$ is called polynomial if $P \circ \exp$ is polynomial.

Example 3.16. The polynomials $x^{\alpha}$ for $\alpha \in \mathbb{N}_{0}^{n}$ are $[\alpha]$-homogeneous functions.

Using the Baker-Campbell-Hausdorff formula (5) and the homogeneity of the coordinate functions, the following is proved in FR16, 3.1.24]:

Proposition 3.17. For a homogeneous Lie group $G$ with weights $q_{1} \leq \ldots \leq q_{n}$ and a corresponding basis of eigenvectors $X_{1}, \ldots, X_{n} \in \mathfrak{g}$ there are constants $c_{j, \alpha, \beta}$ for $j=1, \ldots, n$ such that for all $x, y \in G$ with respect to this basis

$$
(x \cdot y)_{j}=x_{j}+y_{j}+\sum_{\substack{\alpha, \beta \in \mathbb{N}_{0}^{n} \backslash\{0\} \\[\alpha]+[\beta]=q_{j}}} c_{j, \alpha, \beta} x^{\alpha} y^{\beta} .
$$

The Poincaré-Birkhoff-Witt Theorem identifies the universal enveloping algebra $\mathcal{U}(\mathfrak{g})$ with the left-invariant, respectively right-invariant, differential operators on $G$.

Definition 3.18. For the basis of eigenvalues fixed above, define the left- and rightinvariant differential operators $X_{1}, \ldots, X_{n}$ and $Y_{1}, \ldots, Y_{n}$ by setting for $f \in \mathrm{C}^{1}(G)$

$$
\begin{aligned}
\left(X_{j} f\right)(x) & =\left.\frac{\mathrm{d}}{\mathrm{d} t} f\left(x \cdot \exp \left(t X_{j}\right)\right)\right|_{t=0}, \\
\left(Y_{j} f\right)(x) & =\left.\frac{\mathrm{d}}{\mathrm{d} t} f\left(\exp \left(t X_{j}\right) \cdot x\right)\right|_{t=0} .
\end{aligned}
$$

Left, respectively right-invariant, means that they commute with the left regular representation $\pi_{L}$, respectively right regular representation $\pi_{R}$, given by

$$
\begin{aligned}
& \left(\pi_{L}(x) f\right)(y)=f\left(x^{-1} y\right), \\
& \left(\pi_{R}(x) f\right)(y)=f(y x)
\end{aligned}
$$

for $f: G \rightarrow \mathbb{C}$ and $x, y \in G$. For a multi-index $\alpha \in \mathbb{N}_{0}^{n}$ set

$$
\begin{aligned}
X^{\alpha} & =X_{1}^{\alpha_{1}} X_{2}^{\alpha_{2}} \cdots X_{n}^{\alpha_{n}}, \\
Y^{\alpha} & =Y_{1}^{\alpha_{1}} Y_{2}^{\alpha_{2}} \cdots Y_{n}^{\alpha_{n}} .
\end{aligned}
$$

The operators $X^{\alpha}$ and $Y^{\alpha}$ for $\alpha \in \mathbb{N}_{0}^{n}$ form a basis for the left-, respectively right-invariant, differential operators on $G$. Let $\mathcal{U}(\mathfrak{g})$ be equipped with the $\mathbb{R}_{>0}$-action induced by the dilations on $\mathfrak{g}$. For a differential operator $P$ define

$$
\delta_{\lambda}^{*}(P) f(x):=P\left(f \circ \delta_{\lambda}\right)\left(\lambda^{-1} \cdot x\right) \text { for } \lambda>0 \text { and } x \in G .
$$

Assigning to an element of $\mathcal{U}(\mathfrak{g})$ its left-invariant or right-invariant differential operator is $\mathbb{R}_{>0}$-equivariant for these actions. We say that a differential operator $P$ is homogeneous of order $q$ if $\delta_{\lambda}^{*}(P)=\lambda^{q} P$. In particular, $X^{\alpha}$ and $Y^{\alpha}$ are homogeneous of order $[\alpha]$.

The triangular form of the group law allows to express $X_{j}$ and $Y_{j}$ in terms of the partial differential operators with respect to the coordinate functions as follows:

Proposition 3.19 ([FR16 3.1.28]). Let $G$ be a homogeneous Lie group with weights $q_{1} \leq \ldots \leq q_{n}$. For $j=1, \ldots, n$ and $k>j$ there are $\left(q_{k}-q_{j}\right)$-homogeneous polynomials $P_{j k}$ and $Q_{j k}$ such that the vector fields $X_{j}$ and $Y_{j}$ defined above can be 
written as

$$
\begin{aligned}
& X_{j}=\frac{\partial}{\partial x_{j}}+\sum_{q_{k}>q_{j}} P_{j k} \frac{\partial}{\partial x_{k}}=\frac{\partial}{\partial x_{j}}+\sum_{q_{k}>q_{j}} \frac{\partial}{\partial x_{k}} P_{j k}, \\
& Y_{j}=\frac{\partial}{\partial x_{j}}+\sum_{q_{k}>q_{j}} Q_{j k} \frac{\partial}{\partial x_{k}}=\frac{\partial}{\partial x_{j}}+\sum_{q_{k}>q_{j}} \frac{\partial}{\partial x_{k}} Q_{j k} .
\end{aligned}
$$

The polynomials $P_{j k}$ and $Q_{j k}$ only depend on $\left(x_{1}, \ldots, x_{k-1}\right)$ because otherwise they would be homogeneous of a higher order than $q_{k}-q_{j}$. Hence, they commute with the partial derivatives $\frac{\partial}{\partial x_{k}}$.

Identifying $G$ with $\mathbb{R}^{n}$ using the exponential map, one can consider the Schwartz space $\mathcal{S}(G)$. One can replace the partial derivatives in the usual definition of the Schwartz seminorms by the operators $X_{j}$ or $Y_{j}$ or vice versa because of Proposition 3.19 Furthermore, polynomials in $\|x\|_{2}$ for the Euclidean norm can be estimated by polynomials in $\|x\|$ for a homogeneous quasi-norm and the other way around. Therefore, one may use the following family of seminorms defined in $[$ FS82].

Definition 3.20. For a fixed homogeneous quasi-norm $\|\cdot\|$ and $N \in \mathbb{N}_{0}$ define

$$
\|f\|_{N}:=\sup _{[\alpha] \leq N, x \in G}(1+\|x\|)^{N}\left|\left(X^{\alpha} f\right)(x)\right| \text { for } f \in \mathrm{C}_{c}^{\infty}(G) .
$$

The Schwartz space $\mathcal{S}(G)$ is the completition of $\mathrm{C}_{c}^{\infty}(G)$ with respect to the seminorms $\|\cdot\|_{N}$ for $N \in \mathbb{N}$.

Lemma 3.21 ([FS82 1.46, 1.47]). The involution and convolution with respect to the group multiplication on $G$ given by

$$
\begin{aligned}
f^{*}(x) & =\overline{f\left(x^{-1}\right)}, \\
(f * g)(x) & =\int_{G} f(y) g\left(y^{-1} x\right) \mathrm{d} y
\end{aligned}
$$

define continuous maps $\mathcal{S}(G) \rightarrow \mathcal{S}(G)$ and $\mathcal{S}(G) \times \mathcal{S}(G) \rightarrow \mathcal{S}(G)$.

The following lemma shows how $X^{\alpha}$ and $Y^{\alpha}$ interact with the convolution.

Lemma 3.22. For $f \in \mathcal{S}(G)$, define $\tilde{f} \in \mathcal{S}(G)$ by $\tilde{f}(x)=f\left(x^{-1}\right)$. Then the leftand right-invariant differential operators are related by

$$
\widetilde{Y^{\alpha} f}=(-1)^{|\alpha|} X^{\alpha} \tilde{f} \quad \text { and } \quad \widetilde{X^{\alpha} f}=(-1)^{|\alpha|} Y^{\alpha} \tilde{f}
$$

for $f \in \mathcal{S}(G)$. Moreover, they behave as follows with respect to the convolution:

$$
X^{\alpha}(f * g)=f *\left(X^{\alpha} g\right), \quad Y^{\alpha}(f * g)=\left(Y^{\alpha} f\right) * g, \quad\left(X^{\alpha} f\right) * g=f *\left(Y^{\alpha} g\right)
$$

for $f, g \in \mathcal{S}(G)$.

We denote by $\mathcal{S}^{\prime}(G)$ the space of tempered distributions, the continuous dual of the Schwartz space. We recall some facts on distributions that are well-known for $G=\mathbb{R}^{n}$ (see for example Rud91]), and that carry over to homogeneous groups. First, the convolution of a tempered distribution and a Schwartz function is defined:

Lemma 3.23. There are well-defined, bilinear, separately continuous maps

$$
\begin{array}{lll}
*: \mathcal{S}^{\prime}(G) \times \mathcal{S}(G) \rightarrow \mathcal{S}^{\prime}(G), & & \langle u * f, g\rangle:=\langle u, g * \tilde{f}\rangle, \\
*: \mathcal{S}(G) \times \mathcal{S}^{\prime}(G) \rightarrow \mathcal{S}^{\prime}(G), & & \langle f * u, g\rangle:=\langle u, \tilde{f} * g\rangle,
\end{array}
$$

for $u \in \mathcal{S}^{\prime}(G)$ and $f, g \in \mathcal{S}(G)$. They satisfy the following associativity properties $(f * g) * u=f *(g * u), \quad(u * f) * g=u *(f * g) \quad$ and $\quad(f * u) * g=f *(u * g)$ for $u \in \mathcal{S}^{\prime}(G)$ and $f, g \in \mathcal{S}(G)$. 
Moreover, the action of $X^{\alpha} \in \mathcal{U}(\mathfrak{g})$ for $\alpha \in \mathbb{N}$ as a left-invariant or rightinvariant operators on $\mathcal{S}(G)$ can be extended to $\mathcal{S}^{\prime}(G)$ by setting for $u \in \mathcal{S}^{\prime}(G)$ and $f \in \mathcal{S}(G)$

$$
\begin{aligned}
\left\langle X^{\alpha} u, f\right\rangle & :=(-1)^{|\alpha|}\left\langle u, X^{\alpha} f\right\rangle, \\
\left\langle Y^{\alpha} u, f\right\rangle & :=(-1)^{|\alpha|}\left\langle u, Y^{\alpha} f\right\rangle .
\end{aligned}
$$

Let $\mathcal{E}^{\prime}(G)$ denote the continuous dual of $\mathcal{E}(G)=\mathrm{C}^{\infty}(G)$. For the delta distribution $u=\delta \in \mathcal{E}^{\prime}(G)$ defined by $\langle\delta, f\rangle=f(0)$, set $X^{\alpha}:=X^{\alpha} \delta=Y^{\alpha} \delta \in \mathcal{E}^{\prime}(G)$, slightly abusing notation.

Note that for $u \in \mathcal{S}^{\prime}(G)$ and $f \in \mathcal{S}(G)$ the convolution $u * f$ belongs to $\mathrm{C}^{\infty}(G)$. Therefore, the convolution of a compactly supported distribution $v \in \mathcal{E}^{\prime}(G)$ and $u \in \mathcal{S}^{\prime}(G)$ is well-defined by setting

$$
\langle v * u, f\rangle:=\langle v, f * \tilde{u}\rangle .
$$

Here, $\tilde{u} \in \mathcal{S}^{\prime}(G)$ is defined by $\langle\tilde{u}, f\rangle=\langle u, \tilde{f}\rangle$ for $f \in \mathcal{S}(G)$.

Lemma 3.24. Let $X^{\alpha} \in \mathcal{U}(\mathfrak{g}), u \in \mathcal{S}^{\prime}(G)$ and $f \in \mathcal{S}(G)$. Then the following holds:

(i) $X^{\alpha} * u=Y^{\alpha} u$ and $u * X^{\alpha}=X^{\alpha} u$,

(ii) $f *\left(X^{\alpha} * u\right)=\left(f * X^{\alpha}\right) * u$.

Proof. For (i) note first that for $f \in \mathcal{S}(G)$ and $u \in \mathcal{S}(G), X^{\alpha}(f * u)=$ $f *\left(X^{\alpha} u\right)$ and $X^{\alpha} \tilde{u}=(-1)^{|\alpha|} \widetilde{Y^{\alpha} u}$. These follow from the corresponding properties on $\mathcal{S}(G)$. Hence, for all $f \in \mathcal{S}(G)$

$$
\left\langle X^{\alpha} * u, f\right\rangle=(-1)^{|\alpha|}\left\langle\delta, X^{\alpha}(f * \tilde{u})\right\rangle=\left\langle\delta, f * \widetilde{Y^{\alpha}} u\right\rangle=\left\langle Y^{\alpha} u, f\right\rangle
$$

holds. The second claim is proved analogously. For (ii) note that the left hand side is well-defined as $X^{\alpha} * u \in \mathcal{S}^{\prime}(G)$ by (i) It remains to show $f *\left(Y^{\alpha} u\right)=\left(X^{\alpha} f\right) * u$. One computes for $g \in \mathcal{S}(G)$

$$
\begin{aligned}
\left\langle f *\left(Y^{\alpha} u\right), g\right\rangle & =\left\langle Y^{\alpha} u, \tilde{f} * g\right\rangle=(-1)^{|\alpha|}\left\langle u, Y^{\alpha}(\tilde{f} * g)\right\rangle=(-1)^{|\alpha|}\left\langle u,\left(Y^{\alpha} \tilde{f}\right) * g\right\rangle \\
& =\left\langle u, \widetilde{X^{\alpha} f} * g\right\rangle=\left\langle\left(X^{\alpha} f\right) * u, g\right\rangle .
\end{aligned}
$$

Lemma 3.25. Let $\phi: H \rightarrow G$ be an injective, $\mathbb{R}_{>0}$-equivariant, smooth homomorphism between homogeneous Lie groups $H, G$. It induces continuous linear maps

$$
\begin{aligned}
\phi^{*}: \mathcal{S}(G) & \rightarrow \mathcal{S}(H), & f & \mapsto f \circ \phi, \\
\phi_{*}: \mathcal{S}^{\prime}(H) & \rightarrow \mathcal{S}^{\prime}(G), & \left\langle\phi_{*}(u), f\right\rangle & =\left\langle u, \phi^{*}(f)\right\rangle .
\end{aligned}
$$

They satisfy

(i) $\phi_{*}(\delta)=\delta$,

(ii) $\phi_{*}(\tilde{u})=\widetilde{\phi_{*}(u)}$ for $u \in \mathcal{S}^{\prime}(H)$,

(iii) $\phi_{*}(u) \in \mathcal{E}^{\prime}(G)$ for $u \in \mathcal{E}^{\prime}(H)$,

(iv) $\phi_{*}(u * v)=\phi_{*}(u) * \phi_{*}(v)$ for all $u \in \mathcal{E}^{\prime}(H)$ and $v \in \mathcal{S}^{\prime}(H)$.

Proof. Pick homogeneous quasi-norms on $H$ and $G$. As $x \mapsto\|\phi(x)\|$ is continuous, it attains a minimum $C$ on the anisotropic sphere $S=\{x \in H \mid\|x\|=1\}$. Because $\phi$ is an injective homomorphism, $C>0$ must hold. For $x \in H$ arbitrary, set $\lambda=\|x\|$. As $\phi$ is equivariant for the dilations,

$$
\|\phi(x)\|=\left\|\lambda \cdot \phi\left(\lambda^{-1} \cdot x\right)\right\|=\lambda\left\|\phi\left(\lambda^{-1} \cdot x\right)\right\| \geq C\|x\| .
$$

For $X \in \mathfrak{h}, X(f \circ \phi)=(\mathrm{d} \phi(X)(f)) \circ \phi$ holds. Therefore, one obtains constants $C_{N}$ for $N \in \mathbb{N}$ such that $\left\|\phi^{*}(f)\right\|_{N} \leq C_{N}\|f\|_{N}$ for all $f \in \mathcal{S}(G)$. This norm estimate implies that for $f_{k} \rightarrow f$ in $\mathcal{S}(\bar{G})$ also $\phi^{*}\left(f_{k}\right) \rightarrow \phi^{*}(f)$, which means that $\phi^{*}$ is continuous. 
For $u \in \mathcal{S}^{\prime}(H), \phi_{*}(u)$ is a linear functional. It is continuous as $f_{j} \rightarrow 0$ in $\mathcal{S}(G)$ implies that $\phi^{*}\left(f_{j}\right) \rightarrow 0$, so that $\left\langle\phi_{*}(u), f_{j}\right\rangle=\left\langle u, \phi^{*}\left(f_{i}\right)\right\rangle \rightarrow 0$. Moreover, $\phi_{*}$ is linear and continuous, because if $u_{j} \rightarrow u$ in $\mathcal{S}^{\prime}(H)$, then for $f \in \mathcal{S}(G)$

$$
\left\langle\phi_{*}\left(u_{j}\right), f\right\rangle=\left\langle u_{j}, \phi^{*}(f)\right\rangle \rightarrow\left\langle u, \phi^{*}(f)\right\rangle=\left\langle\phi_{*}(u), f\right\rangle .
$$

As $\phi(0)=0$, (i) is clear. Property (ii) follows as $\phi$ is a homomorphism. For (iii) we need that $\phi$ is proper. This follows from continuity of $\phi$ and 10 . In particular, the right hand side of (iv) is well-defined. Let $u \in \mathcal{E}^{\prime}(H), v \in \mathcal{S}^{\prime}(H)$ and $f \in \mathcal{S}(G)$. Then

$$
\begin{aligned}
\left\langle\phi_{*}(u * v), f\right\rangle & =\langle u,(f \circ \phi) * \tilde{v}\rangle, \\
\left\langle\phi_{*}(u) * \phi_{*}(v), f\right\rangle & =\left\langle u,\left(f * \widetilde{\phi_{*} v}\right) \circ \phi\right\rangle .
\end{aligned}
$$

Using (ii), it remains to show

$$
(f \circ \phi) * v=\left(f * \phi_{*}(v)\right) \circ \phi \quad \text { for all } v \in \mathcal{S}(H) .
$$

This follows from

$$
\pi_{R}\left(x^{-1}\right) \widetilde{f \circ \phi}=\left(\pi_{R}\left(\phi(x)^{-1}\right) \tilde{f}\right) \circ \phi
$$

for all $f \in \mathcal{S}(G)$, which uses again that $\phi$ is a homomorphism.

\subsection{Representation theory and Kirillov's orbit method}

Now, we recall some facts about the representation theory of nilpotent Lie groups and their group $\mathrm{C}^{*}$-algebras. For homogeneous Lie groups, the dilations induce actions on the respective spaces of representations.

The vector space of continuous compactly supported functions $\mathrm{C}_{c}(G)$ becomes a *-algebra when equipped with the same involution and convolution as in Lemma 3.21

Definition 3.26. For a homogeneous Lie group $G$ denote by $\widehat{G}$ the set of equivalence classes of irreducible, unitary representations $\pi: G \rightarrow \mathcal{U}\left(\mathcal{H}_{\pi}\right)$. For such a representation $\pi$ and $f \in \mathrm{C}_{c}(G)$ define the operator

$$
\widehat{\pi}(f)=\int_{G} f(x) \pi(x) \mathrm{d} x \in \mathbb{B}\left(\mathcal{H}_{\pi}\right) \quad \text { for } f \in \mathrm{C}_{c}(G) .
$$

This defines a ${ }^{*}$-representation $\widehat{\pi}: \mathrm{C}_{c}(G) \rightarrow \mathbb{B}\left(\mathcal{H}_{\pi}\right)$. The full group $\mathrm{C}^{*}$-algebra $\mathrm{C}^{*}(G)$ is defined as the closure of $\mathrm{C}_{c}(G)$ with respect to $\|f\|=\sup _{\pi \in \widehat{G}}\|\widehat{\pi}(f)\|$. It contains the Schwartz space $\mathcal{S}(G)$.

By Dix59 homogeneous Lie groups are liminal, so that all representations $\widehat{\pi}$ map onto the compact operators $\mathbb{K}\left(\mathcal{H}_{\pi}\right)$.

The homogeneous structure allows to define an $\mathbb{R}_{>0}$-action on $\widehat{G}$. For an irreducible, unitary representation $\pi$ set $(\lambda \cdot \pi)(x)=\pi\left(\delta_{\lambda^{-1}}(x)\right)$ for $\lambda>0$ and $x \in G$. It is easy to see that $\lambda \cdot \pi$ is again an irreducible, unitary representation and that the action is well-defined on the equivalence classes.

Furthermore, we define a strongly continuous action $\sigma: \mathbb{R}_{>0} \curvearrowright \mathrm{C}^{*}(G)$ by

$$
\sigma_{\lambda}(f)(x)=\lambda^{Q} f\left(\delta_{\lambda}(x)\right) \text { for } \lambda>0, f \in \mathrm{C}_{c}(G) \text { and } x \in G .
$$

It is not hard to check using the homogeneity of the Haar measure in Lemma 3.10 that each $\sigma_{\lambda}$ is a ${ }^{*}$-homomorphism and an isometry with respect to the $\mathrm{C}^{*}$-norm.

This action on $\mathrm{C}^{*}(G)$ induces in turn an action on the spectrum of $\mathrm{C}^{*}(G)$ by $(\lambda \cdot \rho)(f)=\rho\left(\sigma_{\lambda^{-1}}(f)\right)$ for a ${ }^{*}$-representation $\rho: \mathrm{C}^{*}(G) \rightarrow \mathbb{B}\left(\mathcal{H}_{\pi}\right)$. Again, it is well-defined on the equivalence classes of irreducible representations.

Proposition 3.27. For a homogeneous Lie group $G$ the map $\widehat{G} \rightarrow \widehat{\mathrm{C}^{*}(G)}$ induced by $\pi \mapsto \widehat{\pi}$ is an $\mathbb{R}_{>0}$-equivariant bijection. 
Proof. It is well-known that the map above is a bijection for each locally compact group $G$. The equivariance under the $\mathbb{R}_{>0}$-action follows because the Haar measure is $Q$-homogeneous, so that

$$
(\lambda \cdot \widehat{\pi})(f)=\int_{G}\left(\sigma_{\lambda^{-1}} f\right)(x) \pi(x) \mathrm{d} x=\int_{G} f(x) \pi\left(\delta_{\lambda^{-1}}(x)\right) \mathrm{d} x=\widehat{\lambda \cdot \pi}(f)
$$

for $\lambda>0$ and $f \in \mathrm{C}_{c}(G)$.

In particular, $\widehat{G}$ becomes a topological space with respect to the topology on $\widehat{\mathrm{C}^{*}(G)}$ under the bijection above. Kirillov's orbit method Kir62 allows to describe $\widehat{G}$ as the orbit space of the coadjoint action of $G$ on $\mathfrak{g}^{*}$, the dual of its Lie algebra.

Recall that the adjoint representation $\operatorname{Ad}: G \rightarrow \operatorname{Aut}(\mathfrak{g})$ is given by $\operatorname{Ad}(x)=$ $d\left(\alpha_{x}\right)_{0}: T_{0} G \rightarrow T_{0} G$, where $\alpha_{x}(y)=x y x^{-1}$ is given by conjugation. The coadjoint action is defined by

$$
\langle\operatorname{co}-\operatorname{Ad}(x) l, X\rangle:=\left\langle l, \operatorname{Ad}\left(x^{-1}\right) X\right\rangle
$$

for $l \in \mathfrak{g}^{*}, x \in G$ and $X \in \mathfrak{g}$. The corresponding infinitesimal representation co-ad of $\mathfrak{g}$ on $\mathfrak{g}^{*}$ is given by $\langle\operatorname{co}-\operatorname{ad}(X) l, Y\rangle=\langle l,[Y, X]\rangle$ for $l \in \mathfrak{g}^{*}$ and $X, Y \in \mathfrak{g}$.

The orbit space $G \backslash \mathfrak{g}^{*}$ is equipped with the quotient topology. For each $l \in \mathfrak{g}^{*}$, one can construct a unitary representation $\pi_{l}$ of $G$ as described in the following. Define a skew-symmetric bilinear form $b_{l}: \mathfrak{g} \times \mathfrak{g} \rightarrow \mathbb{R}$ by

$$
b_{l}(X, Y)=\langle l,[X, Y]\rangle \text { for } X, Y \in \mathfrak{g} .
$$

Denote by $\mathfrak{g}_{l}$ its radical. A subspace $V \subset \mathfrak{g}$ is isotropic with respect to $b_{l}$ if $b_{l}(X, Y)=0$ for all $X, Y \in V$. A maximal isotropic subspace has codimen$\operatorname{sion} \frac{1}{2} \operatorname{dim}\left(\mathfrak{g} / \mathfrak{g}_{l}\right)$. A polarizing subalgebra for $l$ is a subalgebra $\mathfrak{h}_{\mathfrak{l}} \subset \mathfrak{g}$ that is an isotropic subspace of codimension $\frac{1}{2} \operatorname{dim}\left(\mathfrak{g} / \mathfrak{g}_{l}\right)$. Such a polarizing subalgebra always exists (see CG90, 1.3.3, 1.3.5]).

The formula $\chi_{l}(\exp X)=e^{i\langle l, X\rangle}$ for $X \in \mathfrak{h}_{\mathfrak{l}}$ defines a one-dimensional representation of $H_{l}=\exp \left(\mathfrak{h}_{l}\right)$. It is multiplicative, because if $\exp X \cdot \exp Y=\exp Z$ for $X, Y \in \mathfrak{h}_{l}$, then $Z$ is given by the Baker-Campbell-Hausdorff formula (5) as $Z=X+Y+\frac{1}{2}[X, Y]+\frac{1}{12}([X,[X, Y]]-[Y,[X, Y]])+($ commutators of order $\geq 3)$, so that all higher terms lie in $\left[\mathfrak{h}_{l}, \mathfrak{h}_{l}\right] \subset \operatorname{ker} l$. Denote by $\pi_{l}=\operatorname{Ind}_{H_{l}}^{G} \chi_{l}$ the induced representation of $\chi_{l}$ from $H_{l}$ to $G$.

Let $\mathbb{R}_{>0}$ act on $\mathfrak{g}^{*}$ by $\langle\lambda \cdot l, X\rangle=\left\langle l, D_{\lambda^{-1}}(X)\right\rangle$ for $\lambda>0, l \in \mathfrak{g}^{*}$ and $X \in \mathfrak{g}$. This action descends to the orbit space of the coadjoint action as $D_{\lambda} \circ \operatorname{Ad}(x)=$ $\operatorname{Ad}(\lambda \cdot x) \circ D_{\lambda}$ for $\lambda>0$ and $x \in G$.

Lemma 3.28 ([CG90, 2.1.3]). Let $H$ be a subgroup of a locally compact group $G$ and let $\alpha$ be an automorphism of $G$ and $\pi$ a unitary representation of $H$. Then $\alpha^{-1}(H)$ is also a subgroup and

$$
\operatorname{Ind}_{\alpha^{-1}(H)}^{G}(\pi \circ \alpha) \simeq\left(\operatorname{Ind}_{H}^{G} \pi\right) \circ \alpha .
$$

Lemma 3.29. Kirillov's map $G \backslash \mathfrak{g}^{*} \rightarrow \widehat{G}$ induced by $l \mapsto \pi_{l}$ is an $\mathbb{R}_{>0}$-equivariant homeomorphism.

Proof. Kirillov proved in Kir62 that the map is well-defined. In particular, the equivalence class of $\pi_{l}$ does not depend on the choice of the polarizing subalgebra $\mathfrak{h}_{l}$. Two representations $\pi_{l_{1}}$ and $\pi_{l_{2}}$ are equivalent if and only if $l_{1}$ and $l_{2}$ lie in the same coadjoint orbit. Moreover, he proved that the map is continuous and onto. The continuity of the inverse map is due to Bro73. To see that the map is equivariant, note that $\chi_{\lambda \cdot l}=\chi_{l} \circ \delta_{\lambda^{-1}}$ and that $\delta_{\lambda}(H)$ is a polarizing subalgebra for $\lambda \cdot l$. Hence, Lemma 3.28 yields that $\pi_{\lambda \cdot l} \simeq \lambda \cdot \pi_{l}$. 
Note that all $l \in \mathfrak{g}^{*}$ that vanish on $[\mathfrak{g}, \mathfrak{g}]$ induce one-dimensional representations $\pi_{l}$. In particular, $l=0$ induces the trivial representation on $\mathbb{C}$. If the polarizing subalgebra is not all of $\mathfrak{g}$, the corresponding Hilbert space is infinitedimensional.

\subsection{Stratification of the representations}

The goal of this section is to use Kirillov theory and the coarse stratification by Pukánszky $\mathbf{P u k 6 7 ]}$ to find a sequence of increasing, open, $\mathbb{R}_{>0}$-invariant subsets

$$
\emptyset=V_{0} \subset V_{1} \subset V_{2} \subset \ldots \subset V_{m}=\widehat{G} \backslash\left\{\pi_{\text {triv }}\right\}
$$

such that all $\Lambda_{i}:=V_{i} \backslash V_{i-1}$ are Hausdorff and the $\mathbb{R}_{>0}$-action on each of these subsets is free and proper. This sequence will play an essential role in Chapter 10

Note that the following construction to find such a sequence of open subsets works for all simply connected nilpotent Lie groups. However, a dilation action is only defined for homogeneous Lie groups.

We start by describing Pukánszky's stratification of $\mathfrak{g}^{*}$. Recall that we fixed a basis $\left\{X_{1}, \ldots, X_{n}\right\}$ of $\mathfrak{g}$ such that $D_{\lambda}\left(X_{j}\right)=\lambda^{q_{j}} X_{j}$ for all $1 \leq j \leq n$. By the triangular form of the group law (7) all

$$
\mathfrak{k}_{i}=\operatorname{span}\left\{X_{i+1}, \ldots, X_{n}\right\} \quad \text { for } i=0, \ldots, n
$$

form an ideal in $\mathfrak{g}$. In particular, $\left\{X_{1}, \ldots, X_{n}\right\}$ is a strong Malcev basis of $G$ as defined in CG90 1.1.13], which is also called a Jordan-Hölder basis in Puk67. Note that they require $\operatorname{span}\left\{X_{1}, \ldots, X_{i}\right\}$ to be ideals. We stick to the reversed ordering of the basis as this is standard for homogeneous Lie groups.

Let $\left\{X_{1}^{*}, \ldots, X_{n}^{*}\right\}$ denote the corresponding dual basis of $\mathfrak{g}^{*}$ and let $\mathfrak{k}_{i}^{\perp}=$ $\operatorname{span}\left\{X_{1}^{*}, \ldots, X_{i}^{*}\right\}$ for $i=0, \ldots, n$. An element $l \in \mathfrak{g}^{*}$ is contained in $\mathfrak{k}_{i}^{\perp}$ if and only if $\left\langle l, \mathfrak{k}_{i}\right\rangle=0$. As the $\mathfrak{k}_{i}$ are ideals and are, therefore, invariant under the adjoint action, the $\mathfrak{k}_{i}^{\perp}$ are invariant under the coadjoint action. Hence $G$ acts on each $\mathfrak{g}^{*} / \mathfrak{k}_{i}^{\perp}$.

Write $p_{i}: \mathfrak{g}^{*} \rightarrow \mathfrak{g}^{*} / \mathfrak{k}_{i}^{\perp}$ for the projection. By CG90 3.1.4] the orbits $G \cdot p_{i}(l)$ of $p_{i}(l)$ under the coadjoint action are closed. Hence, they define submanifolds of $\mathfrak{g}^{*} / \mathfrak{k}_{i}^{\perp}$. Following CG90, make the following definition.

Definition 3.30. For $l \in \mathfrak{g}^{*}$ let $d(l)=\left(d_{0}(l), d_{1}(l), \ldots, d_{n-1}(l)\right)$ denote the sequence of orbit dimensions $d_{i}(l)=\operatorname{dim}\left(G \cdot p_{i}(l)\right)$.

The entries of $d(l)$ are decreasing. The corresponding stabilizer subgroups $G_{p_{i}(l)}$ form an increasing sequence

$$
G_{p_{0}(l)} \subseteq G_{p_{1}(l)} \subseteq \ldots \subseteq G_{p_{n-1}(l)} .
$$

The same is true for their Lie algebras $\mathfrak{g}_{p_{i}(l)}$. By [CG90, 3.1.1] they are given by

$$
\begin{aligned}
\mathfrak{g}_{p_{i}(l)} & =\left\{X \in \mathfrak{g} \mid \operatorname{co-ad}(X) l \in \mathfrak{k}_{i}^{\perp}\right\} \\
& =\left\{X \in \mathfrak{g} \mid\left\langle l,\left[X, X_{k}\right]\right\rangle=0 \text { for } k=i+1, \ldots, n\right\} .
\end{aligned}
$$

Example 3.31. The computation in CG90,3.1.11] of the coadjoint action on the 3-dimensional Heisenberg group $\mathbb{H}_{1}$ yields

$$
\operatorname{co-} \operatorname{Ad}(x, y, z) \alpha X^{*}+\beta Y^{*}+\gamma Z^{*}=(\alpha+y \gamma) X^{*}+(\beta-x \gamma) Y^{*}+\gamma Z^{*}
$$

for $(x, y, z) \in \mathbb{H}_{1}$ and $\alpha, \beta, \gamma \in \mathbb{R}$. This shows for $X_{1}=X, X_{2}=Y$ and $X_{3}=Z$ that

$$
\begin{aligned}
d\left(\alpha X^{*}+\beta Y^{*}+\gamma Z^{*}\right) & =(2,1,0) \quad \text { if } \gamma \neq 0, \\
d\left(\alpha X^{*}+\beta Y^{*}\right) & =(0,0,0) .
\end{aligned}
$$


With the help of the next lemma an argument by Pukánszky Puk71, p. 70] shows that the definition of $d(l)$ as above coincides with the one given, for example, in BBL16, by jump indices.

Lemma 3.32. Let $b: V \times V \rightarrow \mathbb{R}$ be a skew-symmetric bilinear form, $V^{\perp}$ its radical and $W \subset V$ a subspace. Then

$$
\operatorname{dim}(W)+\operatorname{dim}\left(W^{\perp}\right)=\operatorname{dim}(V)+\operatorname{dim}\left(W \cap V^{\perp}\right) .
$$

Lemma 3.33. The dimensions in $d(l)$ decrease by steps of zero or one. There is a jump, that is, $d_{i-1}(l)=d_{i}(l)+1$ if and only if

$$
X_{i} \notin \mathfrak{g}_{l}+\operatorname{span}\left\{X_{i+1}, \ldots, X_{n}\right\} .
$$

Proof. The orthogonal complement of $\mathfrak{g}_{l}+\operatorname{span}\left\{X_{i+1}, \ldots, X_{n}\right\}$ with respect to the bilinear form $b_{l}$ is $\mathfrak{g}_{p_{i}(l)}$. Hence, by Lemma 3.32 there is a change of dimension if and only if the dimension of the orthogonal complement decreases. This is the case if and only if $X_{i} \notin \mathfrak{g}_{l}+\operatorname{span}\left\{X_{i+1}, \ldots, X_{n}\right\}$.

Let $D$ denote the finite set of all dimension sequences that occur for $G$. Assemble all $l \in \mathfrak{g}^{*} \backslash\{0\}$ with the same sequence to

$$
\Omega_{d}=\left\{l \in \mathfrak{g}^{*} \backslash\{0\} \mid d(l)=d\right\}
$$

for $d \in D$. The sets $\Omega_{d}$ are $G$-invariant because the projections $p_{i}$ are equivariant. As $\mathfrak{g}_{l}=\mathfrak{g}_{\lambda \cdot l}$ for all $\lambda \in \mathbb{R}_{>0}$, Lemma 3.33 implies that they are also invariant under the dilation action. For $d=\left(d_{1}, \ldots, d_{n}\right) \in D$ set $d_{n+1}=0$ and define

$$
\begin{aligned}
S(d) & =\left\{i \in\{1, \ldots, n\} \mid d_{i}=d_{i+1}+1\right\}, \\
T(d) & =\left\{i \in\{1, \ldots, n\} \mid d_{i}=d_{i+1}\right\}, \\
\mathfrak{g}_{S(d)}^{*} & =\operatorname{span}\left\{X_{i}^{*} \mid i \in S(d)\right\} \\
\mathfrak{g}_{T(d)}^{*} & =\operatorname{span}\left\{X_{i}^{*} \mid i \in T(d)\right\} .
\end{aligned}
$$

The following theorem is due to Pukánszky Puk71 and is also proved in CG90.

TheOREM 3.34 ( $\mathbf{C G 9 0}, 3.1 .14])$. There is an ordering $d_{1} \geq d_{2} \geq \ldots \geq d_{m}$ of $D$ such that all $W_{i}=\bigcup_{d \geq d_{i}} \Omega_{d}$ for $i=1, \ldots, m$ are $G$ - and $\mathbb{R}_{>0}$-invariant and open. Each $G$-orbit in $\Omega_{d}$ meets $\mathfrak{g}_{T(d)}^{*}$ in exactly one point.

This allows to find a sequence as in 12 using Kirillov's map by setting $V_{i}=$ $G \backslash W_{i}$ for $i=1, \ldots, m$. It remains to check that the $V_{i} \backslash V_{i-1}=G \backslash \Omega_{d_{i}}$ are Hausdorff and that the corresponding $\mathbb{R}_{>0}$-action is free and proper.

Proposition 3.35. For $d \in D$ let $\Lambda_{d}:=\Omega_{d} \cap \mathfrak{g}_{T(d)}^{*}$. The map $\Lambda_{d} \rightarrow G \backslash \Omega_{d}$ induced by the inclusion is an $\mathbb{R}_{>0}$-equivariant homeomorphism. The corresponding $\mathbb{R}_{>0}$-action on $\Lambda_{d}$ is free and proper.

Proof. In CG90, 3.1.14] it is proved that there is a birational, nonsingular map $\psi_{d}: \Lambda_{d} \times \mathfrak{g}_{S(d)}^{*} \rightarrow \Omega_{d}$. Furthermore, $\pi_{1} \circ \psi_{d}^{-1}$ is $G$-invariant, where $\pi_{1}$ denotes the projection to $\Lambda_{d}$. Hence, it induces a continuous map $G \backslash \Omega_{d} \rightarrow \Lambda_{d}$. It is inverse to the map induced by the inclusion. Thus, the two spaces are homeomorphic. As $\Omega_{d}$ and $\mathfrak{g}_{T(d)}^{*}$ are invariant under the dilation action, so is $\Lambda_{d}$. Therefore, the inclusion is equivariant. Since $0 \in \mathfrak{g}^{*}$ is not contained in any $\Omega_{d}$, the $\Lambda_{d}$ are subsets of some $\mathbb{R}^{l} \backslash\{0\}$ equipped with the Euclidean subspace topology. Hence they are Hausdorff and the $\mathbb{R}_{>0}$-action, which is given for $\lambda>0$ by multiplying the coordinate entries by different powers of $\lambda$, is free and proper. 
Example 3.36. From the computations for the Heisenberg group in Example 3.31 we get as in [CG90 3.1.15], up to the reversed order,

$$
\begin{aligned}
\Omega_{(2,1,0)} & =\left\{\alpha X^{*}+\beta Y^{*}+\gamma Z^{*} \mid \alpha, \beta \in \mathbb{R} \text { and } \gamma \neq 0\right\}, \\
\Omega_{(0,0,0)} & =\left\{\alpha X^{*}+\beta Y^{*} \mid(\alpha, \beta) \neq(0,0)\right\}, \\
T(2,1,0) & =\{3\}, \\
T(0,0,0) & =\{1,2,3\}, \\
\Lambda_{(2,1,0)} & =\left\{\gamma Z^{*} \mid \gamma \neq 0\right\}, \\
\Lambda_{(0,0,0)} & =\left\{\alpha X^{*}+\beta Y^{*} \mid(a, b) \neq(0,0)\right\} .
\end{aligned}
$$

Therefore, the desired sequence is $\emptyset \subset G \backslash \Omega_{(2,1,0)} \subset \widehat{G} \backslash\left\{\pi_{\text {triv }}\right\}$. The dilation action on $\Lambda_{(2,1,0)} \cong \mathbb{R} \backslash\{0\}$ is multiplication with $\lambda^{-2}$ for $\lambda>0$, whereas on $\Lambda_{(0,0,0)} \cong \mathbb{R}^{2} \backslash\{0\}$ it is scalar multiplication with $\lambda^{-1}$.

\subsection{Plancherel theory}

For a locally compact group $G$, the operator-valued Fourier transform $f \mapsto \widehat{f}$ is defined for $f \in L^{1}(G)$. It is given by

$$
\widehat{f}(\pi)=\int_{G} f(x) \pi(x) \mathrm{d} x \in \mathbb{B}\left(\mathcal{H}_{\pi}\right)
$$

for an irreducible, unitary representation $\pi: G \rightarrow \mathcal{U}\left(\mathcal{H}_{\pi}\right)$. We recall some results from Plancherel theory for locally compact, separable groups $G$ of type I (see [Dix77, 18.8], [CG90, 4.3] and [FR16, Appendix B]).

In this case, the topological space $\widehat{G}$ can be equipped with a certain Borel measure $\mu$, which is called the Plancherel measure. For a simply connected nilpotent Lie group $G$, it is supported within the orbits with maximal dimension sequence, these are the orbits in $\Omega_{d_{1}} \subset \mathfrak{g}^{*}$ with notation as in Theorem 3.34 (see CG90 p. 154]).

By Dix77, 8.6.1] there is a subspace $\Gamma \subset \prod_{\pi \in \widehat{G}} \mathcal{H}_{\pi}$ that turns $\left(\left(\mathcal{H}_{\pi}\right)_{\pi \in \widehat{G}}, \Gamma\right)$ into a $\mu$-measurable field of Hilbert spaces over $\widehat{G}$ as defined in FR16, Def. B.1.3]. The elements of $\Gamma$ are called the measurable sections.

For a Hilbert space $\mathcal{H}$ denote by $\operatorname{HS}(\mathcal{H})$ the Hilbert space of Hilbert-Schmidt operators on $\mathcal{H}$. Identifying $\mathcal{H} \otimes \mathcal{H}^{*}$ with $\operatorname{HS}(\mathcal{H})$, one obtains for a simply connected nilpotent Lie group $G$ the $\mu$-measurable field $\left(\left(\operatorname{HS}\left(\mathcal{H}_{\pi}\right)\right)_{\pi \in \widehat{G}}, \Gamma \otimes \Gamma^{*}\right)$ over $\widehat{G}$ (see FR16, B.1.3]).

Define $L^{2}\left(\widehat{G}, \operatorname{HS}\left(\mathcal{H}_{\pi}\right)\right)$ to be the direct integral of the Hilbert spaces $\operatorname{HS}\left(\mathcal{H}_{\pi}\right)$. It consists of sections $x \in \Gamma \otimes \Gamma^{*}$ such that $(\pi \mapsto\|x(\pi)\|) \in L^{2}(\widehat{G}, \mu)$. It is a Hilbert space with respect to $\langle x, y\rangle:=\int_{\widehat{G}}\langle x(\pi), y(\pi)\rangle_{\mathrm{HS}} \mathrm{d} \mu(\pi)$.

The Plancherel Theorem [FR16, B.2.32] states that the Fourier transform defined above yields an isometric isomorphism

$$
\text { : } L^{2}(G) \rightarrow L^{2}\left(\widehat{G}, \operatorname{HS}\left(\mathcal{H}_{\pi}\right)\right)
$$

The (left) group von Neumann algebra $\mathrm{VN}_{L}(G)$ of $G$ consists of bounded, rightinvariant operators on $L^{2}(G)$. The Plancherel Theorem yields that the Fourier transform extends to a *-isomorphism

$$
\text { ^: } \operatorname{VN}_{L}(G) \rightarrow L^{\infty}\left(\widehat{G}, \mathbb{B}\left(\mathcal{H}_{\pi}\right)\right) \text {. }
$$

Here, $L^{\infty}\left(\widehat{G}, \mathbb{B}\left(\mathcal{H}_{\pi}\right)\right)$ consists of $a=(a(\pi))_{\pi \in \widehat{G}}$ with $a(\pi) \in \mathbb{B}\left(\mathcal{H}_{\pi}\right)$ such that

(i) $(a(\pi) x(\pi))_{\pi} \in \Gamma$ for all $(x(\pi))_{\pi} \in \Gamma$,

(ii) $(\pi \mapsto\|a(\pi)\|) \in L^{\infty}(\widehat{G}, \mu)$. 
It is a von Neumann-algebra with the pointwise operations and the norm given by

$$
\|a\|=\sup _{\pi \in \widehat{G}}\|a(\pi)\|_{\mathbb{B}\left(\mathcal{H}_{\pi}\right)} .
$$

The inverse Fourier transform maps a field $a \in L^{\infty}\left(\widehat{G}, \mathbb{B}\left(\mathcal{H}_{\pi}\right)\right)$ to the operator $T_{a} \in \mathrm{VN}_{L}(G)$ determined by

$$
\widehat{T_{a} \psi}(\pi)=a(\pi) \widehat{\psi}(\pi) \quad \text { for } \psi \in L^{2}(G), \pi \in \widehat{G} .
$$

This operator-valued Fourier transform is essential for the definition of the symbolic pseudodifferential calculus of Fischer-Ruzhansky described in Section 9.2 and will also be useful at some other places in the following. 


\section{CHAPTER 4}

\section{Filtered manifolds}

For a differential operator $P$ of order $m$ on an ordinary smooth manifold, its highest order part $\Sigma(P)$ is independent of the choice of coordinates. If $\left(x_{1}, \ldots, x_{n}\right)$ are local coordinates on $M$ one can write

$$
P=\sum_{|\alpha| \leq m} c_{\alpha} \partial^{\alpha}
$$

with smooth coefficients $c_{\alpha} \in \mathrm{C}^{\infty}(M)$. In these coordinates, the highest order part, or principal part, at $x \in M$ is

$$
\Sigma(P)_{x}=\sum_{|\alpha|=m} c_{\alpha}(x) \partial^{\alpha} .
$$

For a fixed $x \in M$, the "model operator" $\Sigma(P)_{x}$ can be understood as a constant coefficient operator on $\mathbb{R}^{n}$. Usually, it is more convenient to apply Fourier transform: $\widehat{\Sigma(P)_{x}}$ is acting by multiplication with the polynomial

$$
\sum_{|\alpha|=m} c_{\alpha}(x)(-i \xi)^{\alpha}
$$

Using Fourier transform, it becomes easy to say when $\Sigma(P)_{x}$ is invertible and to construct an inverse in this case. On a compact manifold $M, P$ is called elliptic if $\widehat{\Sigma(P)_{x}}(\xi) \neq 0$ for all $\xi \neq 0$ and $x \in M$.

Folland, Rothschild and Stein realized in the 1970s [FS74, RS76, Fol77 that certain non-elliptic, but still hypoelliptic, differential operators could be analysed using graded Lie groups. By attaching different integer weights to vector fields viewed as differential operators, one obtains a new notion of order. Then the model operators of the highest order part act no longer on $\mathbb{R}^{n}$ but on graded Lie groups instead. The Kohn-Laplacian $\square_{b}$ and operators of the type of Hörmander's sum of squares are examples studied by Folland, Rothschild and Stein.

Filtered manifolds, which are also called Carnot manifolds, offer a framework to study this behaviour. The tangent bundle of a filtered manifold admits a filtration by subbundles. This notion goes back to Tan70. It allows to attach different weights to vector fields in a well-defined way. In the last years, filtered manifolds were used in the realm of noncommutative geometry in vEY19, vEY17, CP19b, SH18, Moh18, Moh20.

In this chapter, we recall the definition of a filtered manifold and give some examples. For each point of a filtered manifold, the filtration yields a graded nilpotent Lie group, the so called osculating group. In the last section, we consider certain coordinates defined in $\mathbf{C P 1 9 a}$. These give a better understanding how the osculating groups approximate the manifold in a suitable sense.

Definition 4.1. A filtered manifold $(M, H)$ is a smooth manifold with a filtration of its tangent bundle $0=H^{0} \subseteq H^{1} \subseteq H^{2} \subseteq \ldots \subseteq H^{r}=T M$ consisting of smooth subbundles such that

$$
\left[\Gamma^{\infty}\left(H^{i}\right), \Gamma^{\infty}\left(H^{j}\right)\right] \subseteq \Gamma^{\infty}\left(H^{i+j}\right) \text { for all } i, j .
$$


Here, we set $H^{i}=T M$ for all $i \geq r$. A manifold is filtered of step $r$, if $H^{r}=T M$ and $H^{r-1} \neq T M$.

We consider some examples. For a broader overview on different types of filtered manifolds appearing in various areas of mathematics see CP17, Section 2.3].

Example 4.2. A filtered manifold of step $r=1$ is just the data of an ordinary smooth manifold.

Example 4.3. By the Frobenius Theorem a foliated manifold is a manifold with a subbundle $0 \subseteq H \subseteq T M$ such that $\left[\Gamma^{\infty}(H), \Gamma^{\infty}(H)\right] \subset \Gamma^{\infty}(H)$. In particular, this defines a filtered manifold of step 2 .

Example 4.4. A manifold with a step 2 filtration $0 \subsetneq H \subsetneq T M$ such that $H$ has codimension 1 is called a Heisenberg manifold. A contact manifold is a $(2 k+1)$ dimensional Heisenberg manifold such that for all local 1-forms $\theta$ with $\operatorname{ker}(\theta)=H$ the $(2 k+1)$-form $\theta(\mathrm{d} \theta)^{k}$ is nowhere zero on its domain.

Example 4.5. Each graded Lie group $G$ of step $r$ can be understood as a filtered manifold of step $r$. Suppose $T_{0}(G)=\mathfrak{g}=\oplus_{i=1}^{r} \mathfrak{g}_{i}$ and let $n_{k}=\sum_{i=1}^{k} \operatorname{dim} \mathfrak{g}_{i}$ for $k \geq 1$ and $n_{0}=0$. Choose a basis $\left\{X_{1}, \ldots, X_{n}\right\}$ of $\mathfrak{g}$ by subsequently picking a basis $\left\{X_{n_{i-1}+1}, \ldots, X_{n_{i}}\right\}$ of $\mathfrak{g}_{i}$. Extend these to left-invariant vector fields $\left\{X_{1}, \ldots, X_{n}\right\}$ on $G$ and define $H^{i}$ to be the subbundle spanned by $\left\{X_{1}, \ldots, X_{n_{i}}\right\}$. This defines a filtration of the tangent bundle of $G$.

\subsection{The osculating groupoid}

The filtration of the tangent bundle allows to define the graded vector bundle

$$
\bigoplus_{i=1}^{\infty} H^{i} / H^{i-1}
$$

over $M$. Note that $H^{i} / H^{i-1}=0$ for $i>r$, where $r$ is the step of the filtration. The graded bundle can be equipped with the structure of a Lie algebroid over $M$, which we will denote by $\mathfrak{t}_{H} M$. For the general theory of Lie algebroids and Lie groupoids see, for example, Mac05]. The bracket

$$
[\cdot, \cdot]: \Gamma^{\infty}\left(\mathfrak{t}_{H} M\right) \times \Gamma^{\infty}\left(\mathfrak{t}_{H} M\right) \rightarrow \Gamma^{\infty}\left(\mathfrak{t}_{H} M\right)
$$

is induced by the Lie bracket of vector fields on $T M$. Let $X \in \Gamma^{\infty}\left(H^{i}\right)$ and $Y \in \Gamma^{\infty}\left(H^{j}\right)$ be representatives of $\langle X\rangle \in \Gamma^{\infty}\left(H^{i} / H^{i-1}\right)$ and $\langle Y\rangle \in \Gamma^{\infty}\left(H^{j} / H^{j-1}\right)$ and set

$$
[\langle X\rangle,\langle Y\rangle]=\langle[X, Y]\rangle \text {. }
$$

The condition (14 ensures that this is well-defined. The anchor $\mathfrak{t}_{H} M \rightarrow T M$ is given by the zero map. Therefore, the bracket restricts to each fibre $\left(\mathfrak{t}_{H} M\right)_{x}$ for $x \in M$ and turns $\left(\mathfrak{t}_{H} M\right)_{x}$ into a graded Lie algebra.

The Lie algebroid $\mathfrak{t}_{H} M$ integrates to a Lie groupoid $T_{H} M$ over $M$ (see vEY17, Sec. 3, Sec. 8]). As a manifold, it is the graded vector bundle $\bigoplus_{i=1}^{\infty} H^{i} / H^{i-1}$. Its source and range map are the base projection.

Definition 4.6. For $x \in M$ denote by $G(x)$ the graded Lie group of $\left(\mathfrak{t}_{H} M\right)_{x}$ and call it the osculating group at $x \in M$.

The group multiplication of $G(x)$ is uniquely determined in terms of the Lie bracket by the Baker-Campbell-Hausdorff formula (5). The groupoid multiplication in $T_{H} M$ is given by the group product in the fibres. As the brackets vary smoothly along $M$, this defines a Lie groupoid multiplication. The Lie groupoid $T_{H} M$ is called the osculating groupoid or the bundle of osculating groups in $\mathbf{v E Y 1 7}$. In $\mathbf{C P 1 9 b}$ it is called the tangent group bundle. However, $T_{H} M$ is in general not 
a group bundle in the sense of principal bundles as the group structure might vary from point to point.

Example 4.7. Let $M=\mathbb{R}^{3}$ and define three vector fields

$$
X=\partial_{x}+y^{2} \partial_{z}, \quad Y=\partial_{y} \text { and } \quad Z=\partial_{z} .
$$

Let $H \subset T \mathbb{R}^{3}$ be the subbundle spanned by $X, Y$. One computes $[X, Y]=-2 y Z$. Therefore, the osculating groups are Abelian whenever $y=0$, while they are isomorphic to a Heisenberg group for $y \neq 0$.

Example 4.8. For step $r=1$ and $x \in M$, the osculating group $G(x)$ is the tangent space $T_{x} M$ with group operation being the addition of tangent vectors. Hence, all osculating groups are isomorphic to the Abelian group $\mathbb{R}^{n}$.

Example 4.9. The osculating groups of a foliated manifold are also Abelian as $\left[\Gamma^{\infty}(H), \Gamma^{\infty}(H)\right] \subset \Gamma^{\infty}(H)$ holds.

Example 4.10. For a Heisenberg manifold $M$, despite the name, the osculating groups are not necessarily Heisenberg groups. In fact, each osculating group $G(x)$ is isomorphic to a product $\mathbb{R}^{l(x)} \times \mathbb{H}_{k(x)}$ with $l(x)+2 k(x)+1=\operatorname{dim}(M)$ BG88, 1.15]. Note that $l(x)$ and $k(x)$ might vary along $M$. For a contact manifold of dimension $2 k+1$ all osculating groups are isomorphic to the Heisenberg group $\mathbb{H}_{k}$.

Example 4.11. For a graded Lie group $G$, understood as a filtered manifold as in Example 4.5 all osculating groups are isomorphic to $G$.

Definition 4.12. The dilation action of $\mathbb{R}_{>0}$ on the graded Lie algebras $\left(\mathfrak{t}_{H} M\right)_{x}$ extends to an action on $\mathfrak{t}_{H} M$ given by $D_{\lambda}(\langle X\rangle):=\lambda^{i}\langle X\rangle$ for $X \in H_{x}^{i} / H_{x}^{i-1}$. It integrates to an action on $T_{H} M$, which we denote by $\delta_{\lambda}(\xi)=\lambda \cdot \xi$ for $\xi \in G(x)$.

From now on, we will always assume that the bundles $H^{i}$ of the filtration of a filtered manifold $(M, H)$ have constant rank, which is automatic if $M$ is connected.

Definition 4.13. The homogeneous dimension of a filtered manifold $(M, H)$ is

$$
d_{H}:=\sum_{i=1}^{n} i\left(\operatorname{rank}\left(H^{i}\right)-\operatorname{rank}\left(H^{i-1}\right)\right) .
$$

The weight sequence for $(M, H)$ is

$$
\left(q_{1}, \ldots, q_{n}\right)=(1, \ldots, 1,2, \ldots, 2, \ldots, r, \ldots, r)
$$

where each $1 \leq i \leq r$ occurs $\left(\operatorname{rank}\left(H^{i}\right)-\operatorname{rank}\left(H^{i-1}\right)\right)$-times.

Remark 4.14. The homogeneous dimension of the filtered manifold is the homogeneous dimension of all osculating groups $G(x)$ for $x \in M$ as in Definition 3.9 .

Assigning to a filtered manifold $(M, H)$ its osculating groupoid $T_{H} M$ is functorial, when considering the following morphisms.

Definition 4.15. A filtered manifold map or Carnot map $f:\left(M_{1}, H_{1}\right) \rightarrow\left(M_{2}, H_{2}\right)$ is a smooth map between filtered manifolds $\left(M_{1}, H_{1}\right)$ and $\left(M_{2}, H_{2}\right)$ such that

$$
\mathrm{d} f\left(H_{1}^{i}\right) \subset H_{2}^{i} \text { for all } i \in \mathbb{N} \text {. }
$$

A Carnot diffeomorphism is a diffeomorphism $f: M_{1} \rightarrow M_{2}$ such that $f$ and $f^{-1}$ are filtered manifold maps.

Let $f:\left(M_{1}, H_{1}\right) \rightarrow\left(M_{2}, H_{2}\right)$ be a Carnot map. Condition 15 ensures that there is a well-defined induced vector bundle morphism $\mathfrak{t} f: \mathfrak{t}_{H_{1}} M_{1} \rightarrow \mathfrak{t}_{H_{2}} M_{2}$, which satisfies

$$
[\mathfrak{t} f(X), \mathfrak{t} f(Y)]=\mathfrak{t} f([X, Y]) \quad \text { for } X, Y \in \Gamma^{\infty}\left(\mathfrak{t}_{H_{1}} M_{1}\right)
$$


Consequently, using the exponential maps, one obtains a Lie groupoid homomorphism $T f: T_{H_{1}} M_{1} \rightarrow T_{H_{2}} M_{2}$ which restricts in each fibre to a homomorphism of graded Lie groups $T_{x} f: G(x) \rightarrow G(f(x))$. The map $T f$ is equivariant for the dilation actions, that is,

$$
T_{x} f(\lambda \cdot \xi)=\lambda \cdot T_{x} f(\xi) \quad \text { for } x \in M, \lambda>0 \text { and } \xi \in G(x) .
$$

We will consistently use the notation $\mathrm{d} f: T M_{1} \rightarrow T N_{1}$ for the usual differential, whereas $T f: T_{H_{1}} M_{1} \rightarrow T_{H_{2}} M_{2}$ is the homomorphism between the osculating groupoids, which is called the Carnot differential in $\mathbf{C P 1 9 b .}$.

Proposition $4.16(\mathbf{C P 1 9 b}, 5.5])$. The assignment $(M, H) \mapsto T_{H} M$ and $f \mapsto T f$ defines a functor from the category of filtered manifolds with filtered manifold maps to the category of Lie groupoids with Lie groupoid homomorphisms.

\subsection{Anisotropic analysis}

In this section, let $\mathbb{R}^{n}$ be equipped with dilations $\delta: \mathbb{R}_{>0} \curvearrowright \mathbb{R}^{n}$ of integer weights $1=q_{1} \leq \ldots \leq q_{n}$, that is,

$$
\delta_{\lambda}\left(v_{1}, \ldots, v_{n}\right)=\lambda \cdot\left(v_{1}, \ldots, v_{n}\right):=\left(\lambda^{q_{1}} v_{1}, \ldots, \lambda^{q_{n}} v_{n}\right) \text { for } \lambda>0, v \in \mathbb{R}^{n} .
$$

We will recall some facts and notions on anisotropic analysis for $\mathbb{R}^{n}$ equipped with these dilations. They were introduced in $[\mathbf{C P 1 7}, \mathbf{C P 1 9 a}$ to define certain coordinates on filtered manifolds, which we will describe in Section 4.3

Fix a homogeneous quasi-norm $\|\cdot\|$ as in Definition 3.11 for the dilations above. Note that one only needs dilations and does not need to specify an underlying group structure to define them. This is a key observation as the group product in the osculating groups can change along the manifold, whereas the dilations stay the same. For a multiindex $\alpha \in \mathbb{N}_{0}^{n}$ we denote by $[\alpha]$ the homogeneous degree as in Definition 3.15

Definition 4.17 ([CP17, 4.4]). Let $U \subset \mathbb{R}^{n}$ be an open neighbourhood of $0 \in \mathbb{R}^{n}$ and $m \in \mathbb{N}_{0}$. A function $f \in \mathrm{C}^{\infty}(U)$ has weight $\geq m$ at 0 if $\partial^{\alpha} f(0)=0$ for all $\alpha \in \mathbb{N}_{0}^{n}$ with $[\alpha]<m$.

By [CP17, 4.12] $f \in \mathrm{C}^{\infty}(U)$ has weight $\geq m$ at 0 if and only if $f$ has the asymptotic behaviour $f=O\left(\|v\|^{m}\right)$ near $v=0$. To deal with vector-valued maps, the following definition is useful.

Definition 4.18 ( CP17, 4.18]). Let $U \subset \mathbb{R}^{n}$ be an open neighbourhood of $0 \in \mathbb{R}^{n}$ and let $\mathbb{R}^{k}$ be equipped with dilations with integer weights $1=q_{1}^{\prime} \leq \ldots \leq q_{k}^{\prime}$. A function $f=\left(f_{1}, \ldots, f_{k}\right) \in \mathrm{C}^{\infty}\left(U, \mathbb{R}^{k}\right)$ belongs to $O_{q}\left(\|v\|^{q^{\prime}+m}\right)$ for $m \in \mathbb{N}_{0}$ if

$$
f_{i}=O\left(\|v\|^{q_{i}^{\prime}+m}\right) \quad \text { near } v=0 \text { for all } i=1, \ldots, k .
$$

The following lemma will be used later for computations on the tangent groupoid of a filtered manifold.

Lemma $4.19(\mathbf{C P 1 9 b}, 3.9])$. Let $W$ is an open subset of $\mathbb{R}^{p} \times \mathbb{R}^{n}$ such that $W \cap\left(\mathbb{R}^{p} \times\{0\}\right)=U \times\{0\}$ for a non-empty $U \subseteq \mathbb{R}^{p}$. Let $\Theta \in \mathrm{C}^{\infty}\left(W, \mathbb{R}^{n}\right)$ satisfy $\Theta(x, v)=O_{q}\left(\|v\|^{q+m}\right)$ near $v=0$ for all $x \in U$. Then there are smooth maps $R_{i, \alpha} \in \mathrm{C}^{\infty}(W)$ such that for $i=1, \ldots, n$

$$
\left(t^{-1} \cdot \Theta(x, t \cdot v)\right)_{i}=\sum_{|\alpha| \leq q_{i}+m \leq[\alpha]} t^{[\alpha]-q_{i}} v^{\alpha} R_{i, \alpha}(x, t \cdot v) .
$$


Proof. For a function $f \in \mathrm{C}^{\infty}(W, \mathbb{R})$ and $N \in \mathbb{N}_{0}$ the anisotropic Taylor formula [CP17, 4.7] yields functions $R_{N, \alpha} \in \mathrm{C}^{\infty}(W, \mathbb{R})$ such that

$$
f(x, v)=\sum_{[\alpha]<N} \frac{1}{\alpha !} v^{\alpha} \partial_{v}^{\alpha} f(x, 0)+\sum_{|\alpha| \leq N \leq[\alpha]} v^{\alpha} R_{N, \alpha}(x, v)
$$

for all $(x, v) \in W$. If $f(x, v)=O\left(\|v\|^{N}\right)$ near $v=0$ for all $x \in U$, it follows that

$$
f(x, t \cdot v)=\sum_{|\alpha| \leq N \leq[\alpha]} t^{[\alpha]} v^{\alpha} R_{N, \alpha}(x, t \cdot v) .
$$

The claim follows by considering for $i=1, \ldots, n$ the components $\Theta_{i} \in \mathrm{C}^{\infty}(W, \mathbb{R})$ and $N=q_{i}+m$.

As in (8), the dilations act on differential operators on $\mathbb{R}^{n}$ and, in particular, on vector fields. For a differential operator $P$ on $\mathbb{R}^{n}$ set

$$
\delta_{\lambda}^{*}(P) f(v)=P\left(f \circ \delta_{\lambda}\right)\left(\lambda^{-1} \cdot v\right) \quad \text { for } \lambda>0, f \in \mathrm{C}^{\infty}\left(\mathbb{R}^{n}\right) \text { and } v \in \mathbb{R}^{n} .
$$

Definition 4.20. A differential operator $P$ on $\mathbb{R}^{n}$ is $q$-homogeneous with respect to the dilations $\delta: \mathbb{R}_{>0} \curvearrowright \mathbb{R}^{n}$ if $\delta_{\lambda}^{*}(P)=\lambda^{q} P$.

\subsection{Coordinates}

As already remarked, for a filtered manifold the osculating groups may vary from point to point. Therefore, it is not always possible to identify $M$ locally with open subsets of a fixed graded Lie group. This can only be done in particular cases, for example for contact manifolds using Darboux coordinates.

However, there are special kinds of coordinates on filtered manifolds that give a relation with the group structure of the osculating groups. Using $H$-charts the bundle $T_{H} M$ can be locally trivialized in a way that is compatible with the graded structure.

Definition 4.21. An $H$-frame $X=\left(X_{1}, \ldots, X_{n}\right)$ over an open subset $V \subset M$ consists of vector fields $X_{i}: V \rightarrow T M$, such that $\left(X_{1}, \ldots, X_{\text {rank } H^{i}}\right)$ defines a frame for $H^{i}$ for all $i=1, \ldots, r$.

An $H$-chart is a local chart $\kappa: V \rightarrow U$ between open subsets $V \subset M$ and $U \subset \mathbb{R}^{n}$ together with an $H$-frame $X$ over $V$.

For an $H$-chart $\kappa: V \rightarrow U$ with $H$-frame $X$, the frame $\left\{\kappa_{*}\left(X_{1}\right), \ldots, \kappa_{*}\left(X_{n}\right)\right\}$ induces a filtration over $U$ we denote by $\kappa_{*} H$. Therefore, $\kappa$ can be understood as a Carnot diffeomorphism. The Carnot differential $T \kappa$ gives an isomorphism of bundles of graded groups

$$
T \kappa:\left.T_{H} M\right|_{V} \rightarrow T_{\kappa_{*} H} U .
$$

We define a fibrewise homogeneous quasi-norm on $T_{\kappa_{*} H} U$ as in (6) by

$$
\|(x, v)\|:=\sum_{j=1}^{n}\left|v_{j}\right|^{1 / q_{j}} \quad \text { for } x \in U, v \in \mathbb{R}^{n} .
$$

For a fixed point $p \in M$, we consider now the privileged and Carnot coordinates at $p$. See also [SH18 6.21, 6.22, 6.23] for a different description of these.

Definition 4.22 ([CP17, 3.8, 3.10]). Let $\kappa: V \rightarrow U$ be an $H$-chart around $p \in M$ with coordinate functions $x_{j}: V \rightarrow \mathbb{R}$ and $H$-frame $X$. The $H$-chart is linearly adapted to $p$ if $\kappa(p)=0$ and the $H$-frame coincides with the coordinate vector fields at $p$, that is,

$$
X_{j}(p)=\frac{\partial}{\partial x_{j}}(p) \quad \text { for } j=1, \ldots, n \text {. }
$$


For an $H$-chart $\kappa: V \rightarrow U$, equip $\mathbb{R}^{n}$ with the dilations induced by $T \kappa$. Then one can speak of vector fields on $U \subset \mathbb{R}^{n}$ that are homogeneous with respect to the dilations in the sense of Definition 4.20

Definition $4.23([\mathbf{C P 1 7}, 5.6])$. An $H$-chart $\kappa: V \rightarrow U$ with $H$-frame $X$ which is linearly adapted to $p \in V$ defines privileged coordinates at $p$ if for all $j=1, \ldots, n$ there is a $q_{j}$-homogeneous vector field $X_{j}^{[p]}$ on $U$ such that

$$
t^{q_{j}} \delta_{t^{-1}}^{*}\left(\kappa_{*}\left(X_{j}\right)\right)=X_{j}^{[p]}+O(t) \quad \text { in } \Gamma^{\infty}(U) \text { as } t \rightarrow 0 .
$$

Here, $\Gamma^{\infty}(U)$ is equipped with the smooth structure under the identification with $\mathrm{C}^{\infty}\left(U, \mathbb{R}^{n}\right)$ using the coordinate vector fields.

Using an $H$-chart $\kappa: V \rightarrow U$ with $H$-frame $X, T \kappa$ identifies $\left.T_{H} M\right|_{V}$ with a bundle of graded Lie groups over $U$. The frame $\left(\kappa_{*}\left(X_{1}\right), \ldots, \kappa_{*}\left(X_{n}\right)\right)$ yields a basis of the graded Lie algebra over $\kappa(p) \in U$. Let $X_{j}^{p}$ for $j=1, \ldots, n$ denote the left-invariant vector fields associated to this basis as in Definition 3.18 using the multiplication over $\kappa(p)$. Then each $X_{j}^{p}$ is a $q_{j}$-homogeneous vector field on $U$.

Definition 4.24 ( $\mathbf{C P 1 9 a}, 7.7])$. An $H$-chart $\kappa: V \rightarrow U$ with $H$-frame $X$ which is linearly adapted to $p \in V$ defines Carnot coordinates at $p$ if for all $j=1, \ldots, n$

$$
t^{q_{j}} \delta_{t^{-1}}^{*}\left(\kappa_{*}\left(X_{j}\right)\right)=X_{j}^{p}+O(t) \quad \text { in } \Gamma^{\infty}(U) \text { as } t \rightarrow 0 .
$$

Therefore, Carnot coordinates are the privileged coordinates at $p$ for which the "blow up" in (17) yields the left-invariant differential operators obtained by the $H$-frame $X$ on the osculating group at $p$. This explains why these coordinates will be important for the construction of the tangent groupoid.

Choi and Ponge give in CP19a an explicit construction how to turn any local coordinates into Carnot coordinates. The result can be summarized as follows.

Proposition 4.25 ([CP19b 4.17]). Let $\kappa: V \rightarrow U$ be an $H$-chart around $p \in M$ with $H$-frame $X$. Then there is a unique change of coordinates $\varepsilon_{\kappa(p)}^{\kappa}: \mathbb{R}^{n} \rightarrow \mathbb{R}^{n}$ such that $\varepsilon_{\kappa(p)}^{\kappa} \circ \kappa$ are Carnot coordinates at $p$ with respect to the $H$-frame $X$.

Moreover, the map $\varepsilon_{p}^{\kappa}: \mathbb{R}^{n} \rightarrow \mathbb{R}^{n}$ for $p \in U$ can be described as follows. Write the vector fields $\kappa_{*}\left(X_{j}\right)$ for $j=1, \ldots, n$ in terms of the coordinate vector fields as

$$
\kappa_{*}\left(X_{j}\right)=\sum_{k=1}^{n} b_{j k} \frac{\partial}{\partial x_{k}} \quad \text { with } b_{j k} \in \mathrm{C}^{\infty}(U) .
$$

Let $B_{X}(x):=\left(b_{j k}(x)\right)_{j, k=1}^{n}$ for $x \in U$ and define the affine linear map

$$
A_{p}(x):=\left(B_{X}(p)^{t}\right)^{-1}(x-p) \text {. }
$$

Then one can write $\varepsilon_{p}^{\kappa}$ by $\left.\mathbf{C P 1 9 a} 10.1\right]$

$$
\varepsilon_{p}^{\kappa}=\widehat{\varepsilon}_{p}^{\kappa} \circ A_{p}
$$

where $\widehat{\varepsilon}_{p}^{\kappa}$ is a polynomial map given by

$$
\widehat{\varepsilon}_{p}^{\kappa}(x)_{j}=x_{j}+\sum_{\substack{[\alpha]<q_{j},|\alpha| \geq 2}} c_{j \alpha}(p) x^{\alpha} \quad \text { for } j=1, \ldots, n .
$$

The $c_{j \alpha}$ are universal polynomials in the entries of $\left(B_{X}(p)^{t}\right)^{-1}$ and $\partial_{x}^{\beta} B_{X}(p)$ for $[\beta]<[\alpha]-1$. Varying $p$ inside $U$ we obtain the $\varepsilon$-Carnot map $\varepsilon^{\kappa}: U \times \mathbb{R}^{n} \rightarrow \mathbb{R}^{n}$ with $(p, x) \mapsto \varepsilon_{p}^{\kappa}(x)$.

Corollary $4.26([\mathbf{C P 1 9 a}, 10.4])$. The maps $U \times \mathbb{R}^{n} \rightarrow \mathbb{R}^{n}$ given by $(p, x) \mapsto \varepsilon_{p}^{\kappa}(x)$ and $(p, x) \mapsto\left(\varepsilon_{p}^{\kappa}\right)^{-1}(x)$ are smooth. 
The $\varepsilon$-Carnot maps approximate the group multiplication of the osculating groups in the following sense.

Proposition 4.27 ( around $p \in V$ with respect to the $H$-frame $X$. Then for $(x, y)$ near $(0,0)$

$$
\begin{aligned}
\varepsilon_{x}^{\kappa}(y) & =(-x) \cdot{ }_{p} y+O_{q}\left(\|(x, y)\|^{q+1}\right), \\
\left(\varepsilon_{x}^{\kappa}\right)^{-1}(y) & =x \cdot{ }_{p} y+O_{q}\left(\|(x, y)\|^{q+1}\right) .
\end{aligned}
$$

Here, $\cdot p$ denotes the group product in the osculating group at $\kappa(p)$. 


\section{CHAPTER 5}

\section{The tangent groupoid of a filtered manifold}

The tangent groupoid of a filtered manifold and its smooth structure were constructed in vEY19, CP19b, SH18, Moh18 using different approaches. It generalizes Connes' tangent groupoid [Con94], which plays an important role in Connes' proof of the Atiyah-Singer Index Theorem. It is a smooth field of groupoids over $[0, \infty)$ where the fibres are the osculating groupoid $T_{H} M$ at $t=0$ and the pair groupoid of $M$ for $t>0$. In case of a step $r=1$ filtration, one recovers Connes' tangent groupoid.

\subsection{The Lie groupoid structure}

Before defining the tangent groupoid of a filtered manifold, recall that the bundle of osculating groups $T_{H} M$ can be understood as a Lie groupoid over $M$.

Example 5.1. The bundle of osculating groups is a Lie groupoid with the following structure maps. The range and source map are given by the bundle projection $T_{H} M \rightarrow M$. The unit map is the embedding $M \hookrightarrow T_{H} M$ as the zero section. The inverse and multiplication are given by the group operations in the fibres.

Another important groupoid attached to $M$ is its pair groupoid.

Example 5.2. For a set $M$, the pair groupoid of $M$ is the groupoid with arrow space $M \times M$ and unit space $M$. The range and source $r, s: M \times M \rightarrow M$ and unit $u: M \rightarrow M \times M$ are given by

$$
r(x, y)=x, \quad s(x, y)=y, \quad u(x)=(x, x) .
$$

The inverse and multiplication are defined by

$$
(x, y)^{-1}=(y, x) \quad \text { and } \quad(x, y) \cdot(y, z)=(x, z) .
$$

If $M$ is a smooth manifold, the pair groupoid of $M$ is a Lie groupoid.

The two groupoids $T_{H} M$ and $M \times M$ can be glued together in a smooth way, yielding the tangent groupoid of $M$. We first discuss its groupoid structure.

Definition 5.3. The tangent groupoid $\mathbb{T}_{H} M$ of a filtered manifold $(M, H)$ consists of the arrow space

$$
\mathbb{T}_{H} M=\left(T_{H} M \times\{0\}\right) \cup(M \times M \times(0, \infty))
$$

and the unit space $M \times[0, \infty)$. The range and source maps $r, s: \mathbb{T}_{H} M \rightarrow M \times[0, \infty)$ are given by

$$
\begin{aligned}
& r(x, \xi, 0)=(x, 0), \quad s(x, \xi, 0)=(x, 0) \quad \text { for } \xi \in G(x), \\
& r(x, y, t)=(x, t), \quad s(x, y, t)=(y, t) \quad \text { for } x, y \in M \text { and } t>0 .
\end{aligned}
$$

The unit $u: M \times[0, \infty) \rightarrow \mathbb{T}_{H} M$ and the inverse $i: \mathbb{T}_{H} M \rightarrow \mathbb{T}_{H} M$ are defined by

$$
\begin{array}{clrl}
u(x, 0) & =(x, 0,0) \quad \text { for } x \in M, & u(x, t)=(x, x, t) & \text { for } x \in M \text { and } t>0, \\
i(x, \xi, 0)=\left(x, \xi^{-1}, 0\right) & \text { for } \xi \in G(x), & i(x, y, t)=(y, x, t) & \text { for } x, y \in M \text { and } t>0 .
\end{array}
$$


The multiplication $m: \mathbb{T}_{H} M^{(2)} \rightarrow \mathbb{T}_{H} M$ is given by

$$
\begin{aligned}
(x, \xi, 0)(x, \eta, 0) & =(x, \xi \cdot \eta, 0) & & \text { for } \xi, \eta \in G(x), \\
(x, y, t)(y, z, t) & =(x, z, t) & & \text { for } x, y, z \in M \text { and } t>0 .
\end{aligned}
$$

At $t=0$, the multiplication and inversion in the osculating groups are used.

The range fibres are given by

$$
\mathbb{T}_{H} M^{(x, t)}= \begin{cases}\{(x, \xi, 0) \mid \xi \in G(x)\} & \text { for } x \in M \text { and } t=0, \\ \{(x, y, t) \mid y \in M\} & \text { for } x \in M \text { and } t>0 .\end{cases}
$$

Example 5.4. Let $M=G$ be a graded Lie group with the filtration $H$ as in Example 4.5 The tangent groupoid $\mathbb{T}_{H} G$ is isomorphic to the transformation groupoid

$$
\mathcal{G}=(G \times[0, \infty)) \rtimes G
$$

of the action $(G \times[0, \infty)) \curvearrowleft G$ given by $(x, t) \cdot v=\left(x \delta_{t}(v), t\right)$. Here, we set $\delta_{0}(v)=$ $\lim _{t \rightarrow 0} \delta_{t}(v)=0$ for all $v \in G$.

The unit map $u: \mathcal{G}^{0}:=G \times[0, \infty) \rightarrow \mathcal{G}$, the range and source maps $r, s: \mathcal{G} \rightarrow \mathcal{G}^{0}$, the inverse and the multiplication are given by

$$
\begin{gathered}
u(x, t)=(x, t, 0), \quad r(x, t, v)=(x, t), \quad s(x, t, v)=\left(x \delta_{t}(v), t\right), \\
(x, t, v)^{-1}=\left(x \delta_{t}(v), t, v^{-1}\right), \quad(x, t, v) \cdot\left(x \delta_{t}(v), t, w\right)=(x, t, v w),
\end{gathered}
$$

for $x, v, w \in G$ and $t \in[0, \infty)$.

The isomorphism $\phi: \mathbb{T}_{H} G \rightarrow \mathcal{G}$ is given by $\phi(x, y, t)=\left(x, t, \delta_{t^{-1}}\left(x^{-1} y\right)\right)$ for $t>0$ and $\phi(x, \xi, 0)=(x, 0, \xi)$ when identifying $G(x)$ with $G$. The inverse is given by $(x, t, v) \mapsto\left(x, x \delta_{t}(v), t\right)$ for $t>0$ and $(x, 0, v) \mapsto(x, v, 0)$.

A crucial feature of the tangent groupoid of a filtered manifold is that it defines a Lie groupoid:

THEOREM 5.5 ( $\mathbf{v E Y 1 9 , \mathbf { C P 1 9 b }})$. The tangent groupoid $\mathbb{T}_{H} M$ of a filtered manifold $(M, H)$ admits a smooth structure such that it becomes a Lie groupoid.

We recall the construction of coordinate charts in $\mathbf{C P 1 9 b}$. For an $H$-chart $\kappa: M \supseteq V \rightarrow U \subseteq \mathbb{R}^{n}$, Choi and Ponge construct in [CP19b. (9.5)] a chart $\phi_{\kappa}: \mathbb{T}_{H} M \supseteq \mathbb{V} \rightarrow \mathbb{U}$, where

$$
\begin{aligned}
& \mathbb{V}=\left(\left.T_{H} M\right|_{V} \times\{0\}\right) \cup(V \times V \times(0, \infty)), \\
& \mathbb{U}=\left\{(x, v, t) \in U \times \mathbb{R}^{n} \times[0, \infty) \mid\left(\varepsilon_{x}^{\kappa}\right)^{-1}(t \cdot v) \in U\right\} .
\end{aligned}
$$

Here, $\varepsilon^{\kappa}$ is the $\varepsilon$-Carnot map $U \times \mathbb{R}^{n} \rightarrow \mathbb{R}^{n}$ associated with the $H$-chart $\kappa$. The map $\phi_{\kappa}$ is given by

$$
\begin{array}{ll}
\phi_{\kappa}(x, \xi, 0)=\left(\kappa(x), T_{x} \kappa(\xi), 0\right) & \text { for } \xi \in G(x), \\
\phi_{\kappa}(x, y, t)=\left(\kappa(x), t^{-1} \cdot \varepsilon_{\kappa(x)}^{\kappa}(\kappa(y)), t\right) & \text { for } x, y \in V \text { and } t>0 .
\end{array}
$$

Its inverse is

$$
\phi_{\kappa}^{-1}(x, v, t)= \begin{cases}\left(\kappa^{-1}(x),\left(T_{x} \kappa\right)^{-1}(v), 0\right) & \text { for }(x, v) \in U \times \mathbb{R}^{n} \text { and } t=0, \\ \left(\kappa^{-1}(x),\left(\varepsilon_{x}^{\kappa} \circ \kappa\right)^{-1}(t \cdot v), t\right) & \text { for }(x, v, t) \in \mathbb{U} \text { and } t>0 .\end{cases}
$$

The smooth structure of $\mathbb{T}_{H} M$ is uniquely determined by the charts $\phi_{\kappa}$ for all $H$-charts $\kappa$ of $M$ and by requiring that the inclusion $M \times M \times(0, \infty) \hookrightarrow \mathbb{T}_{H} M$ is a smooth embedding (see $\mathbf{C P 1 9 b}, 9.7]$ ). To shorten notation, we will sometimes denote $V_{\infty}=M$ and $\mathbb{V}_{\infty}=M \times M \times(0, \infty)$ in the following.

For each $H$-chart $\kappa: V \rightarrow U$, the open subset $\mathbb{V}$ is a subgroupoid of $\mathbb{T}_{H} M$. Hence, the structure maps from Definition 5.3 can be transported to $\mathbb{U}$ and one 
can check that they are smooth. Moreover, the unit map is a smooth embedding, the source and range maps are submersions and the inversion is a diffeomorphism. Hence, $\mathbb{T}_{H} M$ is a Lie groupoid.

Example 5.6. Let $G$ be a graded Lie group $G$ and let $\kappa: G \rightarrow \mathbb{R}^{n}$ be the global coordinate chart obtained from the exponential map. Then the $\varepsilon$-Carnot map is $\varepsilon_{x}^{\kappa}(y)=x^{-1} \cdot y$, see $\mathbf{C P 1 9 a}$ 9.12]. Therefore, $\phi_{\kappa}$ is the isomorphism from Example 5.4

\subsection{Smooth field structure}

Often, it will be useful to understand $\mathbb{T}_{H} M$ as a smooth field of groupoids over the space $[0, \infty)$ in the sense of $[$ LR01, 5.2].

Lemma 5.7. The tangent groupoid $\mathbb{T}_{H} M$ of a filtered manifold $(M, H)$ is a smooth field of groupoids over $[0, \infty)$ with fibres isomorphic to the pair groupoid of $M$ for $t>0$ and osculating groupoid $T_{H} M$ for $t=0$. All these subgroupoids are amenable.

Proof. The projection $\theta: \mathbb{T}_{H} M \rightarrow[0, \infty)$ is a smooth submersion. It satisfies $\theta=\operatorname{pr}_{2}$ or $=\operatorname{pr}_{2} \circ s$ where $\operatorname{pr}_{2}: M \times[0, \infty) \rightarrow[0, \infty)$ is the projection to the second coordinate. Restricting the structure maps of $\mathbb{T}_{H} M$ to $t \geq 0$, it is clear that the groupoids $\theta^{-1}\{t\}$ are the pair groupoid of $M$ for $t>0$ and $T_{H} M$ for $t=0$.

As all fibres of $T_{H} M$ are nilpotent Lie groups, thus amenable, it follows from ADR00 5.3.4] that $T_{H} M$ is amenable. The pair groupoid of $M$ is amenable as well.

\subsection{The zoom action}

The following zoom action of $\mathbb{R}_{>0}$ on $\mathbb{T}_{H} M$ by Lie groupoid automorphisms was defined in [vEY19, Def. 17] and [SH18, 5.3]. It plays an essential role for the definition of the pseudodifferential calculus in [vEY19. We will use it to construct a generalized fixed point algebra.

Definition 5.8. The zoom action of $\mathbb{R}_{>0}$ on $\mathbb{T}_{H} M$ is defined for $\lambda>0$ by

$$
\begin{array}{ll}
\alpha_{\lambda}(x, y, t)=\left(x, y, \lambda^{-1} t\right) & \text { for }(x, y, t) \in M \times M \times(0, \infty), \\
\alpha_{\lambda}(x, \xi, 0)=\left(x, \delta_{\lambda}(\xi), 0\right) & \text { for }(x, \xi) \in T_{H} M .
\end{array}
$$

Lemma 5.9. The zoom action of $\mathbb{R}_{>0}$ on the tangent groupoid of a filtered manifold $(M, H)$ is a smooth action by Lie groupoid automorphisms.

Proof. It can be checked easily that all $\alpha_{\lambda}$ are groupoid morphisms with underlying maps of the unit space

$$
M \times[0, \infty) \rightarrow M \times[0, \infty), \quad(x, t) \mapsto\left(x, \lambda^{-1} t\right) .
$$

They satisfy $\alpha_{\lambda \mu}=\alpha_{\lambda} \circ \alpha_{\mu}$ for all $\lambda, \mu>0$ and $\alpha_{1}=$ id. The smoothness on $M \times M \times(0, \infty)$ is clear. Let $\kappa: V \rightarrow U$ be an $H$-chart for $M$ and $\phi_{\kappa}: \mathbb{V} \rightarrow \mathbb{U}$ the corresponding chart for $\mathbb{T}_{H} M$. Then one computes for $\lambda>0$ and $(x, v, t) \in \mathbb{U}$

$$
\beta_{\lambda}(x, v, t):=\left(\phi_{\kappa} \circ \alpha_{\lambda} \circ \phi_{\kappa}^{-1}\right)(x, v, t)=\left(x, \lambda \cdot v, \lambda^{-1} t\right),
$$

where $\lambda \cdot\left(v_{1}, \ldots, v_{n}\right)=\left(\lambda^{q_{1}} v_{1}, \ldots, \lambda^{q_{n}} v_{n}\right)$. Hence, the action is smooth.

It will be useful to extend the notion of homogeneous quasi-norms on the osculating groups to $\mathbb{U}$ for $H$-charts $V \rightarrow U$. Let $\|\cdot\|: \mathbb{U} \rightarrow \mathbb{R}_{\geq 0}$ be given by

$$
\|(x, v, t)\|=\sum_{j=1}^{n}\left|v_{j}\right|^{1 / q_{j}} .
$$


It satisfies $\left\|\beta_{\lambda}(\gamma)\right\|=\lambda\|\gamma\|$ for all $\lambda>0$ and $\gamma \in \mathbb{U}$, where $\beta$ is the zoom action in coordinates as in (19). As opposed to homogeneous quasi-norms on homogeneous groups, $\left\|\gamma^{-1}\right\| \neq\|\gamma\|$ is possible for $\gamma \in \mathbb{U}$.

\subsection{Functoriality}

For a filtered manifold map $f:\left(M_{1}, H_{1}\right) \rightarrow\left(M_{2}, H_{2}\right)$, we already saw in Proposition 4.16 that it induces a Lie groupoid morphism $T f: T_{H_{1}} M_{1} \rightarrow T_{H_{2}} M_{2}$. It can be extended to a map of the corresponding tangent groupoids $\mathbb{E}: \mathbb{T}_{H_{1}} M_{1} \rightarrow \mathbb{T}_{H_{2}} M_{2}$ with

$$
\begin{aligned}
& \mathbb{E}(x, y, t)=(f(x), f(y), t) \quad \text { for }(x, y, t) \in M_{1} \times M_{1} \times(0, \infty), \\
& \mathbb{E}(x, \xi, 0)=\left(f(x), T_{x} f(\xi), 0\right) \quad \text { for }(x, \xi) \in T_{H_{1}} M_{1} \text {. }
\end{aligned}
$$

Note that the induced map $\mathbb{E}$ is equivariant for the respective zoom actions.

Proposition 5.10 ( $\mathbf{C P 1 9 b} 9.17,9.18])$. Let $f:\left(M_{1}, H_{1}\right) \rightarrow\left(M_{2}, H_{2}\right)$ be a filtered manifold map. Then $\mathbb{E}: \mathbb{T}_{H_{1}} M_{1} \rightarrow \mathbb{T}_{H_{2}} M_{2}$ is a Lie groupoid homomorphism with underlying map $(x, t) \mapsto(f(x), t)$ of the unit spaces.

The assignments $(M, H) \mapsto \mathbb{T}_{H} M$ and $f \mapsto \mathbb{E}$ define a functor from the category of filtered manifolds to the category of Lie groupoids. 


\section{CHAPTER 6}

\section{Convolution algebras for the tangent groupoid}

To apply the generalized fixed point algebra construction to the zoom action, we need to attach a $\mathrm{C}^{*}$-algebra to the tangent groupoid. In this chapter we recall the construction of groupoid $\mathrm{C}^{*}$-algebras. Furthermore, we define a certain Schwartz type algebra for the tangent groupoid by adapting CR08 to the filtered manifold setting. This algebra will be convenient to obtain a relatively continuous and complete subspace for the $\mathbb{R}_{>0}$-action.

\subsection{Haar system}

As $\mathbb{T}_{H} M$ is a Lie groupoid, it admits a smooth left Haar system (see for example Pat99, 2.3.1]). In the following, we explicitly describe a left Haar system.

Fix an atlas of $H$-charts $\left(\kappa_{i}: M \supseteq V_{i} \rightarrow U_{i} \subseteq \mathbb{R}^{n}\right)_{i \in I}$ for $M$. Let $\left(\rho_{i}\right)_{i \in I}$ be a partition of unity which is subordinate to the open cover $\left(V_{i}\right)_{i \in I}$ of $M$. One can define a measure $\nu$ on $M$ by setting

$$
\int_{M} f \mathrm{~d} \nu=\sum_{i \in I} \int_{U_{i}}\left(f \cdot \rho_{i}\right)\left(\kappa_{i}^{-1}(x)\right) \mathrm{d} x \quad \text { for } f \in \mathrm{C}_{c}(M),
$$

where $\mathrm{d} x$ denotes the Lebesgue measure on $U_{i} \subseteq \mathbb{R}^{n}$. Furthermore, the atlas of $H$-charts gives rise to a smooth family of measures on the osculating groups. Each $H$-chart $\kappa: V \rightarrow U$ induces a local trivialisation

$$
T \kappa:\left.T_{H} M\right|_{V} \cong \mathbb{R}^{n} \text {. }
$$

The Lebesgue measure on $\mathbb{R}^{n}$ can be pulled back using the graded isomorphism

$$
T_{x} \kappa: G(x) \stackrel{\cong}{\rightarrow} \mathbb{R}^{n} \quad \text { for all } x \in V .
$$

Recall that $d_{H}$ denotes the homogeneous dimension of $M$. Define for $f \in \mathrm{C}_{c}\left(\mathbb{T}_{H} M\right)$

$$
\begin{aligned}
\int f \mathrm{~d} \nu^{(x, 0)}:=\sum_{i \in I} \int_{\mathbb{R}^{n}} \rho_{i}(x) f\left(x,\left(T_{x} \kappa_{i}\right)^{-1}(v), 0\right) \mathrm{d} v & & \text { for } x \in M, \\
\int f \mathrm{~d} \nu^{(x, t)}:=t^{-d_{H}} \int_{M} f(x, y, t) \mathrm{d} \nu(y) & & \text { for } x \in M, t>0 .
\end{aligned}
$$

Lemma 6.1. The family of measures $\left\{\nu^{(x, t)}\right\}_{(x, t) \in M \times[0, \infty)}$ defines a smooth left Haar system on $\mathbb{T}_{H} M$.

Proof. For $(x, t) \in M \times[0, \infty)$ the support of $\nu^{(x, t)}$ is contained in $\mathbb{T}_{H} M^{(x, t)}$. The left invariance follows for $t>0$ as in the pair groupoid case. For $t=0$, this is due to the fact that the Lebesgue measure induces a bi-invariant Haar measure on the osculating groups. For $f \in \mathrm{C}_{c}^{\infty}\left(\mathbb{T}_{H} M\right)$, we show that the map

$$
(x, t) \rightarrow \int f \mathrm{~d} \nu^{(x, t)}
$$

is smooth. Using the partition of unity, $f$ can be written as a finite sum

$$
f=f_{\infty}+\sum_{i \in I} f_{i}
$$


with $f_{i} \in \mathrm{C}_{c}^{\infty}\left(\mathbb{V}_{i}\right)$ for $i \in I$ and $i=\infty$. As smoothness for $t>0$ is clear, it suffices to prove for all $H$-charts $\kappa: V \rightarrow U$ and $f \in \mathrm{C}_{c}^{\infty}(\mathbb{U})$ that

$$
\begin{aligned}
& (x, t) \mapsto t^{-d_{H}} \int_{U}\left(f \circ \phi_{\kappa}\right)\left(x, \kappa^{-1}(y), t\right) \mathrm{d} y \quad \text { for } t>0, \\
& (x, 0) \mapsto \int_{\mathbb{R}^{n}}\left(f \circ \phi_{\kappa}\right)\left(x,\left(T_{x} \kappa\right)^{-1}(v), 0\right) \mathrm{d} v=\int_{\mathbb{R}^{n}} f(\kappa(x), v, 0) \mathrm{d} v,
\end{aligned}
$$

is smooth. For $x \in V$ and $t>0$ consider the diffeomorphism

$$
\begin{aligned}
\tilde{\phi}_{\kappa}^{(x, t)}: U & \rightarrow \mathbb{U}^{(x, t)}:=\left\{v \in \mathbb{R}^{n} \mid\left(\varepsilon_{\kappa(x)}^{\kappa}\right)^{-1}(t \cdot v) \in U\right\}, \\
y & \mapsto t^{-1} \cdot \varepsilon_{\kappa(x)}^{\kappa}(y) .
\end{aligned}
$$

Because $\varepsilon_{\kappa(x)}^{\kappa}=\hat{\varepsilon}_{\kappa(x)}^{\kappa} \circ A_{\kappa(x)}$ as in (18) and the differential $d\left(\hat{\varepsilon}_{\kappa(x)}^{\kappa}\right)(y)$ is of upper triangular form with ones on the diagonal and $A_{k(x)}(y)=\left(B^{X}(\kappa(x))^{t}\right)^{-1}(y-\kappa(x))$, it follows that $\left|\operatorname{det}\left(d \tilde{\phi}_{\kappa}^{(x, t)}(y)\right)\right|=t^{-d_{H}}$ for all $y \in U$. Therefore, we obtain

$$
\begin{aligned}
t^{-d_{H}} \int_{U}\left(f \circ \phi_{\kappa}\right)\left(x, \kappa^{-1}(y), t\right) \mathrm{d} y & =t^{-d_{H}} \int_{U} f\left(\kappa(x), t^{-1} \cdot \varepsilon_{\kappa(x)}^{\kappa}(y), t\right) \mathrm{d} y \\
& =\int_{U^{\prime}(x, t)} f(\kappa(x), v, t) \mathrm{d} v .
\end{aligned}
$$

Thus the Haar measure is smooth.

\subsection{The groupoid $\mathrm{C}^{*}$-algebra}

Using the left Haar system $\left\{\nu^{(x, t)}\right\}_{(x, t) \in M \times[0, \infty)}$, the linear space $\mathrm{C}_{c}\left(\mathbb{T}_{H} M\right)$ can be equipped with the following involution and convolution:

$$
\begin{aligned}
f^{*}(\gamma) & =\overline{f\left(\gamma^{-1}\right)} \\
(f * g)(\gamma) & =\int f(\gamma \eta) g\left(\eta^{-1}\right) \mathrm{d} \nu^{s(\gamma)}(\eta)=\int f(\eta) g\left(\eta^{-1} \gamma\right) \mathrm{d} \nu^{r(\gamma)}(\eta)
\end{aligned}
$$

for $f, g \in \mathrm{C}_{c}\left(\mathbb{T}_{H} M\right)$ and $\gamma \in \mathbb{T}_{H} M$. More explicitly, the involution is given by

$$
f^{*}(x, y, t)=\overline{f(y, x, t)} \quad \text { for } t>0, \quad f^{*}(x, \xi, 0)=\overline{f\left(x, \xi^{-1}, 0\right)} .
$$

The convolution can be written as

$$
\begin{aligned}
& (f * g)(x, y, t)=t^{-d_{H}} \int_{M} f(x, z, t) g(z, y, t) \mathrm{d} \nu(z) \text { for } t>0, \\
& (f * g)(x, \xi, 0)=\int f(x, \eta, 0) g\left(x, \eta^{-1} \xi, 0\right) \mathrm{d} \nu^{(x, 0)}(x, \eta, 0) .
\end{aligned}
$$

Let the $I$-norm on $\mathrm{C}_{c}\left(\mathbb{T}_{H} M\right)$ be given by $\|f\|_{I}=\max \left\{\|f\|_{I, r},\|f\|_{I, s}\right\}$, where

$$
\|f\|_{I, r}=\sup _{(x, t)} \int|f| \mathrm{d} \nu^{(x, t)}
$$

and $\|f\|_{I, s}=\left\|f^{*}\right\|_{I, r}$. The (full) groupoid $\mathrm{C}^{*}$-algebra of $\mathbb{T}_{H} M$ is defined as the $\mathrm{C}^{*}$-completion of $\mathrm{C}_{c}\left(\mathbb{T}_{H} M\right)$ with respect to representations that are bounded by the $I$-norm as in Ren80, II,1.12].

Example 6.2. Let $G$ be a graded Lie group. By the description of $\mathbb{T}_{H} G$ as a transformation groupoid in Example 5.4. $\mathrm{C}^{*}\left(\mathbb{\mathbb { T }}_{H} G\right)$ is isomorphic to the crossed product $\mathrm{C}^{*}\left(G, \mathrm{C}_{0}(G \times[0, \infty))\right.$ (see $\left.\left.\operatorname{Ren} 80\right]\right)$. 


\subsection{Continuous field structure}

As the tangent groupoid $\mathbb{T}_{H} M$ is a continuous field of amenable groupoids, its $\mathrm{C}^{*}$-algebra admits a continuous field structure. The same is true for the bundle of osculating groups $T_{H} M$ viewed as a continuous bundle of groups over $M$.

Proposition 6.3. The $\mathrm{C}^{*}$-algebra of the tangent groupoid $\mathbb{T}_{H} M$ is a continuous field of $\mathrm{C}^{*}$-algebras over $[0, \infty)$ with fibres isomorphic to $\mathrm{C}^{*}\left(T_{H} M\right)$ for $t=0$ and the $\mathrm{C}^{*}$-algebra of compact operators $\mathbb{K}\left(L^{2} M\right)$ for $t>0$.

Proof. Recall that $\mathbb{T}_{H} M$ is a continuous field of groupoids by Lemma 5.7 As all groupoids $\theta^{-1}\{t\}$ are amenable, $\mathrm{C}^{*}\left(\mathbb{T}_{H} M\right)$ defines a continuous field of $\mathrm{C}^{*}$-algebras with fibres $\mathrm{C}^{*}\left(\theta^{-1}\{t\}\right)$ by LR01, 5.6]. For $t>0$ the groupoid $\theta^{-1}\{t\}$ is isomorphic to the pair groupoid of $M$. The Haar measure on $\theta^{-1}\{t\}$ is given by

$$
\int K(\gamma) \mathrm{d} \mu_{t}^{x}(\gamma)=t^{-d_{H}} \int_{M} K(x, y) \mathrm{d} \nu(y) \quad \text { for } K \in \mathrm{C}_{c}(M \times M) .
$$

There is a well-known isomorphism $\Phi_{t}: \mathrm{C}^{*}\left(M \times M, \mu_{t}\right) \rightarrow \mathbb{K}\left(L^{2} M\right)$ with

$$
\left(\Phi_{t}(K) \psi\right)(x)=t^{-d_{H}} \int_{M} K(x, y) \psi(y) \mathrm{d} \nu(y)
$$

for $K \in \mathrm{C}_{c}(M \times M)$ and $\psi \in \mathrm{C}_{c}(M)$. Hence, for $t>0$ we obtain epimorphisms $p_{t}: \mathrm{C}^{*}\left(\mathbb{T}_{H} M\right) \rightarrow \mathbb{K}\left(L^{2} M\right)$ given by

$$
\left(p_{t}(f) \psi\right)(x)=t^{-d_{H}} \int_{M} f(x, y, t) \psi(y) \mathrm{d} \nu(y)
$$

for $f \in \mathrm{C}_{c}\left(\mathbb{T}_{H} M\right), \psi \in \mathrm{C}_{c}(M)$ and $x \in M$.

Now consider the $\mathrm{C}^{*}$-algebra of the osculating groupoid at $t=0$.

Lemma 6.4. The $\mathrm{C}^{*}$-algebra $\mathrm{C}^{*}\left(T_{H} M\right)$ is a continuous field of $\mathrm{C}^{*}$-algebras over $M$ with fibre projections

$$
q_{x}: \mathrm{C}^{*}\left(T_{H} M\right) \rightarrow \mathrm{C}^{*}(G(x)) \quad \text { for } x \in M .
$$

Proof. As $T_{H} M$ is a continuous field of amenable groups over $M$, LR01, 5.6] applies again. Therefore, $\mathrm{C}^{*}\left(T_{H} M\right)$ is a continuous field of $\mathrm{C}^{*}$-algebras, where the fibre over $x \in M$ is isomorphic to the group $\mathrm{C}^{*}$-algebra of the osculating group $G(x)$.

Lemma 6.5. Denote by $p_{0}: \mathrm{C}^{*}\left(\mathbb{T}_{H} M\right) \rightarrow \mathrm{C}^{*}\left(T_{H} M\right)$ the ${ }^{*}$-homomorphism induced by restricting to $t=0$. There is a short exact sequence

$$
\mathrm{C}_{0}\left(\mathbb{R}_{>0}\right) \otimes \mathbb{K}\left(L^{2} M\right) \longleftrightarrow \mathrm{C}^{*}\left(\mathbb{T}_{H} M\right) \stackrel{p_{0}}{\longrightarrow} \mathrm{C}^{*}\left(T_{H} M\right) .
$$

Proof. The subset $M \times(0, \infty) \subset M \times[0, \infty)$ is open and invariant. By [HS87, the kernel of $p_{0}$ is $\mathrm{C}^{*}\left(\left.\mathbb{T}_{H} M\right|_{M \times(0, \infty)}\right)$. The fibre projections $p_{t}$ from 23 for $t>0$ combine to an isomorphism

$$
p: \mathrm{C}^{*}\left(\left.\mathbb{T}_{H} M\right|_{M \times(0, \infty)}\right) \rightarrow \mathrm{C}_{0}\left(\mathbb{R}_{>0}, \mathbb{K}\left(L^{2} M\right)\right),
$$

which is induced by $p(f)(t)=p_{t}(f)$ for $f \in \mathrm{C}_{c}\left(\left.\mathbb{T}_{H} M\right|_{M \times(0, \infty)}\right)$.

Remark 6.6. If the filtration is of step $r=1$, the $\mathrm{C}^{*}$-algebra $\mathrm{C}^{*}\left(T_{H} M\right)$ is isomorphic to $\mathrm{C}_{0}\left(T^{*} M\right)$. Namely, the fibrewise Fourier transform yields an isomorphism $\mathrm{C}^{*}(T M) \rightarrow \mathrm{C}_{0}\left(T^{*} M\right)$. If the osculating groups are not Abelian, $\mathrm{C}^{*}\left(T_{H} M\right)$ is noncommutative. 


\subsection{A Schwartz type *-algebra}

The goal of this section is to define a ${ }^{*}$-subalgebra $\mathcal{A}\left(\mathbb{T}_{H} M\right) \subset \mathrm{C}^{*}\left(\mathbb{T}_{H} M\right)$ consisting of $f \in \mathrm{C}^{\infty}\left(\mathbb{T}_{H} M\right)$ such that restricting to each $t>0$ gives a compactly supported function $f_{t} \in \mathrm{C}_{c}^{\infty}(M \times M)$, whereas $f_{0}$ has rapid decay in the fibres of $T_{H} M$. This follows the construction of the Schwartz type algebra in [CR08 for deformations to the normal cone.

First, the Schwartz type algebra will be defined locally as in CR08, 4.1] using the charts $\phi_{\kappa}: \mathbb{V} \rightarrow \mathbb{U}$ of $\mathbb{T}_{H} M$ obtained from $H$-charts $\kappa: V \rightarrow U$. We use the homogeneous quasi-norm on $\mathbb{U}$ from $(20)$. Consider the continuous function

$$
k: U \times \mathbb{R}^{n} \times[0, \infty) \rightarrow U \times \mathbb{R}^{n}
$$

given by $k(x, v, t)=(x, t \cdot v)$. Recall that $\theta: \mathbb{T}_{H} M \rightarrow[0, \infty)$ denotes the projection.

Definition 6.7. Let $\mathcal{A}(\mathbb{U})$ consist of all functions $f \in \mathrm{C}^{\infty}(\mathbb{U})$ satisfying

(i) there is $T>0$ and a compact subset $K \subset k(\mathbb{U})$ such that $(k(\gamma), \theta(\gamma)) \notin$ $K \times[0, T]$ implies $f(\gamma)=0$,

(ii) for all $p \in \mathbb{N}_{0}$ and $\alpha=\left(\alpha_{1}, \alpha_{2}, \alpha_{3}\right) \in \mathbb{N}_{0}^{n} \times \mathbb{N}_{0}^{n} \times \mathbb{N}_{0}$ there is a constant $D_{p, \alpha}>0$ such that

$$
\sup _{\gamma \in \mathbb{U}}(1+\|\gamma\|)^{p}\left|\partial_{x}^{\alpha_{1}} \partial_{v}^{\alpha_{2}} \partial_{t}^{\alpha_{3}} f(\gamma)\right| \leq D_{p, \alpha} .
$$

We check first that this space is invariant under Carnot diffeomorphisms.

Proposition 6.8 (|CR08, 4.2]). Let $F: U_{1} \rightarrow U_{2}$ be a Carnot diffeomorphism and $\mathbb{F}: \mathbb{U}_{1} \rightarrow \mathbb{U}_{2}$ the induced map as in Proposition 5.10 . Then $f \circ \mathbb{F} \in \mathcal{A}\left(\mathbb{U}_{1}\right)$ for all $f \in \mathcal{A}\left(\mathbb{U}_{2}\right)$.

Proof. As the induced map $\mathbb{F}: \mathbb{U}_{1} \rightarrow \mathbb{U}_{2}$ is smooth, it is clear that $f \circ \mathbb{F}$ is smooth for all $f \in \mathcal{A}\left(\mathbb{U}_{2}\right)$. In fact, as in the proof of [CP19b 9.15] we have

$$
\mathbb{F}(x, v)= \begin{cases}\left(F(x), t^{-1} \cdot\left(\varepsilon_{F(x)} \circ F \circ \varepsilon_{x}^{-1}\right)(t \cdot v), t\right) & \text { for } t>0, \\ \left(F(x), T_{x} F(v), 0\right) & \text { for } t=0,\end{cases}
$$

for $(x, v, t) \in \mathbb{U}_{1}$ and the respective $\varepsilon$-Carnot maps $U_{i} \times \mathbb{R}^{n} \rightarrow \mathbb{R}^{n}$ for $i=1,2$. To show condition (i) define $F_{k}: k_{1}\left(\mathbb{U}_{1}\right) \rightarrow k_{2}\left(\mathbb{U}_{2}\right)$ by

$$
(x, v) \mapsto\left(F(x),\left(\varepsilon_{F(x)} \circ F \circ \varepsilon_{x}^{-1}\right)(v)\right) .
$$

It is a diffeomorphism with inverse $\left(F^{-1}\right)_{k}$. The following diagram commutes

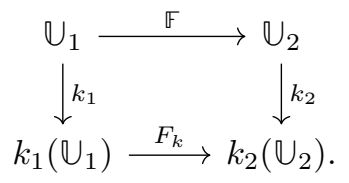

Let $K_{2} \subset k_{2}\left(\mathbb{U}_{2}\right)$ be a compact subset for $f$ as in (i). Then $K_{1}:=\left(F_{k}\right)^{-1}\left(K_{2}\right)$ is a compact subset such that $(f \circ \mathbb{F})(\gamma)=0$ if $k_{1}(\gamma) \notin K_{1}$. If $f$ vanishes for $t \geq T$, also $f \circ \mathbb{F}$ vanishes for $t \geq T$.

For the rapid decay property, write $\mathbb{E}(x, v, t)=(F(x), w(x, v, t), t)$. Because of this structure of $\mathbb{F}$, one can write for $\gamma=(x, v, t), \eta=\mathbb{F}(\gamma)$ and $\alpha \in \mathbb{N}_{0}^{n} \times \mathbb{N}_{0}^{n} \times \mathbb{N}_{0}$

$$
\partial_{\gamma}^{\alpha}(f \circ \mathbb{F})(\gamma)=\sum_{|\delta| \leq|\alpha|} \partial_{\eta}^{\delta} f(\eta) \cdot P_{\delta}(\gamma)
$$

where $P_{\delta}$ is a finite sum of products of the form

$$
\partial_{x}^{\delta_{1}} F_{i}(x) \cdot \partial_{\gamma}^{\delta_{2}} w_{j}(x, v, t)
$$


We only need to estimate each $P_{\delta}$ for $\gamma=(x, v, t)$ such that $k(x, v, t) \in K_{1}$ as otherwise $\partial_{\eta}^{\delta} f(\mathbb{F}(\gamma))=0$. In particular, $x$ is contained in a compact subset, so that $\partial_{x}^{\delta_{1}} F_{i}(x)$ is bounded. By $\left.\mathbf{C P 1 9 b}, 6.7\right]$,

$$
\varepsilon_{F(x)} \circ F \circ \varepsilon_{x}^{-1}(v)=T_{x} F(v)+O_{q}\left(\|v\|^{q+1}\right) .
$$

holds near $v=0$. Hence, Lemma 4.19 implies that there are smooth $R_{j, \alpha}$ for $j=1, \ldots, n$ such that

$$
w_{j}(x, v, t)=\left(T_{x} F(v)\right)_{j}+\sum_{|\alpha| \leq q_{j}+1 \leq[\alpha]} t^{[\alpha]-q_{j}} v^{\alpha} R_{j, \alpha}(x, t \cdot v) .
$$

For all $(x, v, t) \in k^{-1}\left(K_{1}\right)$ the components $x$ and $(x, t \cdot v)$ are in compact sets. Moreover, we only need to consider $t \leq T$. It follows that one can find $C_{\delta_{2}, j}>0$ and $m_{\delta_{2}, j}$ such that

$$
\left|\partial_{\gamma}^{\delta_{2}} w_{j}(\gamma)\right| \leq C_{\delta_{2}, j}(1+\|\gamma\|)^{m_{\delta_{2}, j}} \quad \text { for all } \gamma \in k^{-1}(K) \cap \theta^{-1}[0, T] .
$$

Together, this means that there are $C_{\delta}>0$ and $m_{\delta} \in \mathbb{N}$ such that $\left|P_{\delta}(\gamma)\right| \leq$ $C_{\delta}(1+\|\gamma\|)^{m_{\delta}}$ for all such $\gamma$. As $\mathbb{F}$ is a Carnot diffeomorphism, one can find likewise $D>0$ and $l>0$ such that for all $\gamma$ with $k(\gamma) \in K_{1}$

$$
1+\|\gamma\| \leq D(1+\|\mathbb{F}(\gamma)\|)^{l} .
$$

Let $p \in \mathbb{N}_{0}$. Because $f$ satisfies the rapid decay condition (ii) there are constants $D_{l\left(m_{\delta}+p\right), \delta}>0$ such that for all $\eta \in \mathbb{U}_{2}$

$$
\left|\partial_{\eta}^{\delta} f(\eta)\right| \leq D_{l\left(m_{\delta}+p\right), \delta}(1+\|\eta\|)^{-l\left(k+m_{\delta}\right)}
$$

It follows that $\sup _{\gamma \in \mathbb{U}}(1+\|\gamma\|)^{p}\left|\partial_{\gamma}^{\alpha} \mathbb{E}(\gamma)\right|<\infty$.

The invariance under Carnot diffeomorphisms allows to define the Schwartz type algebra in the following way. For an $H$-chart $\kappa: V \rightarrow U$ let

$$
\mathcal{A}(\mathbb{V}):=\left\{f \in \mathrm{C}^{\infty}(\mathbb{V}) \mid f \circ \phi_{\kappa}^{-1} \in \mathcal{A}(\mathbb{U})\right\}
$$

We will denote by $r_{1}, s_{1}: \mathbb{T}_{H} M \rightarrow M$ the maps given by $r_{1}=\mathrm{pr}_{1}$ or and $s_{1}=\operatorname{pr}_{1} \circ s$.

Definition 6.9 $(\overline{\text { CR08 }}, 4.4])$. The Schwartz type algebra $\mathcal{A}\left(\mathbb{T}_{H} M\right)$ is the space of functions $f \in \mathrm{C}^{\infty}\left(\mathbb{\mathbb { T }}_{H} M\right)$ such that

(i) there are $T>0$ and a compact subset $K \subset M \times M$ such that $\left(r_{1}, s_{1}, \theta\right)(\gamma) \notin$ $K \times[0, T]$ implies $f(\gamma)=0$,

(ii) $f$ has rapid decay at $t=0$, that is, for all $H$-charts $\kappa: V \rightarrow U$ and $\chi \in \mathrm{C}_{c}^{\infty}(V \times V \times[0, \infty))$, the function $f_{\chi}$ belongs to $\mathcal{A}(\mathbb{V})$, where

$$
f_{\chi}(\gamma)=\left(\chi \circ\left(r_{1}, s_{1}, \theta\right)\right)(\gamma) f(\gamma) \text { for } \gamma \in \mathbb{V} .
$$

We will verify later that $\mathcal{A}\left(\mathbb{T}_{H} M\right)$ is indeed an algebra.

Lemma 6.10 (CR08, (5)]). Let $\left(\kappa_{i}: V_{i} \rightarrow U_{i}\right)_{i \in I}$ be an atlas of $H$-charts for $M$. The space $\mathcal{A}\left(\mathbb{T}_{H} M\right)$ can be decomposed as

$$
\mathcal{A}\left(\mathbb{\mathbb { V }}_{H} M\right)=\sum_{i \in I} \mathcal{A}\left(\mathbb{V}_{i}\right)+\mathrm{C}_{c}^{\infty}\left(\mathbb{V}_{\infty}\right)
$$

Proof. Let $f \in \mathcal{A}(\mathbb{V})$ for an $H$-chart $\kappa: V \rightarrow U$. We claim that $f \in \mathcal{A}\left(\mathbb{T}_{H} M\right)$. There is a diffeomorphism $\kappa_{k}: V \times V \rightarrow k(\mathbb{U})$ given by

$$
(x, y) \mapsto\left(\kappa(x), \varepsilon_{\kappa(x)}^{\kappa}(\kappa(y))\right) .
$$


It makes the following diagram commute

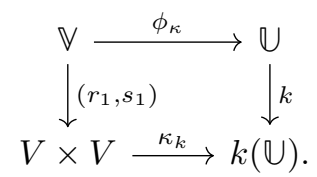

Let $K_{U} \subset k(\mathbb{U})$ be a compact subset for $f \circ \phi_{\kappa}^{-1}$ as in Definition 6.7. Then $\varphi^{-1}\left(K_{U}\right)$ is compact and $f(\gamma)=0$ whenever $\left(r_{1}, s_{1}\right)(\gamma) \notin \varphi^{-1}\left(K_{U}\right)$. In fact, condition (i) from Definition 6.9 for $f \in \mathrm{C}^{\infty}(\mathbb{V})$ is equivalent to (i) from Definition 6.7 for $f \circ \phi_{\kappa}^{-1} \in \mathrm{C}^{\infty}(\mathbb{U})$. Moreover, $f$ has rapid decay at $t=0$ by the invariance under Carnot diffeomorphisms from Proposition 6.8 Clearly, $\mathcal{A}\left(\mathbb{T}_{H} M\right)$ contains all smooth function with compact support in $M \times M \times \mathbb{R}_{>0}$ and is closed under finite sums.

For the converse inclusion, let $f \in \mathcal{A}\left(\mathbb{T}_{H} M\right)$ and let $K \subset M \times M$ be a compact set and $T>0$ as in (i) Note that $V_{i} \times V_{i} \times[0, \infty)$ for $i \in I$ and $M \times M \times(0, \infty)$ define an open cover of $K \times[0, T]$. Therefore, there is a finite partition of unity $\left(\rho_{i}\right)_{i \in I \cup\{\infty\}}$ consisting of smooth, compactly supported functions subordinate to this open cover. By (ii) $f_{i}:=f_{\rho_{i}}$ are in $\mathcal{A}\left(\mathbb{V}_{i}\right)$ for $i \in I$ and $f_{\infty} \in \mathrm{C}_{c}^{\infty}\left(\mathbb{V}_{\infty}\right)$. This yields a decomposition of $f$ as above.

For a vector bundle $E \rightarrow M$ consider functions that have uniform rapid decay in the fibres.

Definition 6.11 ([CR08, 4.6]). Let $\pi: E \rightarrow M$ be a smooth vector bundle. A function $f \in \mathrm{C}^{\infty}(E)$ has uniform rapid decay in the fibres, if for all local trivializations $\varphi:\left.E\right|_{V} \rightarrow V \times \mathbb{R}^{m}, p \in \mathbb{N}_{0}$ and $\alpha=\left(\alpha_{1}, \alpha_{2}\right) \in \mathbb{N}_{0}^{n} \times \mathbb{N}_{0}^{m}$ and all cutoff functions $\chi \in \mathrm{C}_{c}^{\infty}(V)$

$$
\sup _{(x, v) \in V \times \mathbb{R}^{m}}(1+|v|)^{p}\left|\partial_{x}^{\alpha_{1}} \partial_{v}^{\alpha_{2}} \chi(x) f\left(\varphi^{-1}(x, v)\right)\right|<\infty .
$$

Let $\mathcal{S}(E)$ be the space of functions with uniform rapid decay in the fibres. Let $\mathcal{S}_{\mathrm{cp}}(E)$ consist of all $f \in \mathcal{S}(E)$ such that $\pi(\operatorname{supp} f)$ is compact.

Lemma 6.12. The restrictions $e_{t}: f \mapsto f_{t}$ for $t \in[0, \infty)$ yield surjections

$$
\begin{aligned}
& e_{t}: \mathcal{A}\left(\mathbb{T}_{H} M\right) \rightarrow \mathrm{C}_{c}^{\infty}(M \times M) \quad \text { for } t>0, \\
& e_{0}: \mathcal{A}\left(\mathbb{T}_{H} M\right) \rightarrow \mathcal{S}_{\mathrm{cp}}\left(T_{H} M\right) .
\end{aligned}
$$

Proof. Let $f \in \mathcal{A}\left(\mathbb{T}_{H} M\right)$. Condition (i) ensures that $f_{t}$ is compactly supported for each $t>0$. For $t=0$ it implies compact support in the $M$-direction. Moreover, $f_{0}$ belongs to $\mathcal{S}_{\mathrm{cp}}(E)$ as any locally defined norm on the fibres of $T_{H} M$ is equivalent to the homogeneous quasi-norm.

For $t>0$, surjectivity is easily seen by extending a function in $\mathrm{C}_{c}^{\infty}(M \times M \times\{t\})$ smoothly to a function in $\mathrm{C}_{c}^{\infty}\left(M \times M \times \mathbb{R}_{>0}\right)$.

At $t=0$, it suffices to show that $e_{0}: \mathcal{A}(\mathbb{U}) \rightarrow \mathcal{S}_{\mathrm{cp}}\left(U \times \mathbb{R}^{n}\right)$ is surjective for each $H$-chart $\kappa: V \rightarrow U$. Let $f_{0} \in \mathcal{S}_{\mathrm{cp}}\left(U \times \mathbb{R}^{n}\right)$ and let $K_{0} \subset U$ be a compact subset such that $f(x, v)=0$ whenever $x \notin K_{0}$. As $K_{0}$ is compact there is a $1>\delta>0$ such that $\left(\varepsilon_{x}^{\kappa}\right)^{-1}(v) \in U$ for all $x \in K_{0}$ and $\Phi(v) \leq \delta$, where $\Phi$ is the smooth function defined in Remark 3.13 Choose a smooth function $0 \leq \chi \leq 1$ on $[0, \infty)$ which satisfies $\chi(0)=1$ and $\chi(t)=0$ whenever $t \geq \delta$. Define $f(x, v, t):=f_{0}(x, v) \chi(t) \chi(\Phi(t \cdot v))$. The following set is compact and contained in $k(\mathbb{U})$

$$
K:=\left\{(x, v) \in U \times \mathbb{R}^{n} \mid x \in K_{0} \text { and } \Phi(v) \leq \delta\right\} .
$$

Then $f(x, v, t)=0$ whenever $k(x, v, t) \notin K$ or $t \geq \delta$. Moreover, $f$ satisfies the rapid decay condition and $e_{0}(f)=f_{0}$. 
We proceed by showing that the Schwartz type algebra is a *-algebra with respect to the operations in (21) and $(22)$. First, we prove the following estimates for the groupoid inversion $i: \mathbb{U} \rightarrow \mathbb{U}$ and product $m: \mathbb{U}^{(2)} \rightarrow \mathbb{U}$ with respect to the homogeneous quasi-norm.

Lemma 6.13. Let $K \subset k(\mathbb{U})$ be compact, $T>0$ and $\alpha \in \mathbb{N}_{0}^{2 n+1}$.

(i) There are $C_{i, \alpha, K, T}>0$ and $l_{i, \alpha} \in \mathbb{N}$ such that for all $\gamma \in \mathbb{U}$ with $k(\gamma) \in K$ and $\theta(\gamma) \leq T$

$$
\left|\partial_{\gamma}^{\alpha} i(\gamma)_{j}\right| \leq C_{i, \alpha, K, T}(1+\|\gamma\|)^{l_{i, \alpha}}
$$

for $j=1, \ldots, 2 n+1$.

(ii) There are $C_{m, \alpha, K, T}>0$ and $l_{m, \alpha} \in \mathbb{N}$ such that for all $(\gamma, \eta) \in \mathbb{U}^{(2)}$ with $k(\gamma), k(\eta) \in K$ and $\theta(\gamma) \leq T$

$$
\left|\partial_{\gamma}^{\alpha} m(\gamma, \eta)_{j}\right| \leq C_{m, \alpha, K, T}(1+\|\gamma\|)^{l_{m, \alpha}}(1+\|\eta\|)^{l_{m, \alpha}}
$$

for $j=1, \ldots, 2 n+1$.

Proof. For (i) the inversion is given in local coordinates as $i: \mathbb{U} \rightarrow \mathbb{U}$ with

$$
i(x, v, t)= \begin{cases}\left(\varepsilon_{x}^{-1}(t \cdot v), t^{-1} \cdot \varepsilon_{\varepsilon_{x}^{-1}(t \cdot v)}(x), t\right) & \text { for } t>0, \\ (x,-v, 0) & \text { for } t=0\end{cases}
$$

for $(x, v, t) \in \mathbb{U}$ by $\mathbf{C P 1 9 b}$ 9.9]. As noted there, near $v=0$

$$
\varepsilon_{\varepsilon_{x}^{-1}(v)}(x)=-v+O_{q}\left(\|v\|^{q+1}\right)
$$

holds. So Lemma 4.19 and the compactness of $K \times[0, T]$ can be used, similarly as in the proof of Proposition 6.8, to derive bounds of the desired form.

We proceed similarly for (ii) and write for $\left((x, v, t),\left(\varepsilon_{x}^{-1}(t \cdot v), w, t\right)\right) \in \mathbb{U}^{(2)}$

$$
m\left((x, v, t),\left(\varepsilon_{x}^{-1}(t \cdot v), w, t\right)\right)= \begin{cases}\left(x, t^{-1} \cdot\left(\varepsilon_{x} \circ \varepsilon_{\varepsilon_{x}^{-1}(t \cdot v)}^{-1}\right)(t \cdot w), t\right) & \text { for } t>0 \\ (x, v \cdot w, 0) & \text { for } t=0\end{cases}
$$

as in $\mathbf{C P 1 9 b}, 9.11]$. By their argument, for all $x \in U$

$$
\varepsilon_{x} \circ \varepsilon_{\varepsilon_{x}^{-1}(v)}^{-1}(w)=v \cdot_{x} w+O\left(\|(v, w)\|^{q+1}\right)
$$

holds for $(v, w)$ near $(0,0)$. As $K \times[0, T]$ is compact and the group multiplication is polynomial and depends continuously on $x$, one obtains estimates of the claimed form using again Lemma 4.19 .

Corollary 6.14. For $K \subset k(\mathbb{U})$ compact and $T>0$ there are $C_{K, T}>0$ and $l \in \mathbb{N}$ such that

(i) for all $\gamma \in \mathbb{U}$ with $k(\gamma) \in K$ and $\theta(\gamma) \leq T$

$$
1+\left\|\gamma^{-1}\right\| \leq C_{K, T}(1+\|\gamma\|)^{l}
$$

(ii) for all $\gamma, \eta \in \mathbb{U}$ with $\left(\eta^{-1}, \gamma\right) \in \mathbb{U}^{(2)}, k(\eta), k\left(\eta^{-1} \cdot \gamma\right) \in K$ and $\theta(\eta) \leq T$

$$
1+\|\gamma\| \leq C_{K, T}(1+\|\eta\|)^{l}\left(1+\left\|\eta^{-1} \cdot \gamma\right\|\right)^{l} .
$$

THEOREM 6.15. The Schwartz type algebra $\mathcal{A}\left(\mathbb{T}_{H} M\right)$ is a ${ }^{*}$-algebra with respect to the involution and convolution defined in 21) and 22. Moreover, there are inclusions of *-algebras

$$
\mathrm{C}_{c}^{\infty}\left(\mathbb{T}_{H} M\right) \subset \mathcal{A}\left(\mathbb{T}_{H} M\right) \subset \mathrm{C}^{*}\left(\mathbb{T}_{H} M\right) .
$$


Proof. For the involution, note that if $K \subset M \times M$ is a compact subset for $f \in \mathcal{A}\left(\mathbb{T}_{H} M\right)$ as in Definition 6.9 $i(K) \subset M \times M$ is a compact subset for $f^{*}$. Here $i: M \times M \rightarrow M \times M$ is the inversion on the pair groupoid. By Lemma 6.10 and linearity, it suffices to show that $f^{*} \in \mathcal{A}\left(\mathbb{V}_{j}\right)$ for $f \in \mathcal{A}\left(\mathbb{V}_{j}\right)$ for $j \in I$. For $j=\infty$, it is clear that $f^{*}$ lies again in $\mathrm{C}_{c}^{\infty}\left(\mathbb{V}_{\infty}\right)$.

Suppose now that $f \in \mathcal{A}(\mathbb{U})$ for an $H$-chart $\kappa: V \rightarrow U$. Denote by $K_{f}, K_{f^{*}} \subset$ $k(\mathbb{U})$ the compact subsets for $f, f^{*}$ respectively. As $f^{*}(\gamma)=\overline{f \circ i(\gamma)}$, the derivatives of the inversion $i$ can be bounded by powers of $1+\|\gamma\|$ as in Lemma 6.13 with $K=K_{f^{*}}$ and $T$ such that $f$ vanishes for $t \geq T$. By Corollary 6.14 $1+\|\gamma\| \leq$ $C_{K_{f}, T}(1+\|i(\gamma)\|)^{l}$ holds for all $\gamma \in k^{-1}\left(K_{f}\right)$ with $\theta(t) \leq T$. Then the Schwartz seminorms for $f$ can be used to show that $f^{*}$ has the rapid decay property.

For the convolution, note that $f * g$ for $f, g \in \mathcal{A}\left(\mathbb{\mathbb { T }}_{H} M\right)$ is a well-defined smooth function as $f, g$ are Schwartz functions at $t=0$ and compactly supported otherwise. If $K_{f}, K_{g} \subset M \times M$ are compact subsets for $f, g$, it follows that $K:=m\left(K_{f}, K_{g}\right)$ is a compact subset for $f * g$, where $m$ denotes the multiplication in the pair groupoid.

To prove the rapid decay, we decompose $g$ as in Lemma 6.10 into a finite sum of $g_{j} \in \mathcal{A}\left(\mathbb{V}_{j}\right), j=1, \ldots, m$, and $g_{\infty} \in \mathrm{C}_{c}^{\infty}\left(\mathbb{V}_{\infty}\right)$ and use linearity to write

$$
f * g=\sum_{j \in\{1, \ldots, m, \infty\}} f * g_{j} .
$$

Let $T>0$ be such that $f$ and $g$ vanish for $t \geq T$. As there is a compact subset $K_{g_{j}} \subset V_{j} \times V_{j}$ for each $g_{j}$, it follows that

$$
K_{f * g_{j}} \times[0, T]=m\left(K_{f}, K_{g_{j}}\right) \times[0, T]
$$

is openly covered by $V_{j} \times V_{j} \times[0, \infty)$ and $M \times M \times(0, \infty)$ as for $t=0$ the source and range maps coincide. Let $\{\chi, 1-\chi\}$ be a corresponding compactly supported partition of unity. Let $t_{0}>0$ be such that $1-\chi$ vanishes for $t<t_{0}$ and choose a bump function $\omega \in \mathrm{C}^{\infty}\left(\mathbb{R}_{>0}\right)$ with $\omega(t)=1$ for $t \geq t_{0}$ and $\omega(t)=0$ for $t<t_{0} / 2$. Then we can write

$$
\left(f * g_{j}\right)_{(1-\chi)}=\left(f \cdot(\omega \circ \theta) * g_{j} \cdot(\omega \circ \theta)\right)_{(1-\chi)} .
$$

This is a convolution of functions in $\mathrm{C}_{c}^{\infty}\left(M \times M \times \mathbb{R}_{>0}\right)$, so that the result is clearly contained in $\mathcal{A}\left(\mathbb{T}_{H} M\right)$.

Consider now $\left(f * g_{j}\right)_{\chi}$. Let $\omega_{i} \in \mathrm{C}_{c}^{\infty}\left(V_{j}\right)$ for $i=1,2$ be such that $\omega_{1}(x)=1$ for all $x \in r_{1}(\operatorname{supp} \chi)$ and $\omega_{2}(x)=1$ for all $x \in r\left(K_{g_{j}}\right)$. For each $\gamma \in \mathbb{T}_{H} M$

$$
\begin{aligned}
\left(f * g_{j}\right)_{\chi}(\gamma) & =\chi \circ\left(r_{1}, s_{1}, \theta\right)(\gamma) \int f(\gamma \eta) g_{j}\left(\eta^{-1}\right) \mathrm{d}^{s(\gamma)}(\eta) \\
& =\chi \circ\left(r_{1}, s_{1}, \theta\right)(\gamma) \omega_{1}\left(r_{1}(\gamma)\right) \int f(\gamma \eta) \omega_{2}\left(r_{1}\left(\eta^{-1}\right)\right) g_{j}\left(\eta^{-1}\right) \mathrm{d}^{s(\gamma)}(\eta) \\
& \left.=\chi \circ\left(r_{1}, s_{1}, \theta\right)(\gamma) \int \omega_{1}\left(r_{1}(\gamma \eta)\right) f(\gamma \eta) \omega_{2}\left(s_{1}(\gamma \eta)\right)\right) g_{j}\left(\eta^{-1}\right) \mathrm{d}^{s(\gamma)}(\eta) \\
& =\left(f \cdot\left(\omega_{1} \circ r_{1}\right) \cdot\left(\omega_{2} \circ s_{1}\right) * g_{j}\right)_{\chi}
\end{aligned}
$$

holds. In conclusion, we obtained a finite decomposition

$$
f * g=\sum_{j=1}^{m}\left(f_{j} * g_{j}\right)_{\chi_{j}}+\sum_{j \in\{1, \ldots, m, \infty\}} f_{j}^{\infty} * g_{j}^{\infty}
$$

with $f_{j}^{\infty}, g_{j}^{\infty} \in \mathrm{C}_{c}^{\infty}\left(\mathbb{V}_{\infty}\right), f_{j}, g_{j} \in \mathcal{A}\left(\mathbb{V}_{j}\right)$ and $\chi_{j} \in \mathrm{C}_{c}^{\infty}\left(V_{j} \times V_{j} \times[0, \infty)\right)$. Therefore, it is left to show that $\mathcal{A}(\mathbb{U}) * \mathcal{A}(\mathbb{U}) \subseteq \mathcal{A}(\mathbb{U})$.

Let $f, g \in \mathcal{A}(\mathbb{U})$ and denote by $K_{f}, K_{g}, K_{f * g} \subseteq k(\mathbb{U})$ the respective compact subsets, and let $T>0$ be such that $f, g$ vanish for $t \geq T$. To show rapid decay, we 
must estimate the derivatives

$$
\partial_{\gamma}^{\alpha} g\left(\eta^{-1} \gamma\right)=\sum \partial_{\eta^{-1} \gamma}^{\delta} g\left(\eta^{-1} \gamma\right) \cdot M^{\delta}\left(\eta^{-1}, \gamma\right) \text { for } \eta^{-1} \gamma \in k^{-1}\left(K_{g}\right) \text { and } t<T
$$

where $M^{\delta}\left(\eta^{-1}, \gamma\right)$ is a product of $\partial_{\gamma}^{\delta_{i}} m_{j}\left(\eta^{-1}, \gamma\right)$. The bounds from Lemma 6.13 for the multiplication and inverse allow to estimate $\left|M^{\delta}\right|$ for all $\gamma \in k^{-1}\left(K_{f * g}\right)$ and $\eta \in k^{-1}\left(K_{f}\right)$ with $t<T$ by $C(1+\|\eta\|)^{r}(1+\|\gamma\|)^{s}$ for some $C>0$ and $r, s \in \mathbb{N}_{0}$. Therefore, we can use the rapid decay of $f$ and $g$ to estimate for $p \in \mathbb{N}$ using Corollary 6.14

$$
\begin{aligned}
& (1+\|\gamma\|)^{p} \int\left|f(\eta) \| \partial_{\eta^{-1} \gamma}^{\delta} g\left(\eta^{-1} \gamma\right) \cdot M^{\delta}\left(\eta^{-1}, \gamma\right)\right| \mathrm{d} \nu^{r(\gamma)}(\eta) \\
\lesssim & (1+\|\gamma\|)^{p+s} \int|f(\eta)|(1+\|\eta\|)^{r}\left|\partial_{\eta^{-1} \gamma}^{\delta} g\left(\eta^{-1} \gamma\right)\right| \mathrm{d} \nu^{r(\gamma)}(\eta) \\
\lesssim & (1+\|\gamma\|)^{p+s} \int|f(\eta)|(1+\|\eta\|)^{r}\left(1+\left\|\eta^{-1} \gamma\right\|\right)^{-l(p+s)} \mathrm{d} \nu^{r(\gamma)}(\eta) \\
\lesssim & \int|f(\eta)|(1+\|\eta\|)^{r+l(p+s)} \mathrm{d} \nu^{r(\gamma)}(\eta) \\
\lesssim & \int(1+\|\eta\|)^{-d_{H}-1} \mathrm{~d} \nu^{r(\gamma)}(\eta)<\infty
\end{aligned}
$$

for all $\gamma \in \mathbb{U}$. This finishes the proof that $\mathcal{A}\left(\mathbb{T}_{H} M\right)$ is a ${ }^{*}$-algebra.

Clearly, $\mathrm{C}_{c}^{\infty}\left(\mathbb{T}_{H} M\right)$ is contained in $\mathcal{A}\left(\mathbb{T}_{H} M\right)$. For $f \in \mathcal{A}(\mathbb{U})$, we can construct a sequence $f_{m} \in \mathrm{C}_{c}^{\infty}(\mathbb{U})$ which converges to $f$ in the $I$-norm. This will imply that $\mathcal{A}\left(\mathbb{\mathbb { T }}_{H} M\right) \subset \mathrm{C}^{*}\left(\mathbb{\mathbb { T }}_{H} M\right)$. This can be done by choosing a sequence of functions $\chi_{m} \in \mathrm{C}_{c}^{\infty}\left(\mathbb{R}^{n}\right)$ with $0 \leq \chi_{m} \leq 1, \operatorname{supp}\left(\chi_{m}\right) \subset B(0, m)$ and $\left.\chi_{m}\right|_{B(0, m-1)} \equiv 1$. Then $f_{m}(x, v, t):=f(x, v, t) \chi_{m}(v)$ is such a sequence.

\subsection{The zoom action on the convolution algebras}

The zoom action on the tangent groupoid, introduced in Definition 5.8 , induces an action on the convolution algebras.

Lemma 6.16. The maps $\sigma_{\lambda}: \mathrm{C}_{c}\left(\mathbb{T}_{H} M\right) \rightarrow \mathrm{C}_{c}\left(\mathbb{T}_{H} M\right)$ defined by

$$
\left(\sigma_{\lambda} f\right)(\gamma)=\lambda^{d_{H}} f\left(\alpha_{\lambda}(\gamma)\right) \text { for } \lambda>0 \text { and } f \in \mathrm{C}_{c}\left(\mathbb{T}_{H} M\right)
$$

extend to a strongly continuous $\mathbb{R}_{>0}$-action on $\mathrm{C}^{*}\left(\mathbb{T}_{H} M\right)$. Moreover, $\mathcal{A}\left(\mathbb{T}_{H} M\right)$ is invariant under the action.

Proof. Note that the Haar system $\left\{\nu^{(x, t)}\right\}_{(x, t) \in M \times[0, \infty)}$ satisfies

$$
\int \sigma_{\lambda} f \mathrm{~d} \nu^{(x, t)}=\int f \mathrm{~d} \nu^{\left(x, \lambda^{-1} t\right)} \quad \text { for all } f \in \mathrm{C}_{c}\left(\mathbb{T}_{H} M\right) .
$$

Using this one can show $\sigma_{\lambda}(f * g)=\sigma_{\lambda}(f) * \sigma_{\lambda}(g)$ for $f, g \in \mathrm{C}_{c}\left(\mathbb{\mathbb { T }}_{H} M\right)$. Furthermore, all $\sigma_{\lambda}$ are linear and satisfy $\sigma_{\lambda}\left(f^{*}\right)=\left(\sigma_{\lambda}(f)\right)^{*}$ for all $f \in \mathrm{C}_{c}\left(\mathbb{T}_{H} M\right)$. As each $\sigma_{\lambda}$ is an isometry with respect to the $I$-norm, it follows that $\sigma$ extends to a strongly continuous action on $\mathrm{C}^{*}\left(\mathbb{T}_{H} M\right)$.

To see that $\mathcal{A}\left(\mathbb{T}_{H} M\right)$ is invariant, note that it suffices to show this for $f \in \mathcal{A}(\mathbb{U})$, as $\mathrm{C}_{c}^{\infty}\left(M \times M \times \mathbb{R}_{>0}\right)$ is invariant. Because $k\left(\beta_{\lambda}(x, v, t)\right)=(x, t \cdot v)=k(x, v, t)$ for all $\lambda>0$, one can take the compact set $K_{f}$ for $f$ for all $\sigma_{\lambda}(f)$. Furthermore, for fixed $\lambda>0$ the function $\sigma_{\lambda}(f)$ has compact support in the $t$-direction and satisfies the rapid decay condition. This follows from the homogeneity of the quasi-norm.

Lemma 6.17. The ideal $\operatorname{ker}\left(p_{0}\right) \triangleleft \mathrm{C}^{*}\left(\mathbb{T}_{H} M\right)$ is invariant under the zoom action. Under the isomorphism from 25

$$
p: \operatorname{ker}\left(p_{0}\right) \rightarrow \mathrm{C}_{0}\left(\mathbb{R}_{>0}\right) \otimes \mathbb{K}\left(L^{2} M\right)
$$


the zoom action corresponds to the action $\tau \otimes 1$, where $\tau$ is induced by the free and proper scaling action of $\mathbb{R}_{>0}$ on itself, namely,

$$
\left(\tau_{\lambda} f\right)(t)=f\left(\lambda^{-1} t\right) \quad \text { for } f \in \mathrm{C}_{0}\left(\mathbb{R}_{>0}\right) \text { and } \lambda, t>0 .
$$

Proof. The homomorphism $p_{0}$ is equivariant for the zoom action and the action on $\mathrm{C}^{*}\left(T_{H} M\right)$ induced by the dilations in Definition 4.12 The second claim follows from the computation that $p \circ \sigma_{\lambda}=\left(\tau_{\lambda} \otimes 1\right) \circ p$ for all $\lambda>0$. 


\section{CHAPTER 7}

\section{The fixed point algebra construction for filtered manifolds}

For an ordinary manifold $M$, the $\mathrm{C}^{*}$-algebra generated by the principal symbols with compact support in the $M$-direction are the continuous functions on the cosphere bundle $\mathrm{C}_{0}\left(S^{*} M\right)$. This algebra is the generalized fixed point algebra of the action of $\mathbb{R}_{>0}$ on $\mathrm{C}_{0}\left(T^{*} M \backslash(M \times\{0\})\right)$ which is induced by the scalings

$$
\lambda \cdot(x, \xi)=\left(x, \lambda^{-1} \xi\right) \quad \text { for } x \in M, \xi \in T_{x}^{*} M .
$$

Here, it is essential to take out the zero section of the cotangent bundle as otherwise the action is not proper.

In the case of a filtered manifold, we will not apply Fourier transform on $T_{H} M$ as the osculating groups are not necessarily Abelian. Therefore, we trace back what $\mathrm{C}_{0}\left(T^{*} M \backslash(M \times\{0\})\right)$ corresponds to under inverse Fourier transform. Let $\widehat{f}$ denote the Euclidean Fourier transform of $f \in \mathcal{S}\left(\mathbb{R}^{n}\right)$. Recall that the trivial representation of the group $\mathbb{R}^{n}$ induces a representation $\widehat{\pi}_{\text {triv }}: C^{*}\left(\mathbb{R}^{n}\right) \rightarrow \mathbb{C}$ as in $(11)$. Then

$$
\widehat{f}(0)=0 \Longleftrightarrow \int_{\mathbb{R}^{n}} f(x) \mathrm{d} x=0 \Longleftrightarrow f \in \operatorname{ker}\left(\widehat{\pi}_{\text {triv }}\right) .
$$

This observation motivates the following definition of an analogue of the ideal $\mathrm{C}_{0}\left(T^{*} M \backslash(M \times\{0\})\right)$ for filtered manifolds.

\subsection{The ideals $\sqrt{ }$ and $\mathbb{I}_{0}$}

Definition 7.1. Let $\mathbb{J}_{0}$ be the ideal in $\mathrm{C}^{*}\left(T_{H} M\right)$ defined as

$$
\mathbb{J}_{0}=\bigcap_{x \in M} \operatorname{ker}\left(\widehat{\pi}_{\text {triv }} \circ q_{x}\right)
$$

Here, $\widehat{\pi}_{\text {triv }}: \mathrm{C}^{*}(G(x)) \rightarrow \mathbb{C}$ denotes the representation induced by the trivial representation of the osculating group $G(x)$.

The ideal $\mathbb{J}_{0}$ can be extended to an ideal $\mathbb{l}$ in $\mathrm{C}^{*}\left(\mathbb{T}_{H} M\right)$ as follows.

Definition 7.2. Let $\mathbb{J}$ denote the ideal in $\mathrm{C}^{*}\left(\mathbb{T}_{H} M\right)$ given by

$$
\rrbracket=\bigcap_{x \in M} \operatorname{ker}\left(\widehat{\pi}_{\text {triv }} \circ q_{x} \circ p_{0}\right)
$$

Both ideals $\rrbracket$ and $\rrbracket_{0}$ are invariant under the zoom action $\sigma$ of $\mathbb{R}_{>0}$. In the following, the generalized fixed point algebra construction will be applied to this $\mathbb{R}_{>0}$-action on $\mathbb{J}$. In order to do so, consider the following ${ }^{*}$-subalgebra of $\mathbb{J}$.

Definition 7.3. Let $\mathcal{R} \subset \rrbracket$ consist of all $f \in \mathcal{A}\left(\mathbb{T}_{H} M\right)$ such that

$$
\int f \mathrm{~d} \nu^{(x, 0)}=0 \quad \text { for all } x \in M
$$




\subsection{Proof of continuous square-integrability}

We show first the following lemma, which will replace an application of the mean value theorem in the Euclidean case.

Lemma 7.4. Let $g \in \mathcal{A}(\mathbb{U})$ and $K_{1}, K_{2} \subset k(\mathbb{U})$ be compact subsets. For all $a \in \mathbb{N}$ there are $D>0$ and $b \in \mathbb{N}$ such that for all $(\gamma, \eta) \in \mathbb{U}^{(2)}$ with $k(\gamma) \in K_{1}, k(\eta) \in K_{2}$

$$
\left|g\left(\gamma^{-1} \eta\right)-g\left(\gamma^{-1}\right)\right| \leq D \frac{(1+\|\eta\|)^{b}}{(1+\|\gamma\|)^{a}} \sum_{j=1}^{n}\|\eta\|^{q_{j}}
$$

Proof. It suffices to show the claim for real-valued $g \in \mathcal{A}(\mathbb{U})$. Let $\gamma^{-1}=$ $(x, v, t)$ and $\eta=\left(\varepsilon_{x}^{-1}(t \cdot v), w, t\right)$. Define the function $G:[0,1] \times \mathbb{U}^{(2)} \rightarrow \mathbb{R}$ by

$$
G(x, v, t, w, h)=g\left((x, v, t) \cdot\left(\varepsilon_{x}^{-1}(t \cdot v), h \cdot w, t\right)\right) .
$$

Hence, we obtain

$$
g\left((x, v, t)\left(\varepsilon_{x}^{-1}(t \cdot v), w, t\right)\right)-g(x, v, t)=\int_{0}^{1} \partial_{h} G(x, v, t, w, s) \mathrm{d} s .
$$

To estimate $\left|\partial_{h} G\right|$ note that $G=g \circ m \circ($ id $\times \delta)$, where $\delta(w, h)=h \cdot w$. Writing $\eta_{h}=\left(\varepsilon_{x}^{-1}(t \cdot v), h \cdot w, t\right)$, one calculates that

$$
\partial_{h} G\left(\gamma^{-1}, \eta, s\right)=\sum_{i, j=1}^{n} \partial_{v_{i}} g\left(\gamma^{-1} \cdot \eta_{h}\right) \cdot \partial_{w_{j}} m_{i}\left(\gamma^{-1}, \eta_{h}\right) \cdot \partial_{h} \delta_{j}(w, s) .
$$

By the structure of $\mathbb{U}$ we can find a compact subset $K_{2} \subset \tilde{K} \subset k(\mathbb{U})$ that is starshaped in the sense that for $(x, v) \in \tilde{K}$ also $(x, h \cdot v) \in \tilde{K}$ holds for all $h \in[0,1]$. Let $T>0$ be such that $g$ vanishes for $t \geq T$. Lemma 6.13 applied to $i\left(K_{1}\right) \cup \tilde{K}$ and $T$ gives $C>0$ and $l \in \mathbb{N}$ with

$$
\left|\partial_{w_{j}} m_{i}\left(\gamma^{-1}, \eta_{h}\right)\right| \leq C\left(1+\left\|\gamma^{-1}\right\|\right)^{l}\left(1+\left\|\eta_{h}\right\|\right)^{l} \leq C(1+\|\gamma\|)^{l^{2}}(1+\|\eta\|)^{l}
$$

for all $\gamma, \eta$ with $k(\gamma) \in K_{1}, k(\eta) \in K_{2}$ and $t<T$. For these $\gamma, \eta$ use the rapid decay condition for $g$ to estimate using Corollary 6.14

$$
\begin{aligned}
\left|\partial_{v_{i}} g\left(\gamma^{-1} \cdot \eta_{h}\right)\right| & \lesssim\left(1+\left\|\gamma^{-1} \cdot \eta_{h}\right\|\right)^{-l^{2}\left(a+l^{2}\right)} \lesssim\left(1+\left\|\eta_{h}^{-1} \cdot \gamma\right\|\right)^{-l\left(a+l^{2}\right)} \\
& \lesssim \frac{\left(1+\left\|\eta_{h}\right\|\right)^{l\left(a+l^{2}\right)}}{(1+\|\gamma\|)^{a+l^{2}}} \leq \frac{(1+\|\eta\|)^{l\left(a+l^{2}\right)}}{(1+\|\gamma\|)^{a+l^{2}}}
\end{aligned}
$$

As $\delta_{j}(w, h)=h^{q_{j}} w_{j}$, it follows that $\left|\partial_{h} \delta_{j}(w, s)\right| \lesssim\left|w_{j}\right| \leq\|\eta\|^{q_{j}}$. Together, these estimates imply the claim.

Lemma 7.5. Let $(M, H)$ be a filtered manifold. Consider the restricted zoom action $\sigma: \mathbb{R}_{>0} \curvearrowright \mathbb{J}$. For $f \in \mathcal{R}$ the operator $\langle\langle f|$ as in Definition 2.1 satisfies $\left\langle\langle f| g \in L^{1}\left(\mathbb{R}_{>0}, \mathbb{J}\right)\right.$ for all $g \in \mathcal{R}$.

Proof. We show that $\left(\lambda \mapsto\left\|\sigma_{\lambda}\left(f^{*}\right) * g\right\|_{I}\right) \in L^{1}\left(\mathbb{R}_{>0}, \frac{\mathrm{d} \lambda}{\lambda}\right)$ holds for all $f, g \in \mathcal{R}$. Because $\sigma_{\lambda}$ for $\lambda>0$ is an isometry with respect to the $I$-norm

$$
\left\|\sigma_{\lambda^{-1}}\left(f^{*}\right) * g\right\|_{I}=\left\|f^{*} * \sigma_{\lambda}(g)\right\|_{I}=\left\|\sigma_{\lambda}\left(g^{*}\right) * f\right\|_{I}
$$

holds for all $f, g \in \mathcal{R}$. Therefore, and as $\mathcal{R}$ is invariant under involution, it will suffice to show

$$
\int_{1}^{\infty}\left\|\sigma_{\lambda}(f) * g\right\|_{I} \frac{\mathrm{d} \lambda}{\lambda}<\infty \quad \text { for all } f, g \in \mathcal{R}
$$

We decompose $f$ as in Lemma 6.10 and write

$$
\sigma_{\lambda}(f) * g=\sum_{j \in\{1, \ldots, m, \infty\}} \sigma_{\lambda}\left(f_{j}\right) * g
$$


with $f_{j} \in \mathcal{R}_{\mathbb{V}_{j}}:=\mathcal{R} \cap \mathcal{A}\left(\mathbb{V}_{j}\right)$ and $f_{\infty} \in \mathrm{C}_{c}^{\infty}\left(\mathbb{V}_{\infty}\right)$. We proceed to decompose this further as in (27). Let $K_{f_{j}}, K_{g}$ be compact subsets for $f_{j}, g$ and let $T>0$ be such that $f_{j}$ and $g$ vanish for $t \geq T$. As before, let $\{\chi, 1-\chi\}$ be a compactly supported partition of unity subordinate to the open cover $V_{j} \times V_{j} \times[0, \infty)$ and $\mathbb{V}_{\infty}$ of $m\left(K_{f_{j}}, K_{g}\right) \times[0, T]$. As noted in Lemma 6.16 we can take the same compact subset $K_{f_{j}} \subset k(\mathbb{U})$ for all $\sigma_{\lambda}\left(f_{j}\right)$. For $\lambda \geq 1$, let $\chi_{\lambda}$ be the scaled version of $\chi$ defined by $\chi_{\lambda}(x, y, t)=\chi\left(x, y, \lambda^{-1} t\right)$. As $\lambda \geq 1,\left\{\chi_{\lambda}, 1-\chi_{\lambda}\right\}$ is still a partition of unity for the above cover of

$$
m\left(K_{f_{j}}, K_{g}\right) \times[0, T]=m\left(K_{\sigma_{\lambda}\left(f_{j}\right)}, K_{g}\right) \times[0, T] .
$$

As before, we have a decomposition

$$
\sigma_{\lambda}\left(f_{j}\right) * g=\left(\sigma_{\lambda}\left(f_{j}\right) * g\right)_{\chi_{\lambda}}+\left(\sigma_{\lambda}\left(f_{j}\right) * g\right)_{\left(1-\chi_{\lambda}\right)} .
$$

Inspecting the argument for the first summand in Theorem 6.15, we can find a $g_{j} \in \mathcal{R}_{\vee_{j}}$ that does not depend on $\lambda$ such that

$$
\left(\sigma_{\lambda}\left(f_{j}\right) * g\right)_{\chi_{\lambda}}=\left(\sigma_{\lambda}\left(f_{j}\right) * g_{j}\right)_{\chi_{\lambda}} .
$$

For the second summand, recall the construction of $\omega \in \mathrm{C}^{\infty}([0, \infty))$ in Theorem 6.15 It follows as in 26 that for $\omega_{\lambda}(t):=\omega\left(\lambda^{-1} t\right)$

$$
\begin{aligned}
\left(\sigma_{\lambda}\left(f_{j}\right) * g\right)_{\left(1-\chi_{\lambda}\right)} & =\left(\sigma_{\lambda}\left(f_{j}\right) \cdot\left(\omega_{\lambda} \circ \theta\right) * g\right)_{\left(1-\chi_{\lambda}\right)} \\
& =\left(\sigma_{\lambda}\left(f_{j} \cdot(\omega \circ \theta)\right) * g\right)_{\left(1-\chi_{\lambda}\right)} .
\end{aligned}
$$

Note that $f_{j} \cdot(\omega \circ \theta) \in \mathrm{C}_{c}^{\infty}\left(\mathbb{V}_{\infty}\right)$. As $\left|\chi_{\lambda}\right|,\left|1-\chi_{\lambda}\right| \leq 1$ for all $\lambda \geq 1$, it suffices to prove (30) for the two cases $f \in \mathrm{C}_{c}^{\infty}\left(\mathbb{V}_{\infty}\right)$ and $g \in \mathcal{R}$, and $f, g \in \mathcal{R}_{\mathbb{U}}$. Here, $\mathcal{R}_{\mathbb{U}}$ denotes all functions $f \in \mathcal{A}(\mathbb{U})$ with $f \circ \phi_{\kappa} \in \mathcal{R}_{\mathbb{V}}$ for an $H$-chart $\kappa: V \rightarrow U$.

Suppose first that $f \in \mathrm{C}_{c}^{\infty}\left(\mathbb{V}_{\infty}\right)$ and $g \in \mathcal{R}$. Let $T, t_{0}>0$ be such that $f(x, y, t)=g(x, y, t)=0$ whenever $t>T$ and $f(x, y, t)=0$ for $t<t_{0}$. For $x \in M$ and $t>0$ we have

$$
\left(\sigma_{\lambda}(f) * g\right)(x, y, t)=\lambda^{d_{H}} t^{-d_{H}} \int_{M} f\left(x, z, \lambda^{-1} t\right) g(z, y, t) \mathrm{d} \nu(z)
$$

which is only non-zero for $t \leq T$. Moreover, it vanishes if $\lambda>T t_{0}^{-1}$ because then $\lambda^{-1} t<t_{0}$ holds for $t \leq T$, so that $f\left(x, z, \lambda^{-1} t\right)=0$. As $\lambda \geq 1$, only $t \geq t_{0}$ have to be considered. As $g$ restricted to $t \geq t_{0}$ is a compactly supported function, we can find a compact subset $K \subset M$ such that $f(x, y, t)=0$ if $(x, y) \notin K \times K$ and $g(x, y, t)=0$ if $t \geq t_{0}$ and $(x, y) \notin K \times K$. Moreover, there is a constant $C>0$ such that $|g| \leq C$ for $t \geq t_{0}$ and $|f| \leq C$. We obtain

$$
\int_{1}^{\infty}\left\|\sigma_{\lambda}(f) * g\right\|_{I} \frac{\mathrm{d} \lambda}{\lambda} \leq T^{2 d_{H}} C^{2} \nu(K)^{2} \int_{1}^{T / t_{0}} \lambda^{d_{H}-1} \mathrm{~d} \lambda<\infty .
$$

Consider now the case that $f, g \in \mathcal{R}_{\mathbb{U}}$. For $\gamma \in \mathbb{U}$ with $r(\gamma)=(x, t)$, write

$$
\begin{aligned}
\left(\sigma_{\lambda}(f) * g^{*}\right)(\gamma) & =\lambda^{d_{H}} \int_{U^{(x, t)}} f\left(x, \lambda \cdot z, \lambda^{-1} t\right) g^{*}\left((x, z, t)^{-1} \gamma\right) \mathrm{d} z \\
& =\int_{U^{(x, t)}} f\left(x, z, \lambda^{-1} t\right) g^{*}\left(\left(x, \lambda^{-1} \cdot z, t\right)^{-1} \gamma\right) \mathrm{d} z
\end{aligned}
$$

Let $K_{f}, K_{g}$ be compact subsets for $f$ and $g$ as in Definition 6.7. As $K_{f}$ is a compact subset for all $\sigma_{\lambda}(f)$, we only need to consider $\gamma \in K:=m\left(K_{f}, K_{g}\right)$ by Theorem 6.15 Moreover, we only need to consider $z \in \mathbb{U}^{(x, t)}$ with $\left(x,\left(\lambda^{-1} t\right) \cdot z\right) \in K_{f}$. Define two functions $R_{1}, R_{2}$ by

$$
\begin{aligned}
R_{1}(\gamma, \eta) & :=g^{*}\left(\eta^{-1} \cdot \gamma\right)-g^{*}(\gamma), \\
R_{2}(x, z, t) & :=f(x, z, t)-f(x, z, 0)
\end{aligned}
$$


We write

$$
\begin{aligned}
\left(\sigma_{\lambda}(f) * g\right)(\gamma)= & g(\gamma) \int f(x, z, 0) \mathrm{d} z+\int f(x, z, 0) R_{1}\left(\gamma,\left(x, \lambda^{-1} \cdot z, t\right)\right) \mathrm{d} z \\
& +\int R_{2}\left(x, z, \lambda^{-1} t\right) g^{*}\left(\left(x, \lambda^{-1} \cdot z, t\right) \cdot \gamma\right) \mathrm{d} z .
\end{aligned}
$$

The first term vanishes as $f \in \mathcal{R}_{U}$. The mean value theorem in Lemma 7.4 applied to $i(K), K_{f}, g$ and $a=d_{H}+1$ yields $D>0$ and $b \in \mathbb{N}$ such that

$$
\begin{aligned}
\left|R_{1}\left(\gamma,\left(x, \lambda^{-1} \cdot z, t\right)\right)\right| & \leq D \frac{\left(1+\left\|\lambda^{-1} \cdot z\right\|\right)^{b}}{(1+\|\gamma\|)^{d_{H}+1}} \sum_{j=1}^{n}\left\|\lambda^{-1} \cdot z\right\|^{w_{i}} \\
& =D \frac{\left(1+\lambda^{-1}\|z\|\right)^{b}}{(1+\|\gamma\|)^{d_{H}+1}} \sum_{j=1}^{n} \lambda^{-w_{i}}\|z\|^{w_{i}} \\
& \leq D \lambda^{-1} \frac{(1+\|z\|)^{b+d_{H}}}{(1+\|\gamma\|)^{d_{H}+1}}
\end{aligned}
$$

for $\gamma \in K$ and $z \in \mathbb{U}^{(x, t)}$ with $\left(x,\left(\lambda^{-1} t\right) \cdot z\right) \in K_{f}$. For the last inequality we used that $\lambda \geq 1$. The usual mean value theorem and the rapid decay of $f$ allow to find $C>0$ such that

$$
\left|R_{2}\left(x, z, \lambda^{-1} t\right)\right| \leq \lambda^{-1} t C(1+\|z\|)^{-\left(l^{2}+1\right)\left(d_{H}+1\right)} .
$$

As $f$ has rapid decay, one can estimate

$$
|f(x, z, 0)| \lesssim(1+\|z\|)^{-b-2 d_{H}-1} .
$$

As $g^{*}$ is rapidly decaying, as well, and using Corollary 6.14 and $\lambda \geq 1$, we find

$$
\begin{aligned}
\left|g^{*}\left(\left(x, \lambda^{-1} z, t\right) \cdot \gamma\right)\right| & \lesssim\left(1+\left\|\left(x, \lambda^{-1} z, t\right) \cdot \gamma\right\|\right)^{-l\left(d_{H}+1\right)} \\
& \lesssim \frac{\left(1+\left\|\left(x, \lambda^{-1} z, t\right)^{-1}\right\|\right)^{l\left(d_{H}+1\right)}}{(1+\|\gamma\|)^{d_{H}+1}} \\
& \lesssim \frac{\left(1+\left\|\left(x, \lambda^{-1} z, t\right)\right\|\right)^{l^{2}\left(d_{H}+1\right)}}{(1+\|\gamma\|)^{d_{H}+1}} \leq \frac{(1+\|z\|)^{l^{2}\left(d_{H}+1\right)}}{(1+\|\gamma\|)^{d_{H}+1}} .
\end{aligned}
$$

Therefore, we obtain for all $(x, t) \in M \times[0, \infty)$

$$
\int\left|\sigma_{\lambda}(f) * g^{*}\right| \mathrm{d} \nu^{(x, t)}=\int\left|\left(\sigma_{\lambda}(f) * g^{*}\right)(x, v, t)\right| \mathrm{d} v \lesssim \lambda^{-1}(1+t) .
$$

As there is a $T>0$ such that $g^{*}$ vanishes for $t \geq T$ this implies $\left\|\sigma_{\lambda}(f) * g^{*}\right\|_{I, r} \lesssim \lambda^{-1}$. For $\|\cdot\|_{I, s}$, replace $\gamma$ by $\gamma^{-1}$ in the estimates above and use Corollary 6.14 to derive similar estimates. The convergence of $\int_{1}^{\infty} \lambda^{-2} \mathrm{~d} \lambda$ finishes the proof of $(30)$.

THEOREM 7.6. For a filtered manifold $(M, H)$ the ${ }^{*}$-subalgebra $\mathcal{R} \subset \rrbracket$ is squareintegrable with respect to the zoom action of $\mathbb{R}_{>0}$.

Denote by $\overline{\mathcal{R}}$ its closure with respect to the $\|\cdot\|_{\mathrm{si}}-$ norm. Then $(\mathbb{J}, \overline{\mathcal{R}})$ is a continuously square-integrable $\mathbb{R}_{>0}-\mathrm{C}^{*}$-algebra.

Proof. The Schwartz type algebra $\mathcal{A}\left(\mathbb{T}_{H} M\right)$ is a ${ }^{*}$-subalgebra of $\mathrm{C}^{*}\left(\mathbb{T}_{H} M\right)$ by Theorem 6.15. Condition (29), which is that the Haar integrals vanish at $t=0$, is preserved when taking the involution or convolution of functions in $\mathcal{R}$. Therefore, $\mathcal{R}$ is a ${ }^{*}$-subalgebra of $\mathbb{J}$. Moreover, it is invariant under the zoom action of $\mathbb{R}_{>0}$, as $\mathcal{A}\left(\mathbb{T}_{H} M\right)$ is invariant by Lemma 6.16 and the $d_{H}$-homogeneity of the Haar system at $t=0$, which follows from 28 . 
To see that $\mathcal{R}$ is dense in $\mathbb{J}$, let $f \in \mathbb{J}$ and $\epsilon>0$. There is a $g \in \mathrm{C}_{c}^{\infty}\left(\mathbb{T}_{H} M\right)$ with $\|f-g\|<\epsilon / 2$. To adjust $g$ to have vanishing integrals at $t=0$, define the function $h \in \mathrm{C}_{c}^{\infty}(M)$ by

$$
h(x)=\int g \mathrm{~d} \nu^{(x, 0)}
$$

for $x \in M$. It satisfies $|h(x)|=\left|\widehat{\pi}_{\text {triv }}\left(q_{x}\left(p_{0}(g)\right)\right)-\widehat{\pi}_{\text {triv }}\left(q_{x}\left(p_{0}(f)\right)\right)\right| \leq\|f-g\|<\varepsilon / 2$ for all $x \in M$. Choose a function $k \in \mathrm{C}_{c}^{\infty}\left(\mathbb{T}_{H} M\right)$ such that $\int k \mathrm{~d} \nu^{(x, 0)}=1$ for all $x \in r\left(p_{0}(\operatorname{supp} g)\right)$ and $\|k\|_{I} \leq 1$. This can be done by defining such a function locally on the charts $\mathbb{V}_{i}$ and pasting them together with a smooth partition of unity. Let $\tilde{g}:=\left(h \circ r_{1}\right) \cdot k$. It is a smooth, compactly supported function on $\mathbb{T}_{H} M$, and $\|\tilde{g}\| \leq \epsilon / 2$. As $g-\tilde{g} \in \mathcal{R}$ and $\|f-(g-\tilde{g})\| \leq \epsilon$, this finishes the proof that $\mathcal{R}$ is dense in $\mathbb{J}$.

Now, the estimate in Lemma 7.5 together with Lemma 2.16 and Remark 2.17 imply that $(\mathbb{J}, \overline{\mathcal{R}})$ is a continuously square-integrable $\mathbb{R}_{>0}$ - $\mathrm{C}^{*}$-algebra.

Hence, the generalized fixed point algebra $\operatorname{Fix}^{\mathbb{R}>0}(\mathbb{J}, \overline{\mathcal{R}})$ is defined as in Definition 2.11. By Lemma 2.13 it is the closed linear span in $\mathcal{M}^{\mathbb{R}>0}(\mathbb{J})$ of

$$
|f\rangle\rangle\left\langle\langle g|=\lim _{i, s} \int_{0}^{\infty} \chi_{i}(\lambda) \sigma_{\lambda}\left(f^{*} * g\right) \frac{\mathrm{d} \lambda}{\lambda} \quad \text { with } f, g \in \mathcal{R}\right.
$$

Here, $\left(\chi_{i}\right)_{i \in I}$ consists of smooth, compactly supported functions $\chi_{i}: \mathbb{R}_{>0} \rightarrow[0,1]$ that converge uniformly on compact subsets to 1 . We can arrange that $\chi_{i}(\lambda)=$ $\chi_{i}\left(\lambda^{-1}\right)$ for all $i \in I$ and $\lambda>0$, which we will assume from now on.

\subsection{The extension of generalized fixed point algebras}

Recall that $\rrbracket_{0}=p_{0}(\mathbb{J})$. The $\mathrm{C}^{*}$-algebra extension for the tangent groupoid in (24) restricts to the short exact sequence of $\mathbb{R}_{>0}-\mathrm{C}^{*}$-algebras

$$
\mathrm{C}_{0}\left(\mathbb{R}_{>0}\right) \otimes \mathbb{K}\left(L^{2} M\right) \longleftrightarrow \mathbb{p _ { 0 }} \longrightarrow \mathbb{J}_{0} .
$$

Proposition 7.7. Let $\mathcal{R}_{0}:=p_{0}(\mathcal{R})$. The zoom action of $\mathbb{R}_{>0}$ on the extension in (31) gives rise to a short exact sequence of generalized fixed point algebras

$$
\mathbb{K}\left(L^{2} M\right) \longleftrightarrow \operatorname{Fix}^{\mathbb{R}>0}(\mathbb{J}, \overline{\mathcal{R}}) \stackrel{\tilde{p}_{0}}{\longrightarrow \operatorname{Fix}^{\mathbb{R}}>0}\left(\mathbb{J}_{0}, \overline{\mathcal{R}_{0}}\right) .
$$

Proof. Proposition 2.24 gives an extension of generalized fixed point algebras

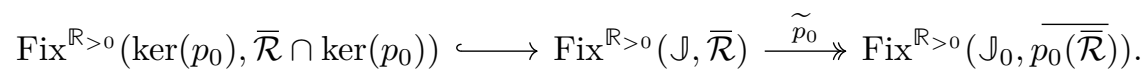

By Remark 2.20 the completion of $p_{0}(\overline{\mathcal{R}})$ with respect to the $\|\cdot\|_{\text {si }}$-norm on the right hand side is $\overline{\mathcal{R}}_{0}$. The isomorphism $p$ from Lemma 6.17 induces an isomorphism

$$
p_{*}: \operatorname{Fix}^{\mathbb{R}_{>0}}\left(\operatorname{ker}\left(p_{0}\right), \overline{\mathcal{R}} \cap \operatorname{ker}\left(p_{0}\right)\right) \rightarrow \operatorname{Fix}^{\mathbb{R}_{>0}}\left(\mathrm{C}_{0}\left(\mathbb{R}_{>0}\right) \otimes \mathbb{K}\left(L^{2} M\right), \tilde{\mathcal{R}}\right) .
$$

where $\tilde{\mathcal{R}}:=p\left(\overline{\mathcal{R}} \cap \operatorname{ker}\left(p_{0}\right)\right)$. By the description of the zoom action on $\mathrm{C}_{0}\left(\mathbb{R}_{>0}\right) \otimes$ $\mathbb{K}\left(L^{2} M\right)$ in Lemma 6.17 it follows that $\tilde{\mathcal{R}}$ is the unique relatively continuous, complete and dense subset by Theorem 2.10 . Lemma 2.29 gives an isomorphism

$$
\Psi: \operatorname{Fix}^{\mathbb{R}>0}\left(\mathrm{C}_{0}\left(\mathbb{R}_{>0}\right) \otimes \mathbb{K}\left(L^{2} M\right), \tilde{\mathcal{R}}\right) \rightarrow \mathbb{K}\left(L^{2} M\right) .
$$

Therefore, we obtain the extension $(32)$. The inclusion of $\mathbb{K}\left(L^{2} M\right)$ into $\operatorname{Fix}^{\mathbb{R}_{>0}}(\mathbb{J}, \overline{\mathcal{R}})$ is given by $\left(\Psi \circ p_{*}\right)^{-1}$.

Proposition 7.8. The generalized fixed point algebra $\mathrm{Fix}^{\mathbb{R}}{ }^{{ }^{0}}\left(\mathbb{\unlhd}_{0}, \overline{\mathcal{R}_{0}}\right)$ is a continuous field of $\mathrm{C}^{*}$-algebras over $M$ with fibre projections

$$
\widetilde{q}_{x}: \operatorname{Fix}^{\mathbb{R}>0}\left(\mathbb{J}_{0}, \overline{\mathcal{R}_{0}}\right) \rightarrow \operatorname{Fix}^{\mathbb{R}>0}\left(J_{x}, \overline{\mathcal{R}_{x}}\right) .
$$


Here $J_{x}:=\operatorname{ker}\left(\widehat{\pi}_{\text {triv }}\right) \triangleleft \mathrm{C}^{*}(G(x))$ and $\mathcal{R}_{x}$ consists of all $f \in \mathcal{S}(G(x))$ with vanishing integral with respect to the Haar measure on $G(x)$.

Proof. This follows from Theorem 2.30

\subsection{A faithful representation on $L^{2}(M)$}

The *-homomorphisms $p_{t}: \mathrm{C}^{*}\left(\mathbb{T}_{H} M\right) \rightarrow \mathbb{K}\left(L^{2} M\right)$ defined in 23 for $t>0$ can be restricted to the ideal $\mathbb{l}$. The restrictions are still surjective. Therefore, they yield strictly continuous representations

$$
\widetilde{p}_{t}: \operatorname{Fix}^{\mathbb{R}>0}(\mathbb{l}, \overline{\mathcal{R}}) \rightarrow \mathcal{M}\left(\mathbb{K}\left(L^{2} M\right)\right)=\mathbb{B}\left(L^{2} M\right) \text { for all } t>0 .
$$

Lemma 7.9. The representation $\widetilde{p}_{1}: \operatorname{Fix}^{\mathbb{R}}>0(\mathbb{l}, \overline{\mathcal{R}}) \rightarrow \mathbb{B}\left(L^{2} M\right)$ is faithful.

Proof. To show that the representation is faithful, observe the following relation between the homomorphisms $p_{t}$ for $t>0$

$$
p_{t} \circ \sigma_{\lambda}=p_{t \lambda^{-1}} \quad \text { for all } t, \lambda>0 .
$$

This equality still holds for the corresponding extensions to the multiplier algebras. As each $T \in \operatorname{Fix}^{\mathbb{R}>0}(\mathbb{J}, \overline{\mathcal{R}})$ is invariant under $\sigma$, it follows that $\widetilde{p}_{t}(T)=\widetilde{p}_{1}(T)$ for all $t>0$. Therefore, $T \in \operatorname{ker}\left(\widetilde{p}_{1}\right)$ implies $\widetilde{p}_{t}(T)=0$ for all $t>0$. For all $f \in \mathbb{I}$ it follows that $p_{t}(T f)=\tilde{p}_{t}(T) p_{t}(f)=0$ for all $t>0$. As $\mathrm{C}^{*}\left(\mathbb{T}_{H} M\right)$ is a continuous field of $\mathrm{C}^{*}$-algebras this implies by continuity that $T f=0$. Since this holds for all $f \in \mathbb{J}$, it follows that $T=0$.

Lemma 7.10. As before, let $\left(\chi_{i}\right)_{i \in I}$ be a net of $\chi_{i} \in \mathrm{C}_{c}^{\infty}\left(\mathbb{R}_{>0}\right)$ that converge uniformly on compact subsets to 1 and satisfy $\chi_{i}\left(\lambda^{-1}\right)=\chi_{i}(\lambda)$ for all $\lambda>0$. Let $f, g \in \mathcal{R}$ and $h=f^{*} * g$. Then the operators $T_{i}(h)$ given by

$$
T_{i}(h) \psi(x)=\int_{0}^{\infty} \chi_{i}(\lambda) \lambda^{-d_{H}} \int h(x, y, \lambda) \psi(y) \mathrm{d} \nu(y) \frac{\mathrm{d} \lambda}{\lambda}
$$

for $\psi \in L^{2}(M), x \in M$, converge strictly to $\widetilde{p}_{1}(|f\rangle\rangle\langle\langle g|)$ as multipliers of $\mathbb{K}\left(L^{2} M\right)$.

Proof. Strict continuity of $\widetilde{p}_{1}$ together with 35 imply that

$$
\tilde{p}_{1}(|f\rangle\rangle\langle\langle g|)=\lim _{i, s} \int_{0}^{\infty} \chi_{i}(\lambda) p_{1}\left(\sigma_{\lambda}\left(f^{*} * g\right)\right) \frac{\mathrm{d} \lambda}{\lambda}=\lim _{i, s} \int_{0}^{\infty} \chi_{i}(\lambda) p_{\lambda}\left(f^{*} * g\right) \frac{\mathrm{d} \lambda}{\lambda} .
$$

The operators $T_{i}(h)$ above are obtained by inserting the definition of $p_{\lambda}$.

Lemma 7.11. Let $p_{*}$ and $\Psi$ be the isomorphisms from (33) and (34). Then following diagram commutes, where the horizontal maps are the inclusions:

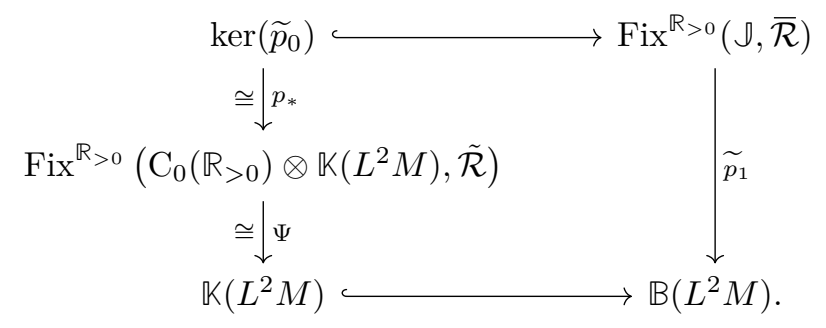


Proof. Let $\psi_{1}, \psi_{2} \in \mathrm{C}_{c}\left(\mathbb{R}_{>0}, \mathbb{K}\left(L^{2} M\right)\right)$. Since $\widetilde{p}_{1}$ is strictly continuous,

$$
\begin{aligned}
\widetilde{p}_{1}\left(\left(p_{*}\right)^{-1}\left(\left|\psi_{1}\right\rangle\right\rangle\left\langle\left\langle\psi_{2}\right|\right)\right) & =\widetilde{p}_{1}\left(\left|p^{-1}\left(\psi_{1}\right)\right\rangle\right\rangle\left\langle\left\langle p^{-1}\left(\psi_{2}\right)\right|\right) \\
& =\widetilde{p}_{1}\left(\lim _{s} \int_{0}^{\infty} \chi_{i}(\lambda) \sigma_{\lambda}\left(p^{-1}\left(\psi_{1}^{*} \psi_{2}\right)\right) \frac{\mathrm{d} \lambda}{\lambda}\right) \\
& =\lim _{s} \int_{0}^{\infty} \chi_{i}(\lambda) p_{\lambda^{-1}}\left(p^{-1}\left(\psi_{1}^{*} \psi_{2}\right)\right) \frac{\mathrm{d} \lambda}{\lambda} \\
& =\int_{0}^{\infty} \psi_{1}\left(\lambda^{-1}\right)^{*} \psi_{2}\left(\lambda^{-1}\right) \frac{\mathrm{d} \lambda}{\lambda}=\Psi\left(\left|\psi_{1}\right\rangle\right\rangle\left\langle\left\langle\psi_{2}\right|\right)
\end{aligned}
$$

holds. As the linear span of $\left.\left|\psi_{1}\right\rangle\right\rangle\left\langle\left\langle\psi_{2}\right|\right.$ with $\psi_{1}, \psi_{2} \in \mathrm{C}_{c}\left(\mathbb{R}_{>0}, \mathbb{K}\left(L^{2} M\right)\right)$ is dense in Fix $\left(\mathrm{C}_{0}\left(\mathbb{R}_{>0}\right) \otimes \mathbb{K}\left(L^{2} M\right), \tilde{\mathcal{R}}\right)$ the claim follows.

Lemma 7.12. Let $h \in \mathcal{R} \cap \operatorname{ker}\left(p_{0}\right)$ and let $T_{i}(h)$ be defined as in (36). Then $\left(T_{i}(h)\right)$ converges in norm in $\mathbb{K}\left(L^{2} M\right)$. In particular, its strict limit as multipliers of $\mathbb{K}\left(L^{2} M\right)$ exists and is contained in $\widetilde{p}_{1}\left(\operatorname{Fix}^{\mathbb{R}>0}(\mathbb{J}, \overline{\mathcal{R}})\right)$.

Proof. As $h \in \mathcal{R} \subset \mathcal{A}\left(\mathbb{T}_{H} M\right)$ vanishes for $t=0$, it can be written as $h=t f$ with $f \in \mathcal{A}\left(\mathbb{T}_{H} M\right)$. By definition of the representation $p_{\lambda}$ in $(23)$, it follows that $p_{\lambda}(h)=\lambda p_{\lambda}(f)$ for all $\lambda>0$. Hence, for all $\lambda>0$

$$
\left\|p_{\lambda}(h)\right\| \leq \lambda\left\|p_{\lambda}(f)\right\| \leq \lambda\|f\| .
$$

We show that $\left(T_{i}(h)\right)$ is Cauchy. Let $T>0$ be such that $h$ vanishes for $t \geq T$. For $j \geq i$, we estimate

$$
\left\|T_{j}(h)-T_{i}(h)\right\| \leq \int_{0}^{\infty}\left(\chi_{j}(\lambda)-\chi_{i}(\lambda)\right)\left\|p_{\lambda}(h)\right\| \frac{\mathrm{d} \lambda}{\lambda} \leq\|f\| \int_{0}^{T}\left(1-\chi_{i}(\lambda)\right) \mathrm{d} \lambda .
$$

As $\chi_{i} \rightarrow 1$ on compact subsets, the claim follows. As $\mathbb{K}\left(L^{2} M\right)$ is complete, it follows that $\left(T_{i}(h)\right)$ converges in norm. The second claim follows as convergence in norm implies strict convergence and $\mathbb{K}\left(L^{2} M\right)$ is contained in $\widetilde{p}_{1}\left(\operatorname{Fix}^{\mathbb{R}>0}(\mathbb{J}, \overline{\mathcal{R}})\right)$ by Lemma 7.11

In conclusion, the generalized fixed point algebra construction for the zoom action on the tangent groupoid of a filtered manifold $(M, H)$ yields an extension

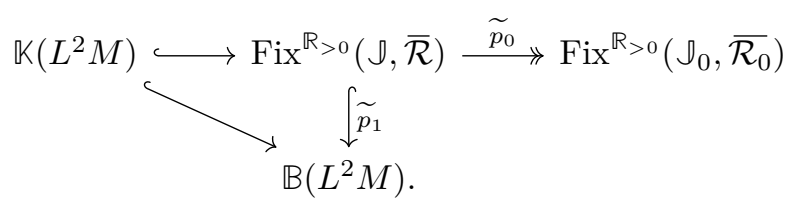

Remark 7.13. In particular, this generalized fixed point algebra construction for the zoom action applies to graded Lie groups. In Ewe20 we showed that it works, in fact, also for homogeneous Lie groups. For these groups the tangent groupoid can be defined as a transformation groupoid as in Example 5.4. The zoom action is defined analogously using the dilations on $G$. 


\section{CHAPTER 8}

\section{Homogeneous distributions and generalized fixed points}

In the classical pseudodifferential calculus on a smooth manifold $M$, the principal symbols of order 0 are smooth functions on the cosphere bundle $S^{*} M$, or, equivalently, smooth 0-homogeneous functions on $T^{*} M \backslash(M \times\{0\})$.

They act on $\mathrm{C}_{0}\left(T^{*} M \backslash(M \times\{0\})\right)$ by multiplication. Under the inverse of the Fourier transform $\mathrm{C}^{*}(T M) \rightarrow \mathrm{C}_{0}\left(T^{*} M\right)$, they act on $\mathbb{J}_{0} \triangleleft \mathrm{C}^{*}(T M)$ by convolution.

We proceed by showing that also in case of a non-trivial filtration, certain homogeneous convolution operators on the bundle of osculating groups $T_{H} M$ are generalized fixed points of the dilatations on $\rrbracket_{0}$.

For an ordinary manifold, it is easy to represent a 0 -homogeneous symbol $F$ on a local chart $V$ as in the generalized fixed point algebra construction. First, one writes

$$
F(x, \xi)=\int_{0}^{\infty} f(x, \lambda \xi) \frac{\mathrm{d} \lambda}{\lambda}
$$

for some smooth, compactly supported function $f$ that vanishes with all derivatives at the zero section. In this case, $f$ can be written as a product $f=f_{1} \cdot f_{2}$ of smooth, compactly supported $f_{1}, f_{2}$ with $f_{1}(x, 0)=f_{2}(x, 0)=0$ for all $x \in V$.

In case of a non-trivial filtration, these factorizations are not so straightforward. We cannot apply the Euclidean Fourier transform as it does not behave well with the group product in the osculating groups. This is why we need a result by Dixmier and Malliavin, which shows that every Schwartz function on a nilpotent Lie group $G$ can be factorized as $f=f_{1} * f_{2}$ with $f_{1}, f_{2} \in \mathcal{S}(G)$. In the following we recall the proof to obtain a bundle version for $T_{H} M$ and to ensure that $f_{1}, f_{2} \in \mathcal{R}_{0}$ if $f$ satisfies certain conditions.

\subsection{Fibred distributions on the bundle of osculating groups}

As a preparation, consider smooth families of distributions on the osculating groups. We will use tempered fibred distributions on $T_{H} M$ (see also vEY19]).

Definition 8.1. For a smooth vector bundle $\pi: E \rightarrow M$, a tempered fibred distribution with compact support in the $M$-direction is a continuous $\mathrm{C}_{c}^{\infty}(M)$-linear map $u: \mathcal{S}(E) \rightarrow \mathrm{C}_{c}^{\infty}(M)$. Denote by $\mathcal{S}_{\mathrm{cp}}^{\prime}(E)$ the linear space of tempered fibred distributions.

For $u \in \mathcal{S}_{\mathrm{cp}}^{\prime}(E)$ and each $x \in M$ there is a tempered distribution $u_{x} \in \mathcal{S}^{\prime}\left(E_{x}\right)$ such that $\langle u, f\rangle(x)=\left\langle u_{x}, f_{x}\right\rangle$ for all $f \in \mathcal{S}(E)$. For $E=T_{H} M$, there is a welldefined convolution

$$
*: \mathcal{S}_{\mathrm{cp}}^{\prime}\left(T_{H} M\right) \times \mathcal{S}\left(T_{H} M\right) \rightarrow \mathcal{S}_{\mathrm{cp}}^{\prime}\left(T_{H} M\right)
$$

which restricts in the fibres to the convolution on the osculating groups. It satisfies analogous properties as in Lemma 3.23. Lemma 3.24 and Lemma 3.25. To define homogeneity of fibred distributions, recall that the dilations yield an $\mathbb{R}_{>0}$-action on $\mathcal{S}\left(T_{H} M\right)$ given by

$$
\left(\sigma_{\lambda} f\right)(x, \xi)=\lambda^{d_{H}} f\left(x, \delta_{\lambda}(\xi)\right) \text { for } \lambda>0, f \in \mathcal{S}\left(T_{H} M\right) \text { and } \xi \in G(x) .
$$


This action can be extended to $\mathcal{S}_{\mathrm{cp}}^{\prime}\left(T_{H} M\right)$ by

$$
\left\langle\sigma_{\lambda *} u, f\right\rangle:=\lambda^{d_{H}}\left\langle u, \sigma_{\lambda^{-1}} f\right\rangle
$$

for $u \in \mathcal{S}_{\mathrm{cp}}^{\prime}\left(T_{H} M\right), f \in \mathcal{S}\left(T_{H} M\right)$ and $\lambda>0$. These allow to extend the notion of kernels and operators of type $\nu$ on homogeneous Lie groups (see for example [FR16, 3.2.9]) to the bundle of osculating groups.

Definition 8.2. Let $G$ be a homogeneous Lie group and let $\nu \in \mathbb{R}$. A tempered distribution $u \in \mathcal{S}^{\prime}(G)$ is called a kernel of type $\nu$ if it is smooth away from zero and $\sigma_{\lambda *}(u)=\lambda^{\nu} u$ for all $\lambda>0$. Denote by $\mathcal{K}^{\nu}(G)$ the space of kernels of type $\nu$.

Remark 8.3. Let $Q$ be the homogeneous dimension of a homogeneous Lie group. The kernels of type $\nu$ are also called $(\nu-Q)$-homogeneous distributions in the literature. This is because they coincide with $(\nu-Q)$-homogeneous functions outside zero.

Definition 8.4. Let $\nu \in \mathbb{R}$. A fibred distribution $u \in \mathcal{S}_{\mathrm{cp}}^{\prime}\left(T_{H} M\right)$ is called a kernel of type $\nu$ if it is smooth away from the zero section and $\sigma_{\lambda *}(u)=\lambda^{\nu} u$ for all $\lambda>0$. Denote by $\mathcal{K}^{\nu}\left(T_{H} M\right)$ the space of kernels of type $\nu$. The corresponding continuous operator $T_{u}: \mathcal{S}\left(T_{H} M\right) \rightarrow \mathcal{S}_{\mathrm{cp}}^{\prime}\left(T_{H} M\right)$ given by $T_{u}(f)=u * f$ is called an operator of type $\nu$.

In particular, $T_{u}$ restricts at $x \in M$ to the right-invariant continuous linear operator $\mathcal{S}(G(x)) \rightarrow \mathcal{S}^{\prime}(G(x))$ given by $f \mapsto u_{x} * f$ for $f \in \mathcal{S}(G(x))$. Moreover, one calculates that an operator $T$ of type $\nu$ satisfies

$$
T\left(\sigma_{\lambda^{-1}} f\right)=\lambda^{\nu} \sigma_{\lambda^{-1}}(T f) \text { for all } \lambda>0 \text { and } f \in \mathcal{S}\left(T_{H} M\right),
$$

see also [FR16, 3.2.7].

Example 8.5. Let $\kappa: V \rightarrow U$ be an $H$-chart with $H$-frame $X$. The differential operators $\langle X\rangle^{\alpha}$ for $\alpha \in \mathbb{N}_{0}^{n}$ can be understood as in Section 3.1 as an element of $\mathcal{S}_{\mathrm{cp}}^{\prime}\left(T_{H} V\right)$ when multiplied by a function $f \in \mathrm{C}_{c}^{\infty}(V)$. It is a kernels of type $-[\alpha]$. The corresponding operator $T_{f \cdot\langle X\rangle^{\alpha}}$ restricts at $x \in M$ to the right-invariant differential operator $f(x) \cdot\langle Y\rangle(x)$ on $G(x)$ defined as in Definition 3.18. To simplify notation, we will write $X_{j}$ for the equivalence class $\left\langle X_{j}\right\rangle \in \Gamma^{\infty}\left(\mathfrak{t}_{H} M\right)$ in the following.

As an analogue under Fourier transform of the functions in $\mathcal{S}_{\mathrm{cp}}\left(T^{*} M\right)$ that vanish with all derivatives at the zero section, define the following subspace of $\mathcal{S}_{\mathrm{cp}}\left(T_{H} M\right)$.

Definition 8.6. For a filtered manifold $(M, H)$, let the space $\mathcal{S}_{0}\left(T_{H} M\right)$ consist of all $f \in \mathcal{S}_{\mathrm{cp}}\left(T_{H} M\right)$ such that for all $H$-charts $\kappa: V \rightarrow U$

$$
\int v^{\alpha}\left(f \circ T \kappa^{-1}\right)(x, v) \mathrm{d} v=0 \quad \text { for all } \alpha \in \mathbb{N}_{0}^{n} \text { and all } x \in U \text {. }
$$

This is well-defined as for a Carnot diffeomorphism $f: U_{1} \rightarrow U_{2}$, the Carnot differential $T f: T_{H} U_{1} \rightarrow T_{H} U_{2}$ preserves polynomials in the fibres.

The Euclidean Fourier transform $\mathcal{F}: \mathcal{S}_{\mathrm{cp}}\left(\mathfrak{t}_{H} M\right) \rightarrow \mathcal{S}_{\mathrm{cp}}\left(\mathfrak{t}_{H}^{*} M\right)$ maps $\mathcal{S}_{0}\left(T_{H} M\right)$ onto the subspace of functions vanishing with all derivatives at the zero section.

Definition 8.7. For a homogeneous Lie group, let $\mathcal{S}_{0}(G)$ consist of all functions $f \in \mathcal{S}(G)$ with $\int_{G} v^{\alpha} f(v) \mathrm{d} v=0$ for all $\alpha \in \mathbb{N}_{0}^{n}$.

The space $\mathcal{S}_{0}(G)$ was defined in [CGGP92 and plays an important role in their calculus. The restrictions give surjective maps $q_{x}: \mathcal{S}_{0}\left(T_{H} M\right) \rightarrow \mathcal{S}_{0}(G(x))$ for all $x \in M$.

Lemma 8.8. The space $\mathcal{S}_{0}\left(T_{H} M\right)$ has the following properties: 
(i) $\mathcal{S}_{0}\left(T_{H} M\right)$ is a ${ }^{*}$-subalgebra of $\mathcal{S}_{\mathrm{cp}}\left(T_{H} M\right)$,

(ii) $\mathcal{S}_{0}\left(T_{H} M\right)$ is closed in $\mathcal{S}_{\mathrm{cp}}\left(T_{H} M\right)$ with respect to the Schwartz semi-norms,

(iii) $u * f \in \mathcal{S}_{0}\left(T_{H} M\right)$ for all $u \in \mathcal{K}^{\nu}\left(T_{H} M\right), \nu \in \mathbb{R}$ and $f \in \mathcal{S}_{0}\left(T_{H} M\right)$,

(iv) $X * f \in \mathcal{S}_{0}\left(T_{H} M\right)$ for all smooth, compactly supported sections $X: M \rightarrow$ $\mathfrak{t}_{H} M$ and $f \in \mathcal{S}_{0}\left(T_{H} M\right)$.

Proof. It is clear that $\mathcal{S}_{0}\left(T_{H} M\right)$ is a linear subspace of $\mathcal{S}_{\mathrm{cp}}\left(T_{H} M\right)$. It is left to show for (i) that $\mathcal{S}_{0}\left(T_{H} M\right)$ is closed under involution and convolution. In $H$-coordinates and for $v \in G(x)$

$$
\left(v^{-1}\right)^{\alpha}=(-v)^{\alpha}=(-1)^{|\alpha|} v^{\alpha} .
$$

Moreover, by the polynomial group law in Proposition 3.17 one can write $v \cdot w$ for $x, w \in G(x)$ as a finite sum of $c_{\beta, \gamma}(x) v^{\beta} w^{\gamma}$ with smooth functions $c_{\beta, \gamma}$.

We show (ii) on an $H$-chart $V$. Pick a homogeneous quasi-norm on $T_{H} V$ as in (16) and let the Schwartz seminorms be defined analogously to Definition 3.20. For $f \in \mathcal{S}_{\mathrm{cp}}\left(T_{H} V\right)$ and $\alpha \in \mathbb{N}_{0}^{n}$, we estimate

$$
\left|\int v^{\alpha} f(x, v) \mathrm{d} v\right| \leq\|f\|_{[\alpha]+d_{H}-1} \int(1+\|v\|)^{-d_{H}+1} \mathrm{~d} v \quad \text { for all } x \in V .
$$

The integral converges by Lemma 3.14. It follows that for a sequence $\left(f_{k}\right)_{k \in \mathbb{N}}$ in $\mathcal{S}_{0}\left(T_{H} V\right)$ which converges in $\mathcal{S}_{\mathrm{cp}}\left(T_{H} V\right)$, the limit lies in $\mathcal{S}_{0}\left(T_{H} V\right)$ as well.

The property (iii) is the bundle version of Gel83 Lemma 4]. For (iv) let $X \in \Gamma_{c}^{\infty}\left(\mathfrak{t}_{H} V\right)$ for an $H$-chart $V$. Then one can write $X=\sum c_{j} X_{j}$ with $c_{j} \in \mathrm{C}_{c}^{\infty}(V)$ with respect to the $H$-frame $\left\{X_{1}, \ldots, X_{n}\right\}$. The $c_{j} X_{j}$ define kernels of type $-q_{j}$ by Example 8.5. Hence, (iii) implies the claim.

\subsection{The Theorem of Dixmier and Malliavin}

The theorem was proved in DM78, see also Cas16. The following is the foundation for the factorization theorem.

THEOREM 8.9 ([DM78, 2.5]). Let $\left(\beta_{k}\right)$ be a sequence of positive real numbers. Then there is a sequence $\left(\alpha_{k}\right)$ with $\alpha_{k} \leq \beta_{k}$ for all $k \geq 1$ and a function $h \in \mathcal{S}(\mathbb{R})$ such that

$$
\sum_{k=0}^{p}(-1)^{k} \alpha_{k} \delta^{(2 k)} * h \rightarrow \delta \quad \text { in } \mathcal{S}^{\prime}(\mathbb{R}) \text { as } p \rightarrow \infty .
$$

In the following we show a bundle version of the Dixmier and Malliavin Theorem. Note that we do not obtain a factorization with two functions but as a finite sum of convolutions, as we do a construction in local coordinates. However, this is good enough for the generalized fixed point algebra construction.

THEOREM 8.10 ([DM78, 7.2]). Let $(M, H)$ be a filtered manifold. For each $f \in \mathcal{S}_{\mathrm{cp}}\left(T_{H} M\right)$ the following holds:

(i) There are finitely many $g_{i}, h_{i} \in \mathcal{S}_{\mathrm{cp}}\left(T_{H} M\right)$ such that $f=\sum g_{i} * h_{i}$.

(ii) If $f \in \mathcal{S}_{0}\left(T_{H} M\right)$, one can find a factorization as in (i) with $g_{i} \in \mathcal{S}_{0}\left(T_{H} M\right)$.

Proof. Consider an open cover of $M$ by $H$-charts $\kappa_{i}: V_{i} \rightarrow U_{i}$. As $f$ has compact support in the $M$-direction, there are $\rho_{i} \in \mathrm{C}_{c}^{\infty}\left(V_{i}\right)$ for $i=1, \ldots, m$ such that

$$
f(x, v)=\sum_{i=1}^{m} \rho_{i}(x) f(x, v) .
$$

Therefore, it will suffice to show the claim for $f \in \mathcal{S}_{\mathrm{cp}}\left(T_{H} V\right)$ for an $H$-chart $\kappa: V \rightarrow$ $U$. Let $\left(X_{1}, \ldots, X_{n}\right)$ denote the $H$-frame on $V$. For $x \in V$, the equivalence classes 
of $\left\{X_{1}(x), \ldots, X_{n}(x)\right\}$ form a strong Malcev basis for all osculating groups $G(x)$ with $x \in V$. Therefore, by [CG90 1.2.8]

$$
\begin{aligned}
\Phi: V \times \mathbb{R}^{n} & \rightarrow T_{H} V \\
\left(x, t_{1}, \ldots, t_{n}\right) & \mapsto\left(x, \exp \left(t_{n}\left\langle X_{n}\right\rangle(x)\right) \cdots \exp \left(t_{1}\left\langle X_{1}\right\rangle(x)\right)\right)
\end{aligned}
$$

defines a diffeomorphism. Moreover, by CG90 1.2.11] it transports the Lebesgue measure on $\mathbb{R}^{n}$ to the Haar measure induced by the corresponding exponential coordinates in each fibre.

Denote by $Y_{i}$ for $j=1, \ldots, n$ the family of right-invariant differential operators on the osculating groups corresponding to $X_{j}$. For $k, m \in \mathbb{N}_{0}, \alpha \in \mathbb{N}_{0}^{n}$ let

$$
M_{k, m, \alpha}:=\sup _{(x, v) \in T_{H} V}\left|v^{m} Y^{\alpha}\left(Y_{1}^{2 k} f\right)(x, v)\right|,
$$

which are finite as $f$ belongs to the Schwartz space. Choose a sequence of positive real numbers $\left(\beta_{k}\right)$ such that

$$
\sum_{k=0}^{\infty} \beta_{k} M_{k, m, \alpha}<\infty \quad \text { for all } m \in \mathbb{N}_{0}^{n}, \alpha \in \mathbb{N}_{0}^{n},
$$

see also Cas16 4.4]. Now by Theorem 8.9, there is a sequence $\alpha_{k} \leq \beta_{k}$ for $k \geq 1$ and a function $h_{1} \in \mathcal{S}(\mathbb{R})$ with

$$
\sum_{k=0}^{p}(-1)^{k} \alpha_{k} \delta^{(2 k)} * h_{1} \rightarrow \delta \quad \text { in } \mathcal{S}^{\prime}(\mathbb{R}) \text { as } p \rightarrow \infty .
$$

Consider the map $\Phi_{1}: V \times \mathbb{R} \rightarrow T_{H} V$ given by $\left(x, t_{1}\right) \mapsto\left(x, \exp \left(t_{1} X_{1}\right)\right)$. It is a polynomial homomorphism in the fibres. Note that $\left(\Phi_{1}(x)\right)_{*}\left(\delta^{(k)}\right)=X_{1}(x)^{k} \in$ $\mathcal{S}^{\prime}(G(x))$ for all $x \in V$. By Lemma 3.25 it follows that for a function $\omega \in \mathrm{C}_{c}^{\infty}(V)$ which is constant 1 on $r(\operatorname{supp} f)$

$$
\sum_{k=0}^{p}(-1)^{k} \alpha_{k} X_{1}^{2 k} *\left(\Phi_{1}\right)_{*}\left(\omega \otimes h_{1}\right) \rightarrow \omega \otimes \delta \quad \text { in } \mathcal{S}_{\mathrm{cp}}^{\prime}\left(T_{H} M\right) \text { as } p \rightarrow \infty .
$$

holds. It follows that

$$
f *\left(\sum_{k=0}^{p}(-1)^{k} \alpha_{k} X_{1}^{2 k} *\left(\Phi_{1}\right)_{*}\left(\omega \otimes h_{1}\right)\right) \rightarrow f \quad \text { in } \mathcal{S}_{\mathrm{cp}}\left(T_{H} M\right) \text { as } p \rightarrow \infty .
$$

Note that for each $p \in \mathbb{N}$ by Lemma 3.24

$$
f *\left(\sum_{k=0}^{p}(-1)^{k} \alpha_{k} X_{1}^{2 k} *\left(\Phi_{1}\right)_{*}\left(\omega \otimes h_{1}\right)\right)=\left(\sum_{k=0}^{p}(-1)^{k} \alpha_{k} Y_{1}^{2 k} f\right) *\left(\Phi_{1}\right)_{*}\left(\omega \otimes h_{1}\right) .
$$

By the choice of $\beta_{k}$ in $(39)$ and the definition of $M_{k, m, \alpha}$ in (38), the sequence $\sum_{k=0}^{p}(-1)^{k} \alpha_{k} Y_{1}^{2 k} f$ is Cauchy with respect to all semi-norms. Therefore, it converges to a function $g_{1} \in \mathcal{S}_{\mathrm{cp}}\left(T_{H} V\right)$. It follows that $f=g_{1} *\left(\Phi_{1}\right)_{*}\left(\omega \otimes h_{1}\right)$.

Now, the same procedure is applied to $g_{1}$ and $\Phi_{2}\left(x, t_{2}\right):=\left(x, \exp \left(t_{2} X_{2}\right)\right)$. Subsequently, we obtain

$$
f=\left(\ldots\left(g_{n} *\left(\Phi_{n}\right)_{*}\left(\omega \otimes h_{n}\right)\right) * \ldots\right) *\left(\Phi_{1}\right)_{*}\left(\omega \otimes h_{1}\right)
$$

for a function $g_{n} \in \mathcal{S}_{\mathrm{cp}}\left(T_{H} V\right)$ and $h_{1}, \ldots, h_{n} \in \mathcal{S}(\mathbb{R})$. Define $h \in \mathcal{S}_{\mathrm{cp}}\left(V \times \mathbb{R}^{n}\right)$ by $h(x, t):=\omega(x) h_{1}\left(t_{1}\right) \cdots h_{n}\left(t_{n}\right)$. One computes that

$$
g_{n} *\left(\Phi_{n}\right)_{*}\left(h_{n}\right) * \cdots *\left(\Phi_{1}\right)_{*}\left(h_{1}\right)(x, v)=\int_{\mathbb{R}^{n}} h(x, t) g_{n}\left((x, v) \Phi(x, t)^{-1}\right) \mathrm{d} t .
$$

As the Lebesgue measures on $\mathbb{R}^{n}$ is transferred to the Haar system by $\Phi$, it follows that $f=g_{n} *\left(\Phi^{*} h\right)$. This finishes the proof of (i) 
If $f \in \mathcal{S}_{0}\left(T_{H} V\right)$, note that $g_{1} \in \mathcal{S}_{0}\left(T_{H} V\right)$ by (iv) and (ii) of Lemma 8.8 Inductively, it follows that $g_{n} \in \mathcal{S}_{0}\left(T_{H} V\right)$.

\subsection{Operators of type 0 as generalized fixed points}

In this section, we show that $\operatorname{Fix}^{\mathbb{R}>0}\left(\mathbb{J}_{0}, \overline{\mathcal{R}_{0}}\right)$ is isomorphic to the $\mathrm{C}^{*}$-algebra generated by the operators $T_{u}$ for kernels $u \in \mathcal{S}_{\mathrm{cp}}^{\prime}\left(T_{H} M\right)$ of type 0 .

Proposition 8.11 ([FS82, 1.65]). Let $f \in \mathcal{R}_{0}$. Then

$$
\int_{0}^{\infty} \chi_{i}(\lambda) \sigma_{\lambda}(f) \frac{\mathrm{d} \lambda}{\lambda}
$$

converges in $\mathcal{S}_{\mathrm{cp}}^{\prime}\left(T_{H} M\right)$ to a kernel of type 0.

We will show that, conversely, every $u \in \mathcal{K}^{0}\left(T_{H} M\right)$ can be written as an average over the dilation action as above. First, this is proved for $u=\omega \otimes \delta$, where $\delta$ denotes the delta distribution in the fibres and $\omega \in \mathrm{C}_{c}^{\infty}(M)$, which is a kernel of type 0 .

Lemma 8.12. For every $\omega \in \mathrm{C}_{c}^{\infty}(M)$ there are finitely many $f_{j} \in \mathcal{R}_{0}$ and $g_{j} \in$ $\mathcal{S}_{0}\left(T_{H} M\right), j=1, \ldots, l$, such that

$$
\sum_{j=1}^{l} \int_{0}^{\infty} \chi_{i}(\lambda) \sigma_{\lambda}\left(f_{j}^{*} * g_{j}\right) \frac{\mathrm{d} \lambda}{\lambda} \rightarrow \omega \otimes \delta \quad \text { in } \mathcal{S}_{\mathrm{cp}}^{\prime}\left(T_{H} M\right) .
$$

Proof. It suffices to show this for $\omega \in \mathrm{C}_{c}^{\infty}(V)$ for an $H$-chart $V$ of $M$. As noted in [CGGP92], one can find a $\phi \in \mathcal{S}_{0}\left(T_{H} V\right)$ with

$$
\omega \otimes \delta=\lim _{i} \int_{\mathbb{R}_{>0}} \chi_{i}(\lambda) \sigma_{\lambda}(\phi)(x) \frac{\mathrm{d} \lambda}{\lambda} .
$$

For example, take a function $h \in \mathrm{C}_{c}^{\infty}\left(\mathbb{R}_{>0}\right)$ with $\int_{0}^{\infty} h\left(\lambda^{-1}\right) \frac{\mathrm{d} \lambda}{\lambda}=1$. Define $\psi \in$ $\mathcal{S}\left(\mathfrak{t}_{H}^{*} V\right)$ with $\psi(x, \xi)=\omega(x) h(\|\xi\|)$, where $\|\cdot\|$ is a homogeneous quasi-norm on $\mathfrak{t}_{H}^{*} V$ which is smooth outside the zero section. The invariance of the Haar measure on $\mathbb{R}_{>0}$ implies that $\int_{0}^{\infty} \psi\left(x, \alpha_{\lambda^{-1}}(\xi)\right) \frac{\mathrm{d} \lambda}{\lambda}=\omega(x)$ for all $x \in V$ and $\xi \neq 0$. Therefore, $\phi \in \mathcal{S}_{0}\left(T_{H} V\right)$ can be taken as the inverse Euclidean Fourier transform of $\psi$.

Now, $\phi$ needs to be factorized appropriately. By the proof of the Dixmier Malliavin Theorem in 8.10 one can find $k \in \mathcal{S}_{0}\left(T_{H} V\right)$ and $l \in \mathcal{S}\left(T_{H} V\right)$ such that $\phi=k * l$. Following [FS82, 1.60] and using the $H$-frame $\left\{X_{1}, \ldots, X_{n}\right\}$ on $V, k$ can be written as $k=\sum_{j=1}^{n} X_{j} k_{j}$ with $k_{1}, \ldots, k_{n} \in \mathcal{S}_{0}\left(T_{H} V\right)$. Therefore, using (9)

$$
\phi=\sum_{j=1}^{n}\left(X_{j} k_{j}\right) * l=\sum_{j=1}^{n} k_{j} *\left(Y_{j} l\right)
$$

holds. Using Proposition 3.19 one obtains that $\int\left(Y_{j} l\right)(x, v) \mathrm{d} v=0$ for all $x \in V$ holds. Consequently, the claim holds with $f_{j}:=k_{j}^{*} \in \mathcal{S}_{0}\left(T_{H} V\right)$ and $g_{j}:=Y_{j} g \in \mathcal{R}_{0}$ for $j=1, \ldots, n$.

Corollary 8.13. Every $u \in \mathcal{K}^{0}\left(T_{H} M\right)$ can be written as a finite sum of

$$
\lim _{i} \int_{0}^{\infty} \chi_{i}(\lambda) \sigma_{\lambda}\left(f^{*} * g\right) \frac{\mathrm{d} \lambda}{\lambda}
$$

with $f \in \mathcal{S}_{0}\left(T_{H} M\right)$ and $g \in \mathcal{R}_{0}$.

Proof. Choose $\omega \in \mathrm{C}_{c}^{\infty}(M)$ with $\left.\chi\right|_{r(\operatorname{supp} u)} \equiv 1$. Let $f_{j} \in \mathcal{S}_{0}\left(T_{H} M\right)$ and $g_{j} \in \mathcal{R}_{0}$ for $j=1, \ldots, l$ be functions as in Lemma 8.12. Then use the homogeneity 
of $\kappa$ to compute for $h \in \mathcal{S}_{\mathrm{cp}}\left(T_{H} M\right)$

$$
\begin{aligned}
u * h & =u *(\omega \otimes \delta * h)=\lim \left(\sum_{j=1}^{l} \int_{0}^{\infty} \chi_{i}(\lambda) u *\left(\sigma_{\lambda}\left(f_{j} * g_{j}\right) * h\right) \frac{\mathrm{d} \lambda}{\lambda}\right) \\
& =\lim \left(\sum_{j=1}^{l} \int_{0}^{\infty} \chi_{i}(\lambda) \sigma_{\lambda}\left(\left(u * f_{j}\right) * g_{j}\right) * h \frac{\mathrm{d} r}{r}\right) \\
& =\sum_{j=1}^{l}\left(\lim \int_{0}^{\infty} \chi_{i}(\lambda) \sigma_{\lambda}\left(\left(u * f_{j}\right) * g_{j}\right) \frac{\mathrm{d} \lambda}{\lambda}\right) * h
\end{aligned}
$$

Here we used that $u *(f * g)=(u * f) * g$ for all $\phi, \psi \in \mathcal{S}_{\mathrm{cp}}\left(T_{H} M\right)$. The last equality holds as $u * f_{j}$ lies in $\mathcal{S}_{0}\left(T_{H} M\right)$ for all $j=1, \ldots, n$ by Lemma 8.8 , so that the expression in the bottom line converges in $\mathcal{S}_{\mathrm{cp}}^{\prime}\left(T_{H} M\right)$ by Proposition 8.11

Example 8.14. Suppose $G$ is a homogeneous Lie group of homogeneous dimension $Q$ equipped with a smooth homogeneous quasi-norm. Let $k \in \mathrm{C}^{\infty}(G)$ be a $(-Q)$ homogeneous function with vanishing mean value, that is,

$$
\int_{G} k(x) u(\|x\|) \mathrm{d} x=0
$$

for all $u \in L^{1}\left(\mathbb{R}_{>0}, \frac{\mathrm{d} \lambda}{\lambda}\right)$. By $[\mathbf{F S 8 2}, 6.13,6.19]$ its principal value distribution $\mathrm{PV}(k) \in \mathcal{S}^{\prime}(G)$ is defined by

$$
\langle\mathrm{PV}(k), \psi\rangle=\lim _{\varepsilon \rightarrow 0} \int_{\|x\|>\varepsilon} k(x) \psi(x) \mathrm{d} x \quad \text { for } \psi \in \mathcal{S}(G) .
$$

Let $\omega \in \mathrm{C}_{c}^{\infty}\left(\mathbb{R}_{>0}\right)$ be a function with $\int_{\mathbb{R}_{>0}} \lambda^{Q} \omega(\lambda) \frac{\mathrm{d} \lambda}{\lambda}=1$. Then we can define a function $g \in \mathcal{S}(G)$ by $g(x)=\omega(\|x\|) k(x /\|x\|)$ for $x \in G$. Its integral vanishes by the vanishing mean value condition for $k$ :

$$
\int_{G} \omega(\|x\|) k(x /\|x\|) \mathrm{d} x=\int_{G} \omega(\|x\|)\|x\|^{Q} k(x) \mathrm{d} x=0 .
$$

Moreover, for $x \neq 0$,

$$
\int_{0}^{\infty} \sigma_{\lambda}(g)(x) \frac{\mathrm{d} \lambda}{\lambda}=\int_{0}^{\infty} \lambda^{Q} \omega(\lambda\|x\|) k(x /\|x\|) \frac{\mathrm{d} \lambda}{\lambda}=\|x\|^{-Q} k(x /\|x\|)=k(x)
$$

holds. By $[\mathbf{F S 8 2}, 6.13]$ this implies that PV $(k)=\lim _{i} \int_{0}^{\infty} \chi_{i}(\lambda) \sigma_{\lambda}(g) \frac{\mathrm{d} \lambda}{\lambda}+c \cdot \delta$ for some $c \in \mathbb{C}$. Writing $\delta$ as in Lemma 8.12 one obtains a representation of $\mathrm{PV}(k)$ as an average over the dilations.

Remark 8.15. Let $\nu>0$ and $f \in \mathcal{S}_{0}\left(T_{H} M\right)$. Then

$$
\int_{0}^{\infty} \chi_{i}(\lambda) \lambda^{-\nu} \sigma_{\lambda}(f) \frac{\mathrm{d} \lambda}{\lambda}
$$

converges to a smooth $\left(\nu-d_{H}\right)$-homogeneous function outside the zero section. By Lemma 3.14 it is locally integrable at the zero section. It follows that it defines a tempered fibred distribution. It is in fact a kernel of type $\nu$. Conversely, every kernel of type $\nu>0$ can be written in this form by a similar argument as in the proof of Corollary 8.13 .

Remark 8.16. Suppose $L$ is a right-invariant $\nu$-homogeneous differential operator on a homogeneous Lie group $G$, such that $L$ and $L^{t}$ are hypoelliptic. Then by Fol75, 2.1] and Gel83, Thm. 3], $L$ admits a fundamental solution $\kappa \in \mathcal{S}^{\prime}(G)$, 
that is $L * \kappa=\delta$. Let $Q$ denote the homogeneous dimension of $G$. If $\nu<Q, \kappa$ is a kernel of type $\nu$ and is uniquely determined. If $\nu \geq Q, \kappa$ is of the form

$$
\kappa=\kappa_{0}+p(x) \ln (\|x\|)
$$

where $\kappa_{0}$ is a kernel of type $\nu, p$ a polynomial which is homogeneous of degree $\nu-Q$ and $\|\cdot\|$ denotes a homogeneous quasi-norm on $G$. Convolution operators with kernels of this form are still homogeneous when their domain is restricted to $\mathcal{S}_{0}(G)$.

Because of this structure of the fundamental solutions, pseudodifferential operators of order $m$ in the calculus of Christ, Geller, Głowacki and Polin CGGP92 are defined to have a homogeneous expansion of their convolution kernel of the form $\sum_{j=-m}^{\infty} \kappa_{j}$ with $\kappa_{j}$ kernels of type $j$ for $j<Q$ and $\kappa_{j}$ of the form in 40 for $j \geq Q$. In the calculus of van Erp and Yuncken this is reflected by considering essentially homogeneous distributions and not only homogeneous distributions (see vEY19, Example 26]).

The description of the kernels of type 0 above is already very close to the generalized fixed point algebra construction. We compare now the corresponding convolution operators to elements of $\operatorname{Fix}^{\mathbb{R}>0}\left(\mathbb{J}_{0}, \overline{\mathcal{R}}\right) \subset \mathcal{M}^{\mathbb{R}>0}\left(\mathbb{J}_{0}\right)$. The latter is a continuous field of $\mathrm{C}^{*}$-algebras with fibres $\operatorname{Fix}^{\mathbb{R}>0}\left(J_{x}, \overline{\mathcal{R}_{x}}\right)$ by Proposition 7.8 To understand these fixed point algebras better, note that the restrictions of the left regular representations $\lambda_{x}: \mathrm{C}^{*}(G(x)) \rightarrow \mathbb{B}\left(L^{2} G(x)\right)$ to $J_{x}$ are still non-degenerate for all $x \in M$.

Lemma 8.17. For each graded Lie group $G$, the restriction of the left regular representation $\lambda: \mathrm{C}^{*}(G) \rightarrow \mathbb{B}\left(L^{2} G\right)$ to $J=\operatorname{ker}\left(\widehat{\pi}_{\text {triv }}\right)$ is a non-degenerate representation.

Proof. Suppose $\psi \in L^{2}(G)$ is such that $f * \psi=0$ holds for all $f \in J$. As $\mathrm{C}^{*}(G)$ acts by right-invariant operators on $L^{2}(G)$, this is equivalent to $\widehat{f}(\pi) \widehat{\psi}(\pi)=0$ for all $f \in J$ and for almost all $\pi \in \widehat{G}$ by the Plancherel Theorem, see $(13)$. The ideal $J \triangleleft \mathrm{C}^{*}(G)$ is liminal. Hence for $\pi \in \widehat{J}=\widehat{G} \backslash\left\{\pi_{\text {triv }}\right\}$ we have that $\widehat{f}(\pi) \widehat{\psi}(\pi)=0$ for all $f \in J$ is equivalent to $\mathbb{K}\left(\mathcal{H}_{\pi}\right) \widehat{\psi}(\pi)=0$. But as $\widehat{\psi}(\pi)$ is Hilbert-Schmidt, this means that $\widehat{\psi}(\pi)=0$ for $\pi \neq \pi_{\text {triv }}$. The Plancherel measure is supported within the representations corresponding to orbits of maximal dimension sequence. In particular, $\left\{\pi_{\text {triv }}\right\}$ has measure zero and, therefore, $\psi=0$ must hold.

Consequently, as the restriction is also faithful, the multiplier algebras $\mathcal{M}\left(J_{x}\right)$ can be identified with the idealizer of $\lambda\left(J_{x}\right)$ in $\mathbb{B}\left(L^{2} G(x)\right)$. Therefore, elements of the generalized fixed point algebra $\operatorname{Fix}^{\mathbb{R}_{>0}}\left(J_{x}, \overline{\mathcal{R}_{x}}\right)$ can be viewed as bounded, rightinvariant operators on $L^{2}(G(x))$. This allows us to reprove the following theorem of KS71.

THEOREM 8.18. Every operator $T$ of type 0 extends uniquely to a family $T_{x}$ of bounded operators $L^{2}(G(x)) \rightarrow L^{2}(G(x))$.

Proof. Note that if such an extension exists, it is unique as $\mathcal{S}(G(x)) \subset$ $L^{2}(G(x))$ is dense for each $x \in M$. Let $f, g \in \mathcal{R}_{0}$ and consider the kernel of type 0 given by

$$
u=\lim _{i} \int_{0}^{\infty} \chi_{i}(\lambda) \sigma_{\lambda}\left(f^{*} * g\right) \frac{\mathrm{d} \lambda}{\lambda} .
$$

If we can show that $T_{u, x}: \psi \mapsto u_{x} * \psi$ defined on $\mathcal{S}(G(x))$ extends to an operator in $\mathbb{B}\left(L^{2} G(x)\right)$, the claim follows as every kernel of type 0 is a finite sum of kernels of this form by Corollary 8.13. Let $h \in \mathcal{R}_{x}$ and $\psi \in \mathcal{S}(G(x))$. The description of $\left.\left|q_{x}(f)\right\rangle\right\rangle\left\langle\left\langle q_{x}(g)\right|\right.$ as a strict limit as in Lemma 2.13 shows that

$$
\widetilde{\lambda}_{x}\left(\left|q_{x}(f)\right\rangle\right\rangle\left\langle\left\langle q_{x}(g)\right|\right)\left(\lambda_{x}(h) \psi\right)=T_{u, x}\left(\lambda_{x}(h) \psi\right) .
$$


As the restricted left regular representation is non-degenerate by Lemma 8.17 . $\left\{\lambda_{x}(h) \psi \mid h \in \mathcal{R}_{x}\right.$ and $\left.\psi \in \mathcal{S}(G(x))\right\}$ is dense in $L^{2}(G(x))$. Hence, the operator $\widetilde{\lambda}_{x}\left(\left|q_{x}(f)\right\rangle\right\rangle\left\langle\left\langle q_{x}(g)\right|\right)$ is the unique continuous extension of $T_{u, x}$.

Proposition 8.19. The generalized fixed point algebra $\operatorname{Fix}^{\mathbb{R}>0}\left(\mathbb{J}_{0}, \overline{\mathcal{R}_{0}}\right)$ is the $\mathrm{C}^{*}$ closure of the operators of type 0 .

Proof. This follows from the one-to-one correspondence between the operators of type 0 and the linear span of $|f\rangle\rangle\left\langle\langle g|\right.$ with $f, g \in \mathcal{R}_{0}$ obtained from Proposition 8.11, Corollary 8.13 and Theorem 8.18. The latter is dense in the $\mathrm{C}^{*}$-algebra $\operatorname{Fix}^{\mathbb{R}>0}\left(\rrbracket_{0}, \overline{\mathcal{R}_{0}}\right)$.

Corollary 8.20. Let $G$ be a homogeneous Lie group, $J_{G}=\operatorname{ker}\left(\widehat{\pi}_{\text {triv }}\right)$ and let $\mathcal{R}_{G}$ consist of all $f \in \mathcal{S}(G)$ with $\int_{G} f(v) \mathrm{d} v=0$. The generalized fixed point algebra $\operatorname{Fix}^{\mathbb{R}_{>0}}\left(J_{G}, \overline{\mathcal{R}_{G}}\right)$ is the $\mathrm{C}^{*}$-completion of the operators of type 0 on $G$. 


\section{CHAPTER 9}

\section{Comparison to calculi on filtered manifold}

In Chapter 7, we obtained for a filtered manifold $(M, H)$ and the zoom action an extension of generalized fixed point algebras

$$
\mathbb{K}\left(L^{2} M\right) \longleftrightarrow \operatorname{Fix}^{\mathbb{R}>0}(\mathbb{J}, \overline{\mathcal{R}}) \stackrel{\widetilde{p}_{0}}{\longrightarrow} \operatorname{Fix}^{\mathbb{R}>0}\left(\mathbb{J}_{0}, \overline{\mathcal{R}_{0}}\right) .
$$

In this chapter, it is proved that it coincides with the $\mathrm{C}^{*}$-completion of certain order zero pseudodifferential extensions defined in the literature. Namely, we will compare it to the calculus by van Erp and Yuncken in vEY19 for filtered manifolds and the calculus of Fischer, Ruzhansky and Fermanian-Kammerer in [FR16 FFK17 for graded Lie groups.

Let us mention that there are also other calculi related to filtered manifolds, for example, the calculus of Melin for filtered manifolds in the unpublished manuscript Mel82, the calculus of Beals and Greiner for Heisenberg manifolds in BG88, the calculus of Taylor for contact manifolds in [Tay84], or the calculus of Christ, Geller, Głowacki and Polin in [CGGP92 for homogeneous Lie groups.

\subsection{Van Erp and Yuncken's calculus}

The pseudodifferential calculus for filtered manifolds developed by van Erp and Yuncken is also based on the tangent groupoid and the zoom action. Note that they use a version of the tangent groupoid which is a field over $\mathbb{R}$ and not $[0, \infty)$. In this section, we outline of their construction and compare it to the generalized fixed point algebra approach.

Recall the theory of fibred distributions on Lie groupoids and their convolutions as in $\mathbf{v E Y 1 9}$, Section 2], see also [LMV17.

Definition 9.1 ( $\mathbf{v E Y 1 9} 6])$. Let $\mathcal{G}$ be a Lie groupoid with unit space $\mathcal{G}^{(0)}$ and range and source $r, s: \mathcal{G} \rightarrow \mathcal{G}^{(0)}$. An $r$-fibred distribution, respectively an $s$-fibred distribution, is a continuous $\mathrm{C}^{\infty}\left(\mathcal{G}^{(0)}\right)$-linear map $u: \mathrm{C}^{\infty}(\mathcal{G}) \rightarrow \mathrm{C}^{\infty}\left(\mathcal{G}^{(0)}\right)$, where the $\mathrm{C}^{\infty}\left(\mathcal{G}^{(0)}\right)$-module structure on $\mathrm{C}^{\infty}(\mathcal{G})$ is induced by the range, respectively the source map. The spaces of $r$ - and $s$-fibred distributions are denoted by $\mathcal{E}_{r}^{\prime}(\mathcal{G})$ and $\mathcal{E}_{s}^{\prime}(\mathcal{G})$

There are well-defined convolution products $*: \mathcal{E}_{\pi}^{\prime}(\mathcal{G}) \times \mathcal{E}_{\pi}^{\prime}(\mathcal{G}) \rightarrow \mathcal{E}_{\pi}^{\prime}(\mathcal{G})$ for $\pi=r, s$ that turn $\mathcal{E}_{\pi}^{\prime}(\mathcal{G})$ into an associative algebra [LMV17, 20].

Definition 9.2. A subset $X \subset \mathcal{G}$ is proper if the restricted range and source maps

$$
\left.r\right|_{X}: X \rightarrow \mathcal{G}^{(0)} \quad \text { and }\left.\quad s\right|_{X}: X \rightarrow \mathcal{G}^{(0)}
$$

are proper. For $\pi=r, s$, let $\Omega_{\pi}$ be the bundle of smooth densities tangent to the range fibres, respectively source fibres, of $\mathcal{G}$. Let $\mathrm{C}_{\mathrm{p}}^{\infty}\left(\mathcal{G} ; \Omega_{\pi}\right)$ denote the space of smooth sections $f$ such that $\operatorname{supp}(f)$ is proper.

Then $\mathrm{C}_{\mathrm{p}}^{\infty}\left(\mathcal{G} ; \Omega_{r}\right)$ is a right ideal in $\mathcal{E}_{r}^{\prime}(\mathcal{G})$, whereas $\mathrm{C}_{\mathrm{p}}^{\infty}\left(\mathcal{G} ; \Omega_{s}\right)$ is a left ideal in $\mathcal{E}_{s}^{\prime}(\mathcal{G})$ (see [vEY19, 9] and [LMV17, 21]).

The zoom action from Definition 5.8 induces an $\mathbb{R}_{>0}$-action $\alpha_{*}$ on $\mathcal{E}_{r}^{\prime}\left(\mathbb{T}_{H} M\right)$ by automorphisms. Each $\alpha_{\lambda *}$ restricted to $\mathrm{C}_{c}^{\infty}\left(\mathbb{T}_{H} M ; \Omega_{r}\right)$ coincides with $\sigma_{\lambda^{-1}}$ as 
in Lemma 6.16 when identifying $\mathrm{C}_{c}^{\infty}\left(\mathbb{T}_{H} M ; \Omega_{r}\right)$ with $\mathrm{C}_{c}^{\infty}\left(\mathbb{T}_{H} M\right)$ using the left invariant Haar system.

Definition 9.3 ( $\mathbf{v E Y 1 9}, 18,19])$. A properly supported $\mathbb{P} \in \mathcal{E}_{r}^{\prime}\left(\mathbb{T}_{H} M\right)$ is essentially homogeneous of weight $m \in \mathbb{R}$ if

$$
\alpha_{\lambda *}(\mathbb{P})-\lambda^{m} \mathbb{P} \in \mathrm{C}_{\mathrm{p}}^{\infty}\left(\mathbb{T}_{H} M ; \Omega_{r}\right) \text { for all } \lambda>0 .
$$

The space of these distributions is denoted by $\Downarrow_{H}^{m}(M)$.

A distribution $P \in \mathcal{E}_{r}^{\prime}(M \times M)$ is an $H$-pseudodifferential kernel of order $\leq m$ if there is a $\mathbb{P} \in \Downarrow_{H}^{m}(M)$ that restricts to $P$ at $t=1$. Denote by $\Psi_{H}^{m}(M)$ the space of these kernels. For $P \in \Psi_{H}^{m}(M)$, the operator

$$
\mathrm{Op}(P): \mathrm{C}^{\infty}(M) \rightarrow \mathrm{C}^{\infty}(M), \quad \mathrm{Op}(P) f(x)=\int_{M} P(x, y) f(y)
$$

is the corresponding $H$-pseudodifferential operator.

Moreover, they define the principal cosymbol of $P \in \Psi_{H}^{m}(M)$ by extending it to a $\mathbb{P} \in \Psi_{H}^{m}(M)$ and restricting $\mathbb{P}$ to $t=0$. To make this independent of the choice of the extension, the space of cosymbols is defined as follows.

Definition 9.4 ( $\mathbf{v E Y 1 9} 34,35])$. For $m \in \mathbb{R}$, let $\operatorname{Ess}_{H}^{m}(M)$ consist of all properly supported $u \in \mathcal{E}_{r}^{\prime}\left(T_{H} M\right)$ such that

$$
\delta_{\lambda *}(u)-\lambda^{m} u \in \mathrm{C}_{\mathrm{p}}^{\infty}\left(T_{H} M ; \Omega_{r}\right) \quad \text { for all } \lambda>0 .
$$

Here, $\delta_{*}$ is induced by the dilations in the fibres of $T_{H} M$. Define

$$
\Sigma_{H}^{m}(M):=\operatorname{Ess}_{H}^{m}(M) / \mathrm{C}_{\mathrm{p}}^{\infty}\left(T_{H} M ; \Omega_{r}\right) .
$$

The principal cosymbol of $P \in \Psi_{H}^{m}(M)$ is defined by extending $P$ to $\mathbb{P} \in \Psi_{H}^{m}(M)$ and setting

$$
s_{H}^{m}(P):=\left[\mathbb{P}_{0}\right] \in \Sigma_{H}^{m}(M) .
$$

Van Erp and Yuncken show in $\mathbf{v E Y 1 9}$ 47] that the wave front set of $\mathbb{P} \in$ $\mathbb{w}_{H}^{m}(M)$ is contained in the conormal to $M \times[0, \infty)$. This implies by vEY19, 48] that $\mathbb{P}$ belongs, in fact, to the space of proper $r$-fibred distributions $\mathcal{E}_{r, s}^{\prime}\left(\mathbb{T}_{H} M\right)$ as defined in $\mathbf{v E Y 1 9}, 11]$. As $\mathrm{C}_{\mathrm{p}}^{\infty}\left(\mathbb{T}_{H} M ; \Omega_{r}\right)$ is a two-sided ideal in $\mathcal{E}_{r, s}^{\prime}\left(\mathbb{T}_{H} M\right)$, it is then easy to see that $\bigcup_{m \in \mathbb{Z}} \Psi_{H}^{m}(M)$ is a $\mathbb{Z}$-graded algebra, see $\left.\mathbf{v E Y 1 9}, 49\right]$. Moreover, there is a well-defined involution on $\mathcal{E}_{r, s}^{\prime}\left(\mathbb{T}_{H} M\right)$ by LMV17, 20]. We summarize now the main properties of the calculus.

Proposition 9.5 (wEY19, 36, 37, 38, 49, 50, 52, 53], [DH17, 3.6]). The pseudodifferential calculus on a filtered manifold $(M, H)$ satisfies:

(i) For $m \in \mathbb{Z}$ there is a short exact sequence

$$
\Psi_{H}^{m-1}(M) \longleftrightarrow \Psi_{H}^{m}(M) \stackrel{s_{H}^{m}}{\longrightarrow} \Sigma_{H}^{m}(M) .
$$

The inclusion is well-defined, which can be seen by considering the map $\Downarrow_{H}^{m-1}(M) \rightarrow \Psi_{H}^{m}(M)$ with $\mathbb{P} \mapsto t \mathbb{P}$ for $\mathbb{P} \in \Psi_{H}^{m-1}(M)$, which does not change the kernel at $t=1$.

(ii) For $P \in \Psi_{H}^{m}(M)$ and $Q \in \Psi_{H}^{l}(M)$ with $m, l \in \mathbb{R}$, the convolution is in $\Psi_{H}^{m+l}(M)$ and $s_{H}^{m+l}(P * Q)=s_{H}^{m}(P) * s_{H}^{l}(Q)$.

(iii) For $P \in \Psi_{H}^{m}(M)$ and $m \in \mathbb{R}$, the formal adjoint $P^{*}$ is in $\Psi_{H}^{m}(M)$ and $s_{H}^{m}\left(P^{*}\right)=s_{H}^{m}(P)^{*}$.

(iv) For $k \geq 0$ and $r$ the step of the filtration, the following regularities hold

$$
\begin{array}{r}
\Psi_{H}^{-d_{H}-k r-1}(M) \subset \mathrm{C}_{\mathrm{p}}^{k}\left(M \times M ; \Omega_{r}\right), \\
\bigcap_{m \in \mathbb{Z}} \Psi_{H}^{m}(M)=\mathrm{C}_{\mathrm{p}}^{\infty}\left(M \times M ; \Omega_{r}\right) .
\end{array}
$$


Remark 9.6. Dave and Haller extend in DH17 the pseudodifferential calculus to operators acting between sections of vector bundles $E, F$ over $M$. In this case, distributions in $\mathcal{E}_{r}^{\prime}\left(\mathbb{T}_{H} M ; \operatorname{hom}\left(s^{*}(\mathbb{E}), r^{*}(\mathbb{F})\right) \otimes \Omega_{r}\right)$ are used, where $\mathbb{E}=E \times \mathbb{R}$ and $\mathbb{F}=F \times \mathbb{R}$ are the vector bundles over the unit space $M \times \mathbb{R}$.

Frequently, global exponential coordinates as in [vEY17, 15] are used. They identify an open subset $\widetilde{\mathbb{W}}$ of $\mathfrak{t}_{H} M \times \mathbb{R}$, the Lie algebroid of $\mathbb{T}_{H} M$, with an open, zoom invariant neighbourhood $\mathbb{W}$ of

$$
T_{H} M \times 0 \cup \Delta_{M} \times(0, \infty) \subset \mathbb{T}_{H} M .
$$

Here, $\Delta_{M}$ denotes the diagonal in $M \times M$.

Suppose $\varphi_{1} \in \mathrm{C}^{\infty}(M \times M)$ is constant 1 on $\Delta_{M}$ and vanishes outside $\mathbb{W}_{1}$. Then $\varphi \in \mathrm{C}^{\infty}\left(\mathbb{T}_{H} M\right)$ defined by $\varphi:=\varphi_{1} \circ\left(r_{1}, s_{1}\right)$ is called an exponential cutoff in $\mathbf{v E Y 1 9}$ 27]. It is invariant under the zoom action. It is shown in [vEY19, 27] that each $\mathbb{P} \in \rrbracket_{H}^{m}(M)$ differs from a $\mathbb{Q} \in \Downarrow_{H}^{m}(M)$ supported on $\mathbb{W}$ by an element of $\mathrm{C}_{p}^{\infty}\left(\mathbb{T}_{H} M ; \Omega_{r}\right)$, namely, set $\mathbb{Q}=\varphi \mathbb{P}$ for an exponential cutoff $\varphi$.

As we used the Schwartz type algebra in the construction of the generalized fixed point algebra $\operatorname{Fix}^{\mathbb{R}_{>0}}(\mathbb{J}, \overline{\mathcal{R}})$, the corresponding operators on $L^{2}(M)$ as in Lemma 7.10 have compactly supported kernels. Therefore, we compare it to the following variant of van Erp and Yuncken's calculus with compact instead of proper supports.

Definition 9.7. Let $\Downarrow_{H, c}^{m}(M)$ consist of all $\mathbb{P} \in \Downarrow_{H}^{m}(M)$ such that there is a compact subset $K \subset M \times M$ such that all $\mathbb{P}_{t}$ for $t \neq 0$ are supported in $K$. Likewise, $\operatorname{Ess}_{H, c}^{m}(M)$ denotes all $u \in \operatorname{Ess}_{H}^{m}(M)$ with compact support in the $x$-direction and

$$
\Sigma_{H, c}^{m}(M):=\operatorname{Ess}_{H, c}^{m}(M) / \mathrm{C}_{c}^{\infty}\left(T_{H} M ; \Omega_{r}\right) .
$$

Lemma 9.8. Let $m \in \mathbb{Z}$. The pseudodifferential extension of order $m$ in (41) restricts to a short exact sequence

$$
\Psi_{H, c}^{m-1}(M) \longleftrightarrow \Psi_{H, c}^{m}(M) \stackrel{s_{H}^{m}}{\longrightarrow} \Sigma_{H, c}^{m}(M)
$$

Proof. This can be shown analogously to [vEY19, 36, 37, 38]. For surjectivity of the principal cosymbol map, extend a compactly supported $u \in \operatorname{Ess}_{H, c}^{m}(M)$ as in $\mathbf{v E Y 1 9}$ 36] to the constant distribution $\tilde{u} \in \mathcal{E}_{r}^{\prime}\left(\mathfrak{t}_{H} M \times \mathbb{R}\right)$ given by $\tilde{\mathbb{u}}_{t}=u$. Using an exponential cutoff $\varphi$ for a $\varphi_{1} \in \mathrm{C}_{c}^{\infty}(M \times M)$ that is constant 1 on a neighbourhood of $\Delta_{r(\operatorname{supp} u)} \subset M \times M$ and zero outside of $\mathbb{W}_{1}$, one obtains an $u \in \Downarrow_{H, c}^{m}(M)$ that extends $u$.

We will first compare the space of principal cosymbols of order 0 to the generalized fixed point algebra at $t=0$, namely, $\operatorname{Fix}^{\mathbb{R}>0}\left(\mathbb{J}_{0}, \overline{\mathcal{R}_{0}}\right)$. Consider the following bundle version of the space of approximately homogeneous distributions defined in [Tay84, 2].

Definition 9.9. A tempered fibred distribution $u \in \mathcal{S}_{\mathrm{cp}}^{\prime}\left(T_{H} M\right)$ is approximately 0 -homogeneous if

(i) $u$ is smooth outside the zero section,

(ii) $u=u_{1}+u_{2}$ for some $u_{1} \in \mathcal{S}_{\mathrm{cp}}\left(T_{H} M\right)$ and $u_{2} \in \mathcal{E}_{\mathrm{cp}}^{\prime}\left(T_{H} M\right)$,

(iii) $\sigma_{\lambda}(u)-u \in \mathcal{S}_{\mathrm{cp}}\left(T_{H} M\right)$ for all $\lambda>0$.

Denote by $\mathfrak{H}_{\mathrm{cp}}^{0}\left(T_{H} M\right)$ the space of approximately 0-homogeneous distributions.

By Tay84, 2.2, 2.4] they are closely related to kernels of type 0, defined in Definition 8.4

Proposition 9.10. There is a surjective linear map $\Phi: \mathfrak{H}_{\mathrm{cp}}^{0}\left(T_{H} M\right) \rightarrow \mathcal{K}^{0}\left(T_{H} M\right)$ with $\operatorname{ker}(\Phi)=\mathfrak{H}_{\mathrm{cp}}^{0}\left(T_{H} M\right) \cap \mathrm{C}_{\mathrm{cp}}^{\infty}\left(T_{H} M\right)$. 
Proof. By the bundle version of [Tay84, 2.4] every $u \in \mathfrak{H}_{\mathrm{cp}}^{0}\left(T_{H} M\right)$ can be written as $u=f+w$ with $f \in \mathrm{C}_{\mathrm{cp}}^{\infty}\left(T_{H} M\right)$ and $w \in \mathcal{K}^{0}\left(T_{H} M\right)$. In fact, it is shown in Tay84, 2.2], that $\widehat{u} \in \mathrm{C}^{\infty}\left(\mathfrak{t}_{H}^{*} M\right)$ admits a radial limit $\tilde{w}(x, \xi):=$ $\lim _{\lambda \rightarrow \infty} u(x, \lambda \xi)$, which defines a smooth, 0 -homogeneous function $\tilde{w}$. Therefore, $\tilde{w}$ extends to a tempered fibred distribution and $w$ is its inverse under Fourier transform. The above decomposition of $u$ is unique as kernels of type 0 coincide with smooth $\left(-d_{H}\right)$-homogeneous functions outside the zero section. Therefore, $\mathrm{C}_{\mathrm{cp}}^{\infty}\left(T_{H} M\right) \cap \mathcal{K}^{0}\left(T_{H} M\right)=\{0\}$ and $u \mapsto w$ is a well-defined linear map.

For surjectivity, let $w \in \mathcal{K}^{0}\left(T_{H} M\right)$ be a kernel of type 0. The Euclidean Fourier transform $\widehat{w} \in \mathcal{S}^{\prime}\left(\mathfrak{t}_{H}^{*} M\right)$ is smooth and 0-homogeneous outside the zero section $M \times\{0\}$. Take a smooth cutoff function $\chi \in \mathrm{C}^{\infty}\left(\mathfrak{t}_{H}^{*} M\right)$ which vanishes near the zero section and is constant 1 outside an $r$-compact set. Then $u=\mathcal{F}^{-1}(\chi \widehat{w})$ is an approximately 0-homogeneous distribution by [Tay84, 2.2] and one can write

$$
u=w-\mathcal{F}^{-1}((1-\chi) \widehat{w}) .
$$

The latter is smooth as $(1-\chi) \widehat{w} \in \mathcal{E}_{\mathrm{cp}}^{\prime}\left(\mathfrak{t}_{H} M\right)$, so that $\Phi(u)=w$. The claim concerning the kernel follows from the uniqueness of the decomposition above and the definition of $\Phi$.

Note that $\mathfrak{H}_{\mathrm{cp}}^{0}\left(T_{H} M\right)$ is larger than the space $\operatorname{Ess}_{H, c}^{0}(M)$ we consider. However, the following result holds. See [DH17, 3.8] for a more general result for $\Sigma_{H}^{m}(M)$.

Lemma 9.11. There is a linear bijection $\Theta: \Sigma_{H, c}^{0}(M) \rightarrow \mathcal{K}^{0}\left(T_{H} M\right)$.

Proof. The map $\Phi$ in Proposition 9.10 induces a bijective, linear map

$$
\mathfrak{H}_{\mathrm{cp}}^{0}\left(T_{H} M\right) / \operatorname{ker}(\Phi) \rightarrow \mathcal{K}^{0}\left(T_{H} M\right) .
$$

We show that there is a linear bijection $\Sigma_{H, c}^{0}(M) \rightarrow \mathfrak{H}_{\mathrm{cp}}^{0}\left(T_{H} M\right) / \operatorname{ker}(\Phi)$. The inclusion $\iota$ : $\operatorname{Ess}_{H, c}^{0}(M) \hookrightarrow \mathfrak{H}_{\mathrm{cp}}^{0}\left(T_{H} M\right)$ induces a linear map to the quotient

$$
\psi: \operatorname{Ess}_{H, c}^{0}(M) \rightarrow \mathfrak{H}_{\mathrm{cp}}^{0}\left(T_{H} M\right) / \operatorname{ker}(\Phi) .
$$

To see that it is surjective, let $u \in \mathfrak{H}_{\mathrm{cp}}^{0}\left(T_{H} M\right)$ and choose a function $\omega \in \mathrm{C}_{c}^{\infty}\left(T_{H} M\right)$ which is constant 1 in a neighbourhood of $r(\operatorname{supp} u) \times\{0\}$ and consider $\omega u \in$ $\mathcal{E}_{\text {cp }}^{\prime}\left(T_{H} M\right)$. It is essentially 0-homogeneous as for all $\lambda>0$

$$
\sigma_{\lambda}(\omega u)-\omega u=-\sigma_{\lambda}((1-\omega) u)+\sigma_{\lambda}(u)-u+(1-\omega) u
$$

is smooth. It is also compactly supported as the left hand side is. Moreover, $u-\omega u=(1-\omega) u$ is smooth, so that $\psi(\omega u)=[u]$.

Finally, we show that $\operatorname{ker}(\psi)=\mathrm{C}_{c}^{\infty}\left(T_{H} M\right)$. If $u \in \operatorname{Ess}_{H, c}^{0}(M)$ is contained in the kernel of $\psi$, it is smooth by the description of the kernel of $\Phi$. It is also compactly supported. So it must lie in $\mathrm{C}_{c}^{\infty}\left(T_{H} M\right)$. As $\mathrm{C}_{c}^{\infty}\left(T_{H} M\right) \subset \mathrm{C}_{\mathrm{cp}}^{\infty}\left(T_{H} M\right)$ the converse inclusion holds, too.

By the description of $\mathcal{K}^{0}\left(T_{H} M\right)$ inside a generalized fixed point algebra in Proposition 8.19, we obtain a linear map

$$
\Theta: \Sigma_{H, c}^{0}(M) \rightarrow \operatorname{Fix}^{\mathbb{R}>0}\left(\rrbracket_{0}, \overline{\mathcal{R}}\right)
$$

with dense image. However, it is not clear from the proof that it is a *-homomorphism for the convolution and involution of distributions. This will be shown later.

It was observed in DS14 Theorem 3.7] that classical pseudodifferential operators on a manifold $M$ can be written as averages over the zoom action of functions $f \in \mathcal{A}(\mathbb{T} M)$ with $f_{0} \in \mathcal{S}_{0}(T M)$. We will show this for filtered manifolds for the order zero case.

Lemma 9.12. Let $g \in \mathrm{C}_{c}^{\infty}\left(\mathfrak{t}_{H}^{*} M \times \mathbb{R}\right)$ vanish at all points $(x, 0,0)$ for $x \in M$. Then $\int_{0}^{\infty} \chi_{i}(\lambda) g(x, \lambda \cdot \xi, \lambda) \frac{\mathrm{d} \lambda}{\lambda}$ converges in $\mathcal{S}_{\mathrm{cp}}^{\prime}\left(\mathfrak{t}_{H}^{*} M\right)$. 
Proof. We show that for each $(x, \xi) \in \mathfrak{t}_{H}^{*} M$

$$
K(x, \xi):=\lim _{i} \int_{0}^{\infty} \chi_{i}(\lambda) g(x, \lambda \cdot \xi, \lambda) \frac{\mathrm{d} \lambda}{\lambda}
$$

converges and satisfies for all $\alpha \in \mathbb{N}_{0}^{n}$

$$
\sup _{(x, \xi)}\left|\partial_{x}^{\alpha} K(x, \xi)\right|<\infty .
$$

As $g$ vanishes at all points $(x, 0,0)$, it can be written as

$$
g(x, \xi, t)=\sum_{j=1}^{n} \xi_{j} g_{j}(x, \xi, t)+t g_{t}(x, \xi, t)
$$

with $g_{j}, g_{t} \in \mathrm{C}_{c}^{\infty}\left(\mathfrak{t}_{H}^{*} M \times \mathbb{R}\right)$ for $j=1, \ldots, n$. Since they are compactly supported, there are $R, T>0$ such that $g_{j}(x, \xi, t)=g_{t}(x, \xi, t)=0$ for $j=1, \ldots, n$ whenever $\|\xi\|>R$ or $t>T$. It follows that $|K(x, \xi)| \leq T(R+1) \max _{j=1, \ldots, n, t}\left\|g_{j}\right\|_{\infty}$. This shows the claim for $\alpha=0$. Derivatives in the $x$-direction give an expression of the same form so it holds for all $\alpha \in \mathbb{N}_{0}^{n}$.

Proposition 9.13. Let $\Theta$ be the map from Lemma 9.11. For $\mathbb{P} \in \Downarrow_{H, c}^{0}(M)$ there is $f \in \mathcal{R}$ with $f_{0} \in \mathcal{S}_{0}\left(T_{H} M\right)$ such that

$$
\mathbb{P}_{1}-\int_{0}^{\infty} p_{1}\left(\sigma_{\lambda}(f)\right) \frac{\mathrm{d} \lambda}{\lambda} \in \mathrm{C}_{c}^{\infty}(M \times M) \quad \text { and } \quad \Theta\left(\left[\mathbb{P}_{0}\right]\right)=\int_{0}^{\infty} \sigma_{\lambda}\left(f_{0}\right) \frac{\mathrm{d} \lambda}{\lambda} .
$$

Proof. By vEY19, Lemma 27 and Lemma 42] $\mathbb{P}$ differs by a kernel in $\mathrm{C}_{\mathrm{p}}^{\infty}\left(\mathbb{T}_{H} M\right)$ from an element $\mathbb{Q} \in \Downarrow_{H}^{0}(M)$ which is supported in the global exponential coordinate patch and is homogeneous on the nose outside $[-1,1]$. Using that $\mathbb{P}$ has a uniform compact support $K \subset M \times M$ for $t \neq 0$, one can arrange that the same holds for the kernel in $\mathrm{C}_{\mathrm{p}}^{\infty}\left(\mathbb{T}_{H} M\right)$ and that $\mathbb{Q} \in \Psi_{H, c}^{0}(M)$. So assume, without loss of generality, that $\mathbb{P}$ has is supported in the global exponential coordinate patch and is homogeneous on the nose outside $[-1,1]$.

Let $\tilde{\mathbb{P}} \in \mathcal{E}_{r}^{\prime}\left(\mathfrak{t}_{H} M \times \mathbb{R}\right)$ be the pullback under the exponential map and $\widehat{\mathbb{P}}=$ $\mathcal{F}(\tilde{\mathbb{P}}) \in \mathrm{C}^{\infty}\left(\mathfrak{t}_{H}^{*} M \times \mathbb{R}\right)$. Here, $\mathcal{F}$ is the fibrewise Euclidean Fourier transform $\mathcal{F}: \mathcal{S}_{r}^{\prime}\left(\mathfrak{t}_{H} M \times \mathbb{R}\right) \rightarrow \mathcal{S}_{r}^{\prime}\left(\mathfrak{t}_{H}^{*} M \times \mathbb{R}\right)$. As remarked in vEY19, 7.3], $\widehat{\mathbb{P}}$ is approximately homogeneous for the $\mathbb{R}_{>0}$-action $\beta_{\lambda}(x, \xi, t)=(x, \lambda \cdot \xi, \lambda t)$ on $\mathfrak{t}_{H}^{*} M \times \mathbb{R}$. This is a dilation action when considering $\mathfrak{t}_{H}^{*} M \times \mathbb{R} \stackrel{\pi}{\rightarrow} M$ as a graded vector bundle over $M$ with the original dilations on $\mathfrak{t}_{H}^{*} M$ and the usual scaling on $\mathbb{R}$ as observed in vEY19, 7.3].

Now we use [vEY19 Prop. 43], which is based on the bundle version of Tay84, 2.2]. It allows to find an $A \in \mathrm{C}_{\mathrm{cp}}^{\infty}\left(\mathfrak{t}_{H}^{*} M \times \mathbb{R} \backslash M \times 0 \times 0\right)$ homogeneous of degree 0 such that $\widehat{\mathbb{P}}-\chi A \in \mathcal{S}_{\mathrm{cp}, \pi}\left(\mathfrak{t}_{H}^{*} M \times \mathbb{R}\right)$ for any smooth cutoff function $\chi$ that vanishes in a neighbourhood of the zero section $M \times 0 \times 0$ and is constant 1 outside a $\pi$-compact set. Now choose $g \in \mathrm{C}_{c}^{\infty}\left(\mathfrak{t}_{H}^{*} M \times \mathbb{R}\right)$ vanishing with all derivatives at all points $(x, 0,0)$ such that for all $(\xi, t) \neq(0,0)$

$$
A(x, \xi, t)=\int_{0}^{\infty} g(x, \lambda \xi, \lambda t) \frac{\mathrm{d} \lambda}{\lambda}
$$

Then $\int_{0}^{\infty} g(x, \lambda \xi, \lambda t) \frac{\mathrm{d} \lambda}{\lambda}$ converges in $\mathcal{S}_{\mathrm{cp}}^{\prime}\left(\mathfrak{t}_{H}^{*} M\right)$ for $t=0$ by Proposition 8.11 and for $t>0$ by Lemma 9.12 . We write for all $t \geq 0$ and a cutoff function $\chi$ as above

$$
\widehat{\mathbb{P}}_{t}-\int_{0}^{\infty} g(x, \lambda \xi, \lambda t) \frac{\mathrm{d} \lambda}{\lambda}=\left(\widehat{\mathbb{P}}_{t}-\chi(\xi, t) A_{t}\right)-(1-\chi(\xi, t)) \int_{0}^{\infty} g(x, \lambda \xi, \lambda t) \frac{\mathrm{d} \lambda}{\lambda} .
$$


The first part lies in $\mathcal{S}_{\mathrm{cp}}\left(\mathfrak{t}_{H}^{*} M\right)$ and the second in $\mathcal{E}_{r}^{\prime}\left(\mathfrak{t}_{H}^{*} M\right)$ with compact support in the $x$-direction. So that we obtain under inverse Fourier transform

$$
\tilde{\mathbb{P}}_{t}-\int_{0}^{\infty}\left(\tilde{\sigma}_{\lambda} \circ \mathcal{F}^{-1}(g)\right)(t) \frac{\mathrm{d} \lambda}{\lambda} \in \mathrm{C}_{\mathrm{cp}}^{\infty}\left(\mathfrak{t}_{H} M\right) .
$$

Here, $\tilde{\sigma}$ denotes the zoom action on $\mathfrak{t}_{H} M \times \mathbb{R}$.

As $\mathbb{P}$ has uniform compact support, there is an exponential cutoff $\varphi$ built from a compactly supported $\varphi_{1} \in \mathrm{C}_{c}^{\infty}(M \times M)$ such that $\varphi \mathbb{P}=\mathbb{P}$. Let $f:=\varphi \mathcal{F}^{-1}(g)$, which is an element of $\mathcal{R}$ with $f_{0} \in \mathcal{S}_{0}\left(T_{H} M\right)$. We obtain for all $t \geq 0$, using that $\varphi$ is invariant under the zoom action,

$$
\mathbb{P}_{t}-\int_{0}^{\infty} p_{t}\left(\sigma_{\lambda}(f)\right) \frac{\mathrm{d} \lambda}{\lambda}=\varphi_{t}\left(\mathbb{P}_{t}-\int_{0}^{\infty} p_{t} \circ \sigma_{\lambda} \circ \mathcal{F}^{-1}(g) \frac{\mathrm{d} \lambda}{\lambda}\right) .
$$

For $t>0$, this is contained in $\mathrm{C}_{c}^{\infty}(M \times M)$. For $t=0$, note that $\varphi_{0}=1$ on the support of $\mathbb{P}_{0}$ and $g$ in the $x$-direction. As $\mathbb{P}_{0} \in \Sigma_{H, c}^{0}(M) \subseteq \mathfrak{H}_{\text {cp }}^{0}\left(T_{H} M\right)$, the claim follows from Proposition 9.10

Lemma 9.14. For $f \in \mathcal{R}$ with $f_{0} \in \mathcal{S}_{0}\left(T_{H} M\right)$

$$
\int_{0}^{\infty} \chi_{i}(\lambda) \sigma_{\lambda}(f) \frac{\mathrm{d} \lambda}{\lambda}
$$

converges strictly to an element $Q \in \operatorname{Fix}^{\mathbb{R}_{>0}}(\mathbb{J}, \overline{\mathcal{R}})$. Moreover,

$$
\tilde{p}_{0}(Q)=\lim _{i} \int_{0}^{\infty} \chi_{i}(\lambda) \sigma_{\lambda}\left(f_{0}\right) \frac{\mathrm{d} \lambda}{\lambda} \in \operatorname{Fix}^{\mathbb{R}}>0\left(\rrbracket_{0}, \overline{\mathcal{R}_{0}}\right) .
$$

Proof. As $f_{0} \in \mathcal{S}_{0}\left(T_{H} M\right)$, the same argument as in the proof of Lemma 8.12 gives $f_{j}, g_{j} \in \mathcal{R}_{0}$ with $f_{0}=\sum_{j=1}^{n} f_{j}^{*} * g_{j}$. Let $F_{j}, G_{j} \in \mathcal{R}$ be extensions of $f_{j}, g_{j}$ to $t>0$, which can be obtained as in the proof of Lemma 6.12 As $h:=f-\sum_{j=1} F_{j}^{*} *$ $G_{j} \in \mathcal{A}\left(\mathbb{T}_{H} M\right)$ vanishes at $t=0$, it follows from Lemma 7.12 that

$$
\int_{0}^{\infty} \chi_{i}(\lambda) \sigma_{\lambda}(h) \frac{\mathrm{d} \lambda}{\lambda}
$$

converges strictly to an element of the generalized fixed point algebra. As

$$
\int_{0}^{\infty} \chi_{i}(\lambda) \sigma_{\lambda}(f) \frac{\mathrm{d} \lambda}{\lambda}=\int_{0}^{\infty} \chi_{i}(\lambda) \sigma_{\lambda}(h) \frac{\mathrm{d} \lambda}{\lambda}+\sum_{j=1}^{n} \int_{0}^{\infty} \chi_{i}(\lambda) \sigma_{\lambda}\left(F_{j}^{*} * G_{j}\right) \frac{\mathrm{d} \lambda}{\lambda}
$$

and the operators on the right converge to $\left.\left|F_{j}\right\rangle\right\rangle\left\langle\left\langle G_{j}\right|\right.$, it follows that the left hand side converges strictly to a $Q \in \operatorname{Fix}^{\mathbb{R}>0}(\mathbb{J}, \overline{\mathcal{R}})$. As $h \in \operatorname{ker}\left(p_{0}\right)$, the decomposition above also shows that

$$
\left.\widetilde{p}_{0}(Q)=\sum_{j=1}^{n}\left|f_{j}^{*}\right\rangle\right\rangle\left\langle\left\langle g_{j}\right|=\lim _{i} \int_{0}^{\infty} \chi_{i}(\lambda) \sigma_{\lambda}\left(f_{0}\right) \frac{\mathrm{d} \lambda}{\lambda} .\right.
$$

THEOREM 9.15. The pseudodifferential extension of order zero from 42 for a filtered manifold $(M, H)$ embeds into the generalized fixed point algebra extension from (37) such that the following diagram commutes

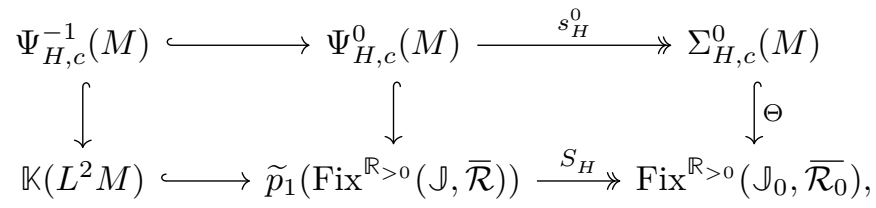

with $S_{H}=\widetilde{p}_{0} \circ\left(\widetilde{p}_{1}\right)^{-1}$. 
Proof. For the inclusion of $\Psi_{H, c}^{0}(M)$ in the generalized fixed point algebra, let $P \in \Psi_{H, c}^{0}(M)$. By Proposition 9.13 there is a function $f \in \mathcal{R}$ with $f_{0} \in \mathcal{S}_{0}\left(T_{H} M\right)$ and

$$
P-\int_{0}^{\infty} p_{1}\left(\sigma_{\lambda}(f)\right) \frac{\mathrm{d} \lambda}{\lambda} \in \mathrm{C}_{c}^{\infty}(M \times M) .
$$

By Lemma 9.14 $\int_{0}^{\infty} \chi_{i}(\lambda) \sigma_{\lambda}(f) \frac{\mathrm{d} \lambda}{\lambda}$ converges to an element $Q$ of the generalized fixed point algebra $\operatorname{Fix}^{\mathbb{R}>0}(\mathbb{J}, \overline{\mathcal{R}})$. Its image $\widetilde{p}_{1}(Q)$ in $\mathbb{B}\left(L^{2} M\right)$ is the unique bounded extension of the convolution operator associated with $\int_{0}^{\infty} p_{1}\left(\sigma_{\lambda}(f)\right) \frac{\mathrm{d} \lambda}{\lambda}$. Convolution by the kernel in $\mathrm{C}_{c}^{\infty}(M \times M)$ extends to a compact operator $K$. It follows that $K+Q$ is the unique continuous extension of $\mathrm{Op}(P)$ and belongs to the generalized fixed point algebra. Moreover, Lemma 9.14 also implies

$$
\left.\Theta\left(s_{H}^{0}(P)\right)=\int_{0}^{\infty} \sigma_{\lambda}\left(f_{0}\right) \frac{\mathrm{d} \lambda}{\lambda}=\sum_{j=1}^{n}\left|f_{j}\right\rangle\right\rangle\left\langle\left\langle g_{j}\right|=S_{H}(Q)=S_{H}(\mathrm{Op}(P)) .\right.
$$

Hence, the right square in 43 commutes. For $P \in \Psi_{H, c}^{-1}(M)$ the commutativity of the right square and exactness of the rows yields that $\mathrm{Op}(P)$ extends to a compact operator on $L^{2}(M)$.

Remark 9.16. In particular, we recover the result in $[\mathbf{D H 1 7}, 3.9]$ that $H$-pseudodifferential operators of order 0 extend to bounded operators on $L^{2}(M)$, while the ones of order -1 define compact operators.

As the left and middle vertical arrows in 43 are inclusions of *-algebras, the quotient map $\Theta: \Sigma_{H, c}^{0} \rightarrow \operatorname{Fix}^{\mathbb{R}>0}\left(\mathbb{J}_{0}, \overline{\mathcal{R}_{0}}\right)$ must be a *-homomorphism, too. Therefore, $\operatorname{Fix}^{\mathbb{R}>0}\left(\mathbb{J}_{0}, \overline{\mathcal{R}_{0}}\right)$ is the $\mathrm{C}^{*}$-completion of $\Sigma_{H, c}^{0}(M)$.

Corollary 9.17. Let $\mathrm{C}^{*}\left(\Psi_{H, c}^{0}(M)\right)$ be the $\mathrm{C}^{*}$-closure of $\Psi_{H, c}^{0}(M)$ inside $\mathbb{B}\left(L^{2} M\right)$. Then $\mathrm{C}^{*}\left(\Psi_{H, c}^{0}(M)\right)$ is isomorphic to $\mathrm{Fix}^{\mathbb{R}}{ }^{\mathbb{0}}(\mathbb{J}, \overline{\mathcal{R}})$. There is a short exact sequence of $\mathrm{C}^{*}$-algebras

$$
\mathbb{K}\left(L^{2} M\right) \longleftrightarrow \mathrm{C}^{*}\left(\Psi_{H, c}^{0}(M)\right) \stackrel{S_{H}}{\longrightarrow} \mathrm{C}^{*}\left(\Sigma_{H, c}^{0}(M)\right),
$$

where $S_{H}$ extends the principal cosymbol map $s_{H}^{0}: \Psi_{H, c}^{0}(M) \rightarrow \Sigma_{H, c}^{0}(M)$.

Proof. We show that $\mathrm{C}^{*}\left(\Psi_{H, c}^{0}(M)\right)=\widetilde{p}_{1}\left(\mathrm{Fix}^{\mathbb{R}}>0\left(\mathbb{J}_{0}, \overline{\mathcal{R}}\right)\right)$. The $\mathrm{C}^{*}$-algebra of $H$-pseudodifferential operators of order 0 is contained in $\widetilde{p}_{1}\left(\operatorname{Fix}^{\mathbb{R}>0}\left(\mathbb{J}_{0}, \overline{\mathcal{R}}\right)\right)$ by Theorem 9.15

For the converse, note first that $\mathbb{K}\left(L^{2} M\right) \subset \mathrm{C}^{*}\left(\Psi_{H, c}^{0}(M)\right)$. This holds as $\Psi_{H, c}^{0}(M)$ contains the kernels in $\mathrm{C}_{c}^{\infty}(M \times M)$ and these generate the compact operators on $L^{2}(M)$. Now, let $f, g \in \mathcal{R}$. Let $u \in \Sigma_{H, c}^{0}(M)$ be the inverse of $\left.\left|f_{0}\right\rangle\right\rangle\left\langle\left\langle g_{0}\right| \in \mathcal{K}^{0}\left(T_{H} M\right)\right.$ under the map $\Theta$ in Lemma 9.11. Since the principal cosymbol map is surjective, there is a $P \in \Psi_{H, c}^{0}(M)$ with $s_{H}^{0}(P)=u$. Then the operator

$$
\widetilde{p}_{1}(|f\rangle\rangle\langle\langle g|)=\widetilde{p}_{1}(|f\rangle\rangle\langle\langle g|)-\mathrm{Op}(P)+\mathrm{Op}(P)
$$

is contained in $\mathrm{C}^{*}\left(\Psi_{H, c}^{0}(M)\right)$. This is because $\mathrm{Op}(P)$ is and $\widetilde{p}_{1}(|f\rangle\rangle\langle\langle g|)-\mathrm{Op}(P) \in$ $\mathbb{K}\left(L^{2} M\right)$ as the diagram in 43$)$ commutes. The $\mathrm{C}^{*}$-algebra $\operatorname{Fix}^{\mathbb{R}>0}(\mathbb{J}, \overline{\mathcal{R}})$ is generated by $|f\rangle\rangle\langle\langle g|$ with $f, g \in \mathcal{R}$. Thus, the result follows.

The convolution algebra $\operatorname{Ess}_{H, c}^{0}(M)$ can be completed into a $\mathrm{C}^{*}$-algebra. Consider the ${ }^{*}$-representations $\lambda_{x}$ for $x \in M$ given by the corresponding convolution operators, that is,

$$
\lambda_{x}(u) f=u_{x} * f \quad \text { for } u \in \operatorname{Ess}_{H, c}^{0}(M) \text { and } f \in \mathcal{S}(G(x)) .
$$


To see that these extend to bounded operators on $L^{2}(G(x))$, recall that by Proposition 9.10 there is a $w \in \mathcal{K}^{0}\left(T_{H} M\right)$ such that $u-w \in \mathrm{C}_{\mathrm{cp}}^{\infty}\left(T_{H} M\right)$. Let $\chi \in \mathrm{C}_{c}^{\infty}\left(T_{H} M\right)$ be such that $\chi \cdot u=u$. Then one can write $u=\chi(u-w)+\chi w$. The first part is in $\mathrm{C}_{c}^{\infty}\left(T_{H} M\right)$, so convolution with it extends to a bounded operator. As $w_{x}$ extends to a continuous operator $L^{2}(G(x)) \rightarrow L^{2}(G(x))$ by Theorem 8.18 also convolution with $\chi_{x} w_{x}$ extends to a bounded operator by Chr88, 2.10]. In fact, using the compact support in the base space, the proof of $[\mathbf{C h r 8 8}, 2.10]$ yields a $C>0$ with

$$
\left\|\lambda_{x}(u)\right\| \leq\|\chi(u-w)\|+C\|w\| \text { for all } x \in M \text {. }
$$

Thus, one can take the completion of $\operatorname{Ess}_{H, c}^{0}(M)$ with respect to the norm

$$
\|u\|:=\sup _{x \in M}\left\|\lambda_{x}(u)\right\| \quad \text { for } u \in \operatorname{Ess}_{H, c}^{0}(M) .
$$

Denote the resulting $\mathrm{C}^{*}$-algebra by $\mathrm{C}^{*}\left(\operatorname{Ess}_{H, c}^{0}(M)\right)$. The homomorphism

$$
\Theta: \operatorname{Ess}_{H, c}^{0}(M) / \mathrm{C}_{c}^{\infty}\left(T_{H} M\right)=\Sigma_{H, c}^{0}(M) \rightarrow \operatorname{Fix}^{\mathbb{R}>0}\left(\mathbb{J}_{0}, \overline{\mathcal{R}}\right)
$$

has dense image. Therefore, one obtains an extension of $\mathrm{C}^{*}$-algebras

$$
\mathrm{C}^{*}\left(T_{H} M\right) \longleftrightarrow \mathrm{C}^{*}\left(\operatorname{Ess}_{H, c}^{0}(M)\right) \longrightarrow \mathrm{Fix}^{\mathbb{R}>0}\left(\mathbb{J}_{0}, \overline{\mathcal{R}}\right)
$$

Remark 9.18. For a step 1 filtration, the short exact sequence above corresponds under Fourier transform to the disk bundle extension

$$
\mathrm{C}_{0}\left(T^{*} M\right) \longleftrightarrow \mathrm{C}_{0}\left(B^{*} M\right) \longrightarrow \mathrm{C}_{0}\left(S^{*} M\right) .
$$

Denote by $\left.\Downarrow_{H, c}^{0}(M)\right|_{[0,1]}$ the *-algebra obtained by restricting to $[0,1]$. It can be completed into a $\mathrm{C}^{*}$-algebra using the left regular representations $\mathrm{Op}_{t}$ for $t>0$ in the following way. Let $\left.\mathbb{P} \in \Downarrow_{H, c}^{0}(M)\right|_{[0,1]}$. Using Proposition 9.13 one can find an element $T \in \operatorname{Fix}^{\mathbb{R}_{>0}}(\mathbb{J}, \overline{\mathcal{R}})$ with associated distributions $K=\left(K_{t}\right)$ such that $\mathbb{P}-K \in \mathrm{C}^{\infty}\left(\left.\mathbb{T}_{H} M\right|_{[0,1]}\right)$. Furthermore, there is a cutoff function $\chi \in \mathrm{C}_{c}^{\infty}\left(\mathbb{T}_{H} M\right)$ that is 1 in a neighbourhood of the unit space such that $\chi \mathbb{P}=\mathbb{P}$. Then $\chi(\mathbb{P}-K) \in$ $\mathrm{C}_{c}^{\infty}\left(\left.\mathbb{T}_{H} M\right|_{[0,1]}\right)$. Moreover, $\chi_{t} K_{t}$ extends to bounded operator for each $t>0$ as $K_{t}$ does and $\left(1-\chi_{t}\right) K_{t}$ is supported away from the diagonal and is, therefore, smooth and compactly supported. For $t=0$, this was discussed above. It follows that

$$
\left\|\mathrm{Op}_{t}(\mathbb{P})\right\| \leq\|\chi(\mathbb{P}-K)\|+\|\chi K\| \quad \text { for all } 0<t \leq 1
$$

Thus, one can complete $\left.\Downarrow_{H, c}^{0}(M)\right|_{[0,1]}$ with respect to

$$
\|\mathbb{P}\|:=\max \left\{\sup _{0<t \leq 1}\left\|\mathrm{Op}_{t}(\mathbb{P})\right\|,\left\|\mathbb{P}_{0}\right\|\right\} \quad \text { for }\left.\mathbb{P} \in \Psi_{H, c}^{0}(M)\right|_{[0,1]} .
$$

We will denote the $\mathrm{C}^{*}$-completion by $\mathrm{C}^{*}\left(\Psi_{H, c}^{0}(M)\right)$. As elements of $\Psi_{H, c}^{0}(M)$ are continuous families of distributions, $\mathrm{C}^{*}\left(\Psi_{H, c}^{0}(M)\right)$ is a continuous field of $\mathrm{C}^{*}$-algebras over $[0,1]$. Note that $\mathrm{C}^{*}\left(\left.\mathbb{T}_{H} M\right|_{[0,1]}\right)$ is an ideal in $\mathrm{C}^{*}\left(\Psi_{H, c}^{0}(M)\right)$.

Lemma 9.19. The kernel of the homomorphism

$$
\mathbb{S}_{H}^{0}: \mathrm{C}^{*}\left(\Psi_{H, c}^{0}(M)\right) \rightarrow \mathrm{Fix}^{\mathbb{R}>0}\left(\mathbb{J}_{0}, \overline{\mathcal{R}_{0}}\right)
$$

induced by $\mathbb{P} \mapsto\left[\mathbb{P}_{0}\right]$ is $\mathrm{C}^{*}\left(\left.\mathbb{T}_{H} M\right|_{[0,1]}\right)$.

Proof. Since $\mathrm{C}^{*}\left(\left.\mathbb{T}_{H} M\right|_{[0,1]}\right)$ is generated by $\mathrm{C}_{c}^{\infty}\left(\left.\mathbb{T}_{H} M\right|_{[0,1]}\right)$ which is contained in the kernel of $\mathbb{S}_{H}^{0}$, the first inclusion follows. For the converse inclusion, note that the kernel is generated by $\left.\Downarrow_{H, c}^{-1}(M)\right|_{[0,1]}$. Let $\left.\mathbb{P} \in \Downarrow_{H, c}^{-1}(M)\right|_{[0,1]}$. Tracing back the construction in Proposition 9.13 one can take $f \in \mathcal{R}$ such that $f$ vanishes at $t=0$. It follows that the corresponding element $T \in \operatorname{Fix}^{\mathbb{R}>0}(\mathbb{J}, \overline{\mathcal{R}})$ defines a compact operator. Therefore, $\operatorname{Op}\left(\mathbb{P}_{t}\right) \in \mathbb{K}\left(L^{2} M\right)$ for all $t>0$. By assumption 
$\mathbb{P}_{0} \in \mathrm{C}_{c}^{\infty}\left(T_{H} M\right) \subset \mathrm{C}^{*}\left(T_{H} M\right)$ holds, so that $\mathbb{P}$ belongs pointwise to $\mathrm{C}^{*}\left(\mathbb{T}_{H} M\right)$. As $\mathrm{C}^{*}\left(\Downarrow_{H, c}^{0}(M)\right)$ is a continuous field, it follows $\mathbb{P} \in \mathrm{C}^{*}\left(\mathbb{T}_{H} M\right)$ by $\left.\operatorname{Dix} 77,10.4 .2\right]$.

\subsection{The calculus of Fischer, Ruzhansky and Fermanian-Kammerer}

Recall the operator-valued Fourier transform for a nilpotent Lie group $G$ described in Section 3.4 It maps a right-invariant operator on $L^{2}(G)$, like the model operators on the osculating groups, to a field of operators $\left\{a(\pi) \in \mathbb{B}\left(\mathcal{H}_{\pi}\right) \mid \pi \in \widehat{G}\right\}$. For an arbitrary filtered manifold, it might be inconvenient to work with it as the osculating groups and, therefore, their representation theory can change from point to point.

However, when viewing a fixed graded Lie group $G$ as a filtered manifold as in Example 4.5 the operator-valued Fourier transform can be used to define a symbolic pseudodifferential calculus. This was carried out in [FR16. In [FFK17], homogeneous expansions for the symbols were defined. We give a short introduction to their calculus. The symbols in their calculus are fields of operators

$$
\left\{a(x, \pi): \mathcal{H}_{\pi}^{\infty} \rightarrow \mathcal{H}_{\pi} \mid x \in G, \pi \in \widehat{G}\right\} .
$$

Here, $\mathcal{H}_{\pi}^{\infty}$ are the smooth vectors in $\mathcal{H}_{\pi}$.

Remark 9.20. Note that FR16 uses a different convention for the Fourier transform

$$
\mathcal{F}(f)(\pi)=\int_{G} f(x) \pi(x)^{*} \mathrm{~d} x \quad \text { for } f \in L^{1}(G) .
$$

This leads to $\mathcal{F}(f * g)(\pi)=\mathcal{F}(g)(\pi) \mathcal{F}(f)(\pi)$ for $\pi \in \widehat{G}$ and $f, g \in L^{1}(G)$. In this case, $L^{\infty}\left(\widehat{G}, \mathbb{B}\left(\mathcal{H}_{\pi}\right)\right)$ is identified with the right von Neumann algebra $\operatorname{VN}_{R}(G)$. In particular, their operators have right convolution kernels.

The pseudodifferential calculus is defined in FR16 using a positive Rockland operator. Each $\pi \in \widehat{G}$ yields an infinitesimal representation $\mathrm{d} \pi$ of $\mathcal{U}(\mathfrak{g})$ on $\mathcal{H}_{\pi}^{\infty}$ (see [FR16, 1.7.3]). As in [FR16 we will write $\pi(P):=\mathrm{d} \pi(P)$ for right-invariant differential operators $P$ on $G$.

Definition 9.21 (FR16 4.1.1, 4.1.2]). Let $G$ be a homogeneous Lie group. A right-invariant differential operator $P$ on $G$ satisfies the Rockland condition if $\pi(P)$ is injective on $\mathcal{H}_{\pi}^{\infty}$ for all $\pi \in \widehat{G} \backslash\left\{\pi_{\text {triv }}\right\}$.

A right-invariant differential operator $P$ which is homogeneous of positive degree and satisfies the Rockland condition is called a Rockland operator.

Example 9.22. For $G=\mathbb{R}^{n}$ the Laplace operator $\Delta_{n}=\sum_{j=1}^{n} \partial_{j}^{2}$ is a Rockland operator. There is an isomorphism $\mathbb{R}^{n} \rightarrow \widehat{\mathbb{R}^{n}}, \xi \mapsto \pi_{\xi}$, given by $\pi_{\xi}(x)=e^{-i\langle\xi, x\rangle}$. One computes that $\pi_{\xi}\left(\partial_{j}\right)=-i \xi_{j}$. Hence, $\pi_{\xi}\left(\Delta_{n}\right)=-\|\xi\|^{2} \neq 0$ for $\xi \neq 0$.

Example 9.23 ([FR16, 4.1.8]). Let $G$ be a graded Lie group with weights $q_{1} \leq$ $q_{2} \leq \ldots \leq q_{n}$ and corresponding basis $X_{1}, \ldots, X_{n}$ of $\mathfrak{g}$. Let $q$ be a common multiple of the weights. Then the following operator is a Rockland operator

$$
\sum_{j=1}^{n}(-1)^{q / q_{j}} X_{j}^{2 q / q_{j}} .
$$

Example 9.24. Let $G=\mathbb{H}_{n}$ be the Heisenberg group with generators $X_{j}, Y_{j}, Z$ for $j=1, \ldots, n$ as in Example 3.2 The following operators and variants of them were also considered in $[$ Eps99, vE10a vE10b BvE14. For $\mu \in \mathbb{C}$ let

$$
P_{\mu}=\sum_{j=1}^{n}-X_{j}^{2}-Y_{j}^{2}+i \mu Z .
$$


In particular, we obtain for $\mu=0$ the sub-Laplacian $P_{0}=\sum_{j=1}^{n}-X_{j}^{2}-Y_{j}^{2}$ on the Heisenberg group. For $n=1$, this is the operator (1) considered in the introduction.

The computation of the coadjoint orbits in Example 3.31 yields representatives of the coadjoint orbits $\sum_{j=1}^{n} \alpha_{j} X_{j}^{*}+\beta_{j} Y_{j}^{*}$ for $(\alpha, \beta) \in \mathbb{R}^{2 n}$ and $\gamma Z^{*}$ for $\gamma \neq 0$. Note that $(\alpha, \beta)=(0,0)$ corresponds to the trivial representation under the Kirillov map.

In the first case, the corresponding representation $\chi_{\alpha, \beta}$ of $\mathbb{H}_{n}$ is one-dimensional and given by $\chi_{\alpha, \beta}(x, y, z)=e^{i(\langle\alpha, x\rangle+\langle\beta, y\rangle)}$ for $(x, y, z) \in \mathbb{H}_{n}$. One computes that $\chi_{\alpha, \beta}\left(P_{\mu}\right)=\|\alpha\|_{2}^{2}+\|\beta\|_{2}^{2} \neq 0$ whenever $(\alpha, \beta) \neq(0,0)$.

The representation of $H_{n}$ associated to $\gamma Z^{*}$ with $\gamma \neq$ is unitarily equivalent to a representation $\pi_{\gamma}: \mathbb{H}_{n} \rightarrow L^{2}\left(\mathbb{R}^{n}\right)$ as in [FR16. Sec. 6.2.1]. The smooth vectors are $\mathcal{S}\left(\mathbb{R}^{n}\right)$. It is computed in $[\mathbf{F R 1 6}, 6.2 .1]$ that infinitesimal representations are given by

$$
\begin{aligned}
\pi_{\gamma}\left(X_{j}\right) & =\sqrt{|\gamma|} \frac{\partial}{\partial u_{j}} \quad \text { for } j=1, \ldots, n, \\
\pi_{\gamma}\left(Y_{j}\right) & = \pm \sqrt{|\gamma|} i u_{j} \quad \text { for } j=1, \ldots, n, \\
\pi_{\gamma}(Z) & =i \gamma .
\end{aligned}
$$

Here, \pm corresponds to the sign of $\gamma$. Consequently, $P_{\mu}$ satisfies the Rockland condition if and only if

$$
\pi_{\gamma}\left(P_{\mu}\right)=\gamma\left(-\Delta_{n}+|u|^{2} \pm \mu I\right)
$$

is injective on $\mathcal{S}\left(\mathbb{R}^{n}\right)$. It was already observed in Eps99, Sec. 8] that the quantum harmonic oscillator $-\Delta_{n}+|u|^{2}$ shows up. In Eps99 Sec. 9] it is proved that it has pure point spectrum and its spectrum is given by $\left\{n+2 l \mid l \in \mathbb{N}_{0}\right\}$. The corresponding eigenfunctions are Schwartz. Therefore, $P_{\mu}$ satisfies the Rockland condition if and only if $\mu \notin\left\{n+2 l \mid l \in \mathbb{N}_{0}\right\} \cup\left\{-n-2 l \mid l \in \mathbb{N}_{0}\right\}$.

Remark 9.25. The existence of a positive Rockland operator on a homogeneous Lie group is equivalent to the group being (up to rescaling) graded (see FR16 4.1.3, 4.1.8]).

Remark 9.26. The Helffer-Nourrigat Theorem [HN79] states that a right-invariant homogeneous differential operator on a graded Lie group satisfies the Rockland condition if and only if it is hypoelliptic.

From now on, let $\mathfrak{R}$ be a fixed positive Rockland operator of homogeneous degree $q$ on $G$. It will play the role of the Laplace operator on $\mathbb{R}^{n}$.

Using the positive Rockland operator and its functional calculus, the Sobolev spaces $L_{s}^{2}(G)$ for $s \in \mathbb{R}$ are defined in [FR16, 4.4.2]. Moreover, the operatorvalued Fourier transform extends to a Fourier transform between right-invariant operators in $\mathbb{B}\left(L_{a}^{2}(G), L_{b}^{2}(G)\right)$ for $a, b \in \mathbb{R}$ to a space of fields denoted by $L_{a, b}^{\infty}(\widehat{G})$, see [FR16, 5.1.21, 5.1.24]. The spaces of corresponding convolution kernels in $\mathcal{S}^{\prime}(G)$ are denoted by $\mathcal{K}_{a, b}(G)$.

The derivatives in the cotangent direction in the Euclidean calculus are replaced with difference operators $\Delta^{\alpha}$ for $\alpha \in \mathbb{N}_{0}^{n}$, which are defined in the following. This is based on the observation that the Euclidean Fourier transform intertwines $\partial^{\alpha}$ and with multiplication by $x^{\alpha}$. For $u \in \mathcal{S}^{\prime}(G)$ denote by $x^{\alpha} u$ for $\alpha \in \mathbb{N}_{0}^{n}$ the tempered distributions defined by

$$
\left\langle x^{\alpha} u, f\right\rangle=\left\langle u, x^{\alpha} f\right\rangle \quad \text { for } f \in \mathcal{S}(G) .
$$

Let $u \in \mathcal{K}_{a, b}(G)$ be a kernel such that $x^{\alpha} u \in \mathcal{K}_{a^{\prime}, b^{\prime}}(G)$ for some $a^{\prime}, b^{\prime} \in \mathbb{R}$. Then the difference operator $\Delta^{\alpha}$ is defined as in [FR16, 5.2.1] by

$$
\Delta^{\alpha} \widehat{u}(\pi):=\widehat{x^{\alpha} u}(\pi) \quad \text { for } \pi \in \widehat{G} .
$$


The following symbol classes are adapted to the notion of order induced by the dilations. Hence, the homogeneous degree $[\alpha]$ for $\alpha \in \mathbb{N}_{0}^{n}$ as in Definition 3.15 is used.

Definition 9.27 ( FR16, 5.2.11]). A field $\left\{a(x, \pi): \mathcal{H}_{\pi}^{\infty} \rightarrow \mathcal{H}_{\pi} \mid x \in G, \pi \in \widehat{G}\right\}$ is a symbol of order $m \in \mathbb{R}$ if for all $\alpha, \beta \in \mathbb{N}_{0}^{n}$, the field of operators $X_{x}^{\beta} \Delta^{\alpha} a(x, \pi)$ is defined on the smooth vectors and satisfies

$$
\sup _{(x, \pi) \in G \times \widehat{G}}\left\|X_{x}^{\beta} \Delta^{\alpha} a(x, \pi) \pi(I+\mathfrak{R})^{\frac{[\alpha]-m}{q}}\right\|_{\mathbb{B}\left(\mathcal{H}_{\pi}\right)}<\infty .
$$

Denote the class of symbols of order $m$ by $S^{m}$. For $a \in S^{m}$ and $\alpha, \beta \in \mathbb{N}_{0}^{n}$ set

$$
\|a\|_{S^{m}, \alpha, \beta}=\sup _{(x, \pi) \in G \times \widehat{G}}\left\|X_{x}^{\beta} \Delta^{\alpha} a(x, \pi) \pi(I+\mathfrak{R})^{\frac{[\alpha]-m}{q}}\right\|_{\mathbb{B}\left(\mathcal{H}_{\pi}\right)} .
$$

The smoothing symbols are $S^{-\infty}=\bigcap_{m \in \mathbb{R}} S^{m}$.

One can form asymptotic expansions of these symbols in the following sense.

Proposition 9.28 (|FR16, 5.5.1]). Let $\left\{a_{j}\right\}_{j \in \mathbb{N}_{0}}$ be a sequence of symbols $a_{j} \in S^{m_{j}}$ with $m_{j}$ strictly decreasing to $-\infty$ as $j \rightarrow \infty$. Then there is a symbol $a \in S^{m_{0}}$, unique modulo $S^{-\infty}$, such that

$$
a-\sum_{j=0}^{M} a_{j} \in S^{m_{M+1}} \quad \text { for all } M \in \mathbb{N} .
$$

In this case, one writes $a \sim \sum_{j=0}^{\infty} a_{j}$.

Proposition 9.29 ([FFK17, 5.2.12, 5.2.17]). The symbol classes have the following properties:

(i) $S^{m_{1}} \subset S^{m_{2}}$ for $m_{1}<m_{2}$.

(ii) Each differential operator $\sum c_{\alpha}(x) X^{\alpha}$ with coefficients $c_{\alpha} \in \mathrm{C}^{\infty}(G)$ is contained in $S^{m}$, where $m=\max \left\{[\alpha] \mid c_{\alpha} \neq 0\right\}$.

(iii) For $a \in S^{m}$ and $\alpha, \beta \in \mathbb{N}_{0}^{n}$ the symbol $X^{\beta} \Delta^{\alpha} a$ is contained in $S^{m-[\alpha]}$.

For $a \in S^{m}$ the following quantization formula is well-defined and yields a continuous operator $\operatorname{Op}(a): \mathcal{S}(G) \rightarrow \mathcal{S}(G)$ by [FR16 5.2.15]

$$
\operatorname{Op}(a) \varphi(x)=\int_{\widehat{G}} \operatorname{tr}(\pi(x) a(x, \pi) \widehat{\varphi}(\pi)) \mathrm{d} \mu(\pi) \quad \text { for } \varphi \in \mathcal{S}(G), x \in G .
$$

Let $\kappa_{x} \in \mathcal{S}^{\prime}(G)$ be the convolution kernel of the right-invariant operator whose Fourier transform is $\{a(x, \pi) \mid \pi \in \widehat{G}\}$. Then $\operatorname{Op}(a) \varphi(x)=\kappa_{x} * \varphi$ holds. Denote by $K_{a} \in \mathcal{S}^{\prime}(G \times G)$ the integral kernel of $\operatorname{Op}(a)$. It is formally given by $K_{a}(x, y)=$ $\kappa_{x}\left(y x^{-1}\right)$.

In the following, we will consider operators with compactly supported integral kernels. Let $S_{\mathrm{cp}}^{m}$ consist of all symbols $a \in S^{m}$ such that $\operatorname{Op}(a)$ has a compactly supported integral kernel. Set $S_{\mathrm{cp}}^{-\infty}=\bigcap_{m \in \mathbb{R}} S_{\mathrm{cp}}^{m}$.

The following properties for the symbols with compactly supported integral kernels follow from the respective properties for the symbol classes $S^{m}$ shown in FR16, 5.5.8, 5.5.12, 5.7.2, 5.4.9, 5.2.21].

Proposition 9.30. The pseudodifferential calculus has the following properties:

(i) Let $m_{1}, m_{2} \in \mathbb{R}$. For $A \in \mathrm{Op}\left(S_{\mathrm{cp}}^{m_{1}}\right), B \in \mathrm{Op}\left(S_{\mathrm{cp}}^{m_{2}}\right)$ the composition $A B$ lies in $\mathrm{Op}\left(S_{\mathrm{cp}}^{m_{1}+m_{2}}\right)$.

(ii) Let $m \in \mathbb{R}$. For $A \in \mathrm{Op}\left(S_{\mathrm{cp}}^{m}\right)$ the formal adjoint $A^{*}$ lies in $\mathrm{Op}\left(S_{\mathrm{cp}}^{m}\right)$.

(iii) $A \in \mathrm{Op}\left(S_{\mathrm{cp}}^{m}\right)$ extends to a bounded operator $L_{s}^{2}(G) \rightarrow L_{s-m}^{2}(G)$ for $s \in \mathbb{R}$.

(iv) $A \in \mathrm{Op}\left(S_{\mathrm{cp}}^{-\infty}\right)$ if and only if its integral kernel lies in $\mathrm{C}_{c}^{\infty}(G \times G)$. 
For the following lemma, see also [FFK17, 4.24].

Lemma 9.31. Let $A \in \mathrm{Op}\left(S_{\mathrm{cp}}^{m}\right)$ for $m<0$. Then $A$ extends to a compact operator on $L^{2}(G)$.

Proof. By Proposition 9.30, $A$ extends to a bounded operator $A: L^{2}(G) \rightarrow$ $L_{-m}^{2}(G)$. Let $\chi \in \mathrm{C}_{c}^{\infty}(G)$ be constant 1 on the support of $A$ in the $x$-direction and be supported in a compact subset $K \subset G$. The map $f \mapsto \chi \cdot f$ extends to a bounded operator $L_{-m}^{2}(G) \rightarrow H^{\frac{-m}{q_{n}}}(K)$ by [FFK17, 2.17], where $H^{\frac{-m}{q_{n}}}(K)$ denotes the Euclidean Sobolev space. By Rellich's Theorem the embedding

$$
H^{\frac{-m}{q_{n}}}(K) \hookrightarrow L^{2}\left(\mathbb{R}^{n}\right)=L^{2}(G)
$$

is compact as $-m / q_{n}>0$. Therefore, the composition $A: L^{2}(G) \rightarrow L^{2}(G)$ is compact.

Moreover, in [FFK17 classical pseudodifferential operators, which admit a homogeneous expansion of their symbol, are defined.

Definition 9.32 $(\mid$ FFK17 $, 4.1,4.20])$. Let $m \in \mathbb{R}$. A field $\left\{a(x, \pi): \mathcal{H}_{\pi}^{\infty} \rightarrow \mathcal{H}_{\pi} \mid\right.$ $x \in G, \pi \in \widehat{G}\}$ is a regular $m$-homogeneous symbol if

(i) $a(x, \lambda \cdot \pi)=\lambda^{m} a(x, \pi)$ for all $x \in G$ and almost all $\pi$ and $\lambda>0$,

(ii) for all $\alpha, \beta \in \mathbb{N}_{0}^{n}$, the field of operators $X_{x}^{\beta} \Delta^{\alpha} a(x, \pi)$ is defined on smooth vectors and satisfies

$$
\sup _{(x, \pi) \in G \times \widehat{G}}\left\|X_{x}^{\beta} \Delta^{\alpha} a(x, \pi) \pi(\mathfrak{R})^{\frac{[\alpha]-m}{q}}\right\|_{\mathbb{B}\left(\mathcal{H}_{\pi}\right)}<\infty
$$

Denote by $\dot{S}^{m}$ the class of all regular $m$-homogeneous symbols and by $\dot{S}_{c}^{m}$ the ones with compact support in the $x$-direction.

Example 9.33 ( FFK17, 4.3, 4.4]). For each $c \in \mathrm{C}_{c}^{\infty}(G)$ and multi-index $\alpha \in \mathbb{N}_{0}^{n}$, the symbol $c(x) \pi(X)^{\alpha}$ belongs to $\dot{S}_{c}^{[\alpha]}$.

In the Euclidean case, homogeneous symbols are cut off in a neighbourhood of the zero section to obtain actual elements of the symbol classes. This corresponds to the following procedure for graded Lie groups.

Proposition 9.34 ([FFK17, 4.6]). Let $\psi \in \mathrm{C}^{\infty}([0, \infty))$ be a cutoff function with $0 \leq \psi \leq 1$ and $\left.\psi\right|_{[0,1]} \equiv 0$ and $\left.\psi\right|_{[2, \infty)} \equiv 1$. For all $m \in \mathbb{R}$ there is a linear map $c_{m}: \dot{S}^{m} \rightarrow S^{m}$ given by $a(x, \pi) \mapsto a(x, \pi) \psi(\pi(\Re))$.

This allows to define a homogeneous expansion of symbols.

Proposition 9.35 ([FFK17, 4.14]). Let $\left\{a_{j}\right\}_{j \in \mathbb{N}_{0}}$ be a sequence of homogeneous symbols $a_{j} \in \dot{S}^{m_{j}}$ with $m_{j}$ strictly decreasing to $-\infty$ as $j \rightarrow \infty$. Then there is a symbol $a \in S^{m_{0}}$, unique modulo $S^{-\infty}$, such that

$$
\forall M \in \mathbb{N} \quad a(x, \pi)-\sum_{j=0}^{M} a_{j}(x, \pi) \psi(\pi(\Re)) \in S^{m_{M+1}} .
$$

Moreover, if $a \in S^{m}$ for $m<m_{0}$, it follows that $a_{0}=0$.

In this case, we also write $a \sim \sum a_{j}$. There is a well-defined principal symbol for these operators.

Definition 9.36 (FFK17, 4.17, 4.20]). Let $a \in S^{m}$ be a symbol that admits a homogeneous expansion $a \sim \sum_{j=0}^{\infty} a_{j}$ as above with $a_{j} \in \dot{S}^{m-j}$. The principal part of $\mathrm{Op}(a)$ is defined as $\operatorname{princ}_{m}(\mathrm{Op}(a)):=a_{0}$. 
A symbol $a \in S_{\mathrm{cp}}^{m}$ is a classical pseudodifferential symbol of order $m$ if it admits a homogeneous expansion $a \sim \sum_{j=0}^{\infty} a_{j}$ with $a_{j} \in \dot{S}^{m-j}$. Denote by $S_{\mathrm{cl}}^{m}$ the classical pseudodifferential symbols of order $m$ and by $\Psi_{\mathrm{cl}}^{m}=\mathrm{Op}\left(S_{\mathrm{cl}}^{m}\right)$ the corresponding operators.

Proposition 9.37 (FFK17, 4.19]). Let $A \in \mathrm{Op}\left(S^{m}\right)$ and $B \in \mathrm{Op}\left(S^{l}\right)$ be operators whose symbols have a homogeneous expansion as above. Then the symbols of $A B$ and $A^{*}$ admit homogeneous expansions as well. Moreover, the following holds

$$
\begin{aligned}
\operatorname{princ}_{m+l}(A B) & =\operatorname{princ}_{m}(A) \cdot \operatorname{princ}_{l}(B) . \\
\operatorname{princ}_{m}\left(A^{*}\right) & =\operatorname{princ}_{m}(A)^{*} .
\end{aligned}
$$

On the right hand side, the pointwise operations are used.

In particular, princ $_{0}$ is a ${ }^{*}$-homomorphism.

Lemma 9.38. For $m \in \mathbb{Z}$, there are short exact sequences

$$
\Psi_{\mathrm{cl}}^{m-1} \longleftrightarrow \Psi_{\mathrm{cl}}^{m} \stackrel{\text { princ }_{m}}{\longrightarrow} \dot{S}_{c}^{m}
$$

For $m=0$, it is a short exact sequence of ${ }^{*}$-algebras.

Proof. Except for surjectivity of the principal symbol map, exactness is clear. Let $a_{0} \in \dot{S}_{c}^{m}$ and $a(x, \pi):=a_{0}(x, \pi) \psi(\pi(\mathfrak{R})) \in S^{m}$ as in Proposition 9.34. Then princ $_{m}(\mathrm{Op}(a))=a_{0}$ holds. We need to adjust $a$ in a way such that its integral kernel $K_{a}$ is compactly supported. Let $R>0$ be such that the support of $a$ in the $x$-direction in contained in $B(0, R)$. Pick a $c \in \mathrm{C}_{c}^{\infty}(G)$ which is constant 1 on $B\left(0,2 \gamma R^{2}\right)$. Here, $\gamma$ is the constant from the homogeneous triangle inequality in Lemma 3.12 Denote by $M_{c}: L^{2}(G) \rightarrow L^{2}(G)$ the multiplication operator $\varphi \mapsto c \cdot \varphi$. It belongs to $\Psi_{\text {cl }}^{0}$ with symbol given by $c(x) \operatorname{id}_{\mathcal{H}_{\pi}}$. Let $Q:=\operatorname{Op}(a) M_{c}$. Its integral kernel $K_{a}(x, y) c(y)$ is compactly supported. Moreover, $Q$ belongs to $\Psi_{\mathrm{cl}}^{m}$ and

$$
\operatorname{princ}_{m}(Q)=\operatorname{princ}_{m}(\mathrm{Op}(a))-\operatorname{princ}_{m}(\mathrm{Op}(a)-Q) .
$$

We show that last term is zero. Let $\kappa$ denote the convolution kernel of $\operatorname{Op}(a)$. The convolution kernel of $\mathrm{Op}(a)-Q$ is $\kappa_{x}(y)\left(1-c\left(y^{-1} x\right)\right)$. This is only non-zero if $\left\|y^{-1} x\right\| \geq 2 \gamma R^{2}$ and $\|x\|<R$. The homogeneous triangle inequality implies that $\|y\| \geq R$. But the $\kappa_{x}$ are smooth outside zero by [FR16, 5.4.1]. Therefore, $\mathrm{Op}(a)-Q$ is a smoothing operator and its principal symbol vanishes.

We compare the extension in 46 for $m=0$ to the short exact sequence of generalized fixed point algebras. First, we show that the $\mathrm{C}^{*}$-algebra $\mathrm{C}^{*}\left(\dot{S}_{c}^{0}\right)$ generated by regular 0-homogeneous symbols defined as in $\mathbf{F F K 1 7}$ is $\operatorname{Fix}^{\mathbb{R}>0}\left(\mathbb{J}_{0}, \overline{\mathcal{R}_{0}}\right)$. Recall that this generalized fixed point algebra is a continuous field of $\mathrm{C}^{*}$-algebras over $G$ with fibres $\operatorname{Fix}^{\mathbb{R}>0}\left(J_{G}, \overline{\mathcal{R}_{G}}\right)$ by Proposition 7.8

Definition 9.39. FFK17, 5.1, 5.5] The *-algebra of invariant, 0-homogeneous symbols $\tilde{S}^{0}$ consists of all $\left\{a(\pi): \mathcal{H}_{\pi}^{\infty} \rightarrow \mathcal{H}_{\pi}^{\infty} \mid \pi \in \widehat{G}\right\}$ that satisfy

(i) $a(\lambda \cdot \pi)=a(\pi)$ for almost all $\pi \in \widehat{G}$ and $\lambda>0$,

(ii) $\sup _{\pi \in \widehat{G}}\left\|\Delta^{\alpha} a(\pi) \pi(\Re)^{\frac{[\alpha]}{q}}\right\|_{\mathbb{B}\left(\mathcal{H}_{\pi}\right)}<\infty$ for all $\alpha \in \mathbb{N}_{0}^{n}$.

The $\mathrm{C}^{*}$-algebra of invariant 0-homogeneous symbols $\mathrm{C}^{*}\left(\tilde{S}^{0}\right)$ is the closure of $\tilde{S}^{0}$ with respect to

$$
\|a\|=\sup _{\pi \in \widehat{G} / \mathbb{R}_{>0}}\|a(\pi)\|
$$

Lemma 9.40. The ${ }^{*}$-algebra $\mathcal{K}^{0}(G)$ of kernels of type 0 is isomorphic to $\tilde{S}^{0}$ under Fourier transform. Moreover, $\operatorname{Fix}^{\mathbb{R}>0}\left(J_{G}, \overline{\mathcal{R}_{G}}\right)$ is isomorphic to $\mathrm{C}^{*}\left(\tilde{S}^{0}\right)$. 
Proof. By [FFK17, 5.3] the kernels of type 0 correspond exactly to the invariant, 0-homogeneous symbols under Fourier transform. By uniqueness of the $\mathrm{C}^{*}$-completion, $\mathrm{C}^{*}\left(\tilde{S}^{0}\right)$ is isomorphic to the $\mathrm{C}^{*}$-algebra generated by kernels of type 0 . This is $\operatorname{Fix}^{\mathbb{R}_{>0}}\left(J_{G}, \overline{\mathcal{R}_{G}}\right)$ by Corollary 8.20 .

Identifying the space of 0-homogeneous symbols with compact support in the $x$-direction $\dot{S}_{c}^{0}$ with $\mathrm{C}_{c}^{\infty}\left(G, \tilde{S}^{0}\right)$ as in the proof of $[$ FFK17, 5.9], yields the following result.

Corollary 9.41. The ${ }^{*}$-algebra of 0 -homogeneous symbols $\dot{S}_{c}^{0}$ is isomorphic under inverse Fourier transform to a dense ${ }^{*}$-subalgebra of $\operatorname{Fix}^{\mathbb{R}_{>0}}\left(\mathbb{J}_{0}, \overline{\mathcal{R}_{0}}\right)$.

To compare the sequence of generalized fixed point algebras to the order zero pseudodifferential extension from $(46)$, we show that operators in $\widetilde{p}_{1}\left(\operatorname{Fix}^{\mathbb{R}>0}(\mathbb{J}, \overline{\mathcal{R}})\right)$ can be written as in 45 in terms of a symbol. By Lemma 7.10 and Lemma 9.14. for $f \in \mathcal{R}$ with $f_{0} \in \mathcal{S}_{0}\left(T_{H} G\right)$ the operators $T_{i}(f)$ given by

$$
T_{i}(f) \varphi(x)=\int_{0}^{\infty} \chi_{i}(\lambda) \lambda^{-Q} \int f(x, y, \lambda) \varphi(y) \mathrm{d} \nu(y) \frac{\mathrm{d} \lambda}{\lambda} \quad \text { for } \varphi \in L^{2}(G), x \in G,
$$

converge strictly to an element $T(f) \in \widetilde{p}_{1}\left(\operatorname{Fix}^{\mathbb{R}>0}(\mathcal{J}, \overline{\mathcal{R}})\right)$.

We start by considering a slightly different generalized fixed point algebra. The reason for this is that one has to understand the convolution kernels $\kappa_{x}$ as a family of right-invariant operators in order to take their Fourier transform. Let $B:=\mathrm{C}^{*}\left(T_{H} G\right) \otimes \mathrm{C}_{0}([0, \infty))$ with $\mathbb{R}_{>0}$-action given by

$$
\beta_{\lambda}(h)(x, v, t)=\lambda^{Q} h\left(x, \lambda \cdot v, \lambda^{-1} t\right) \quad \text { for } \lambda>0 \text { and } h \in \mathrm{C}_{c}\left(T_{H} G \times[0, \infty)\right) .
$$

For $t \geq 0$ let $\mathrm{ev}_{t}: B \rightarrow \mathrm{C}^{*}\left(T_{H} G\right)$ be the homomorphism induced by the restriction. Define an $\mathbb{R}_{>0}$-invariant ideal $J_{B}$ with a dense subset $\mathcal{R} \subset J_{B}$ by

$$
\begin{aligned}
J_{B} & =\bigcap_{x \in G} \operatorname{ker}\left(\pi_{\text {triv }} \circ q_{x} \circ \mathrm{ev}_{0}\right), \\
\mathcal{R}_{B} & =\left\{h \in \mathrm{C}_{c}^{\infty}\left([0, \infty), \mathcal{S}_{\mathrm{cp}}\left(T_{H} G\right)\right) \mid \int_{G} h(x, v, 0) \mathrm{d} v=0 \text { for all } x \in G\right\} .
\end{aligned}
$$

Similar arguments as in Lemma 7.5 and Theorem 7.6 show:

Lemma 9.42. The ${ }^{*}$-subalgebra $\mathcal{R}_{B} \subset J_{B}$ is square-integrable for the action $\beta$ of $\mathbb{R}_{>0}$. Furthermore, $\left(J_{B}, \overline{\mathcal{R}_{B}}\right)$ is a continuously square-integrable $\mathbb{R}_{>0}-\mathrm{C}^{*}$-algebra.

Hence, $\operatorname{Fix}^{\mathbb{R}_{>0}}\left(J_{B}, \overline{\mathcal{R}_{B}}\right)$ is defined. The evaluations at $t=1$ and $x \in G$ composed with the left regular representation $\mathrm{C}^{*}(G) \rightarrow \mathbb{B}\left(L^{2} G\right)$, yield strictly continuous representations

$$
\widetilde{\lambda}_{x}: \operatorname{Fix}^{\mathbb{R}>0}\left(J_{B}, \overline{\mathcal{R}_{B}}\right) \rightarrow \mathrm{VN}_{L}(G) .
$$

Lemma 9.43. For $h \in \mathcal{R}_{B}$ with $h_{0} \in \mathcal{S}_{0}\left(T_{H} G\right)$

$$
\int_{0}^{\infty} \chi_{i}(\lambda) \beta_{\lambda}(h) \frac{\mathrm{d} \lambda}{\lambda}
$$

converges strictly to an element of $\operatorname{Fix}^{\mathbb{R}>0}\left(J_{B}, \overline{\mathcal{R}_{B}}\right)$. Its image under $\widetilde{\lambda}_{x}$ is given by

$$
\widetilde{\lambda}_{x}(h) \varphi=\lim _{i}\left(\int_{0}^{\infty} \chi_{i}(\lambda) \lambda^{-Q} h\left(x, \lambda^{-1}(\cdot), \lambda\right) \frac{\mathrm{d} \lambda}{\lambda} * \varphi\right) \quad \text { for } \varphi \in L^{2}(G) .
$$

Proof. The first claim is proved as in Lemma 9.14. This uses that there is an isomorphism

$$
\Psi: \operatorname{ker}\left(\mathrm{ev}_{0}\right) \rightarrow \mathrm{C}_{0}\left(\mathbb{R}_{>0}\right) \otimes \mathrm{C}^{*}\left(T_{H} G\right)
$$

which is induced by $\Psi(h)(t)=t^{-Q_{e v}}(h)$. The action $\beta$ corresponds to $\tau \otimes 1$ under the isomorphism. Here, $\tau$ is induced by the action of $\mathbb{R}_{>0}$ on itself by scaling. 
The second claim follows from strict continuity of $\widetilde{\lambda}_{x}$ and the computation $\mathrm{ev}_{t} \circ \beta_{\lambda}(h)=\lambda^{Q} \mathrm{ev}_{\lambda^{-1} t}(h)$ for $\lambda, t>0$.

Corollary 9.44. For $f \in \mathcal{R}$ with $f_{0} \in \mathcal{S}_{0}\left(T_{H} G\right)$, there is a symbol $\left\{a_{f}(x, \pi) \in\right.$ $\left.\mathbb{B}\left(\mathcal{H}_{\pi}\right) \mid x \in G, \pi \in \widehat{G}\right\}$ such that $T(f)=\operatorname{Op}\left(a_{f}\right)$.

Proof. We show that one can write $T(f) \varphi(x)=\kappa_{x} * \varphi$ for a smooth family of kernels $\kappa_{x} \in \mathcal{S}^{\prime}(G)$ such that $\varphi \mapsto \kappa_{x} * \varphi$ extends to a bounded operator on $L^{2}(G)$ for all $x \in G$. This implies that one can apply Fourier transform to $\kappa_{x}$ and one obtains a symbol as above with $a_{f}(x, \pi)=\widehat{\kappa_{x}}(\pi)$.

Let $g:=f \circ \phi^{-1}$ where $\phi$ is the global chart from Example 5.4 Then for each $i \in I$, one can write $T_{i}(f) \varphi(x)=\kappa_{i, x} * \varphi$ with

$$
\kappa_{i, x}(v)=\int_{0}^{\infty} \chi_{i}(\lambda) \lambda^{-Q} g\left(x, \lambda^{-1} \cdot v, \lambda\right) \frac{\mathrm{d} \lambda}{\lambda} .
$$

We claim that the $\kappa_{i, x}$ converge to distributions $\kappa_{x} \in \mathcal{S}^{\prime}(G)$ whose convolution operators are bounded. Note that $g$ can be understood as an element of $\mathcal{R}_{B}$ with $g_{0} \in \mathcal{S}_{0}\left(T_{H} G\right)$. It follows from Lemma 9.43 that $\kappa_{i, x}$ converges in $\mathcal{S}^{\prime}(G)$ to the convolution kernel of $\widetilde{\lambda}_{x}(g)$. In particular, $a_{f}(x, \pi)$ is the strict limit of

$$
\int_{0}^{\infty} \chi_{i}(\lambda) \widehat{g}(x, \lambda \cdot \pi, \lambda) \frac{\mathrm{d} \lambda}{\lambda}
$$

as multipliers of $\mathbb{K}\left(\mathcal{H}_{\pi}\right)$.

Let $a_{0} \in \dot{S}_{c}^{0}$. It was discussed in Proposition 9.34 that $a_{0} \psi(\pi(\Re))$ is in $S^{0}$. Lemma 9.43 yields a different way to attach a symbol to $a_{0}$. By Corollary 9.41. there is a $h_{0} \in \mathcal{S}_{0}\left(T_{H} G\right)$ such that $a_{0}$ is the Fourier transform of

$$
\int_{0}^{\infty} \sigma_{\lambda}\left(h_{0}\right) \frac{\mathrm{d} \lambda}{\lambda} \in \mathcal{K}^{0}\left(T_{H} G\right) .
$$

Let $\omega \in \mathrm{C}_{c}^{\infty}([0, \infty))$ be a function with $\left.\omega\right|_{[0,1]} \equiv 1$ and $\left.\omega\right|_{[2, \infty)} \equiv 0$. Define $h \in \mathcal{R}_{B}$ by $h(x, v, t):=\omega(t) h_{0}(x, v)$. By Lemma 9.43 this yields a symbol $a_{h} \in$ $\mathrm{C}_{0}\left(G, L^{\infty}(\widehat{G})\right)$. We compare now the symbols $a_{0} \psi(\pi(\Re))$ and $a_{h}$. As a preparation, the following lemma is proved.

Lemma 9.45. Let $h_{0} \in \mathcal{S}_{0}\left(T_{H} G\right)$ and let $\omega \in \mathrm{C}_{c}^{\infty}([0, \infty))$ be a function with $\left.\omega\right|_{[0,1]} \equiv 1$ and $\left.\omega\right|_{[2, \infty)} \equiv 0$. Define $h \in \mathcal{R}_{B}$ by $h(x, v, t):=\omega(t) h_{0}(x, v)$. Let $a_{h}(x, \pi)$ be the Fourier transform of $\widetilde{\lambda}_{x}(h)$ and $a_{0}(x, \pi)$ the Fourier transform of

$$
\int_{0}^{\infty} \sigma_{\lambda}\left(h_{0}\right) \frac{\mathrm{d} \lambda}{\lambda}
$$

Then for all $m>0$, there exists a constant $C_{m}>0$ with

$$
\left\|\left(a_{0}(x, \pi)-a_{h}(x, \pi)\right) \psi(\pi(\mathfrak{R}))(1+\pi(\mathfrak{R}))^{\frac{m}{q}}\right\| \leq C_{m}\left\|\widehat{h_{0}}\right\|_{S^{-m}, 0,0}
$$

for all $x \in G$ and almost all $\pi \in \widehat{G}$.

Proof. The symbols can be written as strict limits

$$
\begin{aligned}
& a_{0}(x, \pi)=\lim _{s} \int_{0}^{\infty} \chi_{i}(\lambda) \widehat{h_{0}}(x, \lambda \cdot \pi) \frac{\mathrm{d} \lambda}{\lambda}, \\
& a_{h}(x, \pi)=\lim _{s} \int_{0}^{\infty} \chi_{i}(\lambda) \omega(\lambda) \widehat{h_{0}}(x, \lambda \cdot \pi) \frac{\mathrm{d} \lambda}{\lambda},
\end{aligned}
$$

as multipliers of $\mathbb{K}\left(\mathcal{H}_{\pi}\right)$ for almost all $\pi \in \widehat{G}$. This implies that

$$
b_{i}(x, \pi):=\int_{0}^{\infty} \chi_{i}\left(\lambda^{-1}\right)(1-\omega(\lambda)) \widehat{h_{0}}(x, \lambda \cdot \pi) \frac{\mathrm{d} \lambda}{\lambda} \psi(\pi(\Re))(1+\pi(\mathfrak{R}))^{\frac{m}{q}}
$$


converges strongly to $b(x, \pi):=\left(a_{0}(x, \pi)-a_{h}(x, \pi)\right) \psi(\pi(\mathfrak{R}))(1+\pi(\mathfrak{R}))^{\frac{m}{q}}$ on $\mathcal{H}_{\pi}^{\infty}$. We show that $b_{i}(x, \pi)$ is a Cauchy sequence. As $\mathcal{H}_{\pi}^{\infty}$ is dense, this will imply that $b_{i}(x, \pi)$ converges to $b(x, \pi)$ in norm. For $j>i$ we estimate

$$
\begin{aligned}
& \left\|b_{j}(x, \pi)-b_{i}(x, \pi)\right\| \\
= & \left\|\int_{0}^{\infty}\left(\chi_{j}(\lambda)-\chi_{i}(\lambda)\right)(1-\omega(\lambda)) \widehat{h_{0}}(x, \lambda \cdot \pi) \psi(\pi(\Re))(1+\pi(\mathfrak{R}))^{\frac{m}{q}} \frac{\mathrm{d} \lambda}{\lambda}\right\| \\
\leq & \sup _{t \geq 1}\left(\frac{1+t}{t}\right)^{\frac{m}{q}} \int_{0}^{\infty}\left(\chi_{j}(\lambda)-\chi_{i}(\lambda)\right)(1-\omega(\lambda)) \sup _{(x, \pi)}\left\|\widehat{h_{0}}(x, \lambda \cdot \pi) \pi(\mathfrak{R})^{\frac{m}{q}}\right\| \frac{\mathrm{d} \lambda}{\lambda} \\
\lesssim & \int_{0}^{\infty}\left(1-\chi_{i}(\lambda)\right) \frac{1-\omega(\lambda)}{\lambda^{m}} \sup _{(x, \pi)}\left\|\widehat{h_{0}}(x, \lambda \cdot \pi)(\lambda \cdot \pi)(\mathfrak{R})^{\frac{m}{q}}\right\| \frac{\mathrm{d} \lambda}{\lambda} \\
\lesssim & \sup _{t \geq 0}\left(\frac{t}{1+t}\right)^{\frac{m}{q}} \int_{0}^{\infty}\left(1-\chi_{i}(\lambda)\right) \frac{1-\omega(\lambda)}{\lambda^{m}} \sup _{(x, \pi)}\left\|\widehat{h_{0}}(x, \lambda \cdot \pi)(1+(\lambda \cdot \pi)(\mathfrak{R}))^{\frac{m}{q}}\right\| \frac{\mathrm{d} \lambda}{\lambda} \\
\lesssim & \left\|\widehat{h_{0}}\right\|_{S^{-m}, 0,0} \int_{0}^{\infty}\left(1-\chi_{i}(\lambda)\right) \frac{1-\omega(\lambda)}{\lambda^{m+1}} \mathrm{~d} \lambda .
\end{aligned}
$$

The integral converges to 0 as the dominated convergence theorem can be applied due to the assumptions on $\omega$. Note that $\widehat{h_{0}}(x, \pi)$ is a smoothing symbol by FR16. 5.2.21], so that $\|c\|_{S^{-m}, 0,0}<\infty$ for all $m>0$. Using the same estimates there is a constant $C_{m}>0$ such that $\left\|b_{i}(x, \pi)\right\| \leq C_{m}\left\|\widehat{h_{0}}\right\|_{S^{-m}, 0,0}$ for all $i \in I$ and $(x, \pi) \in G \times \widehat{G}$. As $b(x, \pi)$ is the norm limit of this net, the claim follows.

Remark 9.46. The same result holds when $\psi(\pi(\mathfrak{R}))(1+\pi(\mathfrak{R}))^{\frac{m}{q}}$ is replaced by $\pi(\mathfrak{R})^{\frac{m}{q}}$.

Lemma 9.47. Let $h_{0} \in \mathcal{S}_{0}\left(T_{H} G\right), h \in \mathcal{R}_{B}, a_{0}$ and $a_{h}$ be as in Lemma 9.45. Then $a_{0} \psi(\pi(\mathfrak{R}))-a_{h} \in S^{-\infty}$ holds.

Proof. Write $a_{0} \psi(\pi(\mathfrak{R}))-a=\left(a_{0}-a\right) \psi(\pi(\mathfrak{R}))-a(1-\psi)(\pi(\mathfrak{R}))$. We claim that both summands are smoothing symbols. Recall that a symbol $b$ is smoothing if for all $m>0$ and $\alpha, \beta \in \mathbb{N}_{0}^{n}$

$$
\sup _{(x, \pi)}\left\|X_{x}^{\beta} \Delta^{\alpha}\{b(x, \pi)\}(1+\pi(\Re))^{\frac{[\alpha]+m}{q}}\right\|<\infty .
$$

For $\left(a_{0}-a\right) \psi(\pi(\mathfrak{R}))$ consider first the case $\alpha=0$. Then the result follows by applying Lemma 9.45 to $X_{x}^{\beta} h_{0} \in \mathcal{S}_{0}\left(T_{H} G\right)$. For arbitrary $\alpha \in \mathbb{N}_{0}^{n}$, the Leibniz rule for difference operators [FFK17, (3.1)] yields

$$
\Delta^{\alpha}\left\{\left(a_{0}-a\right)(x, \pi) \psi(\pi(\Re))\right\}=\sum_{\left[\alpha_{1}\right]+\left[\alpha_{2}\right]=[\alpha]}\left[\Delta^{\alpha_{1}}\left(a_{0}-a\right)(x, \pi)\right]\left[\Delta^{\alpha_{2}} \psi(\pi(\Re))\right] .
$$

For $\alpha_{2} \neq 0$, it is shown in [FFK17, 4.8] that

$$
\sup _{\pi}\left\|\pi(\Re) \frac{-m-\left[\alpha_{1}\right]}{q} \Delta^{\alpha_{2}} \psi(\pi(\Re))(1+\pi(\mathfrak{R}))^{\frac{m+[\alpha]}{q}}\right\|<\infty .
$$

Applying Remark 9.46 and Lemma 9.45 to $X_{x}^{\beta} v^{\alpha_{1}} h_{0}$ yields

$$
\sup _{(x, \pi)}\left\|X^{\beta} \Delta^{\alpha_{1}}\left(a_{0}-a\right)(x, \pi) \pi(\mathfrak{R})^{\frac{m+\left[\alpha_{1}\right]}{q}}\right\|<\infty .
$$

For $\alpha_{2}=0$, Lemma 9.45 is applied to $X_{x}^{\beta} v^{\alpha} h_{0} \in \mathcal{S}_{0}\left(T_{H} G\right)$.

Consider now the symbol $a(1-\psi)(\pi(\Re))$. As $(1-\psi)$ is supported in $[0,2]$ and $(1+t)^{\frac{[\alpha]+m}{q}}$ is bounded on this subset, it suffices to show for all $\alpha, \beta \in \mathbb{N}_{0}^{n}$ that

$$
\sup _{(x, \pi)}\left\|X_{x}^{\beta} \Delta^{\alpha} a(x, \pi)\right\|<\infty .
$$


For $\alpha=0$, this follows from Corollary 9.44 applied to $X_{x}^{\beta} h$. For $\alpha \neq 0$, the net

$$
\int_{0}^{\infty} \chi_{i}(\lambda) \omega(\lambda) \lambda^{[\alpha]}\left(\widehat{X_{x}^{\beta} v^{\alpha}} h_{0}\right)(x, \lambda \cdot \pi) \frac{\mathrm{d} \lambda}{\lambda}
$$

is Cauchy in $\mathrm{C}_{0}\left(G, L^{\infty}(\widehat{G})\right)$. This follows from $\omega(\lambda) \lambda^{[\alpha]} \leq \omega(\lambda) 2^{[\alpha]}$ and Corollary 9.44 applied to $X_{x}^{\beta} v^{\alpha} h_{0}$. Then $X_{x}^{\beta} \Delta^{\alpha}$ is the limit of this net as the respective convolution kernels converge in $\mathcal{S}^{\prime}(G)$.

THEOREM 9.48. Let $G$ be a graded Lie group. The order zero pseudodifferential extension from Lemma 9.38 embeds into the generalized fixed point algebra extension for $G$ such that the following diagram commutes

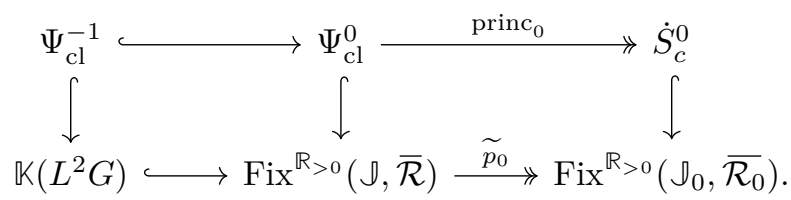

Proof. Every operator in $\Psi_{\mathrm{cl}}^{-1}$ extends to a compact operator on $L^{2}(G)$ by Lemma 9.31 Let $A$ be a classical pseudodifferential operator of order zero with principal symbol $a_{0} \in \dot{S}_{c}^{0}$. Let $Q \in \Psi_{\text {cl }}^{0}$ be the element constructed in the proof of Lemma 9.38 with $\operatorname{princ}_{0}(Q)=a_{0}$. Recall that $Q=\operatorname{Op}\left(a_{0}(x, \pi) \psi(\pi(\Re))\right) M_{c}$ for a certain $c \in \mathrm{C}_{c}^{\infty}(G)$. In the following we show that there is an element $T \in$ $\widetilde{p}_{1}\left(\operatorname{Fix}^{\mathbb{R}>0}(\mathbb{J}, \overline{\mathcal{R}})\right)$ with $\widetilde{p}_{0}(T)=a_{0}$ and $Q-T \in \mathbb{K}\left(L^{2} G\right)$. Once is this established, writing

$$
A=A-Q+Q-T+T
$$

shows that $A$ lies in $\widetilde{p}_{1}\left(\operatorname{Fix}^{\mathbb{R}_{>0}}(\mathbb{J}, \overline{\mathcal{R}})\right)$ as $A-Q$ has order -1 since its principal symbol vanishes. In particular, $A-Q$ is compact by Lemma 9.31. The above decomposition also shows that

$$
\tilde{p}_{0}(A)=\tilde{p}_{0}(T)=a_{0}=\operatorname{princ}_{0}(A)
$$

so that the diagram in 47 commutes. To construct $T$, let $h_{0} \in \mathcal{S}_{0}\left(T_{H} G\right)$ be such that $a_{0}$ is the Fourier transform of

$$
\int_{0}^{\infty} \sigma_{\lambda}\left(h_{0}\right) \frac{\mathrm{d} \lambda}{\lambda} .
$$

Let $h \in \mathcal{R}_{B}$ and $a_{h}$ be as in Lemma 9.45 and $g(x, v, t):=h(x, v, t) c(t \cdot v)$. Then $f:=g \circ \phi^{-1}$ belongs to $\mathcal{R}$, where $\phi$ is the global coordinate chart from Example 5.4 It satisfies $f(x, y, t)=(h \circ \phi)(x, y, t) c(y)$ for $t>0$. Corollary 9.44 implies that

$$
Q-T(f)=\operatorname{Op}\left(a_{0} \psi(\pi(\Re))-a_{h}\right) M_{c} .
$$

This is a compact operator as its convolution kernel is smooth by Lemma 9.47 and compactly supported.

Denote by $\mathrm{C}^{*}\left(\Psi_{\mathrm{cl}}^{0}\right)$ the closure of the ${ }^{*}$-algebra $\Psi_{\mathrm{cl}}^{0}$ in $\mathbb{B}\left(L^{2} G\right)$. The same arguments as in the proof of Corollary 9.17 give the following result.

Corollary 9.49. The $\mathrm{C}^{*}$-algebra $\mathrm{C}^{*}\left(\Psi_{\mathrm{cl}}^{0}\right)$ generated by classical order zero pseudodifferential operators on a graded Lie group $G$ is isomorphic to $\operatorname{Fix}^{\mathbb{R}}>0(\mathbb{J}, \overline{\mathcal{R}})$. There is an extension of $\mathrm{C}^{*}$-algebras

$$
\mathbb{K}\left(L^{2} G\right) \longleftrightarrow \mathrm{C}^{*}\left(\Psi_{\mathrm{cl}}^{0}\right) \stackrel{S_{H}}{\longrightarrow} \mathrm{C}^{*}\left(\dot{S}_{c}^{0}\right),
$$

such that $S_{H}$ extends the principal symbol map princ ${ }_{0}: \Psi_{\mathrm{cl}}^{0} \rightarrow \dot{S}_{c}^{0}$. 


\section{CHAPTER 10}

\section{Saturatedness and Morita equivalence}

In this section, we will show that $(\mathbb{J}, \overline{\mathcal{R}})$ and $\left(\mathbb{J}_{0}, \overline{\mathcal{R}_{0}}\right)$ are saturated for the zoom action of $\mathbb{R}_{>0}$. Therefore, for each filtered manifold $(M, H)$ the $\mathrm{C}^{*}$-algebras of order zero pseudodifferential operators $\operatorname{Fix}^{\mathbb{R}>0}(\mathbb{J}, \overline{\mathcal{R}})$ and principal cosymbols $\operatorname{Fix}\left(\mathbb{J}_{0}, \overline{\mathcal{R}_{0}}\right)$ are Morita-Rieffel equivalent to $\mathrm{C}_{\mathrm{r}}^{*}\left(\mathbb{R}_{>0}, \mathbb{J}\right)$ and $\mathrm{C}_{\mathrm{r}}^{*}\left(\mathbb{R}_{>0}, \mathbb{J}_{0}\right)$, respectively. For the Euclidean scalings this is a result of $\mathbf{D S 1 4}$.

\subsection{Graded Lie groups}

First, consider a homogeneous Lie group $G$. Recall the sequence of open, dilation invariant subsets of $\widehat{G} \backslash\left\{\pi_{\text {triv }}\right\}$ found in $(12)$ :

$$
\emptyset=V_{0} \subset V_{1} \subset V_{2} \subset \ldots \subset V_{m}=\widehat{G} \backslash\left\{\pi_{\text {triv }}\right\},
$$

where $\Lambda_{i}=V_{i} \backslash V_{i-1}$ are Hausdorff for all $i=1, \ldots, m$. Moreover, the induced $\mathbb{R}_{>0}$-action on each $\Lambda_{i}$ is free and proper by Proposition 3.35 There is a corresponding increasing sequence of closed, dilation invariant ideals in $\mathrm{C}^{*}(G)$

$$
0=J_{0} \triangleleft J_{1} \triangleleft J_{2} \triangleleft \ldots \triangleleft J_{m}=J_{G}
$$

which is given by

$$
J_{i}=\left\{f \in \mathrm{C}^{*}(G) \mid \widehat{\pi}(f)=0 \text { for } \pi \in \widehat{G} \backslash V_{i}\right\} .
$$

In this section, it will be shown that the subquotients $J_{i} / J_{i-1}$ of the filtration in 48 define continuous fields of $\mathrm{C}^{*}$-algebras over $\Lambda_{i}$, respectively. This will allow us to prove, using Corollary 2.22, that the generalized fixed point algebra of the dilation action on $J_{G}$ is Morita-Rieffel equivalent to the crossed product $\mathrm{C}_{\mathrm{r}}^{*}\left(\mathbb{R}_{>0}, J_{G}\right)$.

In BBL16 Pedersen's fine stratification Ped89 is used to obtain a similar sequence of increasing ideals, where the respective subquotients are even isomorphic to trivial fields $\mathrm{C}_{0}\left(\tilde{\Lambda}_{i}, \mathbb{K}\left(\mathcal{H}_{i}\right)\right)$ for some finite- or infinite-dimensional Hilbert spaces $\mathcal{H}_{i}$. For our purposes the coarse stratification suffices.

Proposition 10.1. Each subquotient $J_{i} / J_{i-1}$ for $i=1, \ldots, m$ is isomorphic to a continuous field of $\mathrm{C}^{*}$-algebras over $\Lambda_{i}$ with a unique dense, relatively continuous and complete subset $\mathcal{R}_{i}$ for the induced $\mathbb{R}_{>0}$-action. Furthermore, $\left(J_{i} / J_{i-1}, \mathcal{R}_{i}\right)$ is saturated.

Proof. The subquotient $J_{i} / J_{i-1}$ has Hausdorff spectrum as

$$
\widehat{J_{i} / J_{i-1}} \cong \widehat{J_{i} \backslash \widehat{J_{i-1}}} \cong V_{i} \backslash V_{i-1}=\Lambda_{i}
$$

Therefore, $J_{i} / J_{i-1}$ is isomorphic to a continuous field of $\mathrm{C}^{*}$-algebras over $\Lambda_{i}$, see Nil96 3.3]. The isomorphism takes $[f] \in J_{i} / J_{i-1}$ to the section $\widehat{f}$ defined by

$$
\widehat{f}(\pi)=\widehat{\pi}(f)=\int_{G} f(x) \pi(x) \mathrm{d} x \in \mathbb{B}\left(\mathcal{H}_{\pi}\right) \quad \text { for } \pi \in \Lambda_{i} .
$$

The dilation action on $J_{i} / J_{i-1}$ satisfies $\widehat{\sigma_{\lambda}(f)}(\pi)=\widehat{f}\left(\lambda^{-1} \cdot \pi\right)$ for all $\lambda>0$. Denote by $\alpha_{\lambda}(\widehat{f})$ the section given by $\alpha_{\lambda}(\widehat{f})(\pi)=\widehat{f}\left(\lambda^{-1} \cdot \pi\right)$. Let $\theta_{i}: \mathrm{C}_{0}\left(\Lambda_{i}\right) \hookrightarrow$ 
$Z \mathcal{M}\left(J_{i} / J_{i-1}\right)$ denote the non-degenerate homomorphism which is given by pointwise multiplication when $J_{i} / J_{i-1}$ is viewed as a continuous field. It satisfies the compatibility condition

$$
\alpha_{\lambda}\left(\theta_{i}(\phi) \widehat{f}\right)=\theta_{i}\left(\tau_{\lambda} \phi\right) \alpha_{\lambda}(\widehat{f}) \quad \text { for } \phi \in \mathrm{C}_{0}\left(\Lambda_{i}\right) \text { and }[f] \in J_{i} / J_{i-1},
$$

where $\tau$ denotes the $\mathbb{R}_{>0}$-action on $\mathrm{C}_{0}\left(\Lambda_{i}\right)$ given by $\tau_{\lambda}(\phi)(\pi)=\phi\left(\lambda^{-1} \cdot \pi\right)$. Therefore, $J_{i} / J_{i-1}$ is an $\mathbb{R}_{>0}-C_{0}\left(\Lambda_{i}\right)$-algebra. The dilation action on $\Lambda_{i}$ is free and proper by Proposition 3.35 By Proposition 2.28 $J_{i} / J_{i-1}$ is saturated with respect to the subset

$$
\mathcal{R}_{i}:=\overline{\theta_{i}\left(\mathrm{C}_{c}\left(\Lambda_{i}\right)\right)\left(J_{i} / J_{i-1}\right)} .
$$

It is the unique dense, complete, relatively continuous subset by Theorem 2.10 as $J_{i} / J_{i-1}$ is spectrally proper.

Using Corollary 2.22 and an inductive argument for the sequence in (48), we obtain as a consequence:

Corollary 10.2. For a homogeneous Lie group $G$ the $\mathbb{R}_{>0}-\mathrm{C}^{*}$-algebra $\left(J_{G}, \overline{\mathcal{R}_{G}}\right)$ is saturated for the dilation action of $\mathbb{R}_{>0}$. The generalized fixed point algebra $\operatorname{Fix}^{\mathbb{R}_{>0}}\left(J_{G}, \overline{\mathcal{R}_{G}}\right)$ is Morita-Rieffel equivalent to $\mathrm{C}_{\mathrm{r}}^{*}\left(\mathbb{R}_{>0}, J_{G}\right)$.

Recall that it was shown in Corollary 8.20 that for a graded Lie group $G$, $\operatorname{Fix}^{\mathbb{R}>0}\left(J_{G}, \overline{\mathcal{R}_{G}}\right)$ is the $\mathrm{C}^{*}$-algebra generated by kernels of type 0 . As an application of the above results, we give a different proof of the description of its spectrum obtained in [FFK17, 5.5].

Proposition 10.3. Let $G$ be a homogeneous Lie group. Then $\operatorname{Fix}^{\mathbb{R}>0}\left(J_{G}, \overline{\mathcal{R}_{G}}\right)$ is of type I. Furthermore, there is a homeomorphism

$$
\left.\left(\widehat{G} \backslash\left\{\pi_{\text {triv }}\right\}\right) / \mathbb{R}_{>0} \rightarrow \operatorname{Fix}^{\mathbb{R}>0} \widehat{\left(J_{G}\right.}, \overline{\mathcal{R}_{G}}\right)
$$

induced by $\pi \mapsto(\widehat{\pi})^{\sim}$ for $\pi \in \widehat{G} \backslash\left\{\pi_{\text {triv }}\right\}$.

Proof. The ideals in (48) yield short exact sequences of generalized fixed point algebras for $i=1, \ldots, m$ by Proposition 2.24

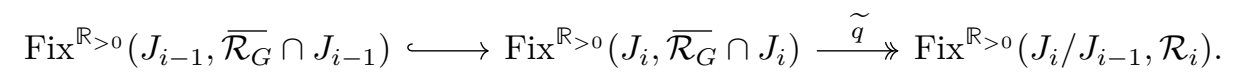

Each quotient $J_{i} / J_{i-1}$ is an $\mathbb{R}_{>0}-\mathrm{C}_{0}\left(\Lambda_{i}\right)$-algebra with a free and proper $\mathbb{R}_{>0}$-action on $\Lambda_{i}$ by Proposition 10.1 Therefore, the spectrum of $\operatorname{Fix}^{\mathbb{R}>0}\left(J_{i} / J_{i-1}, \mathcal{R}_{i}\right)$ is $\Lambda_{i} / \mathbb{R}_{>0}$ by Proposition 2.28 In particular, the spectrum is Hausdorff and, thus, $T_{0}$. $\operatorname{As~Fix~}^{\mathbb{R}>0}\left(J_{i} / J_{i-1}, \mathcal{R}_{i}\right)$ is separable, this implies that it is of type I by Dix77, 3.1.6, 9.1]. If an ideal $I$ of a $\mathrm{C}^{*}$-algebra $A$ and the quotient $A / I$ are of type $\mathrm{I}$, it follows that $A$ is of type I. Thus, one can use an inductive argument for the sequences above to show that $\operatorname{Fix}^{\mathbb{R}>0}\left(J_{G}, \overline{\mathcal{R}_{G}}\right)$ is of type I.

We proceed by showing the second claim. The spectrum of $J_{G}$ is given by

$$
\widehat{J_{G}}=\widehat{G} \backslash\left\{\pi_{\text {triv }}\right\}=\Lambda_{1} \cup \ldots \cup \Lambda_{m} .
$$

By Proposition $3.35, \mathbb{R}_{>0}$ acts freely on $\Lambda_{i}$ for all $i=1, \ldots, m$. Hence, the action of $\mathbb{R}_{>0}$ on the spectrum of $J_{G}$ is free. As $J_{G}$ and $\operatorname{Fix}^{\mathbb{R}_{>0}}\left(J_{G}, \overline{\mathcal{R}_{G}}\right)$ are of type I and separable, their spectra can be identified with their primitive ideal spaces. We show that there is a homeomorphism

$$
\psi: \operatorname{Prim}\left(J_{G}\right) / \mathbb{R}_{>0} \rightarrow \operatorname{Prim}\left(\operatorname{Fix}^{\mathbb{R}_{>0}}\left(J_{G}, \overline{\mathcal{R}_{G}}\right)\right)
$$

with $\psi([\operatorname{ker}(\pi)])=\operatorname{ker}(\tilde{\pi})$ for $\pi \in \widehat{J_{G}}$. By $\left.\mathbf{K M 2 0}, 6.3,6.4\right]$ there is a continuous, open and surjective quasi-orbit map

$$
\rho: \operatorname{Prim}\left(\mathrm{C}_{\mathrm{r}}^{*}\left(\mathbb{R}_{>0}, J_{G}\right)\right) \rightarrow \operatorname{Prim}\left(J_{G}\right) / \mathbb{R}_{>0} .
$$


As the action of the amenable group $\mathbb{R}_{>0}$ on $\operatorname{Prim}\left(J_{G}\right)$ is free, the quasi-orbit map is also injective by GR79 3.3]. Hence, it is a homeomorphism. In particular, $J_{G}$ separates ideals in $\mathrm{C}_{\mathrm{r}}^{*}\left(\mathbb{R}_{>0}, J_{G}\right)$. We describe now the inverse of $\rho$. First, there is a homeomorphism $\phi: \operatorname{Prim}\left(J_{G}\right) \mathbb{R}_{>0} \rightarrow \operatorname{Prime}\left(\mathbb{Q}^{\mathbb{R}}>0\left(J_{G}\right)\right)$ by $\mathbf{K M 2 0}$ 6.3], which is induced by mapping a primitive ideal $P$ of $J_{G}$ to the largest $\mathbb{R}_{>0}$-invariant ideal contained in $P$ :

$$
P \mapsto \bigcap_{\lambda \in \mathbb{R}_{>0}} \lambda \cdot P
$$

It follows from $\mathbf{K M 2 0} 2.11,6.1,6.3]$ that $\rho^{-1}=i \circ \phi$ with

$$
\begin{aligned}
i: \operatorname{Prime}\left(\mathbb{Q}^{\mathbb{R}>0}\left(J_{G}\right)\right) & \rightarrow \operatorname{Prim}\left(\mathrm{C}_{\mathrm{r}}^{*}\left(\mathbb{R}_{>0}, J_{G}\right)\right), \\
Q & \mapsto \mathrm{C}_{\mathrm{r}}^{*}\left(\mathbb{R}_{>0}, Q\right) .
\end{aligned}
$$

By Corollary 10.2 $\mathrm{C}_{\mathrm{r}}^{*}\left(\mathbb{R}_{>0}, J_{G}\right)$ is Morita-Rieffel equivalent to $\operatorname{Fix}^{\mathbb{R}>0}\left(J_{G}, \overline{\mathcal{R}_{G}}\right)$. Therefore, the Rieffel correspondence gives a homeomorphism

$$
r: \operatorname{Prim}\left(\mathrm{C}_{\mathrm{r}}^{*}\left(\mathbb{R}_{>0}, J_{G}\right)\right) \rightarrow \operatorname{Prim}\left(\operatorname{Fix}^{\mathbb{R}>0}\left(J_{G}, \overline{\mathcal{R}_{G}}\right)\right) .
$$

Together, we obtain a homeomorphism

$$
\psi:=r \circ \rho^{-1}: \operatorname{Prim}\left(J_{G}\right) / \mathbb{R}_{>0} \rightarrow \operatorname{Prim}\left(\operatorname{Fix}^{\mathbb{R}_{>0}}\left(J_{G}, \overline{\mathcal{R}_{G}}\right)\right) .
$$

It is left to show that $\psi([\operatorname{ker}(\pi)])=\operatorname{ker}(\widetilde{\pi})$ for $\pi \in \widehat{J_{G}}$. Let $Q=\phi([\operatorname{ker}(\pi)])$. Using that the action on $J_{G}$ is saturated, Lemma 2.21 implies

$$
\rho^{-1}([\operatorname{ker}(\pi)])=\mathrm{C}_{\mathrm{r}}^{*}\left(\mathbb{R}_{>0}, Q\right)=\mathrm{C}_{\mathrm{r}}^{*}\left(\mathbb{R}_{>0}, Q\right) \cap \mathrm{C}_{\mathrm{r}}^{*}\left(\mathbb{R}_{>0}, J_{G}\right)=J^{\mathbb{R}_{>0}}\left(Q, \overline{\mathcal{R}_{G}} \cap Q\right) .
$$

This ideal is mapped to $\operatorname{Fix}^{\mathbb{R}_{>0}}\left(Q, \overline{\mathcal{R}_{G}} \cap Q\right)$ under the Rieffel correspondence.

We show that $\operatorname{Fix}^{\mathbb{R}>0}\left(Q, \overline{\mathcal{R}}_{G} \cap Q\right)=\operatorname{ker}(\tilde{\pi})$. Let $a, b \in \overline{\mathcal{R}_{G}} \cap Q$. Then $\pi(a)=\pi(b)=0$ and, consequently, $\widetilde{\pi}(|a\rangle\rangle\langle\langle b|)=|\pi(a)\rangle\rangle\langle\langle\pi(b)|=0$. It follows that $\operatorname{Fix}^{\mathbb{R}>0}\left(Q, \overline{\mathcal{R}_{G}} \cap Q\right) \subseteq \operatorname{ker}(\widetilde{\pi})$.

Now let $T \in \operatorname{ker}(\tilde{\pi})$. As elements of the generalized fixed point algebra are invariant under the $\mathbb{R}_{>0}$-action, $(\lambda \cdot \pi)^{\sim}(T)=\widetilde{\pi}(T)=0$ holds for all $\lambda>0$. We use a similar argument as in the proof of Proposition 2.24 and show that $T^{*} T \in \operatorname{Fix}^{\mathbb{R}_{>0}}\left(Q, \overline{\mathcal{R}_{G}} \cap Q\right)$. For each $a \in \overline{\mathcal{R}_{G}}$, we obtain

$$
(\lambda \cdot \pi)\left(T^{*} a\right)=(\lambda \cdot \pi)^{\sim}\left(T^{*}\right)(\lambda \cdot \pi)(a)=0 \text { for all } \lambda>0 .
$$

It follows that $T^{*} a \in \overline{\mathcal{R}_{G}} \cap Q$. Now the same argument as in Proposition 2.24 shows that $T \in \operatorname{Fix}^{\mathbb{R}>0}\left(Q, \overline{\mathcal{R}_{G}} \cap Q\right)$.

\subsection{Filtered manifolds}

We deduce saturatedness for the respective ideals in the $\mathrm{C}^{*}$-algebras of the osculating groupoid and the tangent groupoid.

Proposition 10.4. Let $(M, H)$ be a filtered manifold. The $\mathrm{C}^{*}$-algebra of order 0 principal cosymbols $\operatorname{Fix}^{\mathbb{R}_{>0}}\left(\mathbb{J}_{0}, \overline{\mathcal{R}_{0}}\right)$ is Morita-Rieffel equivalent to $\mathrm{C}_{\mathrm{r}}^{*}\left(\mathbb{R}_{>0}, \mathbb{J}_{0}\right)$. The $\mathrm{C}^{*}$-algebra of order 0 pseudodifferential operators $\operatorname{Fix}^{\mathbb{R}>0}(\mathbb{J}, \overline{\mathcal{R}})$ is MoritaRieffel equivalent to $\mathrm{C}_{\mathrm{r}}^{*}\left(\mathbb{R}_{>0}, \mathbb{J}\right)$.

Proof. As all $\left(J_{x}, \overline{\mathcal{R}_{x}}\right)$ are saturated by Corollary $10.2,\left(\mathbb{J}_{0}, \overline{\mathcal{R}_{0}}\right)$ is saturated by Corollary 2.33 Therefore, the generalized fixed point algebra construction gives the Morita-Rieffel equivalence between $\operatorname{Fix}^{\mathbb{R}_{>0}}\left(\mathbb{J}_{0}, \overline{\mathcal{R}_{0}}\right)$ and $\mathrm{C}_{\mathrm{r}}^{*}\left(\mathbb{R}_{>0}, \mathbb{J}_{0}\right)$.

The second claim follows from Corollary 2.22 applied to the sequence in (31) if saturatedness for the ideal $\mathrm{C}_{0}\left(\mathbb{R}_{>0}\right) \otimes \mathbb{K}\left(L^{2} M\right)$ is shown. By Lemma 6.17 the $\mathbb{R}_{>0}$-action is given by $\tau \otimes 1$, where $\tau$ is induced by the action of $\mathbb{R}_{>0}$ on itself by multiplication. Then $\mathcal{R} \cap\left(\mathrm{C}_{0}\left(\mathbb{R}_{>0}\right) \otimes \mathbb{K}\left(L^{2} M\right)\right)$ is the unique dense, relatively continuous and complete subspace and $\tau$ is free and proper. Therefore, the action 
is saturated by Proposition 2.28. The Morita-Rieffel equivalence follows again from the generalized fixed point algebra construction. 


\section{CHAPTER 11}

\section{K-Theory and index theory}

Let $M$ be a compact, smooth manifold and let $E, F$ be smooth vector bundles over $M$. The Atiyah-Singer Index Theorem AS68 states that the analytical and topological index of an elliptic (pseudo)differential operator $P: \Gamma^{\infty}(E) \rightarrow \Gamma^{\infty}(F)$ coincide. The analytical index is the Fredholm index of $P$ when considered as a bounded operator between suitable Sobolev spaces.

Definition 11.1. A bounded operator $P: \mathcal{H}_{1} \rightarrow \mathcal{H}_{2}$ between two Hilbert spaces $\mathcal{H}_{1}, \mathcal{H}_{2}$ is Fredholm if its kernel and cokernel are finite-dimensional. In this case, its Fredholm index is defined as

$$
\operatorname{ind}(P)=\operatorname{dim}(\operatorname{ker} P)-\operatorname{dim}(\operatorname{coker} P) \in \mathbb{Z} .
$$

The properties of the classical pseudodifferential calculus on a compact manifold imply that elliptic operators are Fredholm. However, there are differential operators that are not elliptic but still admit a Fredholm index, see [BvE14, 2.3]. As a first step towards index theory, it is helpful to find a pseudodifferential calculus in which the considered operator is "elliptic" in a suitable sense.

Van Erp and Baum studied index theory for contact manifolds in $\mathbf{v E 1 0 a}$ vE10b BvE14. They solved the index problem for (pseudo)differential operators that are Heisenberg-elliptic. In [Moh20], Mohsen proved an index theorem for filtered manifolds.

In this section, we describe some consequences of the generalized fixed point algebra construction regarding K-theory and index theory. Let $(M, H)$ be a filtered manifold. If $M$ is compact, the $\mathrm{C}^{*}$-algebra $\operatorname{Fix}^{\mathbb{R}_{>0}}\left(\mathbb{J}_{0}, \overline{\mathcal{R}_{0}}\right)$ is unital by Lemma 8.12 We treat the generalized fixed point algebra extension

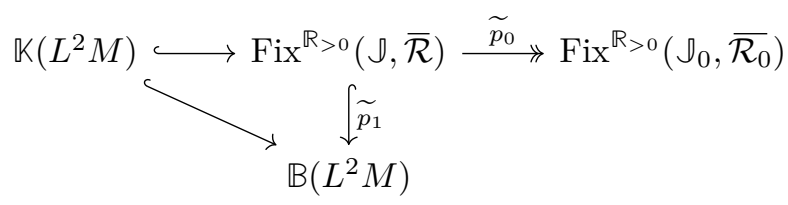

from (37) as an abstract pseudodifferential extension.

Definition 11.2. An operator $P \in \operatorname{Fix}^{\mathbb{R}>0}(\mathbb{J}, \overline{\mathcal{R}})$ is $\mathrm{C}^{*}-H$-elliptic if its principal symbol $\widetilde{p}_{0}(P)$ is invertible.

Since the sequence above is exact, $P$ is $\mathrm{C}^{*}-H$-elliptic if and only if there is a $Q \in \operatorname{Fix}^{\mathbb{R}>0}(\mathbb{J}, \overline{\mathcal{R}})$ such that $1-P Q, 1-Q P \in \mathbb{K}\left(L^{2} M\right)$. In particular, $P$ is Fredholm in this case by Atkinson's Theorem Atk51. As $\widetilde{p}_{0}(P)$ is invertible, it defines a class in $\mathrm{K}_{1}\left(\mathrm{Fix}^{\mathbb{R}_{>0}}\left(\mathbb{\mathbb { V }}_{0}, \overline{\mathcal{R}_{0}}\right)\right)$. The same arguments as in the unfiltered case yield the following lemma.

Lemma 11.3. The short exact sequence in 49 induces a 6-term exact sequence in K-theory. Let

$$
\partial: \mathrm{K}_{1}\left(\operatorname{Fix}^{\mathbb{R}>0}\left(\mathbb{J}_{0}, \overline{\mathcal{R}_{0}}\right)\right) \rightarrow \mathrm{K}_{0}\left(\mathbb{K}\left(L^{2} M\right)\right)
$$


denote the corresponding index map. For a $\mathrm{C}^{*}$-H-elliptic $P \in \operatorname{Fix}^{\mathbb{R}>0}(\mathbb{J}, \overline{\mathcal{R}})$ the Fredholm index of $P$ is given by $\partial\left(\left[\widetilde{p}_{0}(P)\right]\right.$ under the identification $\mathrm{K}_{0}(\mathbb{K}) \cong \mathbb{Z}$.

In particular, the Fredholm index just depends on the K-theory class of the principal symbol.

\section{1. $H$-Ellipticity and the Rockland condition}

The goal of this section is to understand $\mathrm{C}^{*}-H$-ellipticity better. Moreover, $\mathrm{C}^{*}$ - $H$-ellipticity is compared to $H$-ellipticity, which was defined by van Erp and Yuncken. We also discuss the relation to the Rockland condition.

Definition $11.4\left(\right.$ vEY17, 54]). An operator $P \in \Psi_{H}^{m}(M)$ on a compact filtered manifold $(M, H)$ is $H$-elliptic if its principal cosymbol $s_{H}^{m}(P)$ is invertible in $\mathcal{E}_{r}^{\prime}\left(T_{H} M\right) / \mathrm{C}_{c}^{\infty}\left(T_{H} M\right)$.

If $P \in \Psi_{H}^{m}(M)$ is $H$-elliptic, it admits a two-sided parametrix $Q \in \Psi_{H}^{-m}(M)$, that is, $P Q-1, Q P-1 \in \mathrm{C}_{c}^{\infty}(M \times M)$, see vEY17, 60]. If $P$ is an $H$-elliptic differential operator, this implies that $P$ is hypoelliptic [vEY17, 61].

For $m=0, P$ is $H$-elliptic if and only if $s_{H}^{0}(P)$ is invertible in $\Sigma_{H}^{0}(M)$. This follows from $\mathbf{v E Y 1 7}$, 55].

The $\mathrm{C}^{*}$-algebra of principal cosymbols $\operatorname{Fix}^{\mathbb{R}>0}\left(\mathbb{J}_{0}, \overline{\mathcal{R}_{0}}\right)$ is a continuous field of $\mathrm{C}^{*}$-algebras over $M$ with fibres $\operatorname{Fix}^{\mathbb{R}>0}\left(J_{x}, \overline{\mathcal{R}_{x}}\right)$ by Proposition 7.8 .

Lemma 11.5. Let $A$ be a unital $\mathrm{C}^{*}$-algebra. Suppose $A$ is a continuous field of $\mathrm{C}^{*}$-algebras over a compact Hausdorff space $X$ with fibre projections $q_{x}: A \rightarrow A_{x}$. Then $a \in A$ is invertible if and only if $a_{x}:=q_{x}(a)$ is invertible for all $x \in X$.

Proof. Clearly, invertibility of $a$ implies that $q_{x}(a)$ is invertible for all $x \in M$. Conversely, suppose that $q_{x}(a)$ is invertible at every point $x \in M$. Fix $x \in X$ and let $b_{x} \in A_{x}$ be an inverse of $a_{x}$. As $q_{x}$ is surjective, there is a $b \in A$ with $q_{x}(b)=b_{x}$. Let $c:=1-a b$. Because $q_{x}(c)=0$, one can find an open neighbourhood $U_{x}$ of $x$ such that $\left\|q_{y}(c)\right\| \leq 1 / 2$ for all $y \in U_{x}$ by continuity. By the von Neumann series $a b$ is locally invertible on $U_{x}$. Therefore, $b(a b)^{-1}$ is right inverse to $a$ on $U_{x}$. Using a continuous partition of unity which is subordinate to the open cover $U_{x}$ of $X$, one can glue together the local inverses to a global right inverse of $a$. Similarly, one can construct a left inverse of $a$. It follows that $a$ is invertible.

Therefore, $\mathrm{C}^{*}-H$-ellipticity is a pointwise condition.

Corollary 11.6. Let $(M, H)$ be a compact filtered manifold. A principal cosymbol $u \in \operatorname{Fix}^{\mathbb{R}_{>0}}\left(\mathbb{J}_{0}, \overline{\mathcal{R}_{0}}\right)$ is $\mathrm{C}^{*}-H$-elliptic if and only if $u_{x} \in \operatorname{Fix}^{\mathbb{R}_{>0}}\left(J_{x}, \overline{\mathcal{R}_{x}}\right)$ is invertible for all $x \in M$.

Consider now the Rockland condition as described in Pon08, Sec. 3.3.2] and DH17. Sec. 3.4]. It generalizes the Rockland condition for differential operators in Definition 9.21. Let $G$ be a graded Lie group of homogeneous dimension $Q$ and $m \in \mathbb{Z}$. Let $u \in \mathcal{E}^{\prime}(G) / \mathrm{C}_{c}^{\infty}(G)$ be $m$-homogeneous. It was shown in DH17, 3.8] that the class $u \in \mathcal{E}^{\prime}(G) / \mathrm{C}_{c}^{\infty}(G)$ can be uniquely represented by a kernel $a$ of type $-m$ if $-m-Q \notin \mathbb{N}_{0}$. For $m=0$ this is Lemma 9.11. If $-m-Q \in \mathbb{N}_{0}$, it can be represented by $a=k+p \log (\|x\|)$ with $k \in \mathcal{K}^{-m}(G)$ and a $(-m-Q)$-homogeneous polynomial $p$. This representation is not necessarily unique. However, the map $\mathcal{S}_{0}(G) \rightarrow \mathcal{S}_{0}(G)$ given by $f \mapsto a * f$ does not depend on the chosen representative $a$ of $u$ (see DH17, Sec. 3.4]). Here, $\mathcal{S}_{0}(G)$ is as in Definition 8.7

For a unitary, irreducible representation $\pi: G \rightarrow \mathcal{U}\left(\mathcal{H}_{\pi}\right)$, let $\mathcal{H}_{\pi}^{0}$ be spanned by $\widehat{\pi}(f) v$ for $f \in \mathcal{S}_{0}(G)$ and $v \in \mathcal{H}_{\pi}$. The operator $\pi(u)$ defined on $\mathcal{H}_{\pi}^{0}$ by

$$
\pi(u)(\widehat{\pi}(f) v):=\widehat{\pi}(a * f) v \quad \text { for } f \in \mathcal{S}_{0}(G), v \in \mathcal{H}_{\pi},
$$


is closable. Denote its closure by $\overline{\pi(u)}$.

Definition 11.7. Let $G$ be a graded Lie group and let $u \in \mathcal{E}^{\prime}(G) / \mathrm{C}_{c}^{\infty}(G)$ be homogeneous. Then $u$ satisfies the Rockland condition if $\overline{\pi(u)}$ is injective on $\mathcal{H}_{\pi}^{\infty}$ for all $\pi \in \widehat{G} \backslash\left\{\pi_{\text {triv }}\right\}$. Here, $\mathcal{H}_{\pi}^{\infty}$ denotes the space of smooth vectors.

Example 11.8. Recall that for a usual compact manifold of dimension $n$ the osculating groups are isomorphic to $\mathbb{R}^{n}$. Let $P$ be a pseudodifferential operator on $M$ with model operators $\Sigma(P)_{x}$ for $x \in M$. Then $\Sigma(P)_{x}$ satisfies the Rockland condition if and only if $\widehat{\Sigma(P)_{x}}(\xi) \neq 0$ for all $\xi \neq 0$. Therefore, $P$ is elliptic if and only if $\Sigma(P)_{x}$ satisfies the Rockland condition for all $x \in M$.

For a homogeneous Lie group $G, \operatorname{Fix}^{\mathbb{R}>0}\left(J_{G}, \overline{\mathcal{R}_{G}}\right)$ is the $\mathrm{C}^{*}$-algebra of kernels of type 0 by Corollary 8.20 . The spectrum of $\operatorname{Fix}^{\mathbb{R}_{>0}}\left(J_{G}, \overline{\mathcal{R}_{G}}\right)$ is $\left(\widehat{G} \backslash\left\{\pi_{\text {triv }}\right\}\right) / \mathbb{R}_{>0}$ by Proposition 10.3 This allows to describe invertibility in $\operatorname{Fix}^{\mathbb{R}_{>0}}\left(J_{G}, \overline{\mathcal{R}_{G}}\right)$ in terms of the representations of $G$.

Definition 11.9. An element $u \in \operatorname{Fix}^{\mathbb{R}>0}\left(J_{G}, \overline{\mathcal{R}_{G}}\right)$ satisfies the $\mathrm{C}^{*}$-Rockland condition if $\tilde{\pi}(u)$ is invertible for all $\pi \in \widehat{G} \backslash\left\{\pi_{\text {triv }}\right\}$.

Proposition 11.10. Let $u \in \operatorname{Fix}^{\mathbb{R}_{>0}}\left(J_{G}, \overline{\mathcal{R}_{G}}\right)$. Then $u$ is invertible if and only if $u$ satisfies the $\mathrm{C}^{*}$-Rockland condition.

Proof. Suppose $u \in \operatorname{Fix}^{\mathbb{R}>0}\left(J_{G}, \overline{\mathcal{R}_{G}}\right)$ is invertible. As $\tilde{\pi}$ for $\pi \neq \pi_{\text {triv }}$ is an irreducible representation of the unital $\mathrm{C}^{*}$-algebra $\operatorname{Fix}^{\mathbb{R}_{>0}}\left(J_{G}, \overline{\mathcal{R}_{G}}\right)$, it follows that $\tilde{\pi}(u)$ is invertible.

Now assume that $u$ satisfies the $\mathrm{C}^{*}$-Rockland condition. As $\operatorname{Fix}^{\mathbb{R}>0}\left(J_{G}, \overline{\mathcal{R}_{G}}\right)$ is a $C^{*}$-subalgebra of the group von Neumann algebra of $G$, it suffices to show invertibility in this larger $\mathrm{C}^{*}$-algebra. The group von Neumann algebra is isomorphic to $L^{\infty}\left(\widehat{G}, \mathbb{B}\left(\mathcal{H}_{\pi}\right)\right)$ via Fourier transform. As $u$ is invariant under $\mathbb{R}_{>0}$ and $\left\{\pi_{\text {triv }}\right\}$ is a null set, we show that the essential infimum of the function

$$
\left(\widehat{G} \backslash\left\{\pi_{\text {triv }}\right\}\right) / \mathbb{R}_{>0} \rightarrow[0, \infty] \quad \pi \mapsto\|\widetilde{\pi}(u)\|,
$$

is strictly greater than zero. The domain is the spectrum of $\operatorname{Fix}^{\mathbb{R}>0}\left(J_{G}, \overline{\mathcal{R}_{G}}\right)$, thus the function is lower semi-continuous, see $\mathbf{R W 9 8}$, A.30]. As $\left(\widehat{G} \backslash\left\{\pi_{\text {triv }}\right\}\right) / \mathbb{R}_{>0}$ is compact as the spectrum of a unital $\mathrm{C}^{*}$-algebra, the function attains its minimum. It must be strictly greater than zero since $u$ satisfies the $\mathrm{C}^{*}$-Rockland condition.

Now, one deduces that $P \in \Psi_{H}^{0}(M)$ is $\mathrm{C}^{*}-H$-elliptic if and only if it is $H$-elliptic.

Proposition 11.11. Let $(M, H)$ be a compact filtered manifold. For $P \in \Psi_{H}^{0}(M)$ the following are equivalent:

(i) $P$ is $\mathrm{C}^{*}-H$-elliptic,

(ii) $s_{H}^{0}(P)_{x}$ satisfies the $\mathrm{C}^{*}$-Rockland condition for all $x \in M$,

(iii) $s_{H}^{0}(P)_{x}$ and $s_{H}^{0}(P)_{x}^{*}$ satisfy the Rockland condition for all $x \in M$,

(iv) $P$ is $H$-elliptic.

Proof. The equivalence of (i) and (ii) follows from Corollary 11.6 and Proposition 11.10 By the results of Głowacki in Gło91, 4.3 and 4.9], (ii) and (iii) are equivalent for all $x \in M$. The arguments in [DH17, 3.11, 3.12], which follow CGGP92 and Pon08, show that (iii) and (iv) are equivalent.

Remark 11.12. The Rockland condition is also defined for operators acting between vector bundles $E, F$ over $M$ (see Pon08, Sec. 3.3.2] or [DH17, Sec. 3.4]). In this case, the model operators map $\mathcal{S}_{0}\left(G(x), E_{x}\right) \rightarrow \mathcal{S}_{0}\left(G(x), F_{x}\right)$. It is shown in DH17, 3.11, 3.12] that $H$-ellipticity is again equivalent to satisfying the Rockland condition at all points. 


\subsection{Deformation to the step 1 case}

For a filtered manifold $(M, H)$, consider the restriction of the short exact sequence in 24 to $[0,1]$

$$
\mathrm{C}_{0}((0,1]) \otimes \mathbb{K}\left(L^{2} M\right) \longleftrightarrow \mathrm{C}^{*}\left(\left.\mathbb{T}_{H} M\right|_{[0,1]}\right) \stackrel{\mathrm{ev}_{0}^{H}}{\longrightarrow} \mathrm{C}^{*}\left(T_{H} M\right) .
$$

The $\mathrm{C}^{*}$-algebra on the left is contractible and $\mathrm{C}^{*}\left(T_{H} M\right)$ is nuclear as a bundle of nilpotent Lie groups. Therefore, the class $\left[\mathrm{ev}_{0}^{H}\right] \in \mathrm{KK}\left(\mathrm{C}^{*}\left(\left.\mathbb{T}_{H} M\right|_{[0,1]}\right), \mathrm{C}^{*}\left(T_{H} M\right)\right)$ is invertible. As described in [DL10] one can define a deformation element

$$
\left[\mathrm{ev}_{0}^{H}\right]^{-1} \otimes\left[\mathrm{ev}_{1}^{H}\right] \in \mathrm{KK}\left(\mathrm{C}^{*}\left(T_{H} M\right), \mathbb{K}\right)
$$

associated to the short exact sequence above. Likewise, there is a deformation element $\left[\mathrm{ev}_{0}\right]^{-1} \otimes\left[\mathrm{ev}_{1}\right] \in \mathrm{KK}\left(\mathrm{C}_{0}\left(T^{*} M\right), \mathbb{K}\right)$ for the short exact sequence of Connes' tangent groupoid

$$
\mathrm{C}_{0}((0,1]) \otimes \mathbb{K}\left(L^{2} M\right) \longleftrightarrow \mathrm{C}^{*}\left(\left.\mathbb{T} M\right|_{[0,1]}\right) \stackrel{\mathrm{ev}_{0}}{\longrightarrow} \mathrm{C}_{0}\left(T^{*} M\right) .
$$

Connes showed that this class is the analytical index (see Con94).

Using the adiabatic groupoid of $\mathbb{T}_{H} M$ one can relate these deformation classes in $\mathrm{KK}\left(\mathrm{C}^{*}\left(T_{H} M\right), \mathbb{K}\right)$ and $\mathrm{KK}\left(\mathrm{C}_{0}\left(T^{*} M\right), \mathbb{K}\right)$. This construction was carried out in vE10a for contact manifolds, see also Moh18 Moh20 for the filtered manifold case. In the following, we recall the argument.

The Lie algebroid $\mathbb{t}_{H} M$ of $\mathbb{T}_{H} M$ is described in $\mathbf{v E Y 1 7}$. Denote by $\rho: \mathbb{t}_{H} M \rightarrow$ $T(M \times[0,1])$ its anchor map and let

$$
[\cdot, \cdot]: \Gamma^{\infty}\left(\mathbb{E}_{H} M\right) \times \Gamma^{\infty}\left(\mathbb{E}_{H} M\right) \rightarrow \Gamma^{\infty}\left(\mathbb{E}_{H} M\right)
$$

be the bracket. Let $\mathbb{T}_{H} M^{a}$ be the adiabatic groupoid of $\mathbb{T}_{H} M$. It is easier to describe it in terms of its Lie algebroid (see DS14, 2.1]). It is the vector bundle $\mathbb{t}_{H} M \times \mathbb{R}$ over $M \times \mathbb{R} \times \mathbb{R}$ with anchor

$$
\begin{aligned}
\rho_{a}: \mathbb{E}_{H} M \times \mathbb{R} & \rightarrow T(M \times \mathbb{R}) \times T \mathbb{R} \\
\rho_{a}(x, t, U, s) & =(\rho(x, t, s U), s, 0)
\end{aligned}
$$

for $x \in M, t, s \in \mathbb{R}$ and $U \in \mathbb{E}_{H} M_{(x, t)}$. The bracket is defined by

$$
[X, Y]_{a}(x, t, s)=s[X, Y](x, t) \quad \text { for } X, Y \in \Gamma^{\infty}\left(\mathbb{E}_{H} M \times \mathbb{R}\right) .
$$

The resulting Lie groupoid can be viewed as a continuous field of groupoids over each copy of $\mathbb{R}$ and over $\mathbb{R}^{2}$. The fibre over $s=1$ is the tangent groupoid $T_{H} M$ of the filtered manifold.

For $s=0$, the anchor and bracket are zero. One obtains a bundle of Abelian groups. It is isomorphic to $T M \times[0,1]$ via a splitting as defined in $\mathbf{v E Y 1 7}, 9]$. A splitting is a vector bundle isomorphism $\mathfrak{t}_{H} M \rightarrow T M$, which restricts on $H^{\jmath} / H^{j-1}$ to a right inverse of $H^{j} \rightarrow H^{j} / H^{j-1}$ for $j=1, \ldots, r$.

For $t=0$ the anchor is zero. Therefore, all fibres over $(0, s)$ for $s \in \mathbb{R}$ are bundles of nilpotent groups. The bundle of osculating groups $T_{H} M$ at $s=1$ is deformed into a bundle of Abelian groups at $s=0$. The latter can be identified with $T M$ using the splitting above. Denote the subgroupoid at $t=0$ by $\mathcal{G}$.

Note that the fibre at $(1,1)$ is the pair groupoid of $M$. Its adiabatic groupoid is Connes' tangent groupoid $\mathbb{T} M$. It is the fibre at $t=1$ of $\mathbb{T}_{H} M^{a}$.

Therefore, all edges of $[0,1]^{2}$ can be understood as deformation groupoids and one can associate corresponding deformation classes in the respective KK-groups. 
Denote the restriction to the edges by $r_{t=1}, r_{t=0}, r_{s=1}, r_{s=0}$. The following diagram commutes

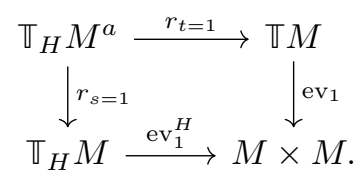

Therefore, the following KK-classes coincide:

$$
\left[r_{t=1}\right] \otimes\left[\mathrm{ev}_{1}\right]=\left[r_{s=1}\right] \otimes\left[\mathrm{ev}_{1}^{H}\right] .
$$

Denote by $b_{0}$ and $b_{1}$ the restrictions to $t=0$ and $t=1$ on the trivial bundle at $s=0$. The deformation class $\left[b_{0}\right]^{-1} \otimes\left[b_{1}\right]$ is the identity. It follows that

$$
\left[r_{t=1}\right] \otimes\left[\mathrm{ev}_{0}\right]=\left[r_{t=0}\right] \otimes\left[b_{1}\right]=\left[r_{t=0}\right] \otimes\left[b_{0}\right] .
$$

Denote by $c_{0}$ and $c_{1}$ the respective restrictions on $\mathcal{G}$. We obtain

$$
\left[r_{s=1}\right] \otimes\left[\mathrm{ev}_{0}^{H}\right]=\left[r_{t=0}\right] \otimes\left[c_{1}\right]=\left(\left[r_{t=0}\right] \otimes\left[c_{0}\right]\right) \otimes\left(\left[c_{0}\right]^{-1} \otimes\left[c_{1}\right]\right) .
$$

Let $r_{00}$ be the restriction to $t=0, s=0$. One can show that it induces a KK-equivalence as in $\mathbf{v E 1 0 a} 21]$. This is done by writing it as a composition of the restriction to the union of $t=0$ and $s=0$ and further restriction to $t=s=0$. Both maps have contractible kernels. Using (51) and (52), one obtains

$$
\begin{aligned}
& {\left[r_{00}\right]^{-1} \otimes\left[r_{t=1}\right]=\left[\mathrm{ev}_{0}\right]^{-1},} \\
& {\left[r_{00}\right]^{-1} \otimes\left[r_{s=1}\right]=\left(\left[c_{0}\right]^{-1} \otimes\left[c_{1}\right]\right) \otimes\left[\mathrm{ev}_{0}^{H}\right]^{-1} .}
\end{aligned}
$$

Inserting this into $(50)$ shows that the deformation classes for $\mathbb{\mathbb { T }}_{H} M$ and $\mathbb{\mathbb { T }} M$ are related by

$$
\left[\mathrm{ev}_{0}\right]^{-1} \otimes\left[\mathrm{ev}_{1}\right]=\left(\left[c_{0}\right]^{-1} \otimes\left[c_{1}\right]\right) \otimes\left(\left[\mathrm{ev}_{0}^{H}\right]^{-1} \otimes\left[\mathrm{ev}_{1}^{H}\right]\right)
$$

The class $\Psi:=\left(\left[c_{0}\right]^{-1} \otimes\left[c_{1}\right]\right) \in \mathrm{KK}\left(\mathrm{C}_{0}\left(T^{*} M\right), \mathrm{C}^{*}\left(T_{H} M\right)\right)$ is a KK-equivalence. This is a well-known consequence of the Connes-Thom isomorphism, see Con81. Corollary 7] and [Nis03. Corollary 1] for the bundle version. We show that the KK-equivalence $\Psi$ restricts to the ideals used in the generalized fixed point algebra construction.

Lemma 11.13. The $\mathrm{KK}$-equivalence $\Psi \in \mathrm{KK}\left(\mathrm{C}_{0}\left(T^{*} M\right), \mathrm{C}^{*}\left(T_{H} M\right)\right)$ restricts to a KK-equivalence $\Psi \mid \in \mathrm{KK}\left(\mathrm{C}_{0}\left(T^{*} M \backslash(M \times 0)\right), \mathbb{J}_{0}\right)$.

Proof. Define the ideal $\rrbracket_{\mathcal{G}} \subset \mathrm{C}^{*}(\mathcal{G})$ that consists of all sections $\left(a_{s}\right) \in \mathrm{C}^{*}(\mathcal{G})$ such that all $a_{s, x}$ for $s \in[0,1]$ and $x \in M$ lie in the kernel of the trivial representation of the nilpotent Lie group over $(s, x)$. The trivial representations induce a commuting diagram

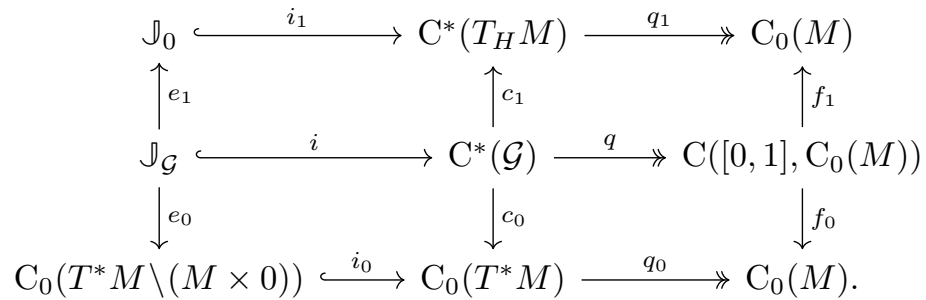

As $\operatorname{ker}\left(e_{0}\right)$ is contractible, one can build the deformation class $\Psi \mid:=\left(\left[e_{0}\right]^{-1} \circ\left[e_{1}\right]\right) \in$ $\mathrm{KK}\left(\mathrm{C}_{0}\left(T^{*} M \backslash(M \times 0)\right), \mathbb{J}_{0}\right)$. Similarly, there is a class $\alpha:=\left(\left[f_{0}\right]^{-1} \circ\left[f_{1}\right]\right) \in$ 
$\mathrm{KK}\left(\mathrm{C}_{0}(M), \mathrm{C}_{0}(M)\right)$. Because (53) commutes, there is a commuting diagram in $\mathrm{KK}$ :

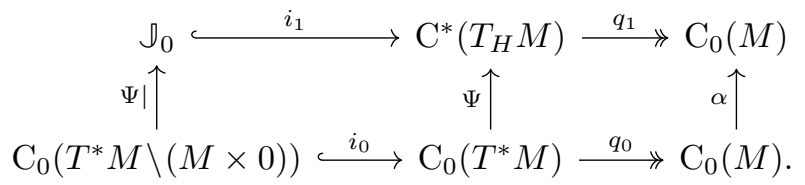

The KK-classes in the middle and on the right are KK-equivalences. The long exact sequences in KK-theory and the Five Lemma yield that

$$
\begin{aligned}
\left\llcorner\otimes \Psi \mid: \mathrm{KK}\left(A, \mathrm{C}_{0}\left(T^{*} M \backslash(M \times 0)\right)\right)\right. & \rightarrow \mathrm{KK}\left(A, \mathbb{J}_{0}\right) \\
\Psi \mid \otimes{ }_{-}: \mathrm{KK}\left(\mathbb{J}_{0}, B\right) & \rightarrow \mathrm{KK}\left(\mathrm{C}_{0}\left(T^{*} M \backslash(M \times 0)\right), B\right)
\end{aligned}
$$

are isomorphisms for all separable, nuclear $\mathrm{C}^{*}$-algebras $A, B$. Taking $A=\mathbb{I}_{0}$ and $B=\mathrm{C}_{0}\left(T^{*} M \backslash(M \times 0)\right)$, one obtains a class in $\mathrm{KK}\left(\mathbb{J}_{0}, \mathrm{C}_{0}\left(T^{*} M \backslash(M \times 0)\right)\right.$ that is the KK-inverse of $\Psi \mid$.

As a consequence, the $\mathrm{C}^{*}$-algebra of principal cosymbols of order 0 has the same K-theory as its unfiltered counterpart.

TheOREM 11.14. Let $(M, H)$ be a filtered manifold. The $\mathrm{C}^{*}$-algebra of principal cosymbols $\operatorname{Fix}^{\mathbb{R}_{>0}}\left(\mathbb{J}_{0}, \overline{\mathcal{R}_{0}}\right)$ and $\mathrm{C}_{0}\left(S^{*} M\right)$ are $\mathrm{KK}$-equivalent.

Proof. It was shown in Proposition 10.4 that $\operatorname{Fix}^{\mathbb{R}>0}\left(\mathbb{J}_{0}, \overline{\mathcal{R}_{0}}\right)$ is Morita-Rieffel equivalent to $\mathrm{C}_{\mathrm{r}}^{*}\left(\mathbb{R}_{>0}, \mathbb{J}_{0}\right)$. Therefore, they are KK-equivalent. As $\left(\mathbb{R}_{>0}, \cdot\right) \cong$ $(\mathbb{R},+)$ and by the Connes-Thom isomorphism, $\mathrm{C}_{\mathrm{r}}^{*}\left(\mathbb{R}_{>0}, \mathbb{J}_{0}\right)$ is KK-equivalent to $\mathrm{C}_{0}(\mathbb{R}) \otimes \mathbb{J}_{0}$. This $\mathrm{C}^{*}$-algebra is KK-equivalent to $\mathrm{C}_{0}(\mathbb{R}) \otimes \mathrm{C}_{0}\left(T^{*} M \backslash(M \times 0)\right)$ by Lemma 11.13 The converse argument, applied to the step 1 filtration case, yields that $\mathrm{C}_{0}(\mathbb{R}) \otimes \mathrm{C}_{0}\left(T^{*} M \backslash(M \times 0)\right)$ is KK-equivalent to $\mathrm{C}_{0}\left(S^{*} M\right)$.

\subsection{Connections to index theory}

In this section, let $(M, H)$ be a compact filtered manifold. Following the explanation in BvE14, 5.3] (see also Con94, §II.9. $\alpha]$ ), one can attach to an $H$-elliptic $H$-pseudodifferential operator $P$ of order $m$ a class in $\mathrm{K}_{0}\left(\mathrm{C}^{*}\left(T_{H} M\right)\right)$. Let $\mathbb{P}$ be a lift of $P$ to $\Downarrow_{H}^{m}(M)$. By definition, the equivalence class $\left[\mathbb{P}_{0}\right] \in \mathcal{E}_{r}^{\prime}\left(T_{H} M\right) / \mathrm{C}_{c}^{\infty}\left(T_{H} M\right)$ is invertible. So there is a $\mathbb{Q}_{0} \in \mathcal{E}_{r}^{\prime}\left(T_{H} M\right)$ with $S_{0}:=1-\mathbb{Q}_{0} * \mathbb{P}_{0} \in \mathrm{C}_{c}^{\infty}\left(T_{H} M\right)$ and $S_{1}:=1-\mathbb{P}_{0} * \mathbb{Q}_{0} \in \mathrm{C}_{c}^{\infty}\left(T_{H} M\right)$.

Let $\sigma_{H}(P):=[e]-\left[e_{0}\right] \in \mathrm{K}_{0}\left(\mathrm{C}^{*}\left(T_{H} M\right)\right)$ be the formal difference of idempotents

$$
e=\left(\begin{array}{cc}
1-S_{1}^{2} & \mathbb{P}_{0} * S_{0} \\
S_{0} * \mathbb{Q}_{0} *\left(1+S_{1}\right) & S_{0}^{2}
\end{array}\right) \quad \text { and } \quad e_{0}=\left(\begin{array}{cc}
1 & 0 \\
0 & 0
\end{array}\right) .
$$

The same construction works for operators that act on a vector bundles over $M$.

Lemma 11.15. Let $(M, H)$ be a compact filtered manifold. Consider the short exact sequence from 44 given by

$$
\mathrm{C}^{*}\left(T_{H} M\right) \longrightarrow \mathrm{C}^{*}\left(\operatorname{Ess}_{H}^{0}(M)\right) \longrightarrow \mathrm{C}^{*}\left(\Sigma_{H}^{0}(M)\right) .
$$

For an $H$-elliptic $P \in \Psi_{H}^{0}(M)$ the class $\sigma_{H}(P) \in \mathrm{K}_{0}\left(\mathrm{C}^{*}\left(T_{H} M\right)\right)$ above is the image of $\left[s_{H}^{0}(P)\right] \in \mathrm{K}_{1}\left(\mathrm{C}^{*}\left(\Sigma_{H}^{0}(M)\right)\right)$ under the boundary map in $\mathrm{K}$-theory of (54).

Proof. Let $\mathbb{Q}_{0} \in \mathcal{E}_{r}^{\prime}\left(T_{H} M\right)$ satisfy $1-\mathbb{Q}_{0} * \mathbb{P}_{0} \in \mathrm{C}_{c}^{\infty}\left(T_{H} M\right)$ and $1-\mathbb{P}_{0} * \mathbb{Q}_{0} \in$ $\mathrm{C}_{c}^{\infty}\left(T_{H} M\right)$ as above. By $\left.\mathbf{v E Y 1 9}, 55\right] \mathbb{Q}_{0}$ is contained in $\operatorname{Ess}_{H}^{0}(M)$. Hence, $\mathbb{P}_{0}$ and $Q_{0}$ are lifts of $\left[\mathbb{P}_{0}\right]$ and $\left[\mathbb{P}_{0}\right]^{-1}$ in $\operatorname{Ess}_{H}^{0}(M)$. Computing the image of $\left[s_{H}^{0}(P)\right]$ under the index map as in [CMR07, 1.46] gives exactly the class above. 
Up to inverting the Connes-Thom isomorphism, we prove an index theorem for $H$-elliptic pseudodifferential operators of order zero. For contact manifolds, this is vE10b Prop. 12] in the scalar-valued case or [BvE14, Thm. 5.4.1] for operators acting on vector bundles. Mohsen recently proved an index theorem for filtered manifolds in Moh20. His construction involves a "larger" bundle of graded Lie groups over $M$ to obtain an index theorem that does not contain the Connes-Thom isomorphism anymore.

THEOREM 11.16. Let $(M, H)$ be a compact filtered manifold and let $P$ be an order zero $H$-elliptic $H$-pseudodifferential operator acting on vector bundles $E, F$ over $M$. Then

$$
\operatorname{ind}(P)=\operatorname{ind}_{t}\left(\Psi^{-1}\left(\sigma_{H}(P)\right)\right)
$$

holds. Here, $\Psi: \mathrm{K}^{0}\left(T^{*} M\right) \rightarrow \mathrm{K}_{0}\left(\mathrm{C}^{*}\left(T_{H} M\right)\right)$ denotes the Connes-Thom isomorphism and $\operatorname{ind}_{t}: \mathrm{K}^{0}\left(T^{*} M\right) \rightarrow \mathbb{Z}$ is the topological index.

Proof. Choose hermitean metrics on $E$ and $F$. Using polar decomposition and that the Fredholm index is invariant under homotopies, we can assume without loss of generality that the principal cosymbol of $P$ is unitary.

We follow the arguments in $\mathbf{v E 1 0 a} \mathbf{v E 1 0 b}$ BvE14 closely. Let $\mathbb{E}:=E \times[0,1]$ and $\mathbb{E}:=F \times[0,1]$ denote the vector bundles over the unit space $M \times[0,1]$ of $\left.\mathbb{T}_{H} M\right|_{[0,1]}$ and extend $P$ to $\mathbb{P} \in \Downarrow_{H}^{0}(M, \mathbb{E}, \mathbb{F})$.

As in BvE14 construct a class $[\mathbb{D}] \in \mathrm{KK}\left(\mathrm{C}(M), \mathrm{C}^{*}\left(\left.\mathbb{T}_{H} M\right|_{[0,1]}\right)\right)$ from $\mathbb{P}$ as follows. Define the $\mathbb{Z}_{2}$-graded right Hilbert $\mathrm{C}^{*}\left(\left.\mathbb{T}_{H} M\right|_{[0,1]}\right)$-module

$$
\mathcal{E}=\Gamma_{0}(\mathbb{E}) \otimes_{\mathrm{C}(M \times[0,1])} \mathrm{C}^{*}\left(\left.\mathbb{T}_{H} M\right|_{[0,1]}\right) \oplus \Gamma_{0}(\mathbb{F}) \otimes_{\mathrm{C}(M \times[0,1])} \mathrm{C}^{*}\left(\left.\mathbb{T}_{H} M\right|_{[0,1]}\right)
$$

and let

$$
\mathbb{D}=\left(\begin{array}{cc}
0 & \mathbb{P}^{*} \\
\mathbb{P} & 0
\end{array}\right) \in \mathrm{C}^{*}\left(\mathbb{\Psi}_{H}^{0}(M)\right) \otimes \operatorname{End}(\mathbb{E} \oplus \mathbb{F}) .
$$

Note that elements of $\mathrm{C}^{*}\left(\Psi_{H}^{0}(M)\right)$ act as multipliers on $\mathrm{C}^{*}\left(\left.\mathbb{T}_{H} M\right|_{[0,1]}\right)$. By $\mathbf{v E Y 1 9}$ $25]$ there is a homomorphism $\mathrm{C}(M) \rightarrow \mathrm{C}^{*}\left(\Downarrow_{H}^{0}(M)\right), f \mapsto \mathbb{E}$, where $\mathbb{V}_{t}$ is the multiplication operator $M_{f}$ on $L^{2}(M)$ for $t>0$ and $\mathbb{E}_{0}$ is the fibred distribution given by $\left(f(x) \delta_{x}\right)_{x \in M}$. Therefore, there is a diagonal representation $\phi: \mathrm{C}(M) \rightarrow \mathcal{L}(\mathcal{E})$. Moreover, $\mathbb{D}$ acts as an odd operator on $\mathcal{E}$. We verify that $\phi(f)\left(\mathbb{D}^{2}-1\right)$ and $[\phi(f), \mathbb{D}]$ lie in $\mathcal{K}(\mathcal{E})$ for all $f \in \mathrm{C}(M)$. Compute

$$
\phi(f)\left(\mathbb{D}_{t}^{2}-1\right)=\phi(f)\left(\begin{array}{cc}
\mathbb{P}_{t}^{*} \mathbb{P}_{t}-1 & 0 \\
0 & \mathbb{P}_{t}^{*} \mathbb{P}_{t}-1
\end{array}\right) .
$$

At $t=0$, this defines a matrix over $\mathrm{C}^{*}\left(T_{H} M\right)$. Then the claim follows from Lemma 9.19 For $[\phi(f), \mathbb{D}]$, note that this vanishes at $t=0$ as functions in $\mathrm{C}(M)$ define central multipliers of $\mathrm{C}^{*}\left(T_{H} M\right)$. Therefore, Lemma 9.19 applies, too. Note that one can restrict $(\mathcal{E}, \phi, \mathbb{D})$ to $t \geq 0$ and denote the restricted classes by $\left[\mathbb{D}_{t}\right]$. The long exact sequence in KK-theory implies that

$$
\mathrm{ev}_{0}^{H}: \mathrm{KK}\left(\mathrm{C}(M), \mathrm{C}^{*}\left(\left.\mathbb{T}_{H} M\right|_{[0,1]}\right)\right) \rightarrow \mathrm{KK}\left(\mathrm{C}(M), \mathrm{C}^{*}\left(T_{H} M\right)\right)
$$

is invertible. Similar to before, one obtains a map

$$
\mathrm{ev}_{1}^{H} \circ\left(\mathrm{ev}_{0}^{H}\right)^{-1}: \mathrm{KK}\left(\mathrm{C}(M), \mathrm{C}^{*}\left(T_{H} M\right)\right) \rightarrow \mathrm{KK}(\mathrm{C}(M), \mathbb{C}) .
$$

It satisfies $\mathrm{ev}_{1}^{H} \circ\left(\mathrm{ev}_{0}^{H}\right)^{-1}\left(\left[\mathbb{D}_{0}\right]\right)=\left[\mathbb{D}_{1}\right]$. Let $[u] \in \mathrm{KK}(\mathbb{C}, \mathrm{C}(M))$ be the class induced by the unital embedding $\mathbb{C} \rightarrow \mathrm{C}(M)$. It is well-known that $[u] \otimes\left[\mathbb{D}_{1}\right] \in \mathrm{KK}(\mathbb{C}, \mathbb{C}) \cong$ $\mathbb{Z}$ is the class representing the Fredholm index of $P$ (see [CMR07, (12.7)]).

Similarly, there is a corresponding map for Connes' tangent groupoid

$$
\mathrm{ev}_{1} \circ\left(\mathrm{ev}_{0}\right)^{-1}: \mathrm{KK}\left(\mathrm{C}(M), \mathrm{C}_{0}\left(T^{*} M\right)\right) \rightarrow \mathrm{KK}(\mathrm{C}(M), \mathbb{C}) .
$$


The arguments in Section 11.2 involving the adiabatic groupoid can be adapted to show that there is a Connes-Thom isomorphism $\Psi$ such that the following diagram commutes

$$
\begin{aligned}
& \mathrm{KK}\left(\mathrm{C}(M), \mathrm{C}_{0}\left(T^{*} M\right)\right) \stackrel{\Psi}{\longrightarrow} \mathrm{KK}\left(\mathrm{C}(M), \mathrm{C}^{*}\left(T_{H} M\right)\right) \\
& \downarrow \mathrm{ev}_{1} \circ\left(\mathrm{ev}_{0}\right)^{-1} \stackrel{\mathrm{ev}_{1}^{H} \circ\left(\mathrm{ev}_{0}^{H}\right)^{-1}}{\longleftarrow} \\
& \mathrm{KK}(\mathrm{C}(M), \mathbb{C}) \text {. }
\end{aligned}
$$

As in $\mathbf{B v E 1 4}$, one can use the natural transformation $\alpha_{M}$ that maps $\operatorname{KK}(\mathbb{C}, A)$ to $\mathrm{KK}(\mathrm{C}(M), A)$ for $\mathrm{C}(M)$-algebras $A$. It makes

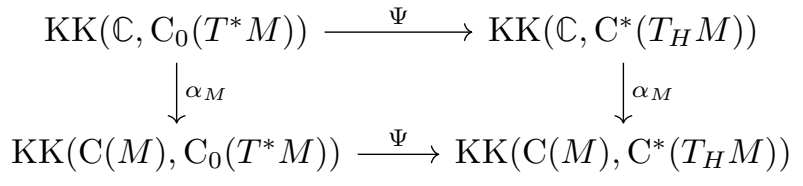

commute. Since the principal cosymbol of $P$ was assumed to be unitary, we can take $\mathbb{Q}_{0}=\mathbb{P}_{0}^{*}$ to construct $\sigma_{H}(P) \in \mathrm{K}_{0}\left(\mathrm{C}^{*}\left(T_{H} M\right)\right)$. This class can be represented in $\mathrm{KK}\left(\mathbb{C}, \mathrm{C}^{*}\left(T_{H} M\right)\right)$ using the Fredholm module given by

$$
\begin{aligned}
\mathcal{E}_{0} & =\Gamma_{0}(E) \otimes_{\mathrm{C}(M)} \mathrm{C}^{*}\left(T_{H} M\right) \oplus \Gamma_{0}(F) \otimes_{\mathrm{C}(M)} \mathrm{C}^{*}\left(T_{H} M\right), \\
\mathbb{D}_{0} & =\left(\begin{array}{cc}
0 & \mathbb{P}_{0}^{*} \\
\mathbb{P}_{0} & 0
\end{array}\right) .
\end{aligned}
$$

Its class is mapped to $\left[\mathbb{D}_{0}\right]$ by $\alpha_{M}$. Together with the commutativity of $(55)$, this shows that [ind $P]=\mathrm{ev}_{1} \circ\left(\mathrm{ev}_{0}\right)^{-1} \circ \alpha_{M} \circ \Psi^{-1}\left(\sigma_{H}(P)\right)$. Therefore, the claim is reduced to the Atiyah-Singer Index Theorem. 


\section{CHAPTER 12}

\section{Conclusion and outlook}

In this thesis, we showed that the generalized fixed point algebra construction can be applied to the zoom action on the tangent groupoid of a filtered manifold. It recovers the order zero pseudodifferential extension of known calculi in this context. Many properties of the calculus follow from the generalized fixed point algebra construction in a natural way. Moreover, it allows to compute the K-theory of the algebra of principal cosymbols and to proceed towards index theory.

In the following, we sketch some related questions and situations to which this approach could be applied as well.

The generalized fixed point algebra corresponds to the operators of order zero. This leads to the question what can be said about operators of different order. It is a result of [DS14], that every classical pseudodifferential operator of order $m$ on a manifold $M$ can be written as an average

$$
\int_{0}^{\infty} \lambda^{m} \sigma_{\lambda}(f) \frac{\mathrm{d} \lambda}{\lambda} \quad \text { with } f \in \mathcal{R} \text { and } f_{0} \in \mathcal{S}_{0}(T M)
$$

It was not shown yet that this could also be done in the filtered manifold case. Understanding this in a systematic way would be also important for cases where no pseudodifferential calculus is available yet and one wants to define it via a generalized fixed point algebra construction.

The Shubin calculus on $\mathbb{R}^{n}$ (see $[\mathbf{S h u 0 1}]$ ), can be understood using an $\mathbb{R}_{>0^{-}}$ action on the tangent groupoid of $\mathbb{R}^{n}$. It is defined for $\lambda>0$ by

$$
\begin{aligned}
\lambda \cdot(x, X, 0) & =\left(\lambda x, \lambda^{-1} X, 0\right) & & \text { for } X \in T_{x} \mathbb{R}^{n} \\
\lambda \cdot(x, y, t) & =\left(\lambda x, \lambda y, \lambda^{2} t\right) & & \text { for } x, y \in \mathbb{R}^{n} \text { and } t>0 .
\end{aligned}
$$

The induced action on $\mathrm{C}^{*}\left(T \mathbb{R}^{n}\right)$ corresponds under Fourier transform to the scalings $\lambda \cdot(x, \xi)=(\lambda x, \lambda \xi)$ on $T^{*} \mathbb{R}^{n}$. The generalized fixed point algebra of an ideal $\sqrt{ } \triangleleft \mathrm{C}^{*}\left(\mathbb{T} \mathbb{R}^{n}\right)$ yields a sequence

$$
\mathbb{K} \longleftrightarrow \operatorname{Fix}^{\mathbb{R}>0}(\mathbb{J}) \longrightarrow \mathrm{C}\left(S^{2 n-1}\right) .
$$

It is the $\mathrm{C}^{*}$-completion of the order zero extension of the Shubin calculus. As a natural continuation, one can replace $\mathbb{R}^{n}$ by a graded Lie group and the scalings by the dilations. In the case where $\mathbb{R}^{n}$ is equipped with different dilations, this should be compared to the anisotropic Shubin classes defined in BN03. For $\mathbb{R}^{n}$ there is an index theorem by Callias (see Cal78, BS78]). For non-Abelian groups one could study the index theory of the resulting operators. In particular, it should be possible to compute the K-theory of the resulting symbol algebra.

All groupoids were equipped with $\mathbb{R}_{>0}$-actions so far. To expand this, one could consider multiparameter actions of $\mathbb{R}_{>0}^{k}$ on $\mathbb{R}^{n}$ of the form

$$
\lambda \cdot x=\left(\lambda_{1}^{a_{11}} \cdots \lambda_{k}^{a_{1 k}} x_{1}, \ldots, \lambda_{1}^{a_{n 1}} \cdots \lambda_{k}^{a_{n k}} x_{n}\right) \text { for } \lambda \in \mathbb{R}^{k} \text { and } x \in \mathbb{R}^{n},
$$

for a fixed matrix $\left(a_{i j}\right)$ with certain properties. Ricci and Stein considered in RS92 convolution operators with kernels that are homogeneous for these dilations. It would be interesting to understand if these are generalized fixed points of the 
action above. If this is possible, one can consider corresponding variable coefficient operators as in NRSW18 and try to relate them to a generalized fixed point algebra attached to an appropriate tangent groupoid. 


\section{Bibliography}

[ADR00] Claire Anantharaman-Delaroche and Jean Renault, Amenable groupoids, Monographies de L'Enseignement Mathématique [Monographs of L'Enseignement Mathématique], vol. 36, L'Enseignement Mathématique, Geneva, 2000. With a foreword by Georges Skandalis and Appendix B by E. Germain. MR1799683

[AS68] Michael F. Atiyah and Isadore M. Singer, The index of elliptic operators. I, Ann. of Math. (2) 87 (1968), 484-530, DOI 10.2307/1970715. MR236950

[Atk51] Frederick V. Atkinson, The normal solubility of linear equations in normed spaces, Mat. Sbornik N.S. 28(70) (1951), 3-14. MR0042059

[BvE14] Paul F. Baum and Erik van Erp, K-homology and index theory on contact manifolds, Acta Math. 213 (2014), no. 1, 1-48, DOI 10.1007/s11511-014-0114-5. MR3261009

[BG88] Richard Beals and Peter Greiner, Calculus on Heisenberg manifolds, Annals of Mathematics Studies, vol. 119, Princeton University Press, Princeton, NJ, 1988. MR953082

[BBL16] Ingrid Beltiţă, Daniel Beltiţă, and Jean Ludwig, Fourier transforms of $C^{*}$-algebras of nilpotent Lie groups, International Mathematics Research Notices 2017 (2016), no. 3, 677-714.

[BN03] Paolo Boggiatto and Fabio Nicola, Non-commutative residues for anisotropic pseudodifferential operators in $\mathbb{R}^{n}$, J. Funct. Anal. 203 (2003), no. 2, 305-320, DOI 10.1016/S0022-1236(03)00194-0. MR2003350

[BS78] Raoul Bott and Robert Seeley, Some remarks on the paper of Callias: "Axial anomalies and index theorems on open spaces" [Comm. Math. Phys. 62 (1978), no. 3, 213-234; MR 80h:58045a], Comm. Math. Phys. 62 (1978), no. 3, 235-245. MR507781

[Bro73] Ian D. Brown, Dual topology of a nilpotent Lie group., Ann. Sci. Éc. Norm. Supér. (4) 6 (1973), 407-411.

[Cal78] Constantine Callias, Axial anomalies and index theorems on open spaces, Comm. Math. Phys. 62 (1978), no. 3, 213-234. MR507780

[Cas16] Bill Casselman, Essays on representations of real groups. The theorem of Dixmier E5 Malliavin (2016), available at https://www.math.ubc.ca/ cass/research/pdf/ Dixmier-Malliavin.pdf

[CP17] Woocheol Choi and Raphaël Ponge, Privileged Coordinates and Nilpotent Approximation of Carnot Manifolds, I. General Results, Journal of Dynamical and Control Systems (2017), 1-49.

[CP19a] Woocheol Choi and Raphaël Ponge, Privileged coordinates and nilpotent approximation for Carnot manifolds, II. Carnot coordinates, J. Dyn. Control Syst. 25 (2019), no. 4, 631-670, DOI 10.1007/s10883-019-09434-9. MR3995957

[CP19b] Woocheol Choi and Raphaël Ponge, Tangent maps and tangent groupoid for Carnot manifolds, Differential Geometry and its Applications 62 (2019), 136-183.

[Chr88] Michael Christ, On the regularity of inverses of singular integral operators, Duke Math. J. 57 (1988), no. 2, 459-484, DOI 10.1215/S0012-7094-88-05721-3. MR962516

[CGGP92] Michael Christ, Daryl Geller, Paweł Głowacki, and Larry Polin, Pseudodifferential operators on groups with dilations, Duke Math. J. 68 (1992), no. 1, 31-65, DOI 10.1215/S0012-7094-92-06802-5. MR1185817

[Con81] Alain Connes, An analogue of the Thom isomorphism for crossed products of a $C^{*}$ algebra by an action of $\mathbf{R}$, Adv. in Math. 39 (1981), no. 1, 31-55, DOI 10.1016/00018708(81)90056-6. MR605351

[Con94] _ Noncommutative geometry, Academic Press, Inc., San Diego, CA, 1994. MR1303779

[CG90] Lawrence J. Corwin and Frederick P. Greenleaf, Representations of nilpotent Lie groups and their applications, Cambridge Studies in Advanced Mathematics, Cambridge University Press, 1990.

[CMR07] Joachim Cuntz, Ralf Meyer, and Jonathan M. Rosenberg, Topological and bivariant $K$-theory, Oberwolfach Seminars, vol. 36, Birkhäuser Verlag, Basel, 2007. MR2340673 
[DH17] Shantanu Dave and Stefan Haller, Graded hypoellipticity of BGG sequences, 2017. preprint available at https://arxiv.org/abs/1705.01659

[DL10] Claire Debord and Jean-Marie Lescure, Index theory and groupoids, Geometric and topological methods for quantum field theory, Cambridge Univ. Press, Cambridge, 2010, pp. 86-158, DOI 10.1017/CBO9780511712135.004. MR2648649

[DS14] Claire Debord and Georges Skandalis, Adiabatic groupoid, crossed product by $\mathbb{R}_{+}^{*}$ and pseudodifferential calculus, Adv. Math. 257 (2014), 66-91, DOI 10.1016/j.aim.2014.02.012. MR3187645

[Dix59] Jacques Dixmier, Sur Les Representations Unitaires Des Groupes de Lie Nilpotents. I, American Journal of Mathematics 81 (1959), no. 1, 160-170.

[Dix77] , $C^{*}$-algebras. Transl. from the French by Francis Jellett., North-Holland Mathematical Library, vol. 15, North-Holland, Amsterdam, 1977.

[DM78] Jacques Dixmier and Paul Malliavin, Factorisations de fonctions et de vecteurs indéfiniment différentiables, Bull. Sci. Math. (2) 102 (1978), no. 4, 307-330 (French, with English summary).

[Dye70] Joan L. Dyer, A nilpotent Lie algebra with nilpotent automorphism group, Bull. Amer. Math. Soc. 76 (1970), 52-56, DOI 10.1090/S0002-9904-1970-12364-3. MR0249544

[Eps99] Charles Epstein, Lectures on indices and relative indices on contact and CR-manifolds (1999), available at https://www.math.upenn.edu/ cle/papers/luminy.pdf.

[vE10a] Erik van Erp, The Atiyah-Singer index formula for subelliptic operators on contact manifolds. Part I, Ann. of Math. (2) 171 (2010), no. 3, 1647-1681, DOI 10.4007/annals.2010.171.1647. MR2680395

[vE10b] . The Atiyah-Singer index formula for subelliptic operators on contact manifolds. Part II, Ann. of Math. (2) 171 (2010), no. 3, 1683-1706, DOI 10.4007/annals.2010.171.1683. MR2680396

[vEY19] Erik van Erp and Robert Yuncken, A groupoid approach to pseudodifferential calculi, J. Reine Angew. Math. 756 (2019), 151-182, DOI 10.1515/crelle-2017-0035. MR4026451

[vEY17] _ On the tangent groupoid of a filtered manifold, Bulletin of the London Mathematical Society 49 (2017), no. 6, 1000-1012.

[Ewe20] Eske E. Ewert, Pseudo-differential extension for graded nilpotent Lie groups, 2020. preprint available at https://arxiv.org/abs/2002.01875

[FR16] Véronique Fischer and Michael Ruzhansky, Quantization on nilpotent Lie groups, Progress in Mathematics, vol. 314, Birkhäuser/Springer, 2016.

[FFK17] Véronique Fischer and Clotilde Fermanian-Kammerer, Defect measures on graded lie groups, 2017. preprint available at https://arxiv.org/abs/1707.04002

[Fol75] Gerald B. Folland, Subelliptic estimates and function spaces on nilpotent Lie groups, Ark. Mat. 13 (1975), no. 2, 161-207, DOI 10.1007/BF02386204. MR494315

[Fol77] _ Applications of analysis on nilpotent groups to partial differential equations, Bulletin of the American Mathematical Society 83 (1977), no. 5, 912-930.

[FS74] Gerald B. Folland and Elias M. Stein, Estimates for the $\bar{\partial}_{b}$ complex and analysis on the heisenberg group, Communications on Pure and Applied Mathematics 27 (1974), no. 4, 429-522, DOI 10.1002/cpa.3160270403.

[FS82] _ Hardy spaces on homogeneous groups, Mathematical Notes, vol. 28, Princeton University Press, Princeton, N.J.; University of Tokyo Press, Tokyo, 1982.

[Gel83] Daryl Geller, Liouville's theorem for homogeneous groups, Comm. Partial Differential Equations 8 (1983), no. 15, 1665-1677, DOI 10.1080/03605308308820319. MR729197

[Gło91] Paweł Głowacki, The Rockland condition for nondifferential convolution operators. II, Studia Math. 98 (1991), no. 2, 99-114, DOI 10.4064/sm-98-2-99-114. MR1100916

[GR79] Elliot C. Gootman and Jonathan Rosenberg, The structure of crossed product $C^{*}$ algebras: a proof of the generalized Effros-Hahn conjecture, Invent. Math. 52 (1979), no. 3, 283-298, DOI 10.1007/BF01389885. MR537063

[Gre77] Philip Green, $C^{*}$-algebras of transformation groups with smooth orbit space., Pac. J. Math. 72 (1977), 71-97.

[HN79] Bernard Helffer and Jean F. Nourrigat, Caracterisation des opérateurs hypoelliptiques homogènes invariants à gauche sur un groupe de Lie nilpotent gradué, Comm. Partial Differential Equations 4 (1979), no. 8, 899-958, DOI 10.1080/03605307908820115 (French). MR537467

[HS87] Michel Hilsum and Georges Skandalis, Morphismes K-orientés d'espaces de feuilles et fonctorialité en théorie de Kasparov (d'après une conjecture d'A. Connes), Ann. Sci. École Norm. Sup. (4) 20 (1987), no. 3, 325-390 (French, with English summary). MR925720 
[aHRW00] Astrid an Huef, Iain Raeburn, and Dana P. Williams, An equivariant Brauer semigroup and the symmetric imprimitivity theorem, Trans. Amer. Math. Soc. 352 (2000), no. 10, 4759-4787, DOI 10.1090/S0002-9947-00-02618-0.

[aHRW02] Astrid an Huef, Iain Raeburn, and Dana Williams, A symmetric imprimitivity theorem for commuting proper actions, Canadian Journal of Mathematics 57 (2002), DOI 10.4153/CJM-2005-038-2.

[Kas88] Gennadi G. Kasparov, Equivariant KK-theory and the Novikov conjecture, Invent. Math. 91 (1988), no. 1, 147-201, DOI 10.1007/BF01404917. MR918241

[KW99] Eberhard Kirchberg and Simon Wassermann, Exact groups and continuous bundles of $C^{*}$-algebras, Math. Ann. 315 (1999), no. 2, 169-203, DOI 10.1007/s002080050364. MR1721796

[Kir62] Alexandre A. Kirillov, Unitary representations of nilpotent Lie groups., Russ. Math. Surv. 17 (1962), no. 4, 53-104 (English).

[KS71] Anthony W. Knapp and Elias M. Stein, Intertwining operators for semisimple groups, Ann. of Math. (2) 93 (1971), 489-578, DOI 10.2307/1970887. MR460543

[KM20] Bartosz Kosma Kwaśniewski and Ralf Meyer, Stone duality and quasi-orbit spaces for generalised $C^{*}$-inclusions, Proc. Lond. Math. Soc. (3) 121 (2020), no. 4, 788-827, DOI 10.1112/plms.12332. MR4105787

[LR01] Nicolaas P. Landsman and Birant Ramazan, Quantization of Poisson algebras associated to Lie algebroids, Groupoids in analysis, geometry, and physics (Boulder, CO, 1999), Contemp. Math., vol. 282, Amer. Math. Soc., Providence, RI, 2001, pp. 159192, DOI 10.1090/conm/282/04685. MR1855249

[Lee76] Ru Ying Lee, On the $C^{*}$-algebras of operator fields, Indiana Univ. Math. J. 25 (1976), no. 4, 303-314, DOI 10.1512/iumj.1976.25.25026. MR410400

[LMV17] Jean-Marie Lescure, Dominique Manchon, and Stéphane Vassout, About the convolution of distributions on groupoids, J. Noncommut. Geom. 11 (2017), no. 2, 757-789, DOI 10.4171/JNCG/11-2-10. MR3669118

[Mac05] Kirill C. H. Mackenzie, General theory of Lie groupoids and Lie algebroids, London Mathematical Society Lecture Note Series, vol. 213, Cambridge University Press, Cambridge, 2005. MR2157566

[Mel82] Anders Melin, Lie filtrations and pseudo-differential operators, 1982. unpublished.

[Mey01] Ralf Meyer, Generalized fixed point algebras and square-integrable group actions, J. Funct. Anal. 186 (2001), 167-195, DOI 10.1006/jfan.2001.3795.

[Mil17] Philip Miller, The generalized fixed point algebra for the scaling action on the tangent groupoid, Master's Thesis, 2017, https://www.uni-math.gwdg.de/rameyer/website/ Miller_Master.pdf

[Moh18] Omar Mohsen, On the deformation groupoid of the inhomogeneous pseudo-differential Calculus, 2018. preprint available at https://arxiv.org/abs/1806.08585

[Moh20] _ Index theorem for inhomogeneous hypoelliptic differential operators, 2020. preprint available at https://arxiv.org/abs/2001.00488

[NRSW18] Alexander Nagel, Fulvio Ricci, Elias M. Stein, and Stephen Wainger, Algebras of singular integral operators with kernels controlled by multiple norms, Mem. Amer. Math. Soc. 256 (2018), no. 1230, vii+141, DOI 10.1090/memo/1230. MR3862599

[Nil96] May Nilsen, $C^{*}$-bundles and $C_{0}(X)$-algebras, Indiana University Mathematics Journal (1996), 463-477.

[Nis03] Victor Nistor, An index theorem for gauge-invariant families: the case of solvable groups, Acta Math. Hungar. 99 (2003), no. 1-2, 155-183, DOI 10.1023/A:1024517714643. MR1973092

[Pat99] Alan L. T. Paterson, Groupoids, inverse semigroups, and their operator algebras, Progress in Mathematics, vol. 170, Birkhäuser Boston, Inc., Boston, MA, 1999. MR1724106

[Ped89] Niels V. Pedersen, Geometric quantization and the universal enveloping algebra of a nilpotent Lie group, Transactions of the American Mathematical Society 315 (1989), no. 2, 511-563.

[Pon08] Raphaël S. Ponge, Heisenberg calculus and spectral theory of hypoelliptic operators on Heisenberg manifolds, Mem. Amer. Math. Soc. 194 (2008), no. 906, viii+ 134, DOI 10.1090/memo/0906. MR2417549

[Puk67] Lajos Pukánszky, Leçons sur les représentations des groupes, Monographies de la Société Mathématique de France, No. 2, Dunod, Paris, 1967 (French). MR0217220

[Puk71] _ Unitary representations of solvable Lie groups, Ann. Sci. École Norm. Sup.(4) 4 (1971), 457-608 
[RW98] Iain Raeburn and Dana P. Williams, Morita equivalence and continuous-trace $C^{*}$ algebras, Mathematical Surveys and Monographs, vol. 60, American Mathematical Society, Providence, RI, 1998. MR1634408

[RW85] , Pull-backs of $C^{*}$-algebras and crossed products by certain diagonal actions, Trans. Amer. Math. Soc. 287 (1985), no. 2, 755-777, DOI 10.2307/1999675.

[Ren80] Jean Renault, A groupoid approach to $C^{*}$-algebras, Lecture Notes in Mathematics, vol. 793, Springer, Berlin, 1980. MR584266

[RS92] Fulvio Ricci and Elias M. Stein, Multiparameter singular integrals and maximal functions, Ann. Inst. Fourier (Grenoble) 42 (1992), no. 3, 637-670 (English, with English and French summaries). MR1182643

[Rie82] Marc A. Rieffel, Applications of strong Morita equivalence to transformation group $C^{*}$-algebras, Operator algebras and applications, Part I (Kingston, Ont., 1980), Proc. Sympos. Pure Math., vol. 38, Amer. Math. Soc., Providence, R.I., 1982, pp. 299-310. MR679709

[Rie90] - Proper actions of groups on $C^{*}$-algebras, Mappings of operator algebras (Philadelphia, PA, 1988), Progr. Math., vol. 84, Birkhäuser Boston, Boston, MA, 1990, pp. 141-182. MR1103376

[Rie04] - Integrable and proper actions on $C^{*}$-algebras, and square-integrable representations of groups, Expositiones Math. 22 (2004).

[RS76] Linda Preiss Rothschild and Elias M. Stein, Hypoelliptic differential operators and nilpotent groups, Acta Math. 137 (1976), no. 3-4, 247-320, DOI 10.1007/BF02392419. MR436223

[CR08] Paulo Carrillo Rouse, A Schwartz type algebra for the tangent groupoid, K-theory and noncommutative geometry, 2008, pp. 181-199, DOI 10.4171/060-1/7.

[Rud91] Walter Rudin, Functional analysis, 2nd ed., International Series in Pure and Applied Mathematics, McGraw-Hill, Inc., New York, 1991. MR1157815

[SH18] Ahmad R. H. S. Sadegh and Nigel Higson, 10. Euler-Like Vector Fields, Deformation Spaces and Manifolds with Filtered Structure, DOCUMENTA MATHEMATICA 23 (2018), 293-325.

[Shu01] Mikhail A. Shubin, Pseudodifferential operators and spectral theory, 2nd ed., SpringerVerlag, Berlin, 2001. Translated from the 1978 Russian original by Stig I. Andersson. MR1852334

[Tan70] Noboru Tanaka, On differential systems, graded Lie algebras and pseudogroups, J. Math. Kyoto Univ. 10 (1970), 1-82, DOI 10.1215/kjm/1250523814. MR266258

[Tay84] Michael E. Taylor, Noncommutative microlocal analysis. I, Mem. Amer. Math. Soc. 52 (1984), no. 313, iv+182, DOI 10.1090/memo/0313. MR764508 